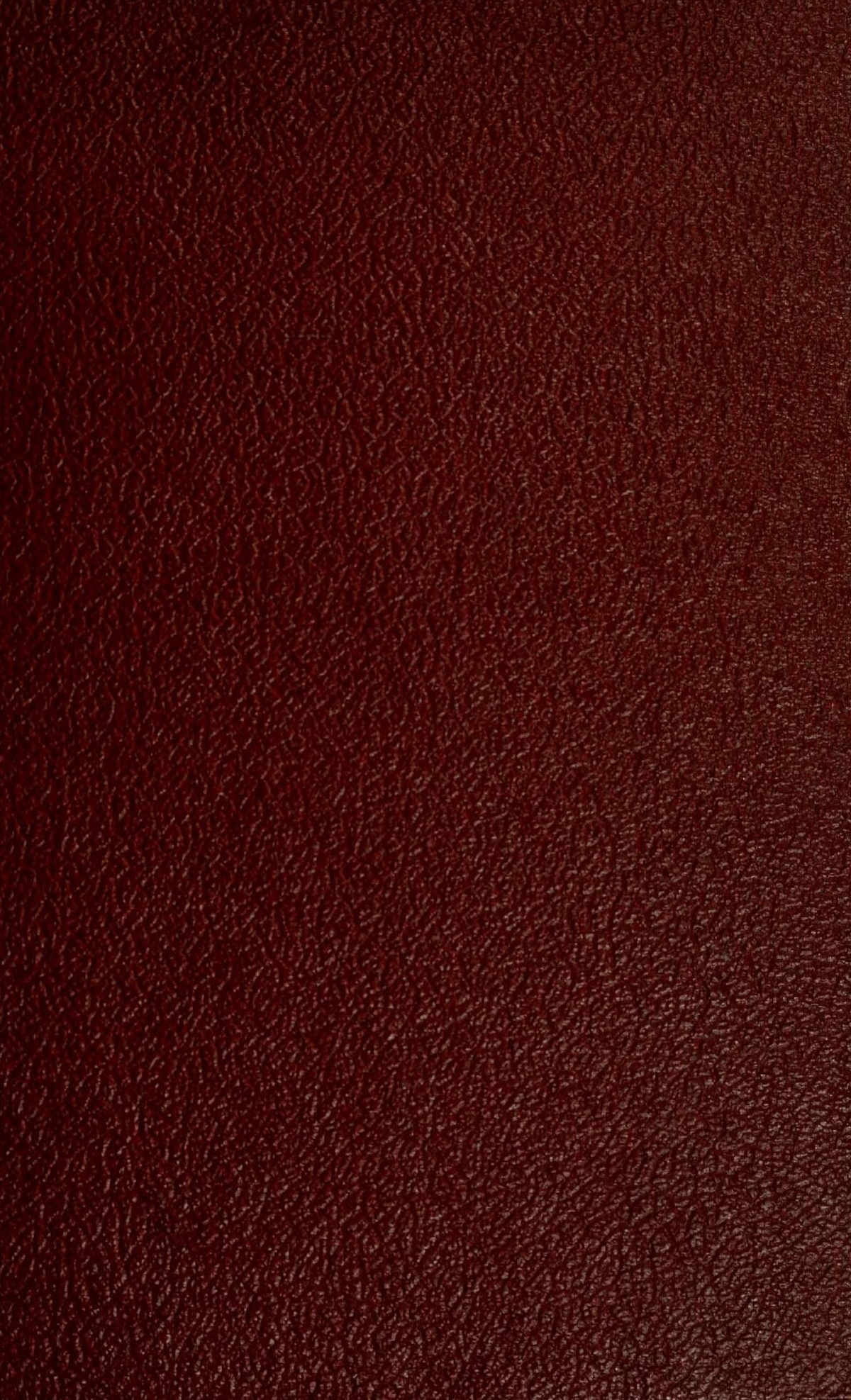



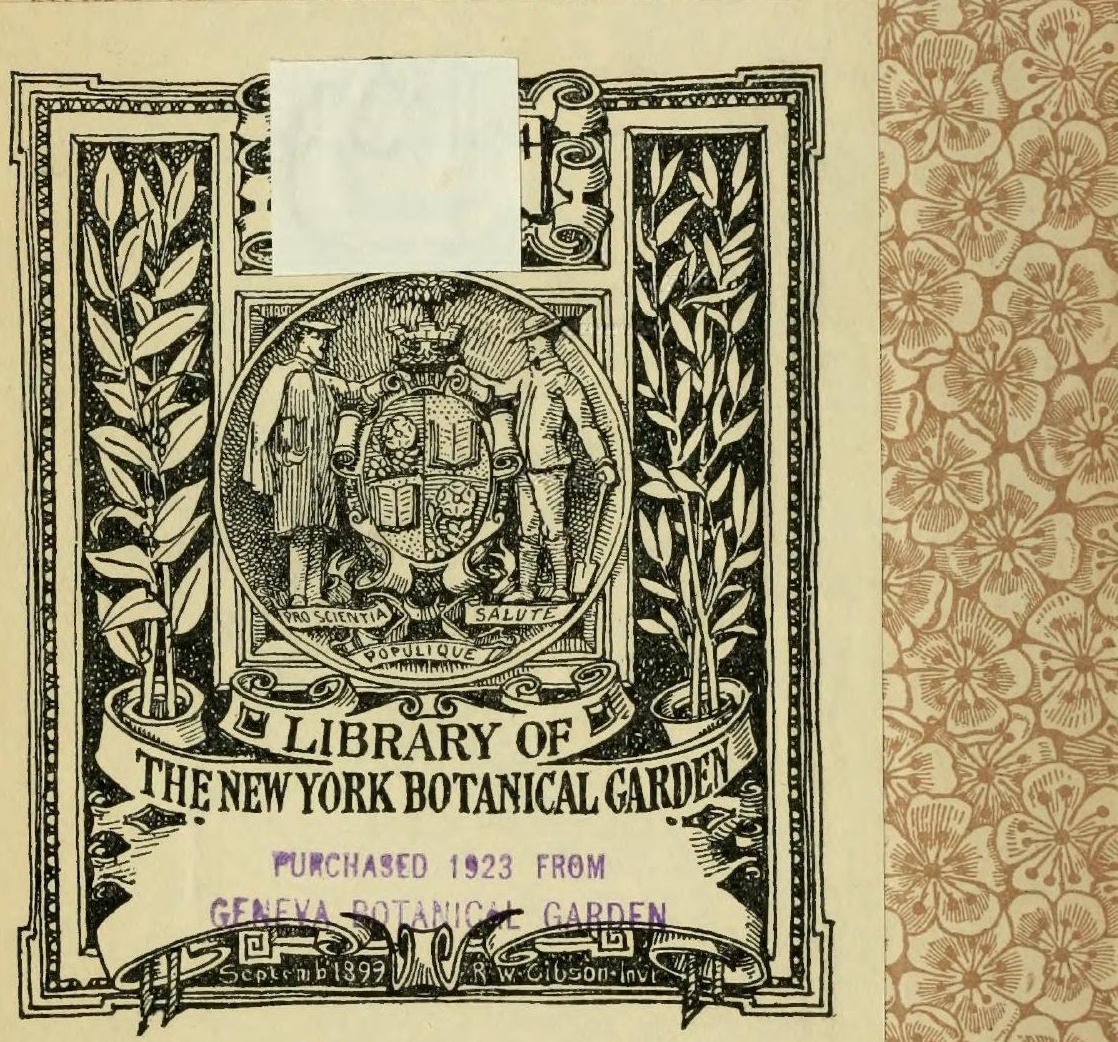







\section{HERBORISATIONS}

A

SA L E VE

PAR

Ie docteur Ch. FAUCONNET

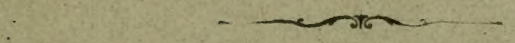

GENEVE

IMPRIMERIE CABEY FRÈRES, VIEUX-COLLÉGE, ;

1867 
Plempicen le yrerpary

Perrwiquage be

\section{HERBORISTTIONS}

\section{SALEVE}

III:

le doctem Ch. Raúconivét

$\because 4 T H$ WH

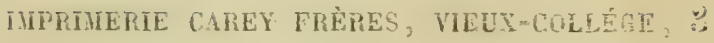

1867 



\section{DÉDICACE}

\section{Aux anciens Mrembres de la Socińtri Mallérieme de Bolani(y)e}

Chers, dignes et respectables amis!

Bien que la Société que nous avions fondée en commun soit tombée en sommeil, notre amitié ne s'est pourtant point endormie; c'est ce qui mencourage it placer sous votre pulronage ces quelques payes, qui devaient paraitre dans les Bulletins de la Sociéti Hallérienne que j’ai rédigés jadis d'après les procés-verbunx de nos séances el les documents que vous m’aviez fournis.

Cet opuscule sera un souvenir de nos réunions passées et de nos herborisations à Saleve. Il vous rappellera peut-itre quelques heureux moments, tout en vous apportant un souvenir affectueux de l'ex-président de feu la Société Hallérienne de Botanique.

Genève, Mars 1867. 



\section{PRÉLUDE}

Le mont Silève n'est curtes pas beau! Sa croup lase el chauve, ses flanes abruples el dénudés ne précentent rien de pittoresque ni de gracieux. A voir sil masse informe et lourde, on dirait quelque monstrueux ruptile des époques qui ont précédé la création acluelle. Eh bien! malgré cet aspect peu attrayant, il est aimé des Genevois; c'est leur montagne par excellence, leur promenade favorite, et sa vue réveille toujours en cux de brillants souvenirs de jeunesse et la glorieuse mémoire des premières excursions. Le père qui conduii ses enfants sur un des points culminants de la monta- 
gne, s'associe a leu joie ul relrouve les impressions yu’il éprouva jadis, la premièce fois qu'il alteignit ce sommet lant convoilé. P'uis on aime à se souvenir des parlies faites avec les camarades de Collége, quand on marchait pour obéir au besoin de locomotion, qu'on grimpait pour le plaisir de monter et qu'on élail ficr d'avoit fait le plus prand tour possible, dans un jour de congé.

Puis encore, quelle jouissance n'éprouve-t-on pas it se rappeler les souvenirs des premières excur'sions scientifiques de l'étudiant, alor's que partant soil avec la capsule et le cartable du botaniste, soit avec le marleau du géologue, soit avec le filet de l'entomologiste, chacun suivant son goût, récoltait les plantes, dégageail les fossiles on chassait les coléoptères et les patpillons.

lien souvent, en retrouvant dans mon herbier des echantillons séchés depuis une quaranlaine d'années, j’ai rovu la sympathique figure du professeur Seringe quii nous dirigeait dans nos premières herborisalions.

Quel bonheur surtoul d'être ell société de hons r't d'aimables compagnons sachant se faire les uns aux aulles. Que de conversations animées et intéressantes, que de discussions à bàtons rompus, que de bons: rires faisant oublier les années et les soucis! (Uuand vient une halte destinće à se reposer el à sojünceles récoltes, on ne reste pas insensible aux charmes de l'omelelte el de lis silade, à l'attrait d'un verre de bière mousseuse ou du pelit blane souvent acidule, mais loujours rafraichissant.

Bien que rivaux comrtisant l'aimable Flore, leur gritbieuse souveraine, les botanistes vivent généralement en paix les uns avec les autres. Il est rare que la gucre s'allume entre eux; ils sont sur un terrain new- 
tre sur lequel les opinions les plus opposées peuven t se coudoyer sans se heurter brusquement, et la poursuite innocente qu'ils font en commun impose silence, aux trop nombreuses causes de querelles qui divisent l'espèce humaine. A vivre avec les simples, on devient meilleur et plus tolérant.

Voyez que de douces jouissances accompagnent l'étude de la botanique et les herborisations qui en sont le complément indispeusable. Pas de répugnance comme dans les dissections et les recherches des zoologistes; si l'on coupe un rameau, on ne voit pas dégoutter du sang; si l'on met en presse une plante, on n'a pas à s'endurcir contre les convulsions de l'agonic et les cris de la douleur. La gentiane du printemps, dans son linceuil de papier, vous suit avec son wil bleu, d'un regard triste et résigné, mais sans expression de souffrance et de reproche.

En outre, quel champ d'études offert aux naturalistes par les rapprochements intéressants que la botanique rappelle au moyen de ses rapports avec l'élat physique et la composition du sol, avec l'altitude, l'exposition, le degré de sécheresse ou d'humidité et toutes les circonstances variées qui rendent compte de la dispersion et des stations des végétaux; que de considérations curieuses suggère l'examen de leur:r'apports avec les insectes dont on est toujours assuré de trouver certaines espèces là oủ croissent les planles qu'ils affectionnent, observation qui se trouve confirmée par l'élude des plantes et des insecles fossiles, ainsi qu'il ressort des travaux remarquables du professeur 0. Heer de Zurich.

Enfin, que de choses merveilleuses à découvrir dans les habitudes, les instincts et la vie de ces fleurs obligées à tant de luttes et de sagacité pour vivre d'abort puis pour propager leur lignée. 
A mesure que les années s'écuulent, les forces dimimuent, la fatigue se fait plus vite sentir, et le besoin le repos tinil patr domimer; an ast lested de remplater

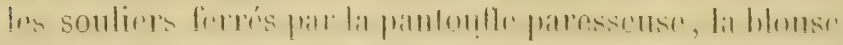
par la robe de chambre, la sigge de lochers moussus par le fauteuil rembomré. C'est alors ou jamais quil

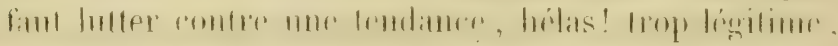
mais qui finil pat énerver et vieillit avant le temps.

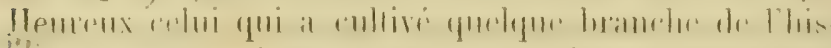
lowe nalurelle, il trouvera lit un stimuland propres it

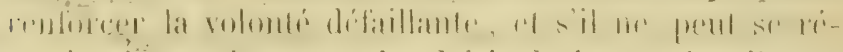
soudre at marcher pour le plaisir de la marche, il aura l'allrait de recherches intéressantes ef d'm exercice

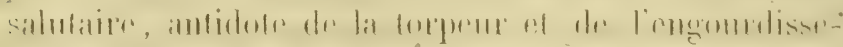
ment. Caest surtout en automme, quand on firil ume

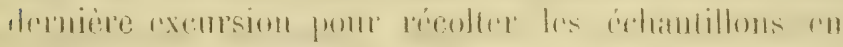

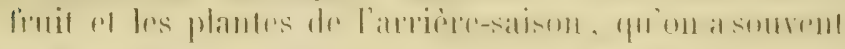

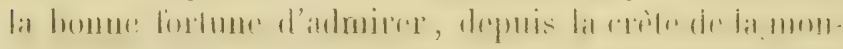

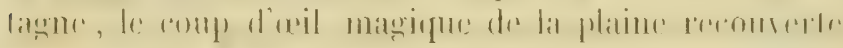

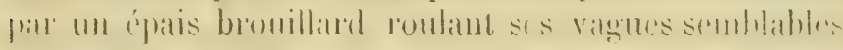
a celles de l'Océan. Celle mer immense et sombre, - remplicant la nappe bleue de notre beau lac, ost li-

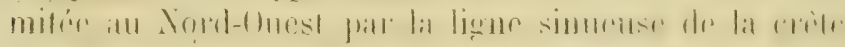
du Jura, ol au sud-kst par la chaine étincelante des

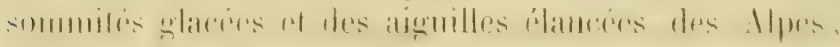
qui s'étendent depuis le lae d'Annecy jusqu'au canton

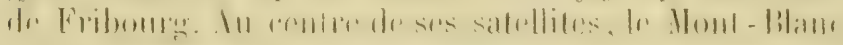
mrine daus son inmuable majesté.

Cependant les insectes bourdonnent, les dernières hems smonessent d'ouvrir leurs corolles allardies. quelques oiseaux, prercant le lugubre linceuil qui recouvere la plaime, saluent d'un cri joyeux le chand soleil que leur inslinel est venu chercher. Mais il lint s'arracher it ce spectacle sans égal of se replonger, en 


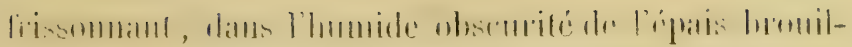
larrl qui eache la vallée à nos yeux.

Dins la même sitison, lorspre le temps est clair, on

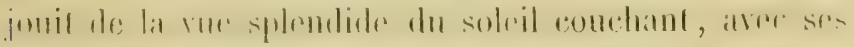
magnificences que la plume ne peut décrire, que le pincean ne pent reprósenter.

On sent alor le coent s’apaiser, l'ime se rassérémer

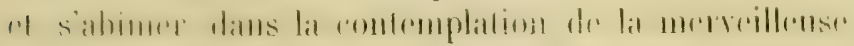
harmonie de l'univers, créé par la Sigesse, soutenu parl la Force etorné par la Beaulé qui viennent d'linHaul. A l'oenvere on leconmait l'Ouvrier, el l'on se pros-

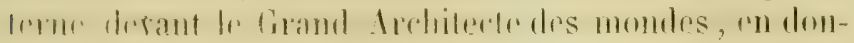
naml oloive a sa somveraine Juslice, i son infunic Miserreorde, at son incomparable saintelé.

Venez done à la monlagne, vous tous qui êles en lulle ave les mecessiles of les soncis to lat vie. Hommes de rabinel ct d'afclier, négociants of savants, vous smlfout, jeunes éfudiants qui devez maintenir los anciennes lradilions bolaniques de Geneve, venez chereher lit des jonissanees incommus a lit ville, venez vous reposer de vos labitudes sedentatres of latigantes, ve110\% respire l'air vil, balsamique el pur des sommeds ilcrés, rous y forez provision de foree, de joire el de allté.

Vicns sumbul, panre midecio, blanchi sous lo hamais, eombe sous te poids de lit responsabilite,

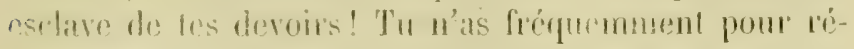
emponse de les soins at de lon dévonement que déhoires el désappointements. Là où lu as donné ton comm, lu es prayé somvent d'jngratilude el de mauvais procédés. Iát où lu n’ás épargné ni ton temps, ni tes fremes, on se déballabse de loi aree quelque chéfive rémunération. Tu ne l'appartiens pas, tu n’as pas le troit de jourir des joies de lat lamille; ton sommeil, 
les lorees et la santé sont la proprićté du public, qui l'a bientôl oublié, lorsque, vicux avant le temps, usé par les faligues, lu ne peux plus répondre à ses exjgenees. Tu devrais èlre seul et remplir la vocation comme un sacerdoce gratuit; autrement tu es à plaindre, si ta profession doit être ton gagne-pain. Cependant II auras toujours pour récompense le contenl'ment intéricur, le sentiment du devoir accompli, l'altection de quelques fidèles clients et de quelques vicux amis. Puis, si tu succombes sous le poids du découragement, tu viendras à la montagne reprendre des forces nouvelles pour la lulle de tous les jours: 


\section{INTRODUCIION}

Le mont Salève est étendu dans la direction du NordEst au Sud-Ouest; sa longueur, depuis Etrembières à son extrémité méridionale, est d'environ 7 lieues. II se divise en quatre parties, nommies : 10 Pelil-Salive: 20 Grand-Salève, entre le vallon de Monnetier el la Croisetie; $3^{0}$ chaine des Pitons, se terminant it la rivière des Usses; $4^{0}$ enfin la Parlic miridionale qui s'élend jusqu'au Fier.

Le sommet du Petit-Salève est à environ 897 mètres aut-dessus du niveau de la mer, Grange-Tournier point culminant du Grand-Salève à 1,286 mètres, le Grand- 
Pitum a 1,383 mètres, Cirtsillw at 785 mètres, le piod du Pas-de-l'Echelle à 614 mètres, Veyrier à 429 el Monnelier à $721{ }^{1}$.

La lace du Salève yui regarde les Alpes présente unc inclinaison douce et uniforme; elle offre des pàturages at quelques bouquels de bois.

La face qui regarde le Jura est, au contraire, coupcir it pic à une assez grande hauteur, el ne présente cju: l's tranches escarpées et nues de ses couches a peu près horizontales et parallèles. Celte face est sillonnér far un grand nombre de gorges, qui en facilitent plus ou moins l'ascension et qui sont riches en bonnes espères. Ces passagres sont : Io sentier des voûtes du Petil. Sateve, le Pas-de-l'Echelle, lo sentier de la Prtileliorge, celui de la Grande-Gorge, la Croisette, Ie sentier des Pilons el celui de Pommier.

La masse de la monlagne est constituée par des couchrs calcaires superposées; l'inféricure, qui est la plu: ipaisse et la plus puissante, appartiont au terrain $\mathrm{Co}_{0}$ iallien; c'est dans cutte couche que sont creusées les 'arrières de Monnelier. Immédiatement an-dessus dı Corallien, s'étend une couche mince? de Porllendien, terrains essentieltoment jurassiques. Enfin, tout à fait it la surlace, existent les conches crétacées du terrain Vryonien et Niocomien, qui se rrtrouvent égalemen! at1 Jura. Sateve fait done partie, an point de vue gíologique, de la chaine du dura.

Le pied des couches abruptes est caché par des lalus d'éboulemenls que le bolaniste parcont loujours avere fruil. Autour de: la base de la montagene, sétendent les courhes molassiques soulevées el ren-

- Voir l'ouvrage de M. le prof. A Favre, intilulé : Considéruliom géoloyigue sur le mont Suleve, 1813. 
verseses, et les terrains diluviens qui constituent la plaine. Silive forme done comme une île calcaire, dont la surface rat recouverte sur plusicurs points de blocs erratiques el de conches sidéroliliques.

Ce dernier terrain se présente lantòt sous forme de roches d'un blane pur, formées par un sable crislallin dont les grains ont peu $\lambda_{1}$ relhésion; lantôt sous forme de couches sablonneusis, qui sont surtout abondantes vers l'extrémité méridionalo de la montagne. Les bloes agrégés se rencontrent au sommet du Grand-Salève, vers la lilande-Gorge. Ces couches, en divers endroits, sont riches en fer hydraté qui a été exploité jadis, el acest probablement à celtr cause qu'il faut attribure lit disparition des forêts do bictres qui combaient andrefois la monlagne ot dont les Treie Arhers affir nl incere dr. nos jours un échantillon.

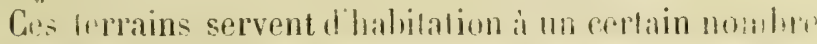
de plantes, qui se plativit dans les sibles silinenx, "1 qui ne se rencontrent pas ailieur's, telle's que : Sclrranthus perennis L., S. biennis Rent., Sedum villosum L., Arnica montana L. Filago minima L., Petlicularis tuberosa L., Betula albu L., Almus rividis DC., Deschampsia flexuosu Ciriscb., Lysoporlium claerutum L., enfin Pteris aquilina L. Je ferai observer cepenlant que cette dernière plante s'élève assez haul sur le Jura et qu'elle ne serail donc pas exchnivement silicicole.

A ce propos, sans nier l'influence que pent exirer sur les stations de cerlaines plantes, la nature chimique du sol (pour les plantes marilimes par exemple), je ne crois pas que l'on puisse piendre pour lase: d'une classification phytostatique la composition rhimique des roches sons-jacentes. Les torrains ne sont jamais absolument purs; lat sol sablonnenx, protuit 
de la désagrégation de roches siliceuses, contiendra toujours une certaine proportion de chanx. - C'est er qui arrive au sable cristallin blanc de Cruseilles. Tel autre sol compacte, produit de la désagrégation de roches calcaires, contiendra toujours une certaine proportion de silice. - C'est ce qui arrive pour la couche calcaire crétacée supérieure de Salève qui fait leu sous le marteau, d'après les observations de M. Bernelt. Il en résulte que les plantes, ne formant pas ces substances de loutes pièces, les choisissent dans le sol en absorbant avec l'eau de végélation les élémerı chimiques qui leur conviennent; seulement les proportions variant suivant les cas, la végétation s'en r'cssentira; anssi, an point de vue de l'agriculture, devra-1-on toujours restituer arlificiellement aux terrains épuisés les éléments absorbés qui finiraient par lui faire défaut.

En résumé, tout en admettant dans certaincs limites l'influence de la composition chimique des roches sousjacentes, j'attribue beaucoup plus d'efficacité à leur degré de dureté ou de friabilité, à la facilité plus on moins grande avec laquelle elles peuvent se désagréger, à leurs propriétés physiques plutòl qu'à leur composition chimique.

En effet, les terrains provenant de la désagrégation de roches dures, - quelle que soil leur nature chimique, - serviront toujours de station à ces végétaux qui aiment un sol compacte, sece chand (plantes dites calcarophiles). En revanche, les terrains provenant de la désagrégation de roches friables, - quelle que soit leur nature chimique, - serviront toujour's de station at ces végétaux qui aiment un sol léger, humide et frais (plantes dites silicicoles) ${ }^{1}$.

1 Voir l'ouvrage de Thurmann : Essai de Phytostatique appliqué à la chaîne du Jura. 
Les blocs erratiques oceupent surtont l'extrémité sepIrntrionale de Salève et la parlie qui regarde les $\Lambda$ lpes; ils sont abondants sur les flancs du Petit-Salève. Ces hlocs proviennent en grande partie des Alpes de la vallée de l'Arve, mais il en est qui viennent de la vallée dı Rhône et des Alpes du Valais; tous ont été transportés par l'ancien glacier qui s'étendait depuis les Alpes jusqa'ai Jura.

Ces blocs sont couverts de certaines espèces de $L i$ chens qui les font reconnaître de loin, el quelques-uns présentent dans leurs fissures des touffes de l'Asplenium septentrionale $\mathbf{L}$. plante qui tend à disparaître par le fait de la destruction de ces blocs qu'elle affictionne exclusivement. Lo. Celerach officinarum CB. se trouve sur les murs construits en pierres cristallines.

La végétation qui repose sur les couches calcaires a hraucoup de rapport avee celle du Jura, aussi troure. t-on un nombre considérable de plantes communes i cros deux montagnes et qui se plaisent dans les mêmes conditions de sol et d'exposition.

Outre les plantes signalées plus haut, il existe un rertain nombre d'espèces spéciales à Salère et qui no se rencontrent pas ailleurs dans les limites de notre Flore. Ce sont :

Atragene alpina L., Fumariu Chavini Roul.. Arabis hybrida Reul., Sisymbrium acutangulum DC., Ononis rotundifolia L. , Rubus collinus DC., Potentilla peticiIntata Gaud., Rosa Sabuuda Rap., R. Salavensis Riap., R. vestite Godet., R. murginutu Wallr., R. coronatu Crep., R. alpestris Rap., Sedum unopetalum DC., Gulium spurium L. y tenerum Gir. ot G., Serratula medicaulis DC., Hieracium pseulo-cerinthe Koch, H. lenatum Vill., $H$. andryaloides Vill., $H$. melanotrichum Pieut., Pyrola media Sw., Cynoglossum montrnum L., 


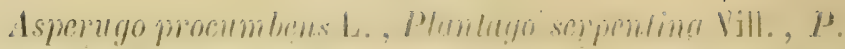

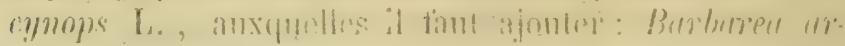

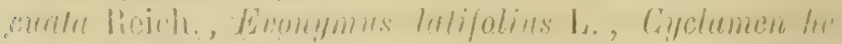

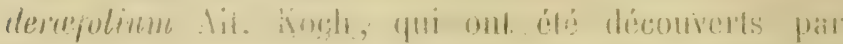
.l. Labhé. Delavay, sur la partie méridionales de la montagne, prie d'Allonzier.

Les plantes rares equi font l'ornernent da lépulitlion du sabeve avaient atliré déji lattention des anciens bolanistes, melstune I. Manhin, Ray, Malechamp, Cherler, C. Sherard, Cl. lactere, Haller, de Sans:sure, Gandin, Girod, de Candolle ot d'aulres encore. 'Tous les botanistes qui ont érit sur la flore

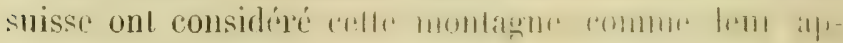
partenant, bien qu'cllo soii en dohors des linters piililiques de nolre pays.

De nos jours, Mll. Limber, hapin, lioissier, Muret. Chavin, Bernell, Jupin, Hichand, Huw, Ducommum, Ph. Prival,- lamnu, Plugel, et d'antres amalenes eneort ont pareonru toules les focalicís de lat monlague, et if serait diltieibr de tromser des plantes qui auraient

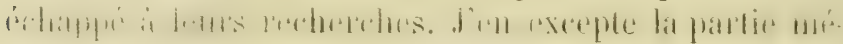
ridionalu, do Cirnseilles au lier, qui a été assez géncralrment négligée el ou l'on pourrait faire des décon pertes intéressantes.

La notice de M. l'abbé Pugel, insérée dans le lorl-

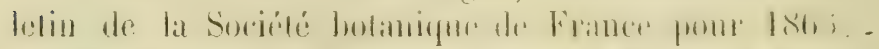

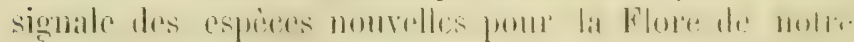
montagne el gui ont élé lrouvées récenment.

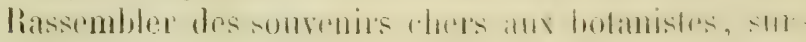

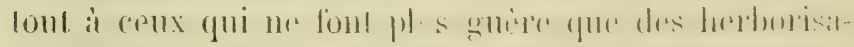

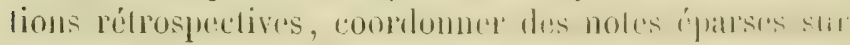

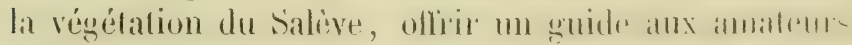

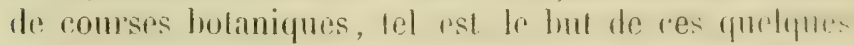
pages qui ne penvent avoir d'auters prétentions. 
PS. - Voir, pour des détails plus circonstanciés sur la structure du Salève, les ouvrages de De Saussure, du professeur A. Favre, du professeur Vogt et le tome XVI des mémoires de la Société d'histoire et d'archéologie de Genève, qui contient un mémoire de M. A. Naville sur les anciennes exploitations de fer du mont Salève, et un mémoire de M. F. Thioly sur les époques antehistoriques de la même montagne. 



\section{IIERBORISATIONS}

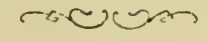

\section{Mois de Mars.}

L'haleine chaude et vivifiante du vent d'Afrique le Foehn de nos monlagnards - a fondu les neiges, verdi les prairies el développé les bourgeons. Déjà les haies se feuillent, et à leur pied on voit s'épanouir les corolles des Corydales, des Primevères, des Scilles et des Violettes.

Essayons une première course à Salève, bien que nous ne soyons qu'au commencement de Mars; nous ne ferons pas une abondante moisson, mais nous serons heureux de revoir les premières fleurs, messagères du printemps, et de faire une promenade après l'inaction forcée de l'hiver. 
Parcourons d'abord le pied et les pentes du PetitSalève, entre Veyrier et Etrembières. Près de la Fontaine, dite de Jules-César, dans les taillis et sous les buissons, se cachent: Hepatica triloba DC., Corydalis tuberosa L., Daphne Mezereum L. avec ses tiges couvertes de fleurs roses parfumées, mais âcres et vẻnéneuses, Daphne Laureola L., Asarum Europaum L., et Scilla bifoliu L., quelquefois à fleurs blanches.

En gravissant les pentes rocailleuses et boisées à gauche du Pas-de-l'Echelle et atl-dessous du chàtcau de Monnetier, on verra sous les taillis Leucoium vernum L., charmante plante printanière qui fleurit souvent en Février, et qu'on retrouve en abondance sur les pelouses situées derrière le vieux chàteau d'Etrembières.

En grimpant depuis cette dernière localité jusqu'au Trou de Tarabara, on récoltera sur la crête du PetitSalève Draba aizoides L., qui couronne presque tous les rochers de la montagne. Enfin, en continuant à remonter vers le sommet, on trouvera parmi les herbes encore desséchées Polygala Chamobuxus L., dont je possède un échantillon en fleurs cueilli le 3 Janvier.

A Mornex, dans les bois, fleurit Viola abortiva Jord., espèce ou variété qui se rapproche du $V$. alba Besser. Entre Mornex et Reignier nous avons trouvé Viola odorata L. var. albiflora, assez rare et qui se reconnaît à ses fleurs odorantes, blanches à éperon violet.

En parcourant, plus tard, les taillis entre Veyrier et le Coin, on récoltera Viola alba Besser., espèce bien caractérisée et qui ne doit pas être confondue avec $V$. odorata var. albiflora. Elle présente deux formes constantes; toutes deux ont des souches lon- 
guement stolonifëres, elles conservent ordinairement jusqu'au printemps les grandes feuilles radicales de l'année précédente, cordiformes, allongées, pointues et couverles de poils à base dilatée; mais l'une a les feuilles ainsi que le calice d'un vert pâle, les corolles blanches avec l'éperon verdàtre, toute la plante a une apparence chlorotique; c'est Viola virescens Jord. Reuter, Rapin. L'autre a les feuilles d'un vert sombre teinté de pourpre, les calices colorés, les corolles blanches avec l'éperon violet, toute la plante a le tempérament sanguin; c'est Viola Scotoplyylla Jord., Reut., Rapin. Toutes deux sont odorantes, mais le parfum en est moins pénétrant que celui de la violette ordinaire; quelquefois on les trouve à fleurs bleues, d'une couleur claire pour la première forme, d'une couleur foncée pour la seconde.

Dans les taillis abondent: Corylus avellanu L., dont les chatons sont déjà tlétris, Salix purpurea L., $S$. cincrea L., S. capraea L., S. aurita L., qu'il faudra reprendre plus tard en feuilles bien développées, $P_{0-}$ pulus tremula L., et $P$. alba L., ce dernier au bas du chemin de la Croisette.

Dans le bois de Veyrier, à gauche, en venant par la route de Pinchat, existe un pied nâle de Salix Pontcderana. Willd. Gaud. Cette forme hybride devra être cherchée plus lard au bas de la Grande-Gorge, les fleurs y sont encore en hon état à la fin d'Avril et les feuilles à la fin de Mai, d'après les indications de M. Rapin.

On cherchera, pour terminer avec les Saulcs, au bord de l'Arve, entre Etrembières et Veyrier, Salix alba L., S. amygdalina L., avec les var., $\alpha$ discolor God. $\beta$ concolor God., S. daphnoides Vill., qui vivent en socićté avec Alnus incana DC. et $A$, glutinosa Gaerın. 
Vers la fin du mois, une promenade ì Reignier et à Pers, par la singulière plaine des rocailles, fera retrouver la plupart des pliutes déjả signalées et d'autres telles que: Hepatica triloba DG., Corydalis bulbosa DC., Polygala Chumabuxus L., Tussilago farfara L., Buxus sempervirens L., Narcissus PscudoNarcissus L., Leucoium vernum L., et Scilla bifoliu L., soit sur les rocher's de la plaine, soit autour du inonument Druidique, connu sous le nom de Picreaux-Fées. A dix minutes de cette dernière localité, en se dirigeant du côté de l'Arve, on tombe sur des taillis remplis d'Erythronium Dens-Canis L. Celle belle Liliacée est abondante dans les bois et ravins de la rive gauche du Rhône, et ne se trouve pas ailleurs en Suisse.

Dans les vergers du village de Pers et des communes avoisinantes, 'du côté de La Roche, on peut faire provision d'Helleborus viridis L., qui n'existe pas en dehors de cette région. Quant à l'Helleborus fatidus L., il est commun dans tous les taillis et les haies du pied de la montagne.

Le Ficaria ranunculoides Mœnch., vulgaire dans la plaine, remonte jusque dans la région des pâturages ct se retrouve encore au mois de Juin autour des chalets.

Le Pelusites officinalis Ioench., est au bord de l'Arve, à côté du pont de Belle-Combe.

Trois plantes communes dans les prairies et les ver. gers de la plaine se rencontrent également au pied de la montagne, ce sont: Primula grandiflora Lam. qui fleurit déjà en F.vrier, $P$. officinalis Jacq. qui s'élèvo assez haut sur les prairies montagneuses, $P$. stateolens Bertol. qui affectionne les taillis rocailleux du pied. Quant au $P$. veriubilis Goup., e'est évidemment un 
hydride entre $P$. grandiflore Lam. (P'. acaulis Jacq.) et $\boldsymbol{P}$. officinalis Jacq.

Une forme qu'on pourrait nommer $P$. acauli-suaveolens, d'après la nomenclature adoptée pour les hybrides, semble particulière au pied de Salève; les deux autres formes, $P$. acauli-officinalis Murel, Rapin et $P$. officinuli-acaulis Muret, Reuter, se rencontrent plutôt dans les verger's de la plaine. L'une se reconnaît à ses hampes, terminées par une ombelle de fleurs dirigées cn tous sens; la corolle est citrine, assez grande et à limbe plat; elle se rapproche du $P$. acaulis Jacq., et clle a quelquefois des pédicelles radicaux. L'autre a ses hampes terminées par une ombelle de fleurs à corolles plus petites, plus foncées et concaves; elle se rapproche du $P$. officinalis Jacq., mais les fleurs ne sont pas dir'igées d'un seul côté. Ces deux formes ne se rencontrent que dans les localités habitées par les parents; clles fleurissent quand le $P$. acuulis commence a passer et avant le $P$. officinalis. Tous les échantillons que j’ai examinés m'ont paru stériles.

Le $P$. variabilis Goup. n'est donc point une espèce légitime, c'est une forme intermédiaire présentant deus types, mais avec une foule de nuances qui les rattachent aux deux parents. Quant à la forme $P$. acuuli-suaveolens, c'est également un hybride du $P$. acaulis dacq. ct du $P$. suaveolens Bert., ce dernier remplace au pied de Salève le $P$. officinalis Jacq., dont il n'est peut-être qu'une variété.

Vers la fin du mois, une promenade du côté de Cruseilles fera trouver le Betula alba L., sur le revers méridional, et l'Ulmus montana Sin., dans les bois du pied de la montagne. 


\section{Mois d'Avril.}

Pendant ce mois, les excursions se borneront encore aux différentes localités du pied et du pourtour de la montagne.

Les espèces les plus printanières ont déji terminé leur courte carrière; cependant nous retrouverons la plupart de celles déja signalées, soit avec des fleurs plus avancées, soit en bon élat de fructification.

En prenant le sentier qui monte dirertement a Crevin depuis la ferme de l'Hôpital, on trouve au bord du ruisseau Allium ursinum L., commun dans tous les endroits humides du pied de la montagne; à droite, au-dessous du moulin, se cachent les touffes rosées et dépourvues de feuilles du Lathraea squamaria L. , parasite sur les racines des noyers.

Dans un verger, au-dessus du village de Bossey, fleurit le Petasites Reuteriana Jord., variété du $P$. vulgaris Mœench., dont il se distingue par des feuilles un peu différentes el développées en même temps que les fleurs.

Parmi les buissons et les taillis, entre Veyrier et le Coin, on récoltera Fraxinus elatior L., Carpinus betulus $\mathrm{I}_{\text {. }}$ et Salix grandifolia Ser., qu'on reprendra plus lard en fevilles bien développées. Çà et lả se trouvent: Buxus sempervirens L., commun dans la plaine des rocailles, Taxus baccata L. el Hippophac rhamnoides L., qui préfëre les rịves sablonneuses de l'Arve. 
Dans les endroits frais et abrités: Anemone nemorosa L., Viola sylvatica Fries., V. Riviniana Reich., Oxalis acetosella L., Orobus vermus L., Mercurialis perennis L., Arum vulgare L., Paris quadrifolia L., Carex montana L., C. praecox Jaq. et C. glauca Scop. dans tous les terrains humides et argileux.

Dans les taillis récemment coupés, surtout entre Crevin et Veyrier, se trouvent en abondance: Arclostapleylos officinalis W. et G. et Viola mirabilis L., dont les fleurs d'un bleu pàle ont un parfum plus fin et plus suave que celui de la violelte odorante.

Sur les arbres fruitiers, le Viscum album L. est en ffeurs et en fruits. Dans les haies fleurissent Ribes uva-crispa L., Prunus spinosa L. et P. fruticans Weih.

Au pied de ces mêmes haies se cache Viola multicuulis Jord., qui se distingue du V. odorata L., par ses stolons nombreux et radicants, par la forme de ses feuilles, par ses fleurs blanches à l'intérieur, mais colorées à l'extérieur et relevées de stries d'un violet foncé; elle est moins odorante que la violette ordinaire. Toujours à l'abri des haies, verdoie l'Adoxa moschatellina L. dont les feuilles exhalent, quand on les froisse, une faible odeur de musc, avec Lamium maculatum L. , L. purpureum L. et Glechoma hederacea L., qu'on retrouve à Mornex et à Monnelier.

Dans les broussailles, près de la funtaine de JulesCísar, quelques pieds de Pulmonaria officinalis L. , vivent en société avec $P$. tuberusa Sclir., Gr. et G.

Dans les cultures du village se répand Veronica Buxbaumii Ten., autrefois rare dans le canton et qui se montre aujourd'hui dans une foule de localités.

Çà et là dans les champs: Lamium amplexicaule. L. , Gagea arvensis Schultz. Koch, et Muscari racemo. 
sum Mill., qu'on relrouve dans les cultures et dans les vignes de Mornex.

Dans tous les prés se dressent les hampes lilas du Cardamine pratensis L., les liges en ombelles du Primula officinalis Jacq. et plus rarement du P. variabilis Goup.

Dans le bois de Veyrier: Malus communis Poir. et M. acerba Nerat.

En montant le Pas-de-l'Echelle, on trouve en abondance dans les débris rocailleux Hulchinsia petreu. Br.; plus haut it gauche, vers le sommel du sentier, Acer opulifolium Vill. et Salix migricans Fries. var?. eriocurpa Godet.

Autrefois, se tenail, pres de la source, un bon vien. qui vendait quelques rafıaichissements et qui, plus tard, se hornait it demander aux promeneur's un petit sou, ne serait-ce même qu'une centime. Lor'squ'il voyait passer un botaniste, il ne manquait pas de lui raconter comme quoi il apportait autrefois en ville les belles plantes de la monlagne, cntre autres la Rancune des Alpes... C'étail le Trollius Europeus, qu'un amateur lui avait dit se nommer la Renoncule des Alpes, et dont il avait ainsi défiguré le nom à sa façon.

Le Petit-Salève est riche en Vina minor L., si commune dians les haies et les bois de la plaine. Pent-être en parcourant avec soin cette partic de la montagne, pourrait-on retrouver le Ruscus aculeatus L. indiqué jadis an Petit-Salève, et dont M. Reuter possède un échantillon qui lıi a été donné comme provenant de celte localité.

Vers la fin du mois, une course au bas de la GrandeGorge fera trouver les Salix Pontederana Willd., Gaud. at S. Seringeana Gaud. Ces deux hybrides sont rares comme tous leurs congénères. M. Rapin a observi 


\section{7}

vers le Fort-de-l'Ecluse un pied femelle du Pontederana, dont il a cucilli des rameaux en bonnes fleurs ¿a mois de Mai. Le Seringeana a été signalé par M. Ie curé Chavin, dans le vallon des Usses, près des Bains de la Gaille; il existe en outre près de la Tuilerie de Chàtelaine et dans quelques localités du canton de Vaud; mais il n'y a que des pieds femelles dans nos environs.

Au bord de l'Arve, on récoltera des rameaux bien feuillés des Salix daphnoides Vill., l'une des belles aspeces de notre Flore, $S$. merpurea $L_{\text {.. }}, S$. incana Schr., el S. nigricans Fries. var. a leiocarpa Godet; dans les sables se trouvent : Anemonc rununculoides L., Carex peniculata L., C. nilida Host. et C. ampullaceu Good. 


\section{Mois de Mai.}

Pour épuiser toute la végétation de ce mois, l'un des. plus riches en plantes intéressantes, il faudra plusicurs herborisations faites a des intervalles variés.

En se dirigeant d'abord du côté du Coin, on visilcra le petit marais de Collonges, où l'on trouve: Valeriana dioica L., Taraxacum palustre DG., Pinguicula vulgaris L., Orchis latifolia L., O. incarnata L., - longtemps confondu avec le précédent dont il se distingue par sa floraison plus tardive et par son épi plus allongé qui dépasse les feuilles de beaucoup, Eriophorum ungustifolium Roth., Carex Hornschuchiana Hopp. avec sa var. $\beta$ xunthocarpa God., $C$. lepidocarpa Tausch., C. flava L., C. dioica L., C. Davalliana Sm., Equisetum Telmateya Ehrh. La plupart de ces plantes existent aussi au marais de Troinex et de Veyrier.

Au bord de la source sortant des éboulements du Coin, lleurit Cartamine amara L., qui se retrouve autour de la fontaine des Pitons.

Autour et derrière les grands rochers du Coin: Arubis saxalilis L., rare à Salève et plus abondante autour du Fort-de-l'Ecluse; Arenaria grandiflora L., qu'on retrouvera dans les talus d'éboulements au-dessus de Crevin et qui a disparu de son ancienne localité près du château de Monnetier; Sisymbrium acutangulum DC., qui existe également au pied des ro- 
chers surplombant le Pas-de-l'Echelle; enfin Ononis rotundifolia L., fréquente sur les éboulis dénudés audessus de Bossey et sous les voûtes supérieures du Petit-Salève.

En visitant avec soin et en plusieurs fois les'taillis, les éboulements, les talus, les monticules, les petiles mares et les rochers en place entre le Coin et Veyrier, on finira par trouver toutes les plantes dont voici la longue énumération.

D'abord les végétaux ligneux, à tout seigneur tout honneur. Les taillis sont composés d'un fouillis d'essences variées, telles que: Berberis vulgaris L., Acer Pseudo-Platanus L., A. opulifolium Vill., A. campestre L., Evonymus Europeus L., Rhamnus alpinus L., $R$. catharticus L., R. frangula L., Genista germanica L.. G. pilosa L., dont il n'existe que quclques pieds au-dessus de Crevin, mais qui est commun autour du Fort-de-l'Ecluse, Cytisus Laburnum L., Coronilla Emerus L., Cerasus avium DC., C. Mahaleb DC., plus abondant au Petit-Salève le long de la route d'Etrembières à Mornex; on le retrouve entre Archarnp et le Châble, Rosa pimpinellifolia L., a vulgaris God., Crategus oxyacantha L. et C. Oxyacanthoides Thuill. qui se distinguent, le premier par ses feuilles plus profondément découpées, par ses fleurs à un seul style et par ses fruits a un seul noyau; le second par ses feuilles à lobes plus obtus et moins profonds, par ses fleurs plus précoces à odeur désagréable, par ses fruits plus gros et à deux noyaux, Cotoneaster vulgaris Lindl., C. tomentosı Lindl., Amelanchier vulyaris Mœnch., Sorbus ancuparia L., S. aria Crantz, S. scandica Fries, S. Iorminalis Fries, Malus communis L., Ribes alpinum L., Comus sanguinea L., Vibumum Opulus L., V. Lantana L., Sambucus ra- 
cemosa L., ,S. nigra L., Lonicera xylosteum L., L. nigra L., plus abondant au-dessus d'Arehamp, $L$. alpigena L., Ilex aquifolium L., Daphne alpina L., surtout sur les blocs détachés de la montagne, Quercus racemosa Lam., Q. sessiliflora Sm., Q. pubescens Willd., Ulmus montana Sm., plulôt vers Archamp, Carpinus Betulus L., Juniperus communis L., Pinus sylvestris L., P. uncinata Ram. Gorl., au-dessus de Grevin, et Taxus baccata L.

Au pied et it l'abri de ces taillis, fleurissent: Dentaria digitata Lam., D. pinnata Lam., au-dessus de Crevin et d'Archamp, Viola mirabilis L., Orobus niger L., O. tuberosus L., Spircea aruncus L., Bellidiastrum Michelii Cass., Hieracium murorum L., avec quclques-unes de ses nombreuses variétés, telles que: H. nemorense Jord. et $H$. praecox Schultz., Galeobdolon luteum L., Melittis melissophyllum L., Euphorbia dulcis L., E. amygdaloides L., Convallaria verticillata L., C. polygonatum L., C. maialis L., Maianthemum bifolium DG., Paris quadrifolia L., Orchis galeata Lam., O. simia.L., O. purpurea Huds., Garex leporina L., C. gynobasis Vill., C. digitata L., C. ornithopoda Willd., C. alba Scop., C. pallescens L., C. sylvatica IIuds., Anthoxanthum odoratum L., avec sa var. $\beta$ villosum. Reích., enfin Sesleria corulea Ard.

Sur les petits mamelons dénudés, entre le Coin et Crevin, vivent en société: Helianthenum canum Dun., H. vulgare Gaertn., et ses deux variétés, Coronilla vaginalis Lam., Hippocrepis comosa L., Antlyyllis montana L., Rubus saxabilis L., Arctostaphylos officinalis W. et G., Globularia vulgaris L., G. cordifolia L., Orchis morio L., O. ustulata L., O. mascula L., Ophrys muscifera Huds., O. aranifera Sm., 0 . arachnites Reich., O. apifera Huds. 
Sur les talus du Pas-de-l'Echelie, autour des carrières de Veyrier et dans les éboulis entre ces carrières et Crevin: Aquilegia vulgaris L., Arabis alpina L., A. auriculata Lam. peu commune, A. hirsuta Scop., A. incana Roth., A. muralis Bertol., A. strictu Huds., et la forme intermédiaire A. hybrida Reut, A. Iurrita L.., Hutchinsia petraea R. Br., Kemera saxatilis Reich., Silene mulans L., Saponaria ocymoides L., Alsine tenuifolia Crantz, var. $\beta$ viscidula Gaud., Mcehringia muscosa L., Cerastium arvense L., ß strictum Reut., Saxifraga tridactylites L., Erinus alpinus L.

Le Saxifraga gramulata L. existe en petite quantité le long d'un sentier au-dessous des monlicules entre Veyrier et Crevin; la slation principale de celte jolie fleur est au bois de Bay ot aux environs de Vernier.

Derrière un rocher détaché de la montagne, ì gauche du sentier de la Grande-Gorge, se trouve Lunaria rediviva L., avec Muscari comosum L. ; la première, assez rare, est aussi au-dessus d'Archamp et de Pommier.

Au bord des petites mares qui occupent le pied des monticules du bois de Grevin, fleurit Viola pumila. Vill. et dans l'eau même, Ranunculus trichophyllus Chaix.

Sur les rochers et les éboulements au pied de la Grande-Gorge, se trouvent de rares échantillons du Scorzonera austriaca L.; il en exisle aussi au-dessus d'Archamp et sur le Vuache, et il est abondant sur les rochers du Bas Valais.

Près de Bossey, le long du ruisseau qui coule à côlé de la Ferme de l'Hôpital, il y a de beaux pieds de Carex maxima Scop. 
En faisant l'ascension des Pitons par le sentier dit de la Traversière, il faudra faire un détour pour récolter au-dessus d'Archamp et au bord de petiles mares Scorzonera humilis L., assez rare et découvert en cet endroit par M. Rapin en 1866; plus haut, parmi les éboulements situés au-dessous de grandes roches perpendiculaires, se trouvent deux stations de l'Atrugene alpina L. qui couronne de ses guirlandes élégantes, d'un bleu azuré, les Rhamnus alpinıs L., Sorbus scandica Fries, Lonicera nigra L., Sambucus racemosa L., Salix qrandifolia Ser., et les autres arbrisseaux dẻjà signalés.

J'engage les botanistes à chercher, au-dessous de cetle localité, Globularia nudicaulis L., indiqué avec doutc par M. Reuter, d'après M. Ducommun qui m'affirme cependant l'avoir trouvé à deux reprises différentes.

En reprenant le sentier qui se dirige vers la terme des Beulets, on récoltera Dentaria digitata Lam., D. pinnatu Lam., Primula clatior Jacq. et Cypripedium calceolus L., dont j'ai trouvé une fois dans une clairière un groupe de 14 individus la plupart à deux fleurs; cette belle Orchidée devient toujours plus rare, par suite de la chasse que lui font les enfants qui l'arrachent pour l'apporter au marché.

Dans les champs des Beulets, abonde Fumaria Chavini Reut., (F. Vaillantii Lois., d'après M. Rapin).

En montant dans les bois, on déconrre le Neottia nielus-avis L. qui croît dans la mousse. L'Actcen spicala L., le Myosotis sylvatica L. aux fleur's azurécs el l'Asperula odorala L., dont le parfum se développe par la dessication, vivent au pied des Abies pectinata DC. et A. excelsa DC. Sur les rochers fleurit Arabis alpina L. avec A. alpestris Schl.; vers la source des 
Pitons: Cardamine amara L. et Chrysosplenium alternifolium L. peu commun; antour des Pitons : Scleranthus bionnis Reut., Vaccinium Myrtillus L., V. Vitis-idnea L., Alnus viridis DG., Carex stellulata Good., C. vulgaris Fries.; sur les Pitons mêmes : $G$. tenuis Host. et $C$. sempervirens Vill., ce dernier se retrouve au sommet de l'entonnoir de la Grande-Gorge.

Dans les pàturages fleurit : Ranunculus gracilis Schl. (R. montanus Willd. $\beta$ gracilis Rapin) forme différente du $R$. montanus du Brison; dans les prairies se dressent les hampes du Trollius Europaeus L., du Cardamine pratensis L. et du Lychnis Flos-Cuculli L.; partout le gazon est émaillé des touffes azurées du Gentiane verna L; dans les endroits tournés au Nord et récemment abandonnés par la neige, percent les fleurs violettes, blanches et panachées du Crocus vernus All.; autour des mares, s'étalent les corolles vernissées du Caltha palustris L. et. du Ficaria ranunculoides Mœnch; sur les rochers s'épanouissent les pétales d'un blanc carné du Rosa pimpinellifolia L. $\beta$ spinosissima God.; les pâturages secs offrent : Arrhenaterum clatius M. et K., Festuca avina L. et Bromus erectus Huds.; enfin, au sommet de la Petite-Gorge et parmi le gazon : Carex humilis Leys.

En descendant à Monnetier, on trouve sur les pentes Doronicum Pardalianches L.: qui existe en outre dans une haie près de Veyrier et dans les taillis audessus de la Fontaine de Jules-César.

On cultive dans le vallon de Monnetier: Brassica oleracea L., B. Rapa L., B. Napus L., Faba vulgaris Mœnch., Vicia sativa L., Pisum sativum L, P. arvense L., Phaseolus vulgaris L., P. nanus L., etc.

Dans tous les champs, on trouve : Delphinium Consolida L., Fumaria officinalis L., Cardamine hirsuta 
L., Sisymbrium Thalianum Gaud., Sinapis arvensis L., S. Schkuhriana Reich. (var? du précédent), Alyssum calycinum L., Erophila verna DC. avec quelquesunes des espèces de Jordan (E. glabrescens, E. majuscula), Camelina sativa Crantz, Thlaspi arvense L., T. perfoliatum L., Iberis amara L., I. pinnata Gouan., Neslia paniculata Desv., Rapistrum rugosum All., Raphanus Raphanistrum L., Viola agrestis Jord., V. segetalis Jord. (var. du V. tricolor L.), Agrostemma Githayo L., Asperula arvensis L., Sherardia arvensis L., Senecio vulgaris L., Anagallis cœrulea Lam., A. phonicea Schreb. Veronica iriphyllos L., V. arvensis L., $V$. hederaefolia L., $V$. didyma Ten., Gr. et G.

Presque toutes ces plantes appartiennent à la plaine, elles sont cultivées en quelque sorte involontairement, et elles finiraient probablement par disparaitre avec la cessation de la culture des céréales et des légumes. On trouve également le long des haies et des murs des villages : Chelidonium majus L., Sisymbrium officinale Scop., Alliaria officinalis Andrz. el Lepidium campestre $\mathrm{R}$. Br.

Sur les rochers, près du château de l'Ermitage, fleurissent quelques échantillons du Potentilla rupestris L. bien plus maigres que les magnifiques pieds qui abondent au bois de Bay. En cherchant bien dans les éboulis, on retrouverait peut-être quelques touffes d'Arenaria grandiflora L. qui parait avoir suivi le sort de l'ancienne ruine dont le fronton portait l'inscription mélancolique : Nasci, pati, mori (naitre, souffrir, mourir).

Sous les voûtes du Petit-Salève : Sisymbrium Sophia L., aussi rare ici qu'il est commun en Valais, Ononis rotundifolia L., Anthriscus vulgaris Pers., Galium spurium L. $\gamma$ tenerum Gr. et G.(G. tenerum Schl.), Leucoium vernum L. et Bromus sterilis L. 
Près du cimetière du village, on trouve Cardamine Impatiens L., dont NI. Bernett m'a fait cueillir l'été dernier de magnifiques spécimens dans le bois de la Queuc-d'Arve, nouvelle localité pour cette plante rare aux environs de Genève.

En descendant le Pas-de-l'Echelle, on trouve près de la fontaine : Geranium lucidum L., abondant au MontGosse, Acer opulifolium Vill. et Salix nigricans Fries, $\beta$ eriocarpa God: en feuilles bien développées. Sur leṣ rochers qui dominent le sentier : Sisymbrium acutangulum DC. et Draba aizoides L. en fruits; le long du chemin : Erucastrum obtusangulum Reich. Koch; en bas, dans les graviers, on cherchera: Nardurus tenellus Reich. (Triticum Nardus DC. Gaud.), mignonne graminée que nous avons récoltée en quantité dans un champ près de Versoix. Dans la gravière de Veyrier : Vulpia Pseudo-myıtros Gay et dans les éboulis : $\boldsymbol{V}$. ciliata Link, beaucoup plus rare et qu'on retrouve, soit au bord de l'Arve, soit dans les sables d'Aïre.

Dans les champs arides et dans les prés secs: Cerastium viscosum L., C. semidecandrum L., C. brachypetalum Desp., C. glutinosum Fries., C. vulgatum L., Alsine temifolia Crantz, avec la var. $\beta$ viscidula Gaud., A. laxa Jord., Arenaria sphaerocarpa Ten., A. leptoclados Reich., qui se retrouvent sur les champs de la montagne. Sous Veyrier, le long de la rivière : Alsine hybrida Jord., Myricaria Germanica Desv., Hippophae rhamnoides L., et Typha minima Hop. qui refleurit souvent en automne. Sous Veyrier, dans une haie : Gagea lulea Schultz Koch, qui se retrouve près d'Etrembières. Vers Sieme, dans une haie également: Tulipa sylvestris L. difficile à trouver en fleurs, mais dont on peut faire une ample moisson dans les champs de Neydans, près Saint-Julien. 
Dans les prairies humides, autour du marais de Veyrier: Arabis sagittata $\mathrm{DG}$.

Dans le marais de Troinex, au bord de la route qui fait la limite du canton : Primula farinosa L. introduit. autrefois par M. Reuter et retrouvé par N. Ducommun, Gardamine Mutthioli Norett., cspèce qui paraît distincte du C. prutensis L. et qu'elle remplace en Piémont, Salix repens L., Orchis coriophora L., O. lalifolia L., O. imcarnata L.; malheureusement ces plantes tendent à disparaitre par le fait du drainage et des culures qui changent la nature de cette station.

Près de Veyrier, à la lisière du bois : Sorbus domeslica L.

Dans les champs à Troinex et près d'Evordes: Narcissus biflorus Gurt. ; à Crevin et Collonges, dans les prés : $N$. poeticus L. probablement échappé des jardins.

Une course à Cruseilles fera retrouver, sur le revers méridional, Betula alba L. et Alnus viridis DG.; dans les champs sablonneux du chalet des Avenières, Fumaria Chavini Reut., Scleranthus annuns L. et S. perennis $\mathrm{L}$.

Enfin, près d'Etrembières, dans la haie à gauche en montant à Nornex : Gagea lutea Sch. Koch.; au chemin des châtaigniers : Potentilla alba L., rare à Salève, abondante au bois de Bay et à celui de Promenthoux.

Entre Mornex et Reignier, dans une mare: Ramunculus puncistamineus Tausch. (R. Droueltii Reut. Rap. non Schultz. d'après Grenier). 


\section{Mois de Juin.}

En arrivant à Veyrier, on cherchera dans les champs : Reseda Phyleuma J., plante erratique existant cependant encore dans la localité indiquée par de De Saussure, au bord du Rhône sous Avully; dans les parties cultivées du marais de Troinex: Iberis amara L. et Orlayk grandiflora Hoffm.; dans les prairies artificielles: Gulium glaucum L. qui parait ou disparait suivant la provenance des graines; dans les champs arides: Pavaver dubium L., a Lecoqii Lamol. el $\delta$ collinum Bogen, Althaea hirsuta L. et Lathyrus hirsutus L.; dans la gravière: Agrostis interrupte L.; autour des carrières: Alsine fusciculala M. et K., Echinospermum lappula Lehm., Erinus alpinus L. et les Arabis en bons fruits.

En montant le Pas-de-l'Echelle: Trifolium alpestre L., Rosa mollissima Fries, R. subglobosa Sm., $R$. tomentella. Lem., Senecio viscosus L., Orobanche Teucrii Schultz., Allium sphacrocephalum L., Phleum Bechmeri Wib., Lasiagrostis Calamugrostis Link., Scleropoa rigida Griseb., Nardurus tenellus Reich., qui se tronve également dans les carrières de Nlornex, mais toujours en petite quantité.

Sous les voûtes du Pelit-Salève: Asperugo procumbens L., seule localité, pour nos environs, de celte plante commune en Valais. 
Dans les prairies, les champs et les cultures du vallon de Monnetier: Ranunculus arvensis L., R. repens L., R. acris L.. R. bulbosus L., Papaver Rhoeas L., P. Argemone L., Conringia orientalis Andr., erralique et rare (M. Bernelt), Arenuriu sphaerocarpa Ten. Jord., A. leptoclados Reich., - deux formes de l'A. serpyllifolia L., qui ont des caractères assez distinctifs et constants, tirés des tiges, des feuilles et des capsules Spergula arvensis L. rare, Gypsophila vaccaria Sibt. et Sm., Geranium pyrenuicum L., G. molle L., G. pusillum L., G. rotundifolium L., G. columbinum L., G. dissectum L., G. Robertianum L., Erodium triviale Jord., Oxalis Europaea Jord., Anthyllis vulneraria L., Medicago lupulina L., Melilotus arvensis Waltr., Trifolium arvense L., T. montanum L., Egopodium podagraria L., Cancalis dancoides L., Scandix Pecten-Veneris L., Galium tricorne With. rare, G. Spurium L., $\beta$ Vaillantii Gr. et Godr. rare, Valerianella auricula DC., V. olitoria Mønch., V. Morisonii DG., a leiocarpa God., Centaurea Cyynus L., Cirsinm arvense Lam., Sonchus arvensis L., Campanule rapunculus L., Convolvulus arvensis L., Anchusa Italice Retz, Linaria minor Desf., Rhinanthus alectorolophus Poll., Galcopsis praecox Jord., Avena fatua L., Lolium perenne L., L. strictum Presl., L. temulentum L.

On cultive en outre: Limum usitutissimum L., Medicago sativa L., Trifolium pratense L., Avena sativa L., A. orientalis Schr., Triticum vulgare Vill., T. monococcum L., T. Spelta L., Hordeum vulgare L., H. zeocriton L., et Secale cereale L.

Sur la pente du Petit-Salève, près du sommet et audessus du village: Mespilus Germunica L., découvert par M. Rapin, Orobanche Cervarice Suard; sur les points culminants: Scleranthus verticillatus Reich., 
Rosa coronata Crep., Phyteuma spicata L., var. flore caruleo et Vicia temuifolia Roth.

Autour du village: Tilic platyphyllos Scop.

En montant aux Treize-Arbres, on fera collection des diverses espèces, formes ou variétés de ronces et rosiers. Ces genres, épineux à tous égards, exercent la sagacité des botanistes de nos jours; l'école synthétique s'en tenant aux espèces linéennes, repousse les nouveautés de l'école analytique, et j'avoue qu'il n'est pas toujours facile de se reconnaitre au milieu des nomibreuses espèces créées par les novateurs. Néanmoins toutes ces formes critiques existent dans la nalure, on les rencontre à chaque pas, il faut bien les examiner, les décrire et a!ı besoin les nommer, quilte à les rattacher à l'un des types admis par les auteurs. G'est vraiment dommage qu'on ne puisse pas les supprimer, on aurait là un moyen commode et expédilif de tourner la difficulté.

En montant sur le Grand-Salève, on trouve: Rubus dumetorum W. et $\mathrm{N}$., avec la var. a virescens Mere., il appartient au groupe des Rubi triviules Nerc., dont fait partic le $R$. caesius L. Les $R$. hirtus W. et N., et $R$. Guntheri W. et N., appartenant au groupe des Rubi glandulosi Nerc. Les $R$. rusticamus Nerc., $R$. elongatus Merc., et $R$. albidus, $\beta$ Saluevue Merc., appartenant au groupe des Rubi fruticosi discolores Merc.

Passons à la nombreuse tribu des liosicrs qui offre: Rosu alpina L., avec ses trois var. $\beta$ pyrencica, DG. \% lagenariu Vill. of aculeatu Ser. commune surtout sur les talus d'éboulements de la face occidentale, $R$. Saburuda Rap. sur toutes les sommités, avec le $R$. coronata Crep. qui, d'après M. Rapin, n'est qu'une variété de son $R$. Subauda. Sur les pentes qui domi- 
nent le valon de Monnetier: $R$. vestita God., plus abondante au-dessus d'Archamp; sur le sommet ver's les Treize-Arbres et la Grande-Gorge: $R$. Salaevensis Rap., R. rubrifolia Vill., $R$. alpestris Rap. Reut. qui existe aussi auv Pitons, $R$. coriifolia Fries, dont la var. biserrata Reut. habite la Croisette et Monnetier, $R$. sphaerica Gren., avec $R$. biserrata Mér. Bor.

Ces rosiers el d'autres encore se retrouvent en abondance sur les talus d'éboulements, surtout an-dessus d'Arehamp; il faut les récolter le matin au moment où les corolles viennent de s'épanouir et les mettre de suite en papier; plus tard il faut les prendre en fruils verts, puis en fruits mûrs, afin d'avoir tous les caractères qui permettent de distinguer les espèces. Pour compléter les échantillons, il convient de sćcher des drageons de l'année. Ces mêmes observations s'appliquent en partie au genre Rubus.

On trouvera dans les pàturages du sommet: Trollius Europeus L., Cerastivm vulgatum L. $\beta$ alpinum Gr. ct G., Dianthus Garthusianorum L., Geranium sylvaticum L., Alchemilla vulgaris L., A. alpina L., Sanguisorba montana Jord., Carum carvi L., Astrantia major L., Pimpinella magna L., avec la var. $\beta$ rosea Koch, Gnaphalium dioicum L., Hypochaeris maculata L., Lcontodon hispidum L., Taraxacum lacvigatum DG., Hieracium auricula L. forma alpina, Campanula glomerata L., Orchis globosa L., Nigritella angustifolia Rich., (0. nigra Scop.) Phleum pratense L., $\gamma$ nodosum Gaud., Poa alpina L., Nardus stricta L.

Dans les champs des Treize-Arbres, outre la plupart des espèces de Monnetier, Viola Sagoti Jord. Reut. (V. tricolor L. var.) et Galeopsis intermedia Vill.

Au sommet de la Petite-Gorge: Carex humilis Leys. 
Autour de l'entonnoir de la Grange-Gorge: Ramunculus Thora L., qui ne se trouve que dans cetle localitẻ, Carex sempervirens Vill. et Selaginella spinulosa A. $\mathrm{Br}$.

Vers le Trou dit de Briffaut, au-dessus de la Croisette, est la seule station de Dryas octopetala L.

Le long du chemin qui monte à la Croisette: Avena pratensis $\mathrm{L}$.

$\mathrm{Au}$ bord d'une petite mare, creusée dans le sable, entre la Groisette et les Pitons : Sedum villosum L., qui ne se retrouve pas ailleurs dans nos environs; en revanche, il est commun dans les prairies humides du plateau du Simplon.

Sur la chaine des Pitons, dans les pâturages el le long des ruisseaux : Ranunculus aconitifolius L.; dans les bois de sapin du sommet: $R$. platanifolius L.; ces deux espèces Linéennes, confondues par la plupart des auteurs, sont parfaitement distinctes.

Dans les pâturages : Ranunculus gracilis Schl. en fruit, Viola canina L. et var. minor Reut., Potentilla nerna L., P. aurea L. Koch., P. Jurana Reut. (M. Rapin réunit, sous le nom de P. alpestris Hall. fil., les P. Salisburgensis Hænk., P. aurea Gaud. et P. Jurana Reut.), Scleranthus biennis Reut., Campanula rhomboidalis L., Rhinanthus angustifolius Gm.

Sur un espace tourbeux el marécageux, derrière le Grand-Piton : Alnus viridis DG., Eriophorum vaginatum L., Carex stellulata Good., C. vulgaris Fries.

Dans les prairies humides: Viola nemoralis Jord. qui n'est qu'une variété du V. canina L., Sagina Linnaei Presl. rare, Polygala amara L. Koch., Parnassia palustris L., Lotus corniculatus L., Tetragonolobus siliquosus Roth., Geum rivale L., Potentilla Tormentilla Nestl., Veronica serpyllifolia L. La plupart habitent la plaine, mais aiment à s'élever. 
Sur les rochers des Pitons : Arabis serpyllifolia Vill. dans les fentes du côlé occidental, seule localité: Cette espèce, qui préfère les montagnes calcaires, se trouve cependant sur les roches molassiques compactes du sommel des Voirons. Arubis alpestris Reich., Geum montanum L. rare et seulement sur le Grand-Piton, Saxifraga aizoon L., Veronica fruliculosa L. rare, Hierucium villosum L. peu abondant, Orchis sambucina L. rare, Luzula flavescens Gaud., Carex temuis Host., C. sempervirens Vill., Poa alpina L. $\beta$ brevifolia Gr. et G. et Festuca glauca Lan.; ces dernières espèces occupent le sommet du Grand-Piton.

Dans les bois de sapins, entre les Pitons el Cruscilles, on trouve : Thalictrum uquilegifolium L., Ranunculus nemorosus DG., Gardamine syluatica L., Lychnis diurna Sibt., Mahringia trinervia Clairv., Stellaria nemorum L., Geranium sylvaticum L. , Saxifraga rolundifolia L., Lysimachia nemorum L., Veronica montanu L., Luzula. flavescens Gaud., L. nivea DG.

Aux environs de Cruseille, dans les champs sablonneux : Bunias erucugo L., Suyina procumbens L. et Scleranthus perennis L. qui se retrouve aux Voirons au-dessus de Bonne; dans les pàturages: Arnicu montana L. rare à Salève.

Près de Pommier, M. Rapin avait trouvé jadis Turritis glabrce L., qui a été r'evu depuis par M. le curé Chavin dans le vallon des Usses, près des Bains de la Caille.

En s'élevant sur le sentier pittoresque qui conduit aux Pitons depuis le Chàble et Pommier, on récoltera: Rosa Reuteri God. var. foliis biservatis Reul., Tiliu. intermedia DG., T. microphylla Vent., Chacrophyllum. cicutaria Vill., Campanula patula L., et la plupart des autres espèces qui viennent d'être indiquées. 
Près de la ferme des Beulets croissent: Rubus cuesius L., $R$. vusticanus Here, $R$. Iducus L. avec la variété hybride caesio-idaeus Merc., Rosa spinulifolia Dem., R. vestita God., R. montana Chaix., R. rubri'folia Vill., R. marginata Walr.

Au-dessus d'Archamp se rencontrent : Evomymus Europaeus L., Rhamnus alpinus L., Rubus nemorosus Gr. et G., R. discolor W. et N., Rosa pimpinellifolia L., $R$. alpina L., $R$. alpino-pimpinellifolia Reut., $R$. montuna Chaix., tous deux au-dessous du rocher de la Serratula, R. Salaevensis Rap., R. spinulifolia Dem., $R$. vestita God., R. rubrifolia Vill., R. marginata Wallr., $R$. subglobosa Sm., R. Reuteri God., R. coriifolia Frics., R. tomentella Lem., R. rubiginosa L., var. nemorosa Reut., R. micrantha Sm., Sorbus hybrida L., S. Scandica Fries.

A l'ombre de ces mêmes taillis: Fragaria vesca L. partout, $F$. collina Ehr. Valeriana tripteris L., V. montana L., Campanula persicifolia L., Digitalis lutea L., D. grandiflora Lam., Veronica TeucriumL., V. urticaefolia L. et Lilium Mariagon L.

Dans les places humides et marécageuses : Orchis Traunsteineri Saut., Epipactis palustris Crantz Limodorum abortivum Sw., Tofielda palustris Huds. Schonus nigricans L., Scirpus pauciflorus Light., S. compressus L.

Dans les prairies: Orchis conopsea L., O. odoratissima L., O. bifolia L., O. virescens Zollik.

Au-dessus du hameau du Coin, le long du sentier de la Croisette : Rosa Andeyavensis Bast, dans les haies, R. canina L. $\beta$ senticosa God. $\gamma$ sarmentosa God. o collina Gaud.; plus haut: Avena pratensis L.

Au-dessus de Grevin et sur les rochers à droite de la Grande-Gorge, on trouvera: Thalictrum minus L. 
II saxatile Gaud., Hieracium staticefolium Will., $\boldsymbol{H}$. pilosello-praealtum Schultz., rare, $H$. glaucum All., $H$. flexuosum Waldst. et K., peu commun, Trinia vulgaris DG., Rumex aeetosella L., Stipa pennata L.

Dans les talus d'éboulement au bas de la GrandeGorge : Helianthemum fumuna Mill., Lathyrus pretensis L., L. sylvestris L., Vicia sepium L., Rosa pimpinellifolio-alpina Rap., rare, $R$. tomentosa Sm., R. Chavini Rap., R. canina L., R. sepium Thuil., avec la var. o pubescens Rap., Reut., $R$. sphaerica Gren., R. Andegavensis Bast., Lonicera periclymenum L., L. xylosteum L., L. migra L., L. Alpigena L. , Galium sylvestre Poll., G. sylvuticum L., G.commutatum Jord., Leontodon hastile L., Leucanthemum corymbosum Gr. el G., L. vulgare Lam. L. montunuи Koch., Jlex aquifolium L., Ligustram vulgare L., Vince-toxicum officinale Monch., Purietaria erecte II. et K., Spiranthes aestivalis Rich., Epipactis latifolia All., E. rubiginosa Gaud., Cephalanthera grandiflora Balb., C. ensifolia Rich., C. rubra Rich., Ornithogalum pyrenaicum L., Lilium Martagon L., Anthericum Liliago L., A. ramosum L., Luzula maxima DC. , L. niver DC., Milium effusum L. dans les taillis, Poa nemoralis L. $\beta$ rigidula Gaud., Melica uniflora Retz, M. mutens L., M. Nebrodensis Parl., dans les endroits secs et chauds.

Dans les prés arides: Rhinanthus major Ehrh.

En continuant du côté de Veyrier, on trouvera de plus : Aconitum lycoctonum L., Arabis Turrite L., Polygala comosa Schr., P. vulgaris L., moins commun, Dianthus prolifer L., D. rupicola Jord., Linum tenuifolium L., L. calharticun L., qui se retrouve sur les sommilés, Vitis vinilera L., subspontané, Geranium sanguineum L., Lotus comiculatus L. et Tetragonolo- 
bus siliquosus Roth. déjà trouvés sur les prairies élevées, Trifolium rubens L., T. ochrolencum L., Rosa mollissima Sm., R. tomentosa Sm., R. monticola Rap., $\varepsilon$ cinerea Rap., $R$. mbiginosa L., R. umbellata Leers. Bor., R. sepium Thuil., R. arvensis Huds., Epilobium montanum L., Sanicula Europaea L., Galium montanum Vill., Lactuca muralis Fresen., Prenanthes purpurea L., Cynoglossum officinale L., Nelampyrum pratense L., Prunella laciniata L., P. grandiflore Jaeq., Daphne alpina L.

Dans une mare all-dessus de Crevin, j’ai trouvé $R a$ munculus divuricatus Schr., qui existe à Versoix et à Genthod.

Dans les marais de Bossey et de Troinex : Nymphaea alba L., Polygala Austriaca Crantz., Lotus temis Kil., Hieracium priealtum Vill., Menianthes trifoliala L., Pelicularis palustris L., Euphrasia montana Jord., Utricularia minor L., Liparis Loeselii Rich. rare, Schonus nigricans L., Cladium Mariscus R. Br., Scirpus panciflorus Light., Carex remota L., Triodia decumbens P. B.

Dans les fossés du marais de Veyrier : Potamageton plantagineus Ducr.

Les amateurs de rosiers trouveront dans le bois de Veyrier : R. Gallica L., R. Gallico-umbellate Rap., R. hybrida Schl. Gaud. (R. gallico-arvensis Reut.), $R$. Gallico-canina Reut. el $R$. umbellata Leers. Bor.

Une promenade de Monnetier à Nornex fera trouver sur les rochers, à mi-chemin : Rubus cuneifolius Nerc., R. Collinus DC., avec la variété hybride décrite par Mercier, enfin Plantago Cynops L., rare et spécial au Nont-Salève.

Les vieux murs de Mornex se garnissent de touffes de Linaria cymbalaria Mill., qui paraît se naturaliser complétement. 
Si l'on pousse jusqu'à Reignier, on cherchera dans les champs Nigella arvensis L., plante erratique qui. se trouve quelquefois dans les moissons avec Matrican ia Chamomilla L.

Dans les haies on trouve Cerasus Padus DG.

Enfin une course aux bains de la Caille fera trouver : Barbarea arcuata Reich, découverte par M. l'abbé Delavay, près d'Allonzier, entre l'église et le burcau de la douane, Turritis glabra L., dans le ravin des Usses, Evonymus latifolins L., déconvert par M. l'abbé Delavay dans des broussailles rocailleuses au-dessus de l'église d'Allonzier, entre ce village et Avregny, Primula auricula L., que j'ai récolté sur les rochers de la Rive gauche, au-dessus des bains, enfin Rosa felidu Bast., an bois de Barioz, d'après M. le curé Chavin ef M. l'abbé Puget. 


\section{Mois de Juillet.}

Les herborisations de Juillet compléteront celles du mois précédent, tout en fournissant une ample moisson de plantes nouvelles.

Une excursion à la partie méridionale de Salève, fera trouve près du Châble :'Geranium palustre L., an bord d'une mare, Glyceria plicula Fries., dans les fossés, Equisetum liyemale L., $\beta$ paleaceum Dœl. Bern., dans les endroits humides.

A Pommier, daris les cours de l'ancienne Chartreuse, Impatiens noli-tangere L., rare dans nos environs, Campanula patula L. et Atropa Belladona L., dans les laillis. En s'élevant sur le flane de la montagne, on trouve dans les bois: Chaerophyllum cicutaria Vill., Anthriscus abortivus Jord. ef Festuca sylvatica Vill.

A Cruseilles, dans les champs sablonneux : Scleranthus perennis L. et sur les murs : Epilobium colli. num Gmel.

Près de la Caille, dans le bois de Barioz : Rosa foetida Bast., Pyrola chorantha Sw., découverte par M. l'abbé Puget, Dipsacus luciniatus L. trouvé par M. l'abbé Delavay, au-dessous de Cernex, au bord de la rivière des Usses, Comarum palustre L. près du bureau de la douane du village d'Allonzier (M. l'abbé Delavay) et Potentilla caulescens L., sur les rochers du vallon des Usses, au-dessus des bains de la Caille. (M. le curé Chavin.) 
En montant aux Pitons, depuis Gruscilles ou depuis Pommier, on trouvera dans les bois : Ranunculus lamuginosus L., Stellaria nemorum L., Vicia sylvatica. L., Angelica sylvestris L. Adenostyles albifrons Reich., Soyera paludosa Godr., Hieracium elatum Fries., Campanula trachelium L. avec la var. $\beta$ dasycarpa Gren., Pyrola minor L., Lysimachia nemomum L., Veronica montana L., Molampyrum sylvaticum L., Corallorhiza imnata R. Br., rare, dans la mousse au pied des sapins, Calamagrolis montana DC., Milinm. effusum L., Trisetum farescens P. B., Poa nemoralis L., $\beta$ rigidula Gaud., Bromus asper L., Elymus Europaeus L., Equisetum sylvaticum L.

Dans les pàturages: Ranunculus aconitifolius L., R. acris L., Geranium sylvaticum L., Genista sagittalis L., Trifolium medium L., T. pratense L., Alchemilla vulgaris L., A. hybrida Willd., A. alpina L., Astrantia major L., Garum Carvi L., Pimpinella magna L., var. rosca Koch, Hypochaeris maculata L., Grepis biennis L., Hieracium auricula L. formu alpina, Girsium acaule L., Heracleum Sphondylium L., Gentiana lutea L., Thymus serpyllum L., Thesium pratense Ehrh., T. alpinum L., Orchis globosa L., Gymnadenia albida Rich., Platanthera viridis Lindl. Pluleum alpinum L., Keleria cristala Pers., Poa alpina L., Nardus stricta L. et Botrychium Lunaria Sw.

Sur les Pitons et aux alentours : Polygala alpestris Reich., Sagina Linnaei Presl., Stellaria uliginosa Murr., IIypericum quadrangulum L., Rosa alpestris Rap., Scleranthus biennis Reut., Myrrhis odorata Scop., rare, Homogyne alpina Cass., Erigeron alpinus L., rare et sur le Grand-Piton, Crepis aurea Cass., rare, Hieracium Pseudo-Cerinthe Koch, H. pulmonarioides Vill. H. Ligusticum Fries., H. dentatum IIopp., B. Salac- 
vense Rap. sur le versant Nord-Est du Petil-Piton, Campanula rhomboidalis L., Vaccinium Vitis-Idaea. L., Myosotis sylvatica Ehrh., Veronica fruticulosa L. rare, Rhinanthus angustifolius Gmel., Calamintha alpina Lam., Galeopsis Reichenbachii Reut., Plantago alpina L. rare, Luzula maxima DC., Deschampsia flexuosa Griseb. sur les couches sidérolitiques, Poa alpina L. $\beta$ brevifolia Gr. et G., Festuca glauca Lam.

Près du hameau de la Croisette et dans les pâturages sablonneux : Pedicularis uberosa L. rare, trouvé par MM. Rapin et Chavin. Sur les rochers : Hieracium caesium Fries., qu'on retrouve vers les Treize-Arbres. Dans les prairies : Orchis conopsea L., Nigritella angustifolia Reich. et $N$. nigro-conopsea Reich. hybride des deux précédents, rare et découvert par M. Rapin.

Autour du trou de Briffant: Dryas octopetala L.

Au Grand-Salève, dans les taillis et sur les rochers: Rubus hirtus W. et N., R. Guntheri W. et N., $R$. discolor W. et N., R. elongatus Merc., R. albidus Merc. B Salaevae Merc., Rosa Sabauda Rap., Lycoporlium selago L. et Selaginella spinulosa A. Br., ces deux dernières dans les pâturages. Autour de la GrandeGorge : Ramunculus Thora L en fruits et Lycopodium clavatum L. rare. Sur les rocher's des Treize-Arbres : Hieracium caesium Fries. M. Bernett a trouvé sur les rochers entre la Grande et la Petite-Gorge, ainsi que dans l'entonnoir de cette dernière, H. Pseudo-Cerinthe Koch. rare.

Dans les champs des Treize-Arbres : Galeopsis intermedia Vill.; autour de tous les chalets végètent avec Iuxuriance les espèces qui suivent l'homme, telles que: Capsella Bursa-Pastoris Vent., Lotus corniculatus L., 
Silaus pratensis Bes., Veronica hederaefolia L., Plantago major L., P. media L., Chenopodium BonusHenricus L., Rumex obtusifolius L., R. conglomeratus Murr., Urtica dioica L., U. urens L., Poa amnua L., Festuca pratensis Huds., etc.

Sur les pentes du Grand-Salève, en descendant à Monetier: Clematis Vitalba L. et Vicia sylvatica L.

Sur les rochers du Petit-Salève et sur les assises supérieures dı Grand, à l'endroit d'où l'on a extrait les pierres du pont de Carouge: Hieracium andryaloides Vill. rare et spéciale à notre montagne, $H$. Lignesticum Fries., II. amplexicaule $\mathrm{L}_{\text {.. }}$ H. mulmonarioides Vill., qu'on retrouve au-dessus des carrières de Monetier.

Sur les flanes du Petit-Salève: Verbascum nigrum L. et Orobanche Cervariae Suard.

Dans les champs du vallon : Trifolium arvense L., Dancus Carota L., Linaria spuriu Mill. L. minor DesL., L. elatine Mill., Odontites rubre Gr. et G., Galeopsis angustifolia Ehrh.

On cultive: Solumum tuberosum L., Spinaciu incrmis Nœnch., S. spinosa Mench., Bela vulgaris L., Atriplex hortensis L., Polygonum Fagopyrum L., ... etc.

Dans le village, autour des fumiers et au pied des murs: Atriplex patula L., Chenopodium hybridum L., G. murale L., C. album L. avec ses variétés, C. polyspermum L., C. vulvaria L., G. glaucum L., Rumex pulcher L., R. crispus L., R. acetosa L., etc.

En allant à Mornex on trouvera près du village: Mentha rotundifolia L., et au chemin des châtaigniers: Trifolium aureum Poll. avec Jasione montana L.

Près d'Etrembières: Foniculum officinale All. et Lactuca dubic Jord. au bord des vignes; Scrophularia Balbisii Ilorn. le long des ruisseaux. Dans les 
sables d'Arve, vers l'embouchure de la Nenoge : Hieracium Florentinum All.

Au pas de l'Echelle on trouve : Potentilla petiolulata Gaud., Senecio flosculosus Jord., Centaurea Scabiosa L. $\beta$ petrophila Reut. et Ilicracium Jacquini. Vill.

A Veyrier et à Troinex: Sambucus Ebulus L. dans les taillis, Orlaya grandiflora L. et Stachys arvensis L. dans les champs, Equisetum variegatum Schl. dans les sables au bord de l'Arve, Epilobium Lamii Schuliz, Laserpitium pruthenicum L., Campanula cervicaria L., Agrostis vulgaris With, var. mumila Reut. dans le bois de Veyrier.

Dans les petits marais de Troinex, Crevin, Bossey, Collonges. : Drosera longifolia L., D. obovata Koch., Parnassia palustris L., Galium boreale L., G. elongatum Presl., Utricularia vulgaris L., U. minor L., Triglochin palustre L., Epipactis palustris L. Spiranthes aestivalis Rich., Tofieldia palustris L., Juncus obtusiflorus Ehrh., Allium carinatum L., Equisetum palustre L., E. limosum L., E. hyemale L., Nitella capitata Ag., N. opaca Ag., N. polysperma A. Br., $N$. glomerata Ag., Chara hispida L. dans les petiles mares au-dessus de Grevin.

Dans les bois ef taillis du pied: Molinia cocrulea Mœnch. et M. littoralis Host., Hypericum montanum L., Epilobium spicatum Lam., Galium sylvaticum L., Leucanthcmum corymbosum Gr. et G., Hieracium nemorense Jord., H. boreale Koch., Clinopodium vulgare L., Stachys recta L., Betonica officinalis L., Teucrium Scorodonia L., Epipactis latifolia All., E. rubiginosa Gaud.

Vers Collonges: Sambucus Ebulus L., Circaea Lutetiana L., Carum bulbocastanum Koch. dans les champs, 
Mentha viridis L., M. sylvestris L., M. candicans Crantz. qui s'éleve assez haut sur la montagne le long des ruisseanx.

Au-dessus du Coin, dans un endroit marécageux : Euphrasia uliginosa Ducom., Reut.

Au-dessus d'Archamp: Tilia intermedia DG., Heracleum montanum Schl., Centaurea montana L., peu commune, Pyrola rotundifolia L., P. secunda L., $P$. media Sw., rare et seulement dans la localité de l'Atragene, Pinguicula alpina L., découvert par N. Bernett dans un seul endroit marécageux, Goodyera repens R. Br., rare ici, mais abondant au pied des Pins du Bois du Vengeron, Agrostis alba L., y gigantea Reut. et Festuca gigantea Vill.

Au pied des grandes roches perpendiculaires: Rosa montana Chaix., Serratula mudicaulis DC. très-rare et dans cette unique localité, Hicracium glaucum All., H. lanatum Vill. rare et peu abondant, Cynoglossum montanum Lam. peu abondant, et Festuca tenuifolic Sibt.; à gauche de ces mêmes roches, en se dirigeant vers la Croisette, dans un bois de sapins, M. Rapin a trouvé Rumex arifolius All. qui n’avait pas encore été signalé à Salève.

Dans les plaines argilleuses et humides au-dessus du village d'Archamp: Centaurea Jacea L., Plantago serpentinu, Vill., Orchis conopsea L., O. odoratissima L.

Au chemin de la Croisette: Vicia dumetorum L. ct V. Cracca L., commune surtout dans la plaine.

Enfin, en parcourant les talus d'éboulements et les rochers en place entre Veyrier et le Coin, on trouvera: Thalictrum minus L. II saxatile Gaud. en fruits, Polentilla potiolulata Gaud. contre les rochers à gauche de la Grande-Gorge, Sedum reflexum L. avec la var. $\beta$ glaucum Koch. S. anopetalum DC. surtout aux éboulements 
du Coin, S. dasyphyllum L., S. album L. S. acre L., S. sexangulare L., Pimpinella saxifraga L., Athamantha Cretensis L., Laserpitium Siler L., L. latifolium L., Adenostyles alpina BI. et F., Leucanthemum. montanum Koch., Garduus defloratus L., Leontodon hastile L., Hieracium glaucum All., H. flexuosum W. et K., rare, H. amplexicaule L., H. pulmonarioides Vill., au bas de la Grande-Gorge, H. Ligusticum Fries, Campenula rapunculoides L., C. subrumulosa Jord., Cyclamen Europacum L., qui recouvre de ses fleurs roses et parfumées presque toutes les rocailles du pied, Gentiana crusiata L., Orobanche Galii Dub., O. Teucrii Schultz, surtout au pas de l'Echelle, O. Scabiosæ Koch. et 0 . Laserpitii Sileris Rap. tous deux au bas de la Grande-Gorge, Verbascum Thapsus L., Digitalis grandiflora Lam., Galeopsis angustifolia Ehrh., Brunella laciniala Lam., B. grandiflora Møench., Teucrium Chamaedrys L. et T. montanum L. 


\section{Mois d'Août.}

La saison s'avance et bon nombre de plantes ont déjà disparu; nous trouverons néanmoins encore pendant le mois d'Août quelques espèces intéressantes et surtout des échantillons on bons fruits des plantes récoltées précédemment.

Dans les bois de Veyrier on cherchera: Hypericum humifusum L., Peplis portula L., Selinum carvifolia L., Laserpitium pruthenicum L. et Centunculus minimus L. dans les clairières humides, avec Chloru serotina Reich., qui se retrouve au bord de l'Arve près d'Etrembières.

Dans les champs: Filugo arvensis L., F. gullicu L. peu communs, tandis que $F$. spathulata Presl. et $F$. germanica L. Gaud. sont très-abondants.

Dans le village même an pied des murs : Pulicariu vulgaris Gærtn. rare, et Crepis foetida L.

Dans la gravière: Polycnemum arvense L. et P. majus A. Br.

Dans le marais de Veyrier et de Troinex: Gulium uliginosum L. et Polygonum lapathifolium. L. $\gamma$ incanum Reut.

Dans les parties défrichées du même marais, sur les racines du chanvre: Phelipaea ramosa C. A. Mey qu'on retrouve à Mornex.

Sur le Petit-Salève : Peucedanum Cervaria Lap. Koch et Senecio Jacobaè I., ce dernier au Mont Gosse.

Dans les cultures et le long des murs des village de Mornex et de Monetier; Malva sylvestris L. et M. roiundifolia $\mathrm{L}$. 
En montant au Treize-Arbres: Lappa intermedia Reich. qui diffère du L. major Gæertn. par ses gros capitules disposés en grappe le long de la tige.

Sur les pâturages de la montagne: Gnaphalium sylvalicum L., Cirsium eriophorum Scop., Carlina acaulis L., Leontodon autumnale L., Gentiana campestris L.

Dans les marais du sommet: Potamogeton densum L., $\beta$ lanceolatum Koch.

Sur les rochers du Piton : Hieracium villosum L. et H. dentatum Hopp., $\beta$ Salaevense Rap., Calamintha alpiua Lam.

Au-dessous des Pitons, avant d'entrer dans les bois : Hieracium melanotrichum Reut. rare et belle espèce, voisine du $\mathrm{H}$. boreale Koch.

Sous les Sapins: Senecio Fuchsii Gmel., Lappa major Gærtn., Stachys alpina L., Monotropa Hypopytis L. $\alpha$ glabra et $\beta$ hirsuta God.

Au-dessus d'Archamp: Cephalaria pilosa Gr. et G., Calamintha nepetoides Jord., Stachys alpina L.

Vers Collonges et le Coin: Sedum purpurascens Koch, Senecio Jacobaea L., Salvia glutinosa L., Euphrasia cricetorum Jord. et Blitum virgatum L. rare.

Au marais de Bossey: Enanthe Lachenalii Gm.

A Crevin, dans les champs: Anthemis Cotula L. ; dans les taillis: Calaminthu ascendens Jord.; parmi les rocailles: Seseli bienne Crantz, et le long d'un ruisseau, près de la Grande-Gorge: Saxifraga aizoides L.

Dans les bois entre Grevin et Veyrier: Knautia sylvatica Dub., Scabiosa succisa L., Cephalaria pilosa Gr. et G., Lactuca muralis Fresen. Koch., Prenanthes purpurea L., Hieracium umbellatum L., Campanula aygregata N. et B., Origanum vulgare L.

Parmi les rocailles et dans les endroits secs: Aster 
amellus L., Carlina vulgaris L., Callume vulgaris Salish. jusque sur les sommilés, Gentiana Germanica L., Erythracu Centunrium Pers. : Euphrusiu cumpestris Jord., E. cuprueu, Jord., E. Salisburgensis Funk.

Dans les lieux humides: Gentiana ciliata L., abondante au chemin des Chàtaigniers, entre Etrembières et Mornex.

Une course à La Caille fera trouver dans le vallon des Usses : Sedum maximum Koch, et au pied des rochers qui dominent les Bains: Cyclamen Hederuefolium. Ait. non Ten.

En même temps on récoltera près de Cruseilles, dans les taillis: Hieracium tridentatum Fries, H. boreule Koch y curvidens Gr et Gr. et $H$. melanotrichum Rout.

Derrière le village, dans des champs humides: $l l y-$ pericum humifusum L. $\beta$ Liollardi, DC:

Dans des champs sees of ablonneux : Filago minima L. et Euphrasia ericetorum Jord.

Près du Chable, dans les taillis : Hierucium varum Jord.

Dans les bois de Viry qu'on devrait visiter plus souvent: Isnardiu pulustris L., \& Peplis portula L., Spurganium simplex lluds. et $S$. minimum Fries. Houvés par M. le curé Chavin. 


\section{Mois de Septembre.}

Pendant ce mois et pour le reste de l'automne il n'y aura plus à récolter qu'un petit nombre de plantes de l'arrière-saison, des Fougères et el surtout des specimens en bons fruits d'Ombellifëres et de Rosiers.

On trouvera dans les terrains argilleux el les Bruyères du pied de la montagne: Spiranthes untumnalis Rich.; dans les prés secs: Andropogon Ischaemum L. ; dans les prairies: Colchicum autumnale L.

Au marais de Troinex : Polystichum Thelipteris, 1)C., peu abondant et fructifiant rarement.

Au-dessus du château d'Etrembières et au chemin des Chàtaigniers: Asplenium Adiunthum nigrum L.

II. Rapin a trouvé près de la Mûre sur les clòtures sèches, en picrres cristallines : Celerach officinarum C.B., et sur les blocs erratiques au-dessus du même village: Asplenium septentrionale Hoffm., qu'on retrouve à Ezery.

Parmi les éboulis du pied: Polypodium vulgare L., $P$. calcareum Sm., et Cystopteris fragilis Bernh.

Sur les rochers ombragés: Phegopteris Dryopteris Fee. Bern., Asplenium Halleri DC., A. Trichomanes L., A. viride Huds., A. Ruta-muraria L., Scolopendrium officinale DG., Pteris aquilina L.

Dans les bois de Sapins: Aspidium aculeatum Doll. Koch., Polystichum spinulosum, DC., P. Filix-mas Roth., Asplenium Filix-fomina Bernh.

Du côté méridional, au-dessus de Cruseilles : Blechnum spicant Roth. 


\section{8}

Enfin, sur les Pitons: Aspidium Lonchytis Sw. et Polypodium rigidum Hoffm., peu abondant.

Nos excursions sont terminées, mais il y aurait encore bien des promenades à faire pour trouver les Mousses, les Lichens et autres cryptogames, dont Salève offre une riche moisson. N'en ayant pas fait une étude spéciale, je ne puis que renvoyer, pour les Lichens, à l'ouvrage de M. J. Nuller, conservateur de l'Ilerbier de M. De Candolle. 


\section{Catalogye des plantes du Iloull-Salève.}

Pour ce Catalogue j’ai consulté :

10 Mon herbier commencé il y a environ quarante ans et dont presque tous les échantillons, concernant Salève, ont élé recueillis par moi, et vérifiés par mon excellent ami Reuter. Il me manque, comme localité, quelques espèces signalés par M. l'Abhé Puget, telles que Barbaren ar cuata Reich., Evonymus latifolius L., Comarum palustre L., Cyclamen hederaefolium Ait., Koch el Dipsacus laciniatus L.

$2^{\circ}$ Les ouvrages classiques de MIM. Gaudin (Flora Ilelvetica), Reuter (Calalogue, Ed. 2), Rapin (Guide, Ed. 2), Godet (Flore du Jura), Grenier (Flore de la Chaine Jurassique, T. I), enfin la notice de II. l'abbé Puget (Bulletin de la Societé Botanique de France, 1866).

J'ai suivi l'ordre du Catalogue de Reuter, d'après lequel est classé mon herbier des environs de Genève.

Cette énumération comprendra toutes les espèces essentiellement montagnardes qui se rencontrent sur les diverses stalions de Salève depuis le pied jusqu'au sommet. 
Quelques-unes descendent quelquefois dans la plaine, par exemple : Thalictrum aquilegifolium L., Ranunculus aconitifolius L., Gentiana verna L., Muianthemum bifolium, DG., Sesleria coerulea Ard., Milium effusum L., Nardus strich L., Equisetum sylvaticum L... etc.

En outre, quelques espèces venant des Alpes, se trouvent par fois dans les sables des îles et dans los bords de l'Arve, ce sont: Viola arenaria DC., Gypsophila repens L., Epilobium Fleischeri IIochs., Saxiliaga aizoides L., Campanula pusilla Hænk., Linaria alpina L., Juncus alpinus Willd... etc.

En revanche, un nombre considérable de plantes campagnardes s'élèvent sur la montagne; les unes, indiflérenies et ubiquistes, s'accomodent aussi bien des bas-fonds de la plaine que des hauteurs du Salève; les autres choisissent les champs et sont cultivées en quelque façon involontairement; d'autres, enfin, parasites de l'homme, le suivent partout où il s'établit et prennent possession du pourtour des habitations et des chalets, telles que: Sisymbrium officinule L., Capsella Bursa-Pastoris Vent., Stellaria media Sm., Malva sylvestris L., M. rotundifoliu L., Senccio vulgaris L., Galeopsis Reichenbachii Reut., Plantago major L., Chenopodium Bonus-Henricus L., Rumex obtusifolius L., Urtica dioica L., U. urens L., Poa annua L.; Festuca pratensis Huds.... etc.

N'ayant pas la prétention de faire une flore complète du Salève, je me suis borné à signaler toutes les plantes essentiellement inontagnardes; quant aux autres, j’ai indiqué celles qui offrent plus d'intérêt. 


\section{DICOTYLEDONES}

\section{THALAMIFLORES.}

Hermancaticere.

Clematis.
SRenomenlacées.

Clématite.

C. vitalbe L. C. des haies, Viorne, Herbe aux Gueux.

Taillis du pied, s'élève assez haut. Juillet, Septembre.

\section{Atragene.}

Atragène.

*A. alpina L. A. des Alpes. Se trouve à deux endroils parmi les éboulements au-dessous des rochers qui dominent Archamp. On m'a dit qu'il en existe dans l'entonnoir de la Petite-Gorge. Mai, Juin.

Thalictrum.

Pigamon.

-T. aquilegifolium L. P. à feuilles d'Ancolie. Bois et pâturages. Mai, Juin.

*T. minus L. II saxatile Gaud. (T. minus L. Rap. God.

T. saxatile Schl. DC. Reut.) P. des rochers. Au pied de la Grande Gorge. Juin, Juillet. Anemone. Anémone.

A. nemorosa L. A. Sylvic. Jusqu'au sommet du PetitSalève. Mars, Avril.

Le signe "indique les plantes montagnardes. 
A. ranunculoides L. $\Lambda$. Renoncule. Sables du bord de l'Arve, près de Veyrier. Mars, Avril.
Hepatica.
Hépatique.

H. triloba DG (Anemone Ilepatica L.). A. Ilépatique.

Broussailles et taillis du pied. Mars, Avril.

Ranunculus. Renoncule.

R. trichophyllos. Chaix. Gr. et G. (R. aquatilis var. pantothrix Gaud.) R. capillaire. Mares du picd. Avril, Juin.

R. paucistamineus Tausch. (R. Drouettii Reut. Rap.

Non Schultz, d'après Grenier.) R. ì étamines peu nombreuses. Mares entre Mornex et Reignier. Avril, Mai.

Observ. Plusieurs anteurs réunissent ces deux espèces qui se ressemblent effectivement beaucoup. Le vrai R. Drouettii Schultz appartient, suivant M. Grenier, à l'Ouest et au midi de la France et ne se trouve pas dans notre dition.

R. divaricalus Schr. R. divariquée. Mare au-dessus de Crevin, rare. Juin, Juillet.

"R. Thore. L. R. Thora. Sommet de la Grande-Gorge, seule localité. Mai, Juillet.

${ }^{*} R$. Aconitifolius L. R. à feuilles d'Aconit. Pâturages humides, bords des ruisseaux, descend quelquefois dans la plaine. Juin, Juillet.

*R. Platanifolius L. R. à feuilles de Platane. Bois de sapins, près des Pitons, vers le sommet. Juin, Juillet.

*R. gracilis. Schl. Reut. (R. montanus Willd. Gren. R. montanus Willd. $\beta$ tenuifo!ius DC, $\beta$ gracilis Rap.) R. grêle. Pàturages du sommez près de la Grande-Gorge et des Pitons. Mai, Juin.

${ }^{-} R$. nemorosus DG. R. des bois. Bois et taillis. Juin, Juillet. 
${ }^{*} R$. lanuginosus. L. R. laineuse. Bois de sapins vers les Pitons, peu commun Juin, Juillet.

Observ. Les $R$. repens L. R. rampante, R. Acris L. R. âcre, $R$. bulbosus L. R. bulbeuse, montent dans les prés et les cultures de la montagne.

\section{Ficaria. Ficaire.}

F. Ranunculoides Mœnch. (Ranunculus Ficaria L.), F. Fausse-Renoncule. Plante ascendante qu'on retrouve dans les pâturages, autour des chalets. Avril, Juin.

\section{Galtha.}

\section{Populage.}

C. palustris L. P. des marais. Depuis la plaine jusqu'autour des mares voisines des chalets. Avril, Juin.

Trollius.

Trolle.

*T. Europaeaus L. T. d'Europe. Pâturages du sommet. Mai, Juin.
Helleborus.
Hellébore.

II. viridis L. H. vert. Verger's de la commune de Pers, entre Reignier et la Roche. Mars, Avril.

H. foctidus L. II. fétide, Pied-de-Griffon. Taillis du "pied. Février, Avril.
Nigella.
Nigelle.

N. arvensis L. N. des champs, Araignée. Plante erratique, champs à Reignier. Juin.
Aquilegia.
Ancolie.

A. vulgaris L. A. commune, Gants. Buissons du pied, s'élève assez haut. Mai, Juin.
Delphinium.
Dauphinelle.

*D. consolida L. D. Pied d'Alouelte des champs. Champs à Monetier, Mornex. Juin, Juillet.
Aconitum.
Aconit.

A. Lycoctonum L. A. Tue-Loup. Taillis du pied. Juin, Juillet. 
Observ. Aconitum Anthora L., indiqué par M. l'abbé Puget, ne croîl pas à Salève, mais sur le Vuache.

\section{Actaea. Actée.}

${ }^{*}$ A. spicata L. A. en épi. Taillis au-dessus de Crevin, de Collonges, d'Archamp. Avril, Mai.

\section{HBerberieleac.}

Berberis.

\section{Berberidées.}

Vineticr.

B. mlgaris L. V. commun, Epine-vinelle. Dans tous les taillis du pied. Mai, Septembre.

\section{Nymphrencene.}

Nymphaea.
Nymphéncées.

Nénuphar.

N. alba L. N. blanc. Marais de Troinex, Bossey. Juin, Juillet.

\section{Honpreveracene.}

Papaver.

\section{Papaveracées.}

Pavot.

P. Argemone L. P. Argémone. Champs de Honetier. Juin, Juillet.

P. dubium L. a Lecoqii Lamot. el $\beta$ collinum Bogen. Bor. P. douteux. Champs arides. Veyrier, Monetier, etc. Mai, Juin.

Obs. P. Rheres L., P. coquelicot, Rose-de-Loup, dans tous les champs de la montagne, de Juin à Juillet. Chelidoninm majus L., Chélidoine Eclaire, au bord des haies, sur les vieux murs des villages de Mai à Juillet.

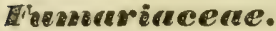

Corydalis.

\section{Fomariacées.}

Corydale.

C. Iuberosa L. (C. cava Gaud.). C. lubéreuse. IIaies et buissons, partout. Mars, Avril.

C. bullosa DG. (C. solida Gaud). C. bulbeuse. Plaine des rocailles, autour de la Pierre-aux-Fées. Mars, Avril. 
Fumaria.

Fumeterre.

F. Chavini Reut. (F. Vaillantii Lois, d'après Rapin), F. de Chavin. Champs de la ferme des Beulets et des Avenières. Juin, Juillet.

Obs. Le F. officinalis L., F. officinale, se trouve dans toutes les cultures de la montagne. Avril, Août.

cracifende.

Barbarea.

\section{Crucifères.}

Barbarée.

B. arcuata Reich., B. arquée. Découverte par MI. l'Abbé Delavay en 1865, près d'Allonzier, entre l'Eglise et le Bureau de la Douane. Mai, Juin.

Turritis.

Tourette.

T. glabra L., T. glabre. Près de Pommier et au vallon des Usses. Mai, Juin.

Arabis.

Arabette.

*A. alpina L., A. des Alpes. Eboulements et rochers, commune. Avril, Juin.

*A. anriculata Lam., A. auriculée. Eboulis du Pas-del'Echelle, carrière entre Veyrier et Crevin, rochers du Coin, peu abondante. Mai, Juin.

*A. saxatilis All., A. des rochers. Rocher's du Coin, rare. Mai, Juin.

*A. hirsuta Scop., A. velue. Prés secs ou tourbeux du pied, vieux murs. Mai, Juin.

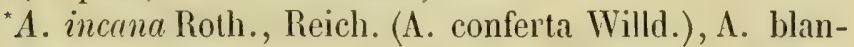
châtre. Rocailles du pied, autour des Carrières. Mai, Juin.

A. sagittata Bertol., DC., A. sagittée. Pied du PetitSalève, au-dessus de Bossey. Mai, Juin.

*A. alpestris Schl., Reich. (A. hirsuta I sessifolia Gaud, A. ciliata $\beta$ hirsuta Koch, $\Lambda$. arcuta $\beta$ hirsula God.), A. alpestre. Pìturages rocailleux du sommet. Juin, Juillet. 


\section{6}

"A. muralis Bert., A. des murs. Rocailles du pied, Pasde-l'Echelle, Coin, Croisette, sur les murs de Bossey. Mai, Juin.

*A. hybrida Reut., A. hybride. Pas-de-l'Echelle, carrières de Veyrier. Mai, Juin.

*A. stricla IInds., A. roide. Eboulis du Pas-de-l'Echelle, Carrières, Coin, Archamp. Mai, Juin.

${ }^{*}$ A. serpyllifolia Vill., A. à feuilles de Serpolet. Fissures des rochers des deux Grands-Pitons. Inin, Juillet.

"A. Turrita L., A. Tourette. Taillis rocailleux du pied. Mai, Juin.

\section{Cardamine. Cardamine.}

C. amma L., C. amère. Ruisseau à Collonges, au Coin, fontaine des Pitons. Mai, Juin.

C. pratensis L., G. des prés. Prairies et bois, s'élève jusque sur les sommets. Avril, Juin.

C. Matthioli Moret., Bert. (G. pratensis y IIayneana Reich.). C. de Matthiole. Marais de Troinex. Avril, Mai.

*C. sylvatica Link, G. des bois. Bois de Sapins, près des Pitons. M. le curé Chavin et M. l'abbé Puget. Mai, Juin.

"C. impatiens L., C. impatiente. Sommet du Pas-del'Echelle, à Monetier vers l'Eglise. Mai, Juin.

Dentaria. Dentaire.

"D. digitata L., D. digitée. Bois du pied, Crevin, Archamp. Avril, Juin.

"D. pinnata Lam., D. pennée. Mêmes localités, plus commune. Avril, Juin.

Sisymbrium.

Sisymbre.

"S. acutangulum DC. (S. autriacum Jacq.), S. d'Autriche. Rochers du Coin, du Pas-de-l'Echelle, Voûtes. Mai, Juin. 
"S. Sophia L., S. saggesse. Voûtes du Petit-Salève, localité unique pour Genève. Mai, Juin.
Conringia.
Conringie.

C. orientalis Andrz. (Erysimum orientale R. Br., Brassica orientalis L.), Chou d'Orient. Erratique et rare, dans les champs de Monetier. M. Berneil. Juin. Juillet.

\section{Lunaria. Lunaire.}

* $L$. rediviva L. L. vivace. Rochers au-dessus de Crevin, du Coin, d'Archamp, de Pommier. Mai, Juillet.
Draba.
Drave.

"D. aizoides L., D. aizoïde. Commune sur tous les rochers vers les sommets. Avril, Juin.

\section{Kernera. Kernère.}

${ }^{*} \boldsymbol{K}$. saxatilis Reich. God. (Cochlearia saxatalis Lam. Myagrum saxalile L.), Cranson des rochers. Eboulis du pied, Pas-de-l'Echelle, Carrières, etc. Mai, Juin.
Camelina.
Caméline.

C. sativa Cr. (Myagrum sativum L.), C. cultivée. Champs de Monetier. Juin, Août.
Thlaspi.
Tabouret.

T. arvense L., T. des champs. Champs de Mornex, de Monetier. Mai, Juin.
Iberis.
Ibéride.

I. amaria L., I. amère. Champs de Veyrier, de Nonetier. Juin, Juillet.

I. pimata Gouan., I. pennée. Champs de Veyrier, de

Monetier, moins commun. Juin, Juillet.

\section{Hutchinsia.}

Hutchinsie.

II. petraea Br. (Lepidium petraeum L.), Passerage des rocailles. Ehoulis du Pied, Pas-de-l'Echelle, etc. Mars, Avril. 
"N. paniculata DG. (Myagrum paniculatum L.), N. paniculée. Champs de Monetier. Juin, Juillet.

\section{Bunias.}

Bunias.

B. Evucago L., B. fausse-Roquette. Champs du mont de Sion, de Gruseilles. Juin, Juillet.

Obs. On trouve dans les champs, les cultures, les prairies, les décombres, au pied des murs et des haies les Cardamine hirsuta L., Cardamine velue, Sisymbrium officinale Scop. (Erysimum officinale L.), Sisymbre officinal, S. Thatiamm Gaud. (Arabis Thaliana L.), S. de Thalius, Alliarin officinalis DC. (Hesperis alliaria L.), Alliaire officinale, Sinapis arvensis L., Moutarde des champs, avec la var. Schkuhriana Reich., Erucastrum obtusangulum Reich. Koch (Sisymbrium obtusangulum DG., Brassica erucastrum Gaud.), Erucastre à angles obtus, Alyssum calycinum L. Alysson calycinal, Erophila verna DG. (Draba verna L.), Drave printanière, avec quelques-unes des espèces de Jordan, Thlapsi perfoliatum L., Tabouret perfolié, Lepidium campestre R. B. (Thl. campestre L.), Tabouret des campagnes, Capsella Bursa-Pastoris Vent. (Thl. Bursa-Pastoris L.), Tabouret Bourseì-Pasteur, Rapistrum rugosum All. (Myagrum rugosum L.), Rapistre ridé, Raphunus raphanistrum L., Radis sauvage, Ravenelle. Toutes plantes de la plaine qui s'élevent sur la montagne et qu'on trouve depuis le début du printemps jusqu'à la fin de l'été.

On cultive en outre : Brassica oleracea L., Chou potager, $B$. rapa L., Chou rude, rave, navelte d'été, et $B$. napus L., Chou navet, navette d'hiver, dans tous les hameaux de Salève. 
mesentucene.

Reseda.
Resedacécs.

Reseda.

R. phytheuma L., R. raiponce. Plante erratique, dans les champs, près de Veyrier. Juin, Août.

R. lutea L., R. jaune. Graviers du Pas-de-l'Echelle. Juin, Août.

\section{Drosercucue.}

Drosera.

\section{Diposépacées.}

Rossolis.

- D. longifolia L. (D. anglica Huds.), R. à feuilles longues. Pelits marais au-dessus de Grevin. Juin, Juillet.

D. olovata M. et K., R. à feuilles ovales. Marais de Bossey. Juin, Aộut.
Parnassia.
Parnassie.

$P$. palustris L., P. des narais. Prés marécageux. Juin, Juillet.

\section{Polygatene.}

Polygala.

\section{Polygalées.}

Polygale.

$P$. amara L. Koch, P. amer. Prairies humides de la montagne. Juin, Juillet.

" $P$. alpestris Reich. (P. amara L. $\beta$ alpestris Koch, $\beta$ alpina Gaud.), P. des Alpes. Pâturage des Pitons. MM. Chavin et Rapin. Juin, Juillet.

${ }^{*} P$. Chamaebuxus L., P. faux-Buis. Crête du Petit-Salève, plaine des Rocailles. Mars, Juin.

Obs. On trouve les $P$. comosa Schk., P. chevelu, $P$. vulgaris L., P. commun, partout au pied de la montagne, et le $P$. austriaca Crantz. (P. amara L. $\gamma$ austriaca Koch, P. uliginosa Reich.), P. d'Autriche, dans les marais de Troinex et Bossey, de Mai à Juillet.

\section{Cistimeare.}

Helianthemum.

\section{Cistinées.}

Helianthème.

H. Fumana Mill. DG., H. Fumana. Eboulis du pied,

Pas-de-l'Echelle, Carrières. Mai, Août. 
"H. canum Dun. Gaud. (H. CElandium Wahl. $\beta$ canum Rap.) H. blanchàtre. Rocailles du pied, de Veyrier à Archamp. Mai, Juin.

H. vulgare Grertn. $\beta$ hirsutum Koch, H. commun, var. velue. Eboulis du pied. Mai, Juillet.

$\gamma$ grandiflorum Koch, var. à grandes fleurs. Pàturages rocailleux de la montagne. Juin, Juillet.

\section{Violcsiene.}

Viola. violarices.

Violette.

V. abortiva Jord., V. avortée: Bois à Mornex. Mars, Avril.

V. alba Bess. (V. scolophylla Jord. Rap. Reut. et V. virescens Jord. Reut.), V. blanche. Taillis et haies du pied de la montagne. Mars. Avril.

V. multicanlis Jord., V. multicanle. IIaies à Veyrier. Mars, Avril.

V. mirabilis L., V. admirable. Broussailles entre Veyrier el Crevin. Avril, Mai.

V. syluatica Fries. (V. sylvestris Lam.), V. des bois. Bois du pied, s'élève assez haut. Avril, Mai.

V. Riviniana Reich. (V. sylvestris $\beta$ Riviniana Koch), V. de Rivin. Bois du pied. Avril, Maii.

V. Canina L. (V. pumila Ging.), V. canine. Pàturages du sommet, avec la var. $\beta$ minor Reut, dans les endroits humides. Mai, Juin.

" $V$. nemoralis Jord. (V. canina L. var. nemoralis Rap.), V. canine var. des bois. Prairies humides vers le Grand-Piton. Mai, Juin.

V. pumilu Vill., Gr. et G. (V. pratensis M. et K.), V. naine. Petites mares au-dessus de Crevin. Mai, Juin.

V. Sagoti Jord. (V. tricolor L. var.), V. de Sagot. Champs des Treize-Arbres, Juin, Juillet. 
Obs. Les V. odorata L. V. odorante, V. hirta L. V. hérissée, V. permixta Jord (V. hirta L. var.) se trouvent partout dans les prés et au bord des haies.

$V$. odorata L. var. albiflora se trouve derrière Salève. Les $V$. agrestis Jord., Pensée des champs et $V$. segetalis Jord., Pensée des moissons, variétés du V.tricolor L., Pensée tricolore, se rencontrent dans tous les champs de la montagne.

\section{Caryophyileae.}

Silene.

\section{Caryoplinyllées.}

Silène.

S. mutuns L., S. penché. Rocailles du pied. Mai, Juillet. Lychnis. Lychnide.

* $L$. diuma Sibt. (L. alpestris Hopp. Gaud.), L. diurne. Bois au-dessus d'Archamp. Nai, Juillet.

L. flos-Cuculli L., L. fleur-de-Coucou. S'élève sur les prairies humides de la montagne. Mai, Juin.

Agrostemma. Agrostemme.

A. Githago L. (Lychnis Githago Lam.), A. Nielle. Champs de la montagne, Monetier, etc. Juin, Juillet.

Saponaria.

Saponaire.

S. ocymoides L., S. basilic. Rocailles du pied. Mai, Juin. Gypsophila.

Gypsophile.

G. vaccaria Sm. (Saponaria vaccaria $\mathrm{L}$. G. des vaches. Champs à Mornex, Monetier. Juin, Juillet.

\section{Dianthus. Eillet.}

D. prolifer L., 0. prolifëre. Rocailles du pied. Juin, Juillet.

${ }^{*}$ D. Carthusianorum L., 0. des Chartreux. Pàturages sees du sommet. Juin, Juillet.

"I. mipicole Jord. (D. sylvestris Gaud.), 0. des rochers. Rocailles dı̊ pied. Juin, Août.

\section{Sagina. Sagine.}

S. procumbens L., S. couchée. Champs sablonneux près de Cruseilles. Juin, Août. 
"S. Linnaci Presl. (Spergula saginoides L.), S. de Linnée. Prairies humides vers les Pitons. Juin, Juillet. Alsine.

Alsine.

A. Icnuifolia Crantz $\beta$ viscidula Gaud. (Arenaria viscidula Thuil.), A. à feuilles menues, variété visqueuse. Eboulis, carrières, champs maigres à Veyrier, assez rare. Mai, Juin.

A. laxa Jord. (A. tenuifolia var.), A. effilée. Hêmes localités, peu commune. Mai, Juin.

A. Mybrida Jord. (Arenaria tenuifolia L. var. hybrida Vill.), A. hybride. Sables d'Arve, sous Veyrier. Mai, Juin.

"A. fasciculata M. et K.(Arenaria fasciculata Jacq.Gouan), A. fasciculée. Pas-de-l'Echelle, carrières, ctc. Juin, Juillet.
Mochringia.
Møhringée.

'M. muscosa L., M. mousse'. Rochers frais du pied. Mai, Juillet.

"M. trinervia Clairv. (Arenaria trinervia L.), M. trinerviẻe. Bois de sapins, au-dessus d'Archamp. Mai, Juillet.

Arenaria.

Sabline.

*A. grandiflora L., S. à grandes fleurs. Rochers du PetitSalève, éboulements vers la Grande-Gorge, au-dessus de Grevin, au Coin. Mai, Juin.
Stellaria.
Stellaire.

* S. nemorum L., S. des forêts. Bois de sapins. Juin, Juillet.

"S. uliginosa Murr. (S. aquatica fl. fr. Larbraca aquatica St. Hill.), S. des marais. Fontaine des Pitons. Juillet, Août.

Cercistium.

Céraiste.

"C. vulgatum L. Gaud. B alpinum Gr. et G., C. vulgaire variété alpine. Pâturages des sommels. Ituin, Juillet. 
"C. arvense L. $\beta$ strictum Reut. (C. strictum L.), C. roide. Pas-de-l'Echelle, éboulis du pied. Mai, Juin.

Obs. On trouve dans les champs de la montagne et dans ceux du pied: Alsine tenuifolia Crantz (Arenaria tenuifolia L.), Alsine à feuilles menues, Arenaria sphœrocarpa Ten. Jord. (A. serpyllifolia Auct.), A. leptoclados Reich. (A. serpyllifolia Auct.), Sabline à feuilles de serpollet, Stellaria media Sm. (Alsine media L.), Stellaire Morgeline, Mouron blanc, surtout autour des chalets, Cerastium brachypetalum Desp., Ceraiste à petales courts, $C$. glutinosum Fries (C. obscurum Koch), C. gluant, C. viscosum L. (C. vulgatum DG., C. glomeratum Thuill.), G. visqueux, C. semidecandrum L., C. à cinq étamines, $C$. vulgalum L. Gaud. (C. triviale Link. Koch), G. commun, Spergula arvensis L., Spargoute des champs, depuis Avril jusqu'à la fin de l'Eté.

\section{Eimeae.}

Linum.

\section{Linées.}

Lin.

L. usitatissimum I., L. cultivé. Gultivé el subspontané dans les champs de Mornex, Monetier, etc., Juin, Juillet.

L. tenuifolium L., L. à feuilles menues. Rocailles du pied. Juin, Août.

L. calluarticum L., L. purgatif. S'élève jusque sur les sommités. Mai, Août.

\section{Braturcene.}

Althaea.

\section{Malvacées.}

Guimauve.

A. hirsuta L., G. velue. Champs arides, près de Veyrier. Juin, Juillet.

Obs. Les Malva sylvestris L., Nauve sauvage, et $M$. rolundifolia L., M. à feuilles rondes, se rencon- 


\section{4}

trent dans les cultures, dans les villages et autour des chalets de la montagne de Juin à Septembre.

Tiliacene.

Tilia.

\section{Tiliacées.}

Tilleul.

T. platyphyllos Scop. (T. grandifolia Ehr.), T. à grandes feuilles. Bois de la montagne, à Monetier. Mai, Juin.

T. intermedia DC., T. à feuilles moyennes Bois du pied. Juin, Juillet.

T. microphylla Vent. (T. parvifolia Ehr., T. sylvestris Desf.), 'T. à petites feuilles. Bois au-dessus d'Archamp, etc. Mai, Juin.

\section{mypeacinese.}

Hypericum.

\section{Hypéricinées.}

Mille-pertuis.

"H. quadrangulum L. (H. dubium Lcers), M. tetragone.

Piturages des Pitons, peu commun. Juillet, Août. H. humifusum L., M. couché. Bois de Veyrier. Août, Septembre.

Var. $\beta$ Liottardi DC. Champs à Cruseilles et aux Avenières. Juillet, Août.

H. montanum L., M. des montagnes. Taillis du pied, Juillet, Août.

H. pulchrum L., M. élégant. Bois de St-Julien et de Viry. Juillet, Août.

Obs. Les H. tetrapterum Fries, M. à quatre ailes. H. perforatum L., M. perforé, herbe aux mille trous, H. hirsutum L, M. velu, s'élevent à une certaine hauteur.

\section{Acenineue.}

Acer.

\section{Acérimées.}

Erable.

A. Pseudo-Platanus L., E. Sycomore. Bois du pied. Mai, Juin.

A. opulifolium L., E. à feuilles d'Obier. Bois du pied et sommet du Pas-de-l'Echelle, à gauche. Avril Mai. 
Obs. L'Acer campestre L., E. champêtre, se trouve dans les taillis du pied et dans les haies de la montagne.

\section{Ampelialere.}

Vitis.

\section{Ampélidées.}

Vigne.

V. vinifera L., V. commune. Subspontanée dans les éboulis du Pied. Juin.

\section{Gerdusincene.}

Geranium.

\section{Géraniacées.}

Géranium.

G. sanguineum L., G. sanguin. Rocaills du pied. Mai, Août.

"G. sylvaticum L., G. des bois. Bois et pàturages des sommités. Juin, Juillet.

G. palustre L., G. des marais, rare, près du Châble. Juin, Juillet.

•G. lucidum L., G. luisant. Pas-de-l'Echelle vers la fontaine, mont Gosse, Voûtes du Grand-Salève. Mai, Juin.

Obs. Les G. pyrenaicum L., G. des Pyrénées, G. molle L., G. mollet, G. pusilum L., G. fluet, $G$. rolundifolium L., G. à feuilles rondes, G. columbinum, L., G. columbin, G. dissectum L., G. disséqué, G. Robertianum L., G. Ilerbe-à-Robert, Érodium triviale Jord. (Geranium cicutarium L. Gaud.), Erodium à feuilles de ciguë, si communs dans la plaine, se retrouvent à la montagne le long des haies et des murs, dans les prairies, les champs et les cultures.

\section{Butsumernecue.}

Impatiens.

\section{Balsaminées.}

Impatiente:

I. noli-tangere L., I., n'y touchez pas, Balsamine sauvage. Rare, dans les cours de l'ancienne Chartreuse de Pommier. Juillet, Août. 
Dxalialeac.

Oxalis.

\section{Dxalidées.}

Oxalide.

O. acelosella L., Oseille, Alleluia, Pain de coucou. Bois du pied, s'éleve assez haut. Avril, Juin.

Obs. L'O. Europaer Jord. (0. stricta Auct.), 0. d'Europe, 0 . droite, se trouve dans les cultures de la montagne. Eté. 


\section{CALYGIFLORES.}

\section{Célnstrimene.}

Evonymus.

\section{Célastriné es.}

Fusain.

E. Europaeus L., F. d'Europe. Bois carré, Taillis du pied. Mai, Juillet.

E. latifolius L., Fusain à larges feuilles. Espèce nourelle pour Salève, découverte par M. l'abbé Delavay, dans les broussailles rocailleuses, au-dessus de l'église d'Allonzier, entre ce village et celui d'Avregny. (Notice et lettre de M. l'abbé Puget.) Mai, Juin.

\section{Beframsuene.}

Rhamnus.

\section{Rhamnées.}

Nerprun.

${ }^{*} R$. alpinus L., N. des Alpes. Taillis du pied. Juin, Juillel. R. catharticus L., N. purgatif. Taillis du pied. Juin, Juillet.

R. frangula L., N. Bourdaine. Taillis du pied. Mai. Juin.

\section{Eégerminosue.}

Genista.

\section{Légumineuses.}

Genêt.

G. Germanica L., G. d'Allemagne. Bois du pied. Mai, Juin.

G. pilosa L.. G. velu. Bois au-dessus de Crevin, rare. Avril, Mai.

\section{Cytisus.}

Cytise.

C. Laburnum L., G. faux-Ebénier, Aubours. Taillis du pied. Mai, Juin. 
Ononis.

Bugrane.

*O. rotundifolia L., B. à feuilles rondes. Voûtes supépéricures du Petil-Salève, éboulis entre Crevin et le Coin. Mai, Juin.

Anthyllis.

Anthyllide.

*A. montana L., A. de montagne. Monticules rocailleux et découverts, entre le Coin et Grevin. Mai, Juin.

Melilotus.

Mélilot.

M. arvensis Walr. (M. officinalis Lam.), M. des champs.

A Monetier dans les champs. Juin. Août.
Trifolium.
Trèfle.

T. mbens L., T. rouge. Taillis rocailleux du pied. Juin, Juillet.

T. ochroleucum L., T. jaunâtre. Prairies sèches du pied et de la montagne. Juin, Juillet.

${ }^{*} T$. alpestre L., T. alpestre. Rocailles du Pas-de-l'Echelle, près de Monetier. Juin, Juillet.

T. medium L., T. intermédiaire. Prairies sèches et monticules du pied. Juillet, Août.

* T. montanum L., T. de montagne. P'rairies de la montagne. Mai, Juillet.

T. aureum Poll. Gr. et G. (T. agrarium Gaud.), T. doré.

Chemin des Chàtaigniers, entre Etrembières el Mornex. Juillet, Août.

Lotus. Lotier.

L. tenuis Kit. (L. corniculatus L., var. tenuifolius Gaud.,

L. tenuifolius Reich.), L. à feuilles ménues. Champs humides près de Veyrier. Juin, Août.
Astragalus.
Astragale.

A. Cicer L., A. Chiche. Au bas de la Croisette et près. de Viry. Juillet, Août.

Coronilla.

Coronille.

G. Emerus L., C. Faux-Séné. Bois du pied. Avril, Mai. ${ }^{*}$ C. vaginalis Lam. (C. minima DG.), C. engainante. Ro- 
cailles du pied, entre Crevin et Archamp. Mai, Juillet.

Vicia.

Vesce.

"V. dumetorum L., V. des buissons. Chemin de la Croi-

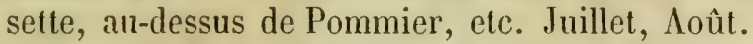

*V. sylvatica L., V. des Bois. Dans les taillis; Monetier, la Croisetle, Archamp, Pommier. Juillet. Août.

V. temuifolia Roth. (Gracca tenuifolia Gr. et G.), V. à feuilles menues. Sur le Petit-Salève, au-dessus de Monetier. Mai, Juin.

Lathyrus.

Gesse.

L. sylvestris L., G. sauvage. Buissons du pied. Juin, Août.

L. hirsutus L., G. velue. Champs à Veyrier. Juin, Juillet.

Orobus.

Orobe.

O. vernus L., 0. printanier. Taillis du pied. Mai, Juin.

O. niger L., O. noir. Taillis du pied. Mai, Juin.

O. unberosus L., 0. tubereux. Broussailles du pied. Avril, Mai.

Obs. Les Genista sagittalis L., Genêt ailé, Anthyllis vulneraria L., Anthyllide vulnéraire, Medicago Lupulina L., Luzcrne Lupuline, Trifolium pratense L., Trèfle de prés, Hippocrepis comosa L., Hippocrepide en ombelle, Lathyrus pratensis L., Gesse des prés, communs dans les prairies, se retrouvent sur tous les pâturages de la montagne. Le Trifolium arvense $\mathrm{L}$., Trèfle des champs, vient en été dans tous les champs.

Les Lotus corniculatus L., Lotier corniculé, et Tetragonolobus siliquosus Roth. (Lotus siliquosus L.), L. siliqueux, s'élèvent dans les prairies humides des sommités.

Les Viciu sepium L., Vesce des haies, et $V$. 
cracca L., V. multiflore, montent dans les buissons jusque près des sommels.

On cultive généralement: Medicago sativa L., Luzerne cultivée, Onobrychis sativa Lam. DC. $(0$. vulgaris Gaud.), Esparcette commune, Faba vilgaris Nœnch. (Vicia Faba L.), Vesce Fève, Vicia sativa L., V. cultivée, Pesette, Pisum sativum L. Pois cultivé, $P$. arvense L., P. des champs, Phaseolus vulgaris L., Ilaricot commun, et P. nanus L.; II. nain. Toutes ces plantes se trouvent en été.

\section{mosucere.}

Prunus.
Rosacées.

Prunier.

P. spinosa L., P. épineux, Epine noire. Ilaies du pied et des villages. Avril, Mai.

$P$. fruticans Weihe (P. spinosa L. var. $\beta$ Koch), P. arborescent. IIaies et taillis du pied. Avril, Mai. Cerasus. Cerisier.

C. avium DG. (Prunus avium L.), C. des oiseaux, Merisier, Margale. Taillis du pied. Avril, Mai.

*C. Mahaleb DC. (P. Mahaleb L.) C. Mahaleb, C. de SainteLucie. TRocailles entre Ftrembières et Mornex, et çá et lả entre Veyrier et le Châble. Avril, Mai.

C. Padus Lois. DG. (P. Padus L.), C. à grappes, Bois joli. IIaies près de Reignier. Mai, Juin.

\section{Spiraea.}

Spirée.

S. Aruncus L., S. Barbe de Chèvre. Taillis du pied. Mai, Juin.

S. Filipendula L., S. Filipendule. Marais de Veyrier, de Troinex, etc. Mai, Juin.
Dryas.
Dryade.

${ }^{*} D$. octopelala L. D. à huit pétales. Au bord du trou de Briffaud, au-dessus de la Groiselle, seule localité. Juin. 
Gerım.

Benoîte.

G. rivale L., B. des ruisseanx. Prairies humides. Juin, Juillet.

G. urbanum L., B. commune. Taillis du pied. Juin, Juillet.

'G. montanum L., B. des montagnes. Pâturages du sommet autour du premier Piton (II. Bernell). Mai, Juillet.

\section{Rubus:}

Ronce.

'R. saxatilis L., R. des rochers. Eboulis du pied. Mai, Juillet.

R. coesius L., R. bleuâtre. Buissons du pied d'où il s'élève jusqu'au sommet. Mai, Juillet.

$\boldsymbol{R}$. dumetorum W. et N., R. des buissons. Dans les haies à Hornex, Monetier, avec la var. a virescens Merc. Juin, Juillet.

${ }^{*} R$. hirtus W. et N., R. hérissée. Pentes du Grand et Petit-Salève, au-dessus de Monetier. Juin, Juillet. ${ }^{*} R$. Guntheri W. et N., R. de Gunther. Mêmes localités. Juin, Juillet.

$R$. discolor W. et N., R. dıscolore, Mûron. Bois et haies du pied de la montagne, avec la var. $\beta$ rusticanus Merc. qui s’élève assez haut. Juin, Juillet.

${ }^{*} R$. collinus DG., R. des collines. Entre Mornex et Monetier, avec la var. Mybridus Merc. Juin, Juillet.

'R . cuneifolius Merc., R. à feuilles en coin. Au-dessus de Mornex. Juin, Juillet.

' $R$. elongatus Merc., R. allongée. Bois au-dessus de Monetier. Juin, Juillet.

" $R$. albidus $\beta$ Saluevae Merc., Ronce blanchâtre de Salève. Monetier. Juin, Juillet.

${ }^{*} R$. Idaeus L., R. frạmboisier. Bois de la montagne, du pied aux sommets. Mai, Juin.

'R. Coesio-Idaens Merc. Les trois formes de cet hybride 
se tronvent autour de la ferme du Beulet au-dessus d'Ar'champ: Nai, Juin.

\section{Fragaria. Fraisier.}

F. vesca L., F. des bois. Dans tous les bois de la montagne. Mai, Juillet.

F. collina Ehrh., F. des collines. Rocailles du pied. Nai, Juin.

\section{Comarum. Comaret.}

C. palustre L., G. des marais. Près du bureau de la douane de la Caille. Nolice de M. l'abbé Puget. Juin, Juillet.

\section{Potentilla. Potentille.}

P. mpestris L., P. des roches. Petit-Salève au-dessus du château de Monelier. Mai, Juin.

$P$. alba L., P. blanche. Sous les Chataigniers entre Etrembières et Mornex. Avril, Mai.

'P. cuulescens L., P. caulescente. Rochers du vallon des Usses, au-dessus des Bains de la Caille. M. le curé Chavin. Juin, Juillet.

${ }^{*} P$. petiolulata Gaud. (P. caulescens $\beta$ petiolnlosa Ser.)

P. petiolulée. Rochers, Pas-de-l'Echelle, an-dessus de Grevin, vallon des Usses. M. A. IIuet. Juin, Juillet.

P. verna L., P. printanière Paturages secs de la montagne. Avril, Mai.

'P. Jurana Reut. (P. alpestris Ilall. fil., P'. Salisburgensis Ilænk., P. aurea Gaud., d'après Rapin),

P. du Jura. P'atturages des J'itons. Mai, Juillet.

*P. aurea L. Koch (P. Ilalleri Ser.), P. dorée. Pâturages des Pitons. Juin, Juillet.

P. Tormentilla Nest. (Tormentilla erecta L.), P. tormentille. liruyères de la montagne. Mai, Juillet.

Rosa. Rosier.

${ }^{*} R$. pimpinellifolia L. * vulgaris God., R. pimprenelle 
commun. Partout depuis les rocailles du pied jusqu'au sommet. Mai, Juillet.

"3 spinosissima Reuter (R. spinosissima L.) près des Treize-Arbres. Juin, Juillet.

${ }^{*} R$. alpino-pimpinellifolia Reut. Cette forme hybride se trouve au bas des rocher's de la Serratula, sur Apchamp. Juin, Juillet.

"R. pimpinellifolio-alpina Rap. Cette forme hybride existe au pied de la Grande-Gorge. Juin. Juillet.

${ }^{*} R$. alpina L. (R. pimpinellifolia Vill., R. glandulosa Bellard, d'après Rap. et Gren.), R. des Alpes. Ians tous les taillis, depuis le pied jusqu'au sommet, avec les trois variétés: $\beta$ pyrenaica Gonan, $\gamma l a$ genaria Vill., ơ aculeala Ser. Mai, Août.

Olss. L'échantillon du $R$. glundulosa Bell., étiqueté de la main de l'auleur et qui existe dans l'Herbier de Candolle, est d'après M. Rapin un $R$. alpina L. Bertoloni avait déjà constaté le même fait (Fl. Ital., V., p. 210). Voir Grenier, Flore Jurassique, T: I, p. 229.

*R. Sabauda Rap., R. de Savoie. Sommet du GrandSalève. Juin, Juillet.

"R. coronalu Crep. (R. Sabauda $\beta$ coronata Rap.), R. couronnée. Taillis et broussailles du pied. GrandeGorge, Groisette, Petit-Salève. Juin, Juillet.

"R. Sulaevensis Rap., R. de Salève. Taillis an-dessus d'Archamp, Croisette, Treize-Arhres. Juin, Août.

'R. spinulifolia Dem., R. à feuilles épineuses. Taillis du pied, au-dessus d'Archamp, au Beulet. Juin, Août.

'R. vestita God., R. velu. Versant du Grand-Salève audessus de Monetier, taillis an-dessus d'Archamp. Juin, Septembre.

'R. montana Chaix. Vill. (R. rubrifolia var. montana 
Ser.), R. des montagnes. Taillis an-dessus d'Ar-. champ. Juin, Septembre.

${ }^{*} R$. rubrifolia Vill., R. à feuilles rouges. Buissons et talus du pied et du versant occidental. GrandeGorge. Juin, Septembre.

${ }^{*} \boldsymbol{R}$. marginuta Wallr. (R. to̊mentosa Sm. $\beta$ marginata Rap.), R. marginé. Taillis au-dessus d'Archamp. Juin, Septembre.

R. mollissima Fries, R. mollet. Rocailles du pied. Mai, Août.

*R. subglobosa Sm. (R. Iomentosa Sm. y subglobosa Rap.), R. subglobuleux. Eloulis rocailleux du Pasde-l'Echelle, au-dessus d'Archamp. Juin, Septembre.

R. tomentosa Sm. R. tomenteux. Taillis du pied. Juin, Septembre.

"R. Reuteri God. (R. monticola Rap. a Reuteri Rap., R. glauca Vill.), R. de Reuter. Taillis au-dessus d'Archamp, chemin de la Croisette, Treize-Arbres. Juin, Septembre.

${ }^{*} R$. monticola Rap. $\varepsilon$ cinereu Rap., R. montagnard. Buissons de la montagne. Juin, Septembre.

${ }^{*} R$. ulpestris Reut. (R. monticola Rap. $\beta$ alpestris Rap.), R. alpestre. Autour des Pitons. Juillet, Septembre.

${ }^{*} R$. Chavini Rap., R. de Chavin. Hybride des R. montana? et $R$. canina. Au bas de la Grande-Gorge et près de la Croiselte. Juin. Septembre.

"R. coriifolia Fries. (R. monticola Rap. $\delta$ frutetorum Rap., R. dumetorum Gr. el G., R. canina var. dumetorum Ser.), R. des buissons. Treize-Arbres, Croisette, au-dessus d'Archamp. Juin, Septembre. "Var. biserrala Reut. Monetier, Treize-Arbres, Croisette. Juin, Septembre. 
R. caninu L. $\beta$ senticosa God., R. commun, Eglantine. Chemin de la Groisette. Juin, Septembre.

y sarmentosa God. Même localité. Juin, Septembre.

$\delta$ collina Gaud. Bois du pied. Juin, Septembre.

'R. spluerica Grem (R. canina L. $\beta$ sphaerica Rap.),

R. sphérique. Grande-Gorge, sentier des TreizeArbres. Juin, Septembre.

" $R$. biserrata Mer. (R. canina L. $\gamma$ biserata Rap.), R. â doubles dentelures. Grand-Salève, au-dessus de Monetier. Juin, Septembre.

R. Andegavensis Bast. (R. canina L. o Andegavensis Rap.), R. d'Anjou. Haies au-dessus du hameau du Coin. Juin, Septembre.

R. mbiginosa L. R. rouillé. Taillis du pied. Juin, Septembre.

var. nemorose Reut. Au-dessus d'Archamp. Juin, Septembre.

R. tomentella Lem. (R. Lomentosa Sm. $\gamma$ dumetorum Gaud.) R. pulsescent. Etrembières, Veyrier, Archamp. Juin, Septembre.

'R. micrantha Sm. (R. rubiginosa a parvifolia God.) R. a petites fleurs. Rocailles au-dessus d'Archamp. Juin, Septembre.

'R. umbellata Leers. (R. comosa Rip. R. tenuiglandulosa Mer.) R. en ombelle. Broussailles du pied, bois de Veyrier. Juin, Septembre.

*R. fotida Bast. R. fétide. Bois de Barioz, près de lá Caille. M. le curé Chavin et M. l'abbé Puget. Juin, Septembre.

R. sepium Thuil. (R. rubiginosa var. sepium Gaud, R. Klukii Bes., R. inodora Fries, d'après Rapin). R. des haies. Buissons du pied. Juin, Seplembre. $\delta$ pubescens Reut. (R. stylosa var. glandulosa Ser. d'après Rapin), près de Crevin. Juin, Septembre. 
R. arvensis Iluds. R. des champs. Bois du pied. Juin, Septembre.

R. Gullicu L. (R. Austriaca Crantz, R. pumila Jacq. d'après Grenier, Fl. Jurass.) R. de France, R. de Provins. Haies, près de Veyrier. Juin, Aoùt.

R. Gallico-umbellata Ratp. (R. consanguinea Gren. FI. Jur.) Forme hybride trouvée par II. Rapin dans une haie près du bois de Veyrier. Juin, Aoùt.

R. hybrida Sch. (R. Gallico-arvensis) R. hybride. Haies près de Veyrier. Juin, Août.

R. Gallico-canina Reut. (R. psilophylla Rau. Deségl. d'après Gren. Fl. Jur.) Ilaies près de Veyrier. Juin, Août.

\section{Alchemilla. Alchémille.}

A. vulyaris L. A. commune, Pied-de-lion. Paturages de la montagne. Juin, Juillet.

"A. hybride Willd. Bor. (A. vulgaris var. y Gaud. A. pubescens Koch. A. Iruncata Tausch.) A. hybride. Paturages secs des sommités. Juin, Juillet.

-A. alpina L. A. des Alpes, Argentine. Sommet de la Grande-Gorge, Pitons, etc. Juillet, Août.

Sanquisorba. Sanquisorbe.

S. officinalis L. (S. serolina Jord. Reut. S. montana Jord. Reut.) S. officinale, Grande-Pimprenelle. Depuis la plaine jusque sur les pâturages humides de la montagne. Juin, Septembre.
Cratuegus.
Aubépine.

C. oxyacontha L. (Mespylus oxyacantha Di. Crataegus monogyna dacq. Koch). A. commune, Epine blanche. Buissons du pied, haies de Mornex, Monetier. Mai, Juin.

C. oxyacanthoides Thuil. (C. oxyacantha. Koch. Gaud. Godet, Rapin). A. à deux noyaux. Mêmes localités, plus précoce. Avril, Juin. 
Obs. Les auleur's ne sont pas d'accord au sujet de ces deux espèces : les uns les réunissent et ne les distinguent pas même comme variétés; les autres intervertissent les noms, d'autres en font trois espèces, en sorte qu'il est assez difficile de s'y reconnaitre. M. Reuter fait observer que le $C$. oxyacanthoides Thuill. est plus précoce, qu'il a des fleurs plus grandes, une odeur désagréable, des fruits plus gros, d'un rouge plus foncé et à deux noyaux. Le C. oxyacantha L. (C. monogyna Jacq.) est bien la plante qui se trouve dans l'herbier de Linné. Il est plus tardif, il a des fleurs odorantes, à un seul style, des fruits plas petits, à un seul noyau, des pédoncules velus el les feuilles sont divisées en lobes profonds, aigus et dentés en scie.

\section{Cotoneaster.}

Cotonnier.

C C. ulgaris LindI. (Mespilus coloneaster L.) C. commun. Rocailles du pied. Mai, Juin.

"C. tomentosa Lindl. (Mespilus tomentosa Gaud.) C. tomenteux. Rochers au-dessus de Crevin, plus rare. Mai, Juin.

Amelanchier. Amélanchier.

*A. vulgaris Mœnch. (Mespilus Amelanchicr L. Crataegus Amelanchier Gaud.) A. commun. Rocailles du pied. Mai, Juin.

\section{Mespilus.}

Néflier.

'M. Germanica L. N. commun. Trouvé près du sommel du Petit-Salève, au-dessus du village de Nonetier, par II. Rapin, qui l'a récolté en fleurs au mois de Juin. Il parait bien spontané. Juin, Septembre.

Sorbus.

Sorbier.

S. domestica L. (Pyrus Sorbus Gaertn.) S. domestique. Bois de Veyrier, près du Chàble. Mai, Août. 
*S. Aucupuria L. (Pyrus Aucuparia Crantz. Koch.) S des Oiseleurs. Bois du pied. Mai, Juillet.

*S. hybrida L. S. hybride. Bois au-dessus d'Archamp. Juin, Août.

"S. Aria Crantz. (Crataegus Aria L. Pyrus Aria DC.) S. Alizier. Bois du pied. Mai, Aoùt.

*S. Scandica Fries, Koch. (Crataegus Aria a Scandica L. Pyrus intermedia DG., Sorbus intermedia Reich.) S. de Scandinavie. Taillis au-dessus de Crevin, d'Archamp, de Pommier. Mai, Août.

S. torminalis Crantz. Koch. (Grataegus torminalis L. Gaud. Pyrus torminalis DC.) S. à feuilles anguleuses. Bois du pied. Mai, Août.
Pyrus.
Poirier.

P. communis L. P. commun. Taillis du pied. Avril, Août.
Malus.
Pommier.

M. communis Poir. (Pyrus malus L.). P. doux. Bois du pied. Avril, Août.

M. acerba Mer. (Pyrus acerba DC.). P. acerbe. Bois de Veyrier. Avril, Août.

\section{Onagravieae.}

Epilobium.
Onagrariées.

Epilobe.

"E. spicutum Lam. (E. angustifolium L. Gaud.). E. en épi. Bois de la montagne. Juillet, Septembre.

E. montanum L. E. des montagnes. Taillis du pied. Juin, Juillet.

E. collinum Gmel. (E. montanum L. $\beta$ collinum Koch.) E. des collines. A Cruseilles. Juillet, Août. E. Lamyi Schultz. Bor. (E. virgatum Koch. non Fries. E. tetragonum L. $\beta$ Lamyi Rapin.) E. de Lamy. Clairières du bois de Veyrier. Juillet, Octobre. 
Isnardia. Isnardie.

1. pulustris L. I. des marais. Endroits marécageux du bois de Viry. M. Chavin. Aoùt, Septembre.
Circaea.
Circée.

C. Lutetiana L. C. commune. Collonges. Juillet, Aoùt.

\section{Lythrarieare.}

Lythrum.

\section{Lythrariées.}

\section{Salicaire.}

L. hyssopifolia L. S. à feuilles d'Hysope. Clairières du bois de Veyrier. Août, Septembre.

Peplis.

Péplide.

P. Portula L. P. pourpier. Bois de Veyrier et de Viry. II. Ghavin. Juillet, Aoùi.

\section{Trumariseineac.}

Myricaria.

\section{Tamariscinées.}

Myricaire.

M. Germanica Desv. (Tamarix Germanica L. Gaud.) M. d'Allemagne. Bords de l'Arve, sous Veyrier. Mai, Octobre.

\section{Parmychiene.}

Scleranthus.

\section{Paronychiécs.}

Gnavelle.

'S. peremnis L. G. vivace. Couches de sable sur' le revers méridional, près de Cruscilles. Juin, Juillet. S. biennis Reut. G. bisannuelle. Sur le sommet près des Pitons. Juin, Juillet.

S. verticillatus Reich. (S. Delorti Billot.) G. verticillée. Sommel du Pelit-Salève, M. Rapin. Mai, Juin.

Crassulacere.

Sedum.

\section{Crasgulacées.} Orpin.

S. purpurascens Koch (S. Telephium L. $\beta$ purpureum

L.) 0. purpurin. A Collonges. Août, Septembre. -S. maximum Koch (S. Telephium d et \& L.) 0. à larges feuilles, Reprise. Vallon des Usses, près de la Caille. Août, Septembre. 
'S. villosum L. 0. velu. Autour d'une mare creuséc dans le sable siliceux, entre la Croisette et les Pitons. Juillet, Août.

S. ulbum 1. O. blanc. Rocailles du pied, s'élève assez haut. Juin, Juillet.

S. dasyphyllum L. 0 . à feuilles épaisses. Rocher's du . pied et de la région moyenne. Juin, Juillet.

S. acre L. O. acre. Eboulis du pied. Juin, Juillet.

$S$. sexungulare L. 0 . à six angles. Hêmes localités. Juin, Juillet.

S. reflexum L. 0 . refléchi, avec la var. $\beta$ gluncum Koch. Rocailles du pied. Juin, Juillet:

$S$. unopelulum DC. 0 . i pétales dressés. Rochers à Crevin, au Coin. Juin, Juillet.

\section{Crossuluriene.}

Ribes.

\section{Growsulariées.}

Groselier.

R. Une-crispe L. (R. Grossularia L. $\beta$ pubesens Koch.) G. épineux. Haies du pied et des villages de la monlagne. Avril, Juin.

*R. alpinum L. G. des Alpes. Broussailles du pied. Avril, Juin.

\section{Suxifragere.}

Saxifraga.

\section{Saxifragées.}

Saxifrage.

"S. uizoon Jacq. (S. colyledon var. ₹ L.) S. aizoon. Sur tous tes rochers de la monlagne. Mai, Juillet.

'S. aizoides L. S. faux aizoon. Ruisseau au-dessus de Crevin. Juillet, Septembre.

S. tridactylites L. S. Iridactyle. Eboulis at rocailles. Mars, Avril.

S. granulata L. S. granulée. Entre Veyrier et Crevin. Mai.

'S. rolumdifolia L. S. à feuilles rondes. Bois de la monlagne. Mai, dtin. 
Chrysosplenium.

Dorine.

"C. alternifolinm L. D. a feuilles alternes. Fontaine des Pitons. Avril, Mai.

\section{Umbelliferce.}

Sanicula.

\section{Jumbellifèrew.}

Sanicle.

S. Europaea L. S. d'Europe. Bois du pierl. Juin, Juillet.

Astrantiu.

Astrance.

*A. mujor L. A. majeure. Pàturages. Juin, Aoùt.

Trinia.

Trinie.

'T. vulguris DC. (Pimpinella dioica L. Trinia glabertima Dub. T. Henningii Gaud.) T. commune. Eboulements et rochers du pied et de la région moyenne. Mai, Juillet.

Carum.

Carvi.

*C. Carvi L. C. officinal. Cumin des prés. Pàturages de la montagne. Juin, Juillet.

C. Bullocustanum Koch. (Bunium Bulbocastanum L.) C. noix-de-terre. Champs à Collonges Juin, Juillet. Pimpinella.

Boncage.

'P. magna L. avec la var. $\beta$ rosea Koch. B. majeur, Grande Pimprenelle. Eboulis herbeux du pied et pâturages des sommités. Juin, Août.

$P$. saxifraga L., B. saxifrage, petite Pimprenelle. Rocailles du pied. Juin, Septembre.

Enanthe.

Enanthe.

E. Lachenulis Gm. (E. peucedanifolia Gaud.), (E. de Lachenal. Marais de Bosscy. Juillet, Août.

Foniculum.

Fenouil.

F. officinale All. (Anethum foniculum L.) F. officinal. Bords des vignes à Etrembières. Juillet, Septembre.

Seseli.

Séséli.

S. bienne Grantz. Gaud. (S. annuum L., S. coloratum 
Ehrh.), S. bisannuel. Eboulis du pied, au Coin, au-dessus de Crevin. Juillet, Septembre.

Athamantha.

Athamanthe.

'A. Crelensis L., A. de Crête. Rochers au-dessus d'Archamp, de Crevin, au Coin. Juin, Juillet.

Selinum.

Sélin.

S. carvifolia L. (Mylinum carvifolia Gaud.), S. a feuilles de Carvi. Clairières humides du bois de Veyrier. Juillet, Septembre.

\section{Peucedanum. Peucedanum.}

-P. Cervuria Koch (Cervaria glauca Gaud., Selinum Cervaria DC.), P. des Cerfs. Sur le Petil-Salève. Juillet, Septembre.

Heracleum. Berle.

'H. montunum Schl. Gaud. (H. Panacea L., II. asperum Koch), B. des montagnes. Eboulements au-dessus d'Archamp. Juillet, Seplembre.

Laserpitium.

Laser:

'L. Siler L. Siler. Rochers du pied. Juin, Septembre.

'L. lutifolium L., L. à grandes leuilles. Rochers du pied el de la région moyenne. Juillet, Septembre.

L. Pruthenicum L., L. de Prusse. Bois de Veyrier. Juillet, Septembre.

Orlaya.

Orlaya.

O. grendiflora Hoffm. (Caucalis grandiflora L.), 0. a grandrs fleurs. Champ it Troinex, Veyrier, Nonelier. Juin, Juillet.

Cancalis.

Cancalide.

C. Dancoides L., C. à feuilles de carotle. Champs à Veyrier, Monetier. Juin, Juillel.

Chaerophyllum.

Cerfenil.

* O. Ciculuria Vill. (C. hirsutum Reut.), C. Cicutaire. Au-dessus du Chàble. Juin, Juillet. 
Scandix.

Scandix.

S. Pecten-Veneris L., Peigne de Vénus. Champs de Monetier. Mai, Juin.

Anthriscus.

Anthrisque.

"A. abortivus Jord. (A. Cicutaria Dub., A. alpestris W. et G. d'après Reut., A. sylvestris var. alpestris Koch, d'après Rap.), A. avorté. Montée des Pitons, au-dessus de Pommier. Juillet, Septembre.

A. vulgaris Pers. (Scandix Anthriscus L.), A. commun. Voûtes du Petit-Salève. Mai, Juillet.
Myrrhis.
Myrrhe.

"M. odorata Scop. (Scandix odorata L.), M. aromatique, Cerfeuil musqué. Pâturages sur le Grand-Piton. Juin, Septembre.

Obs. Aqopodium podagraria L., Egopode podagraire, Herbe-aux-goutteux Silans pratensis Bess. Gaud. (Peucedanum Silaus L.). Silaus des prés, Brise-pierre. Angelica sylvestris L., Angélique sauvage. Heracleum sphondylium L., Berle Brancursine. Anthriscus sylvestris Hoffm. (Chaerophyllum sylvestre L. DC.), Anthrisque sauvage, Persil d'àne, remontent dans les prairies et les pâturages de la montagne. Tout l'été.

AEthusa cynapium L., Ethuse vénéneuse, petile Ciguë. Daucus carotta L., Carotte commune. Anthriscus cerefolium Hoffm. (Scandix cerefolium L), Cerfeuil, se rencontrent dans les champs et les cultures des villages. Tout l'élé.

\section{Aratiacene.}

Hedera.

\section{Arallacées.}

Lierre.

H. Helix L., L. grimpant. Arbres, murs, rochers, jusqu'au sommet. Tout l'été. 
Cormene.

Cornus.

Cornées.

Cornoullier.

C. sunguinea L., L. sanguin. Taillis du pied. Mai, Juin.

\section{Hormuthacene.}

Viscum.

\section{Loranthacées.}

Gui.

V. album L. G. commun. Parasite sur les arbres. Mai, Juin.

\section{Caprifolincere.}

Adoxa.

\section{Caprifollacées.}

Adoxe.

A. Moschatellina L., A. moscatéline, Ilerbe-all-muse. Bord des haies. Mars, Avril.

Sambucus.

Sureau.

S. Ebulus L., S. Yèble. Vryrier, Collonges. Juillet, Août.

S. migrt L., Sureau commun. Haies et taillis du pied. Mai, Juin.

${ }^{*}$ S. racemosa L., S. à grappes. Taillis du pied et de la région moyenne. Avril, Juin.

Viburnum. Viorne.

V. Opulus L., V, Obier. Taillis du pied. Mai, Juin.

V. Lentana L. V. Mancienne. Taillis du pied. Mai, Juin.

Lonicera. Chèvre-feuille.

L. Periclymenum L. C. sauvage. Taillis du pied. Juin, Juillet.

L. Xylosteum L., C. des haies. Taillis du pied. Avril, Mai.

'L. vigra L., C. noir. Taillis au-dessus d'Archamp, de Pommier. Mai, Juin.

"L. alpigenu L., C. des Alpes. Bois rocailleux du pied et de la région moyenne. Avril, Juin. 
Hubiacene.

Galium.

\section{Rubiacées.}

Gaillet.

G. boreale L. G. boreal. Glairières humides du bois de Veyrier. Juin, Juillet.

G. glaucum L. (Asperula galioides DC.) G. glauque.

Prairies artificielles à Veyrier. Mai, Juin.

"G. sylvaticum L. G. des bois. Taillis de la montagne. Juillet, Août.

G. sylvestre Poll. G. sauvage. Taillis du pied. Juin, Juillet.

var. a laeve Thuil. (G. commutatum Jord. G. montanum Vill. Gr. et G.)

var. $\gamma$ Boccone All., mêmes lieux. Juin, Juillet.

G. uliginosum L. G. aquatique. Marais de Bossey. Iuillet, Août.

*G. Spurium L., G. bâtard. Champs de la montagne. Juin, Juilleț.

var. $\beta$ vaillantii Gr. el G. Champs des Treize-Arbres. Juillet, Août.

*var. y tenerum Gr. el G: (G. tenerum Schl., Gand.)

Voûtes du Pètit-Salève. Mai, Juin.

G. Iricorne With. (G. à trois cornes). Champs à Monetier. Juin, Juillet.
Asperula.
Aspérule.

${ }^{*} A$. odorata L., A. odorante. Hépalique des bois. Bois de la montagne. Mai, Juin.

A. arvensis L., A. des champs. Cultures à Veyrier, Monetier, etc. Mai, Juin.

A. cynanchica L., A. des sables. Ilerbe à l'esquinancie. Eboulis du pied. Juin, Juillet.

Sherardia.

Rubéole.

S. arvensis L., R. des champs. Cultures de la montagne. Juin, Août: 
Vuleriamear.

Valeriana.
Valérlanées.

"Valériane.

"V. tripteris L., V. à trois ailes. Eboulements du pied. Mai, Juillet.

${ }^{*} \boldsymbol{V}$. montana L., V. des montagnes. Mlêmes localités, Grevin, le Coin, Archamp. Mai, Juillet.

$V$. dioica L., V. dioique. Ruisseaux à Bossey, Collonges, etc. Avril, Mai.

Valerianella.

Mâche.

*V. Morisonii DC., a leiocarpa God. (Fedia dentata, Gaud.) M. de Morison. Champs à Monelier. Mai, Juin.

V. auricula DC. (Fedia auricula Gaud.) M. oreillette. Champs à Monetier, Mornex, etc. Mai. Juin.

V. olitoria Møench. (Fedia olitoria Gaud.) M. commune, Doucelte, Rampon. Cultures de la montagne. Avril, Mai.

\section{Dipsacene.}

Dipsacus.

\section{Dipsacées.}

Cardère.

D. laciniatus L. C. découpée. Trouvé en 1865, par M. l'abbé Delavay, an bord de la rivière des Usses, au-dessous de Cernex. Notice de M. l'abbé Puget. Juillet, Août.

Cephalaria.

Céphalaire.

C. pilosa Gr. et G. (D. pilosus L.) C. poilue. Près de Veyrier. Juillet, Septembre

\section{Knautia.}

Knautie.

K. sylvatica Dub. (Scabiosa sylvatica L.), K. des bois. Bois du pied. Juillet, Septembre.

Scabiosa.

Scabieuse.

S. succisa L. S. mors-du-diable. Prairies et bois du pied. Juillet, Septembre. 
Compositue.

Adenostyles.
Composées.

Adénostyle.

A. albifrons Reich. (Cacalia albifrons L., C. Pelasiles Lam.), A. velu. Bois de la montagne. Juillet, Août.

A. alpina B. et F. Koch. (Cacalia alpina L. Gaud.), A. des Alpes. Ehoulis au bas de la Grande-Gorge. Juillet, Août.

Homogyne.

Homogyne.

'H. alpina Cass. Koch. (Tussilago alpina L.), H. des Alpes. Pâturages élevés. Juin, Août.

\section{Petasites. Pétasite.}

P. officinalis Mœnch. (P. vulgaris Desf. Tussilago Petasites L.), P. officinal. Bords de l'Arve, près du pont de Belle-Combe. Avril, Nai.

P. Reuteriana Jord. (P. officinalis Mœnch. var. Reuteriana), P. de Reuter. Verger de Bossey. Avril, Mai.
Tussilago.
Tussilage.

T. Farfara L. T. taconet, Pas d'àne. Dans lous les terrains humides el argilleux. Février, Mars.

Solidago. Verge d'or.

"S. alpestris W. K. (S. virga aurea var. alpestris Koch.)

V. des Alpes. Pâturages rocailleux. Juillel, Août.

Erigeron. Vergerelle.

* E. ulpinus L. V. des Alpes. Sur le Grand-Piton. N. Rapin. Juillet, Août.

Aster.

Aster.

A. Amellus L. A. Amellus. Eil de Christ. Hocailles du pied. Août, Septembre.

Bellidiastrum. Bellidiastre.

B. Michelii Cass. (Margarila Bellidiastrum Gaud. Doronicum Bellidiastrum L.) B. de Micheli. Endroits 
frais du pied el de la région moyenne, jusqu'au sommet. Mai, Juillet.

Pulicaria.

Pulicaire.

P. vulgaris Gærın. (Inula pulicaria L.) P. commune. Fossés â Veyricr. Août. Septembre.

Doronicum.

Doronic.

* D. Pardalianches L. D. à feuilles en coeur, Mort-auxPanthères. Hlaie au-dessus de Veyrier, taillis vers la Fontaine de Jules-César, pentes du Grand-Saleve au-dessus de Nonetier, sur Archamp. Mai, Juin.

Arnica.

Arnica.

- A. monlanu L. A. de montagne, Tabac des Vosges. Pàturages de l'extrémité méridionale des Pitons, au-dessus de Cruseilles, rare. Juin, Juillet.

Senecio.

Séneçon.

'S. viscosus L. S. visqueux. Hocailles du Pas-de-l'Échelle. Juin, Juillel.

-S. Jacobaea L. S. Jacobée. Mont Gosse, au bas de la Croisette. Juin, Juillet.

* S. flosculosus Jord. (S. Jicubaea L. var. llosculosa Ciaud.) S. flosculeux. Rorailles du Pas-de-l'Échelle. Juin, Juillet.

"S. Fuchsii Gmel. (S. alpestris Gaud, S. nemorensis L., S. Sarracenicus Gr. et G.) S. de Fuchsius. Bois au-dessus d'Archamp, rare. Juillet, Août.

Leucanihemum. Leucanthème.

- L. corymbosum Gr et G. (Chrysanthemum corymbosum L. Gaud.) L. en corymbe. Bois rocailleux du pied. Juin, Juillet.

\section{Matricaria.}

Matricaire.

M. Chamomilla L. M. Camonilie, Petite Camomille. Cha mpsà Reignier. Mai, Juillet. 
Anthemis.

Anthemis.

A. Cotula L. A. fétide, Camomille puante. Champs ì Crevin. Juillet, Septembre.

Gnaphalium. Gnaphale.

* G. sylvaticum L. (G. rectum Sm.) G. des bois. Bruyères et pâturages de la montagne. Aoùt, Septembre.

Antennaria. Antennaire.

-A. dioica. Gærtn. (Gn. dioicum L. Gaud.) A. dioique, Pied-de-char. Pâturages des sommités. Mai, Juillel.

Filago.

Cotonnière.

F. spathulata Presl. (F. Germanica $\beta$ pyramidata Gaud., F. Jussiaei Cos. el Germ.) C. à feuilles spathulées. Champs du pied el de la montagne. Juillet, Septembre.

$F$. Germanica L. Gaud. (F. lutescens Jord. et F. canescens Jord.) C. d'Allemagne. Champs à Veyrier, Monetier, etc. Juillet, Septembre.

F. arvensis L. Gaud. C. des champs. Champs à Veyrier, rare. Juillet, Septembre.

F. Gallica L Gaud. C. de France. Champs à Veyrier, rare. Juillet, Septembre.

F. minimu Fries. (F. montana L. Gaud.) C. naine. Champs sablonneux à Cruseilles. Juillel, Septembre:

Cirsium.

Cirse.

* C. eriophorum Scop. (Carduus eriophorus L.) C. laineux. Pàturages des sommités. Juillet, Septembre. C. acaule All. (C. acaulis L.) C. nain. Pàturages rocailleux du pied et des sommets. Juillet, Septembre. C. arvense Scop. (Serratula arvensis L. Carduus arvensis Willd.) C. des champs, Herbe-aux-Varices. Cultures de la plaine et de la montagne. Juin, Août. 
Carduns.

Chardon.

C. defloratus L. C. terne. Ehoulis et rocailles. Juin, Août.

Centaurea.

Centaurée:

C. Jacea L. C. jacée. Prairies du pied et de la région moyenne. Juin, Juillet.

'C. montana L. C. de montagne. Au-dessus d'Archamp, rare. Juin, Août.

C. Cyanus L. C. bluet, Barbeau. Champs de la plaine et de la montagne. Juin, Août.

"C. Scabiosa L. B petrophila Reut. C. Scabieuse. Rochers du Pas-de-l'tichelle, de la Grande-Gorge. Juin, Juillet.

Serratula.

Serratule.

'S. mudicaulis DC. (Centaurea nudicaulis L.) S. a lige nue. Assises des grandes roches perpendiculaires au-dessus d'Archamp, seule localité; pen abondante. Juin, Juillet.

Lappa.

Bardane.

- $L$. intermedia Reich. B. intermédiaire. Grand-Salève. en montant au Treize-Arbres, depuis Monetier. Juillet, Août.

- L. major Gærtn. B. à grosses tètes. Archamp, aux Avenières, à Présilly. Juillet, Août.

Carlina.

Carline.

C. vulgaris L. C. commune. Eboulis du pied. Juillet, Septembre.

- C. acunlis $\mathrm{L}$. C. naine. Pâturages secs de la montagne. Août, Septembre.

Hypochaeris.

Porcelle.

"H. maculata L. P. tachetée. Pàturages des sommités, descend dans la plaine. Juin, Juillet.

Lentodon.

Liondent.

L. autumnale L. L. d'automne. Prairies du pied et de la région moyenne. Août, Septembre. 
L. hispidum L. (Apargia hispida Gaud.) L. velu. Prés secs de la montagne. Juin, Septembre.

L. hastile L. (A. hastilis Willd. Gaud.) L. commun. Rocailles du pied. Juin, Août.

\section{Scorzonera.. Scorzonère.}

"S. Austriaca Wild. (S. humilis Jacq. DG. non Lin.) S. d'Autriche. Rochers au-rlessus de Crevin et d'Archamp, rare. Mai, Juin.

S. humilis I. (S. plantaginea Schl.) S. it feuilles de plantain. Découverte en 1866 par II. Rapin, autour de petites mares sous la station de l'Atragène. Mai, Juin.

Taraxacum.

Pissenlit.

T. officinale Wigg. Gr. et Godr. $\beta$ laenigatum God. (Taraxacum dens-Leonis Desf. Leontodon Taraxacum L. Taraxacum laevigatum DG.) P. lacinié, Dent-de-lion. Pàturages sees du sommet. Avril, Juin.

T. palustre DG. P. des marais. Endroits marécageux du pied. Avril, Mai.

\section{Lactuca.}

Laitue.

L. dubiu Jord. (L. Scariola Gaud. non Lin. d'après Renter.) L. douteuse. Bord des vignes à Etrembières. Juillet, Août.

L. muralis. Fresen. (Prenanthes muralis L. Chondrilla muralis Lam. Phaenixopus muralis Koch.) L. des murs. Rocher's ombragés du pied. Juin, Septembre.
Prenanthes.
Prénanthe.

P. purpurea L. P. purpurine. Bois du pied. Juin, Août.

Sonchus.

Laitron.

S. arvensis L. L. des champs. Cultures de lit montagne. Juillet, Août. 


\section{Crepis: $\quad$ Crépide.}

C. foetida L., Gaud. (Barkhausia foetida DC.) G. fétide. Près de Veyrier, entre Mornex el Monetier. Iuin, Août.

-C. uureu Cass. , Koch. (Leontodon aureum L. Hieracium aureum Scop.) Crépide dorée. Pîturages du sommet entre la Croisette et les Pitons, rare. Juin, Juillet.

C. biennis L., C. bisanmuelle. Prairies du pied et de lat région moyenne. Mai, Juillet.

Soyera.

Soyère.

'S. puludosa Godr. (Ilieracium paludosmm L. Crepis paludosa Møench.) S. des marais. Bois et prés humides de la montagne. Juin, Juillet.

Hieracium.

Epervière.

H. pilosella L., E. piloselle, Oreille-de-Souris. P'rés secs du pied el de la région moyenne. Mai, Octoble.

H. anricula L. (II. dubium Gaud.) E. auricule. Paturages humides, depuis la plaine jusque sur les sommets. Hai, Octobre.

H. prueallum Vill. (H. fallax. Gaud.) E. élevée. Marais de Troinex. Juin, Juillet.

H. pilosello-praeultum Schult\%. (H. hybridum Gaud.)

E. hybride. Trouvé au pied de la montagne par

M. Muret, rare. Mai, Juin.

II. florentinum All. (H. praealtum Gaud. H. piloselloides Vill.) E. de Florence. Bords de l'Arve vers l'embouchure de la Nenoge. Juillet, Août.

"H. glancum All.. E. glauque. Rochers de la GrandeGorge, du Coin, d'Archamp. Juillet, Août.

H. staticefolium Vill., E, à feuilles de statice. Eboulements du pied, commun. Juin, Août.

'H. flexuosum W. el K., Gaud., DG. (II. glabratum 
Koch., Gr. et G.) E. flexueuse. Rochers de la Grande-Gorge, peu abondant. Juillet, Août.

'H. villosum L., E. velue. Rocher's des Pitons, rare. Juillet, Août.

'H. dentatum Hopp., $\beta$ Suluevense liap. mss. d'après Reuter. E. dentelée. Versant nord du Petit-Piton, découverte par Mll. Rapin et Chavin, rare. Juillet, Août.

'H. amplexicaule L., E. embrassante. Rocher's de la montagne, abondant. Juillet, Août.

'H. pulmonarioides Vill. (II. amplexicaule $\gamma$ pulmonarioides Gaud.) E. pulmonarioide. Rochers des Pitons, de la Girande-Gorge, du vallon de Monetier. Juillet, Août.

'H. Pseudo-Cerinthe Koch. (II. cerinthoides Vill., II. amplexicaule \& Pseudo-Cerinthe Gaud.) E. de Gaudin. Rochers des Pitons et entre la Grande et la Petite-Gorge, rare. M. Bernett. Juillet, Aoûl.

'H. Ligusticum Fries. (II. amplexicaule L. ל̧ aureun Gaud.) E. de Ligurie. Rochers du Coin, des Pitons, du vallon de Nonetier, etc. Juin, Juillet.

'H. lanatum Vill., E. laineuse. Rocher's au-dessus d'Arcbamp, arec la Serratule, rare. Juin, Juillet.

'H. undryaloides Vill., E. fausse-andryale. Rochers du Pctit-Salève, aut-dessus de Mlonetier, Voùtes du Grand-Salève, rocher's du Fier, l'are. Juin, Juillel. H. murorum L., E. des murs. Bois du pied. Nai, Aoùl. $H$. nemorense ford. (II. murorum L. $\alpha$ nemorense Rap.) E. des forêts. Bois du pied. Nai, Juillet. H. praecox Schultz (H. murorum L. y praecox Rap.) Bois de Crevin. Mai, Juillet.

'H. ccesium Fries. (11. murorum L. ò cusium hap.) E. bleuâtre. Bois du pied. Mai, Juillet.

*H. Jacquini Vill. E. de Jacquin. Rochers du Coin, de 
la Grande-Gorge, du Pas-de-l'Echelle, du PetilSalève. Juin, Juillet.

- H. elalum Fries. (II. prenanthoides II Juramun Gaud.) E. elancée. Bois du sommet, vers les Pitons. Juillet, Août.

"H. melenotrichum lieul. (II. Sibaudum L. ò melanotrichum Rap.) Bois du Grand-Salève, au-dessus du chalet de la Traversaz. (Reuter.) Taillis au-dessus de Gruseilles (Chavin). Rare. C'est probablement une forme hybride entre le II. elatum Fr. el le II. Sabaudum L. Août, Septembre.

II. Iridentutum Fries. (II. rigidun IIatm. Rap.) E. a trois dents. Près de Cruseilles, rare. (II. Chavin). Juillet, Août.

H. boreale Fries (II. sahaudum Gaud. Rap. II Sylvestre T'ausch.) E. boreale. Taillis du pied.

y curvidens Gr. el Godr. Au-dessus de Gruseilles (II. Chavin).

o) virgultorum Gr. et Godr. Bois de Viry. (II. Chavin.)

z dumosum Gr. al G. Bois de Viry (M. Chavin). Aoùt, Septembre.

II. vugum Jord. (H. boreale var. ragum Gr. et G. H. Sabaudum L. \& vagum Rap.) E. vague. Près du Chàble. (II. Chavin.) Aoùt, Septembre.

H. umbellatum L. E. en ombelle. Taillis du pied. Aoùt, Septembre.

Observ. On trouve dans toutes le's prairies: Bellis perennis $\mathrm{L}$. Piquerette vivace, petite marguerite, depuis Avril en Septembre. Leucunthemumvulyare. Lam. (Chrysanthemum Leucanthemum L.) Grande marguerite, de Hai à Octobre. Achilleu millefolium L. Achillée mille-feuilles, herbe au charpentier, depuis Juin à Septembre. Dans toutes les 
cultures et aulour des chalets: Senecio vulgaris I. Senecon commun, presque toute l'année.

\section{Campunentuceue. Campannlacées.}

Jasione.

Jasione.

I. montunu L. J. des montagnes. Chemin des Chàtaigniers, entre Mornex et Etrembières. Juin, Aoùt.
Plyteuma.
Raiponce.

P. spicuta L. flore coeruleo. R. en épi. Petil-Salève, Veyrier. Mai, Juin.

Specularia.

Spéculaire.

S. Speculum A. DC. (Prismatocarpus Speculum L'Her.

DG.) S. Niroir-de-Vénus. Champs à Vonetier. Juin, Juillet.

Campanula.

Campanule.

C. glomerata L. C. agglonérée. Pàturages rocailleux du pied. Juin, Juillet.

C. aggregata N. et B. Gaud. Ci. aggrégée. Bois de Veyrier, taillis du pied. Juin, Juillet.

C. Cervicaria L. C. Cervicaire. Bois de Veyrier, rare. Juin, Juillet.

C. Trachelium L. C. gantelée. Gants de Notre-Dante.

Taillis du pied. Juillet, Aoùt.

$\beta$ dasycarpu Gren. (C. urticaefolia Schm. Gaud.) Taillis du pied. Juillet, Aoùt.

C. rupunculoides L. C. fausse-raiponce. Rocailles du pied. Juin, Juillet.

"C. rhomboidalis L. C. rhomboidale. Pàturages du sommet. Grande-Gorge, entre la Croisette et les Pitons. Juillet, Août.

-C. subramulosu Jord. (C. pusilla Gaud.) G. naine. Rochers et éboulis du pied. Juillet, Août.

C. Rapunculus L. G. Raiponce. Champs de la montagne. Juin, Juillet. 
C. putula L. C. étalée. Taillis du pied. A Pommier. Juillet, Août.

C. persicifoliu L. C. à leuilles de pêcher. Taillis du pied. Juin, Juillet.

Obs. On trouve sur les murs et sur les rocher's $C$. rotundifolia L. C. a feuilles rondes. Iuin, Septembre.

\section{Vrecinciene.}

Vaccinium.
Vacciniées.

Airelle.

"V. Myrtillus L. A. Myrtille, Ambroche, Ambresaille. Bois et pâturages. Mai, Juillet.

-V. Vitis-Idaea L. A. ponctuće. Derrière le Piton du Milieu. Juin, Juillet. 


\section{COROLLIFLORES.}

\section{Evicinere.}

Arctostaphylos.

\section{Wicinces.}

Arbousier.

- A officinalis IT. el G. (Arbutus Uva-UrsiL.) A. officinal, Busserole, Raisin d'Ours. Éboulis et rocailles du pied. Avril, Juin.

Calluna.

Callune.

C. vulgaris Sal. C. (Erica vulgaris L.) C. commune, Bruyère. Pàturages arides. Juillet, Septembre.

pyrofrecue.

Pyrola.
Pyrolacées.

Pyrole.

- $P$. rotundifolia L. P. a feuilles rondes. Bois du pied et de la région moyenne. Juin, Juillet.

- P. media Sw. P. intermédiaire. Éboulements au-dessus d'Archarnp, localité de l'Atragène, rare. Juin, Juillet.

- P. minor L. P. mineure. Bois de sapins, vers les Pitons. Juillet, Aoùt.

- P. chlorantha Sw. P. rerdàtre. Bois de Barioz, près de la Caille, rare. Il. l'abbé Puget. Juin, Juillet.

+ $P$. secunda L. P. unilatérale. Bois du pied et de la région moyenne. Juillet, Août.

Troveotropecte.

Monotropa.
Monotapées.

Monotrope.

II. Hypopitys L. Honotrope suce-pin. 
a ylabra God. (Hypopitys glabra DC.)

$\beta$ hirsuta God. (H. multiflora Scop. DC.) Bois de sapins, rare. Juill t, Août.

\section{primetecene.}

Primula.

\section{Primulacées.}

Primevère.

-P. officinalis Jacq. (P. veris et officinalis L.) P. officinale, coucou. Prairies du pied et de la région moyenne. Mars, Avril.

P. suaveolens Bertol. (P. officinalis Jacq. $\beta$ suaveolens Rap.) P. odorante. Buissons et rocailles dupied, ne se trouve pas dans la plaine. Mars, Avril.

P. variabilis Goup. (P. acauli-officinalis et P. officinali-acaulis Muret.) P. variable. Dans les vergers de la plaine, remonte jusqu'au sommet du PetitSalève. Mlars, Avril.

$P$. elatior Jacq. (P. veris $\beta$ elatior L.) P. élevée. Bois du pied, s'élève assez haut. Avril, Juin.

P. grandiflora Lam. (P. veris y acaulis L. P. acaulis Jacq.) P. a grandes fleurs, Olive. Prairies du pied et de la région moyenne. Mars, Avril.

- P Auricula L. P. Auricule, Oreille d'Ours. Vallon des Usses, rochers de la rive gauche, au-dessus des Bains de la Caille. Mai, Juin.

$P$. farinosa L. P. farineuse. Marais de Troinex, ou il a été introduit autrefois. Mai.

\section{Cyclamen.}

Cyclamen.

* C. Europaeun L. C. d'Europe, Pain de pourceau. Rocailles du pied, Petit-Salève, etc. Juillet, Octobre.

C. hederaefolium Ail. non Ten. C. is feuilles de lierre Découvert par M. l'abbé Delavay, en 1866 , dans le vallon des Usses, an pied des rocher's qui dominent les bains de la Caille. (Notice de M. l'abbé Puget dans le Bulletin de la Soc. bot. de France.) Août, Octobre. 


\section{Lysimachia. Lysimaque.}

" L. nemorum L. L. des bois. Forêts de sapins près des Pitons. Juin, Août.

Centunculus.

Centenille.

C. minimus L. C. naine. Champs argilleux près de Veyrier. Août, Octobre.

Obs. On trouve, dans toutes les cultures de la montagne, Anagallis cornlea Schreb. Mouron blen et A. phonicea de. M. rouge, tout l'été.

\section{Aquifolincene.}

Ilex.

\section{Aquifoliacées}

Houx.

I. Aquifolium L. II. commun. Rocailles du pied. Mai Juin.

oleinene.

Ligustrum.
Dleinées.

Troëne.

L. vulgare L. T. commun, Fresillon. Taillis du pied. Mai, Juin.

Fraxinus.

Frêne.

F. excelsior L. F. commun. Bois du pied. Avril, Mai.

Aselepindene.

Vincetoxicum.
Asclepiadées.

Dompte-venin.

V. officinale Mœnch. (Asclepias vincetoxicum L.) I). officinal. Eboulis du pied. Juin, Juillet. Vinca.

Pervenche.

V. minor L. P. commune, Petite-Pervenche. Taillis. du pied, commune sur le Petit-Salève. Avril, Mai.

Gentianene.

Menianthes.
Gentianées.

Ménianthe.

M. Irifoliata L. M. à trois feuilles, Trèfle de marais. Marais du pied, Troinex, Bossey. Mai, Juin. Chlora.

Chlore.

C. serotina Reich. (C. perfoliata L. $\beta$ pusilla Gaud.) C. tardive. Entre Gaillard et Etrembières. Septembre, Octobre. 
Gentiuna.

Gentiane.

* G. lutea L. G. jaune, Grandegentiane. Pâturages. Juin, Juillet.

G. cruciata L. G. croisette. Rocailles du pied. Juin, Juillet.

*f. verna L. G. printannière. Pâturages du sommet, descend vers Archamp el Pommier. Mars, Juin.

* G. campestris L.G. champêtre. Pâturages élevés. Août, Septembre.

G. Germanica Wild. (G. amarella Pol. Gaud). G. d'Allemagne. Endroits secs el argilleux du pied. Septembré, Octobre.

G. ciliala I., G. ciliée. Endroits humides el argilleux, chemin des Chàlaigniers. Septembre, Uclobre.

Erithraea.

Erythrée.

E. Cenfaurium Pers. (Gentiana Centaurium L., Chironia Centaurium Schm. DC.), E. Centaurée, petile Centaurée. Bruyères el prairies humides du pied. Juillet, Août.

\section{monrmginere.}

Anchusa.

\section{Borraginécs.}

Buglosse.

A. Italica Rel\%., C. d'llalie. Champs de Monetier. Juin, Juillet.
Pulmonaria.
Pulmonaire.

P. officinalis L., P. ofticinale. Taillis près de Veyrier. Avril, Mai.

P. tuberosa Schr. Gr. et G. (P. angustifolia Gaud.), P. tuberculeuse. Bois du pied. Mars, Avril.
Myosotis.
Myosote.

"M. syluatica Ehrh. Gaud. (M. perennis $\beta$ sylvatica DC.), M. de forêts. Bois au-dessus do Collonges et près des Pitons. Mai, Juin.

Echinospermum. Echinosperme. 
E. Lappula Lehm. (Myosotis Lappula L.), E. Bardane. Eboulis et carrières du pied. Mai, Juillet.
Asperugo.
Rapette.

"A. procumbens L., R. couchée. Voûtes du Petit-Salève, seule localité. Mai, Juin.

Cynoglossum.

Cynoglosse.

C. officinale L., C. officinal, Langue de chien. Rocailles du pied, rare. Mai, Juin.

"C. montanum L., C. de montagne. Au pied des grandes roches qui dominent Archamp, au-dessus de Pommier, perl abondant. Juin, Juillet.

\section{solnmene.}

Atropa.

\section{Solanées.}

Atropa.

A. Belladona L., A. Belladone, Belle-dame. Pied du Petit-Salève et près des ruines du convent de Pommier, peu commune. Juin, Août.

\section{Orabanchene.}

Phelipaea.

\section{Orabanehées.}

Phélipée.

P. ramosa C.-A. Mey. (Orobanche ramosa L.), P. rameuse. Parasite sur les racines du chanvre. Troinex; Mornex, Monetier. Août, Septembre.
Orobanche.
Orobanche.

O. Galii Dub. (0. caryophyllea Gaud.), 0. du Gaillet. Pàturages du pied et de la région moyenne. Mai, Juillet.

O. Tencrii Schultz. Reut., 0. de la Germandrée. Eboulis du pied, au-dessus de Veyrier. Juin, Juillet.

*O. Scabioscie Kı ch. Rent., O. de la Scabiense. Eboulements de la Grande-Gorge, rare. Juillet, Septembre.

"O. Laserpiti-Sileris Rap. Reul., 0. du Laserpitium Siler. Eboulements au-dessus de Grevin, rare. Juillet, Août. 
0. Cervariae Suard Gr. et G. (0. brachysepala Schulız. Reut.), 0. du Cervaria. Petit-Salève, au-dessus de Monetier. Juin, Juillet.

\section{Lathrnea.}

Lathrée.

L. squamaria L., L. écailleuse. Au pied des Noyers, entre Crevin et Bossey. Avril, Mai.

\section{verbascene.}

Verbascum.

\section{Verbascées.}

Molène.

V. Thapsus L. (V. Schraderi Meyer. Koch.), M. Bouillon-blanc, Bonhomme. Lieux incultes, clairières, çà el là. Juillet, Août.

'V. nigrum L., M. noire. Monetier'. Juillet, Aoùt.

\section{Scrophoulariacene. Serophulariacés.}

Scrophularie.

S. Balbisii Horn. Koch. (S. aquatica Gr. et G. non L.), S. de Balbis. Moullin d'Aiguebelle près d'Etrentbières, peu répandu. Juin, Juillet. Linaria.

Linaire.

L. Cymbalarin Nill. (Anthirinun Cymbalaria L.) Naturalisé sur les vieux murs de Mornex. Mai, Août. 'L. alpina Mlill. (A. alpinum L.), L. des Alpes. Sables d'Arve, erratique. Mai, toût.

Obs. On trouve pendant l'été st l'automne dans les cultures de la montagne L. spuria Mill. (A. spurium L.), L. bàtarde, L. Elatine Mill. (A. Elatine L.), L. Elatine, L. minor Desf. (A. minus L.), L. naine.

Digitalis.

Digitale.

"I). lutea L. (D. parviflora Lam.), D. à petites fleurs. Ruissons du pied, Pas-de-l'Echelle, au-dessus de Crevin, d'Archamp. Juin, Juillet.

"D. grandiflora Lam., D. à grandes fleurs. Buissons du pied, moins commune que la précédente. Juillet; Août. 
Erinus.

Erine.

${ }^{*}$ E. alpinus L., E. des Alpes. Pas-de-l'Echelle, carrières, éboulis du pied. Mai, Juillet.

\section{Veronica.}

Véronique.

V. Teucrium L., V. Germandrée. Monticules dn pied et pâturages du sommet. Juin, Juillet.

* $V$. urticaefolia L., V. à feuilles d'ortie. Taillis dn pied. Mai, Juin.

"V. montana L., V. de montagne. Bois au-dessus ile. Pommier et près des Pitons. Mai, Juin.

"V. fruticolosa L., V. fruticuleuse. Sur les Pitons, rare. Juin, Juillet.

V. triphyllos L., V. trilobée. Champs à Monetier, peu commune. Avril, Mai.

V. Buxbaumii Ten. (V. filiformis DC.), V. de Buxbaum. Gultures à Veyrier. Avril, Juin.

Obs. On trouve dans les prés du pied et de la montagne: V. serpyllifolia L., $V$. à feuilles de Serpolet. Nai, Juin.

Dans les champs et cultures: V. arvensis L., V. des champs, V. didyma Ten. Gr. cl G. (V. polita fries), V. luisante, $V$. hederaefolia L., V. it feuilles de Lierre, d'Avril à Septembre.

\section{ERoineattucene.}

Melampyrum.

\section{Mhinantacées.}

Mélampyre.

M. pratense L., M. des prés. Bois du pied. Juin, Juillet.

"M. sylvalicum L., M. des bois. Bois de sapins. Juillel, Août.

\section{Pedicularis. Pédiculaire.}

P. palustris L., P. des marais. Herbe-aux-Poux. Marais du pied. Juin, Juillet.

*P. Iuberosa L., P. Lubereuse. Patturages entre la Croi- 
sette et les Pitons, seule localité, rare. MMI. Rapin et Chavin. Juillet.

\section{Rhinanthus. Rhinanthe.}

R. minor Ehrh. (R. Christa-Galli $\alpha$ L.), R. à petites leuilles, Grête de coq. Prairies de la montagne. Juin, Juillet.

R. major Ehrh. Koch. (R. Crista-Galli $\beta$ L), R. ì grandes fleurs. Prairies du pied. Juin, Juillet.

${ }^{*}$ R. Alectorolophus Poll. Koch. (R. Christa-Galli $\gamma$ L., R. hirsutus Lam.), R. velu. Champs ì Bossey, Grevin, Monetier, Treize-Arbres. Juin, Juillet.

${ }^{*} R$. anyustifolius Gm., R. it feuilles étroites. Pàturages des Pitons. Juillet, Août.

\section{Odontites.}

Odontite.

O. rubre Gr. et G. (Fuphrasia verna Bell., F. odonlites Koch., Odontites verna Reich.), O. rouge. Champs a Monetier, Mornex. Juin, Juillel.

Euphrasia. Euphraise.

E. montana Jord. (E. officinalis L. $\beta$ montana Rap.), E. de montagne. Marais de Bossey. Mai, Juin.

E. compestris Jord. (E. officinalis L. $\gamma$ campestris Riap.), E. champêtre. Piturages et bruyères. Septembre, Octobre.

*E. cricelorum Jord. Rent., E. des Bruyères. Pied du Petit-Salève, Coin, Chalet des Avenières. Septembre Octobre.

${ }^{*} \boldsymbol{E}$. uliginosa Ducom. E. des marais Endroit marégacageux, au-dessus du Coin. Juillet, Août.

${ }^{*}$ E. Sollisburyensis Funk. (E. alpina DG. Gaud.), E. de Salzhourg. Eboulis du pied. Pas-de-l'Echelle, Carrières. Aoûl, Septembre.

${ }^{2}$ E. cupraea Jord. (E. Salisburgensis $\beta$ r.upraea Rap.), E. cuivrée. Ehoulis du pied. Août, Septembre. 
Lrebintre.

Mentha.
Labiées.

Menthe.

M. rotundifolia L., $M$. ì feuilles rondes. Mornex. Juillet, Août.

M. syluestris L., M. sauvage. Ruisseaux du pied. Juillet, Septembre.

M. candicans Grantz. (M. viridis L. var. canescens $\mathrm{Gr}$. et G.), M. blanchâtre. Ruisseaux de la montagne. Juillet, Septembre.

M. viridis L., M. verte. Collonges. Juillet, Aoùt.

Salvia. Sauge.

S. glutinosa L., S. glutineuse. Buissons dı pied. Archamp, Collonges. Juillet, Septembre.

S. pratensis L., S. des prés. Prairies du pied et de la région moyenne. Mai, Juillet.

Origanum.

Origan. .

O. vulgare L., O. commun. Taillis rocailleux du pied. Juillet, Septembre.

Thymus.

Thym.

T. Serpyllum L., T. Serpolet. Pelouses sèches du pied et de la région moyenne. Mai, Septembre.

Calamintha.

Calament.

*C. alpinu Lam. Gaud. (Thymus alpinus L.), C. des Alpes. Eboulements el rocher's du pied et des sommets, aux Pitons. Juillet, Août.

C. ascendens Jord. (Thymus Calmintha Auct, d'après Pituler, Calamintha ofticinalis Benth., C. menthaefolia Ilosl., d'après Rapin), C. ascendent. Entre Veyrier et Grevin. Août, Septembre.

C. nepeloides Jord. (C. nepeta Gaud.), C. faux-nepeta. Veyriel el Archamp.

Clinopodium.

Clinopode.

C. mlyare L., C. commun. Taillis dı pied. Juillet, Septembre. 


\section{Glechoma.}

Glechome.

G. hederacen L., Gr. commun, Lierre terrestre. Haies du pied et de la montagne. Avril, Mai.

\section{Melittis.}

Mélitte.

M. Melissophyllum L., M. à feuilles de Mélisse. Taillis du pied. Mai, Juin.

\section{Galeopsis.}

Galeopsis.

${ }^{*}$ G. Reichenbachii Reut. (G. "tetrahit L. y Reichenbachii Rap.), G. de Reichenbach. Chatet derriere les Pitons. Juillet, Août.

"G. macex Jord. (G. tetrahil L. $\beta$ praecox Rap.). Champs à Nonetier. Juin, Juillet.

"G. intermedia Vill. (G. Ladanum L. II latifolia Gaud.),

G. intermediaire. Abondant aux Treize-Arbres, dans lêt champs. Juin, Juillet.

"G. angustifolia Ehrh. (G. Ladanum Auct. non L., d'après Reuter). Rocailles et champs. Juillet, Septembre.

\section{Galeobdolon. Galéobdolon.}

G. Luleum Huds., G. jaune, Orlie jaune. Tailtis du Pied. Mai, Juin.
Stachys.
Epiaire.

*S. alpina L., E. des Alpes. Bois au-dessus d'Archamp. Juillet, Aoû?.

S. arvensis L., E. des champs. Cultures ì Veyrier. Juin, Août.

S. rectu L., E. dressée, crapaudine. Rocailles du pied. Juin, Octobre.
Betonica.
Bétoine.

B. officinalis L., B. officinale. Prés rocailleux dı pierl. Juin, Juillet.
Brunella.
Brunelle.

B. laciniatu Lam. Gand. (B. alba Poll.), B. découpée. Rocailles du pied. Juin, Inillet. 
B. Irendiflore Jacq. Monch. (B. vulgaris $\beta$ grandiflora L.). B. a grandes fleurs. Lieux arides du pied. Juillet, Octobre.

Teucrium. Germandrée.

T. scorodonia L., G. des bois. Taillis du pied. Juillel, Août.

T. Chamuedrys L., G. petit-Chêne. Hocailles, s'élève jusque ver's les sommets. Juillet, Août.

${ }^{*}$ T. montanum L., G. des montagnes, Thym blanc. Riocailles du pied. Juillet, Août.

Obs. On trouve Lamium maculatum L., Lamier lacheté, L. purpureum L. . L. pourpre, L. amplexicaule L., L. embrassant, Ajuga reptens L., Bugle rampante, A. Genevensis L., B. de Genève, le long des haies, dans les prairies et cultures de la montagne depuis Nars à Septembre.

\section{Lentibeduriene. Rentibalariées.}

Pinguicula.

Grassette.

P. vulgaris L., C. commune. Marais du pied. Troinex, Collonge, Archamp. Mai, Juin.

"P. alpina L., G. des Alpes. Marais du mont de Sion, Clairière humide au-dessus d'Archamp, avant d'arriver à la localité de l'Atragène; nouvelle station découverte par M. Bernett. Juin, Juillet.

Utricularia. Utriculaire.

U. vulgaris L., U. commune. Etang du bois de Grevin. Juillet, Septembre.

$U$. minor L., U. naine. Marais de Troinex el de Bossey. Juillet, Septembre.

\section{crobulariene.}

Globularia.

\section{Globnlariées.}

Globulaire.

${ }^{*}$ G. cordifolia L., G. à feuilles en coeur. Rocailles du pied, de Veyrier à Archamp. Mai, Juin. 
G. vulyuris L., G. commune. Mèmes localités. Mai, Juin.

*G. nudicuulis L., G. à lige nue. Trouvé deux lois parl II. Ducommun, all-dessus d'Archamp, à rechercher. Mai, Juin.

\section{plantrginere.}

Plantago.

\section{Plantaginées.}

Plantain.

*P. alpinu L., P. des Alpes. Sur les Pitons, rare. Juin, Juillet.

P. serpentina Vill. (P. integralis Gaud., P. Wulfenii Koch), P. serpentant. Prairies argilleuses an-dessus d'Archamp, vers le Chàble. Juillel, Octobre.

'P. cynops L., P. frutescent. Petil-Saleve, entre Hornex et Monetier. Mai, Octobre.

Obs. On rencontre dans les cultures et les paturages, surtout au-dessus des chalets: P. major L., P. a grandes fenilles, P. des oiseaux, P. media L., P. moyen et P. lanceolata L., P. lanceolé. 


\section{MONOCHLAMYDÉES.}

\section{Ansurumtncene.}

Polycnemum.

\section{Amarantacées.}

Polycnème.

P. arvense L., P’. des champs. Gravière de Veyricr. Juillet, Septembre.

P. mujus A. Bi. (P. arvense $\alpha$ majus Rap.), P. majeur. Gravièıe de Veyrier. Juillet, Septembre.

\section{Chemoporlere.}

Chenopodium.

\section{Chénopodées.}

Ansérine.

C. Bomus-Henricus L., A. Bon-Henri, Epinard sauvage. Autour des chalets. Juillet, Septembre.

Blitum.

Blite.

B. virgatum L., B. effilée. Subspontanée dans des décombres à Collonges, erratique el rare. Juillet, Septembre.

Ohs. On trouve dans les villages de la montagne, soil le long des murs, soit autour des fumiers, soit dans les cultures: C. hybridum L., A. hybride, C. murale L., A. des murs, G. album L., A. blanche, avec les var. $\alpha$ spicigerum $\beta$ cimigerum $\gamma$ concatenutum, $C$. polyspermum L., A. polysperme avec les var. $\alpha$ acutifolium $\beta$ polyspermum, $C$. vuluctria L., A. fédite, C. glancum L., A. glauque, 
Atriplex palula L. Arroche élalée. Elé el atutomne.

On cultive en outre: Spinaciu inermis Monch., Epinat'd sans épines, E. de IIollande, S. spinosu Manch, E. épineux, E. d'hiver. Betu vulyuris L., Bette commune, et Atriplex hortensis L., Arroche des jardins, Bonne-Dame.

\section{Polygonere.}

Rumex.

\section{Polygonées.}

Patience.

R. oblusifolius L., P. a feuilles obtuses. Dans les villages et autour des chatels. Juillet, Septembre.

R. conglomeralus Ilurr., P. aggglomérée. Dans les villages et autour des chalets. Juillet, Septembre.

R. acelosella L., P. petite oseille, Surelle. Monetirr, Mornex. Mai, Juin.

*R. arifolius All., P. à feuilles de Gonel. Trouvé par N. Rapin dans un bois de sapins, en se dirigeant vers la Croiselte, depuis le pied des grandes rorhes verticales au-dessus d'Archamp, seule localité. Juillet.

\section{Polygonum. Renouée.}

P. lapathifolium L. $\beta$ incunum heut., R. à feuilles de Patience. Parties cullivées des marais de Troinex. Juillet, Septembre.

P. Fagopyrum L., R. Sarasin, Blé noir. Gultivé dans les champs de la montagne. Juillet, Septembre. (Ohs. On trouve dans les villages et dans les pitturatges: Rumex pulcher L., Patience ou Oseille pindurée, $R$. crispus L., I'. crèpue et $R$. acetosa L., P. oseille, Oseille des prés.

\section{rhymelere.}

Daphne.
Thymélées.

Daphné.

D. Mezereum L., D. Bois-genlil. Taillis du pied. Mar's, Juin. 
D. Latreolu L., D. Lilureole. Taillis du pied. PetitSálève. Février, Mars.

D. alpina L., D. des Alpes. Eboulements el fissures des rochers entre Veyrier el Archamp. Nai, Juin.

Obs. Le D. Cneorum L., D. Cameléc. indiqué à Sitlive par la Flore frinçaise, ne se trouve qu'all Jura sur la pente nord du Marchairu.

\section{Sundulucene.}

Thesium.

\section{Santalacécs.}

Thésion.

T. pralense Ehrh., T. des prés. Piturages du pied et de la région moyenne. Juin, Juillet.

"T. alpinum L., T. des Mlpes. Paturages pierreux de la montagne. Juin, Juillet.

\section{Eterguene.}

Hippophae.

\section{Eleagraées.}

Argoussier.

H. rhemnoides L., A. laux-nerprum. Eboulis au bas de la Grande-Gorge, au-dessus d'Ar'champ, plus ahondant au bord de l'Arve. Avril, Mai.

\section{Anistorochieuc.}

Asarum.

\section{Aristolochiees.}

Asaret.

A. Europaeum L., A. d'Europe. Taillis du piel. Mars, Avril.

\section{Eepporbiacene. Euphorbiacés. Euphorbia. Euphorbe.}

$\boldsymbol{E}$. dulcis L., E. doux. Bois du pied. Avril, Mai.

E. Cyparissias L., E. Cyprès. S'élève jusqu'au sommet. Mai, Juin.

E. amygdaloides L. (E. sylvatica Jacq.), E. à feuilles d'Amandier. Bois du pied. Mai, Juin.

Buxus.

Buis.

B. sempervirens L., B. vert. Au-dessus de Veyrier, plaine des rocailles. Mars, Avril. 
Obs. On trouve dans les cultures et atu pied des haies de la montagne: E. Helioscopic L., E. Réveillematin, E. plalyphyllos L., E. à larges feuilles, E. exigua L., E. fluette, E. falcata L., E. en faux, E. Peplus L., E. Peplus, Omblette, E. stricta L., E. dressée, Mercurialis annua L., Mercuriale annuelle, $\boldsymbol{M}$. perennis L., M. vivace, depuis le printemps jusqu'en automne.

\section{Erticene.}

Parietaria.

\section{Urticées.}

Pariétaire.

P. erecta M. el K. (P. officinalis L.), P. dressée, P. officinale, Casse-pierre. Rochers ombragés du pied. Juin, Septembre.
Cannabis.
Chanvre.

C. sativa L., C. cultivé. Champs des villages. Juin, Septembre.
Urtica.
Ortie.

U. dioica L., 0. dioïque. Autour des chalets. Juin, Aoùt.

U. urens L., 0. brûlante. Autour des chalets. Juillet, Août.

Juglandere.

Juglans.
Juglandées.

Noyer.

J. regia L. N. commun. Mornex, etc. Mai, Aoùt.

Cupuliferae.

Fagus.

\section{cupulifères.}

Hêtre.

F. sylvatica L. H. des forèts, Fayard. Bois du pied et du sommet. Mai, Juin.

Castanea.

Châtaignier.

C. vulguris Lam. (Fagus Castanea L.), C. commun. Mornex. Juin, Juillet.
Quercus.
Chêne.

Q. pubescens Willd. C. pubescent. Bois du pied. Avril, Mlai. 
Q. peduncululu Ehrh. (Q. racemosa Lam.) C. à fruits pédunculés. Mlèmes localités. Mai, Avril.

Q. sessiliflor Sun. C. à fleurs sessiles, Rouvre. Nèmes localités, plus commun. Mars, Avril.

Corylus.

Coudrier.

C. Avellanu L. C. Noisetier. Taillis du pied el la région moyenne. Février, Avril.

Carpinus.

Charme.

G. Betulus L. C. commun. Taillis du pied Avril, Mai.

Utanueene.

Ulmus.
Ulmacées.

Orme.

"U. montana Sm. Orme des monlagnes. Bois du pied et de la région moyenne. Mars, Mai.

Salicinene.

Salix.
Salicinées.

Saule.

S. alba L. S. blanc, Osier blanc. Bords de l'Arve, sous Veyrier. Avril, Mai.

S. umygdalinu L. (S. triandra L.) S. à feuilles d'Amandier, avec les deux variétés a discolor God. et $\beta$ concolor God. Bords de l'Arve, sous Veyrier. Avril, Mai.

S. purpurea L. S. pourpre, Osier rouge. Taillis du pied. Mars, Avril.

S. Pontederana Willd. Schl. Gaud. (S. purpureo-cinerea Rap., S. purpureo-daphnoides Chav. Mss.)

S. de Pontedera. Cette forme hybride se trouve au bois de Veyrier et au bas de la Grande-Gorge. Mars, Mai.

S. Daphnoides Vill. (S. praecox Hopp.) S. faux-Daphnés. Bords de l'Arve, sous Veyrier. Mars, Avril. S. Seringeanc Gaud. (S. lanceolata Ser., S. Smithiana $\beta$ obscura Gr. el G., S. incano-capraea Chav. Mss.) S. de Seringe. Cette forme hybride se trouve au 
bas de la Grande-Gorge (Rapin) et près des bains de la Gaille (Ghavin). Mars, Avril.

S. incunc Schr. (S. lavandulaelolia Lap., S. riparia Willd.) S. à feuilles cotonneuses. Bords de l'Arve. Avril.

S. nigricans Fries. S. noircissant.

a leiocarpa God. (S. nigricans Sm. S. phylicifolia Wahl). Bord de l'Arve, sous Veyrier. Mar's, Avril. $\beta$ eriocurpe God. (S. nigricans Wahl). Près de la fontaine du Pas-de-l'Echelle. Mars, Avril.

S. cinerea L. (S. acuminata IIoflm.) S. cendré. Taillis humides du pied. Mars, Avril.

"S. grundifoliu Ser. S. à grandes feuilles. Taillis audessus de Grevin, d'Archamp, etc. Avril, Juin.

S. cupraea L. S. Marceau. Taillis du pied. Mars, Avril.

S. auritu L. S. auriculé. Taillis du pied. Mars, Avril.

S. repens L. S. rampant. Marais de Veyrier, de Bossey. Avril, Juin.
Populus.
Peuplier.

P. alba L. P. blane. Au bas du chemin de la Groiselte. Mars, Avril.

P. Iremula L. P. Tremble. Taillis du pied. Mars, Avril.

\section{Hetulinece.}

Betula.

\section{Bétulinées.}

Bouleau.

"B. alba L. B. blanc. Revers méridional au-dessus de Cruseilles. Avril, Mai.

\section{Alnus.}

Aune.

*A. viridis DC. (Betula viridis Chaix). $\Lambda$. vert. Derrière les Pitons et au-dessus de Cruseilles. Mai, Juin.

A. incana DC. (B. Alnus $\beta$ incana L.) A. blanchâtre. Bords de l'Arve, ruisseaux du pied. Février, Mars. 
A. glutinosa Gaert. (B. Alnus $\gamma$ glutinosa L.) A. glutineux, Verne. Mèmes localités. Février, Mars.

\section{Coniferae.}

Taxus.

\section{Conifè res.}

If.

"T. baccata L., If commun. Cỉ et là, sur les pentes du versant occidental. Mars, Avril.

Juniperus. Genévrier.

$J$ communis L., G. commun. Rocailles du pied et de la région moyenne. Avril, Nai.

Pinus.

Pin.

P. sylvestris L., P. sauvage. Bois du pied. Mai, Juin.

* $P$. uncinata Ram., God. (P. sylvestris L. o pumilio Gand.) découvert au-dessus du Crevin par MM. Reuter et Rapin, en 1866, rare. Mai, Juin.

Abies.

Sapin.

*A. excelsa. DG. (Pinus Abies L., Gaud.) S. élevé, S commun, S. noir, Epicea, Pesse. Bois de la montagne. Mai, Juin.

A. pectinata DG. (P. Picea L. Gaud.) S. blane, S. argenté. Bois de la montagne, plus rare que le précédent. Mai, Juin.

Obs. Le premier a les fenilles tétragones, éparses et les cônes pendants : le second a les feuilles planes, distiques, émarginées et les cônes drossés. 


\section{MONOCOTYLEDONES.}

\section{Alismacerue.}

Alisma.
Alismacées.

Flûteau.

A. Plunlugo L., F. Plantain-d'eau. Narais près des chalets. Juillet, Septembre.

Enecayinere.

Triglochin.

\section{耳uncaginées}

Troscart.

E. palustre L., T. des marais. Endroits marécageux du pied, au-dessus d'Archamp. Juin, Août.

Potnmene.

Potamogeton.
Potanáes.

Potamol.

P. plantagineus Ducr. (G. Ilornemanni Mey.) G. à feuilles de plantain. Marais de Veyrier. Mai, Juin.

'P. densum L. $\beta$ lanceolatum Lioch. P. serré. Mares du sommet, vers les Pitons. Juillet, Octobre.

\section{Anoidene.}

Arum.

\section{Aroidées.}

Gouel.

A. maculatum L., G. tacheté, Pied-de-Veau. Taillis du pied. Avril, Mai.

\section{Typhereece.}

Typlia.

\section{Typhacées.}

Massette.

T. minimu Hopp., II. naine. Sable d'Arve au-dessous de Veyrier. Avril, Mai. Refleuril quelquefois en automne. 


\section{Sparganium. Rubanier.}

S. simplex Iluds. (S. erectum $\beta$ L.) R. simple. Marais de Viry (II. Chavin) Juin, Juillet.

S. minimum Fries. (S. natans Gaud. non L.) R. nain. Marais de Viry (II. Chavin). Juin, Juillet.

orebidere.

Orchis.
Drehidées.

Orchis.

O. Morio L., O. Bouffon. Monticules découverts du pied. Avril, Mai.

O. coriophora L. 0. punais. Marais de Troinex et de Veyrier. Mai, Juin.

O. ustuluta L. O. brúlé. Monticules découverts du pied. Avril, Mai.

O. Simia Lam. (0. militaris $\beta$ Gaud., 0. Tephrosanthos

Vill.) 0. singe. Taillis du pied. Mai, Juin.

O. galeuta Lam. (0. militaris $\propto$ L., Gaud.) 0 . en casque. Clairières des bois de Veyrier, de Crevin, etc. Mai, Juin.

O. purpurea Huds. (0. fusea Jacq., 0. militaris DC.) 0. pourpré. Taillis du pied. Mai, Juin.

O. muscula L., O. mâle. Buissons du pied et pìturages de la région moyenne. Avril. Juin.

* $O$ globosa L., O. globuleux. Paturages du sommet. Juin, Juillet.

"O. sambucina L., 0. Sureau. Pâturages vers les Pitons, rare. (M. P. Privat). Juin, Juillet.

O. lalifolia L., Koch. (0. maialis Reich) 0 . à larges feuilles. Prairies humides du pied et de la région moyenne. Nai, Juin.

O. incarnala L. (0. angustifolia W. et G., Koch.) 0 . incarnat. Marais de Veyrier, Bossey, etc. Mai, Juin.

- O. Traunsteineri Saut. Koch. (0. augustifolia Fries, 0. incarnata $\beta$ angustifolia Reich., Gr. el G., God.) 0. 
de Traunsteiner. Petits marais an-dessus d'Archamp. Nai, Juillet.

O. maculata L., 0 tacheté. Bois du pied et de la région moyenne. Mai. Juillet.

Anacamplis.

Anacamptis.

A. pyrrmidalis Rich., Koch. (Orchis pyramidalis $\mathrm{L}_{\text {. }}$ )

A. pyramidal. Bois de Crevin. Juin, Juillet.

Gymnadenia. Gymnadénie.

G. conopsea Rich. (0. conopsea L.) G. à long éperon. Bois el prairies du pied et de la région moyemne. Juin, Juillet.

"G. odoratissima Rich. (0. odoratissima L.) G. odorante. Au bas de la Grande-Gorge, au-dessus d'Archamp. Juin, Juillet.

"Gr. albida Rich. (Satyrium albidum L., Orchis albida All.) G. blanchàtre. Piturages du sommet, vers les Pitons. Juin, Août.

Platanthera.

Platanthère.

P. lifolia Rich. (Orchis bifolia L. Gaud.) Taillis du pied. Juin, Juillet.

* P. chlorantha Gust. Reich. Koch. ( 0 . bifolia $\beta$ elatior Gaud. 0. virescens Zoll. 0. montana Schm. Gr. et G.) P. verdittre. Taillis of hois dı pied. Mai, Juillet.

* $P$. viridis Lindl. (Satyrium viride L. Orchis viridis All. IIabenaria viridis R. Br. Gymnadenia viridis Rich.) P. verte. Pâlurages de la montagne. Mai, Juillet.

Ophrys.

Ophrys.

O. muscifera Iluds. (0. myodes Jaeq. Gaud. 0. insectifera et myorles L.) 0. Nonche. Monticules du pied. Mai, Juin.

O. armiferu Hurls. 0. Araignéc. Monticules du pied. Avril, Mai. 
O. arachnites Reich. (0. fucifera Reich. God.) 0. Bourdon. Clairières du pied. Mai, Juillet.

0 . apiferc Huds. 0 . Abeille. Clairières et monticules du pied. Mai, Juin.

\section{Herminium. Herminie.}

H. Monorchis R. B. (Ophrys Monorchis L.) II. à un seul tubercule. Près de Veyrier. Juin, Juillet.

\section{Nigritella.}

Nigritelle.

* $N$. angustifolia Rich. (Satyrium nigrum L., Orchis nigra All.) N. à feuilles étroites, Vanille de montagne. Pâturages du sommet. Juin, Juillet.

* $N$. nigro-conopsea Reich. Forme hybride rare et trouvée par M. Rapin au-dessus de la Croisette. Juillet.

\section{Limodorum. Limodore.}

L. abortivum Sw. (Orchis abortiva L.) L. à feuilles avortées. Bois de Grevin, rare. Juin, Juillet.

Epipactis. Epipactis.

E. latifolia All. Koch. (E. latifolia a pallens Gaud. Serapias latifolia L.) E. à larges feuilles. Buissons du pied. Juillet, Août.

${ }^{*}$ E. rubiginosa Koch. (E. latifolia $\beta$ rubiginosa Gaud. E. atrorubens Hoffm.) E. rougeâtre. Buissons du pied. Juillet, Aoûl.

E. palustris Cr. (Serapias longifolia L.) E. des marais. Marais de Troinex, de Collonge, d'Archamp. Juin, Juillet.

\section{Cephalanthera. - Céphalantère.}

C. pallens Rich. Koch. (Epipactis grandiflora Gaud. E. lancifolia DG. E. pallens Sw.) G. à grandes fleurs. Bois du pied, sous les pins. Mai, Juin.

C. ensifolia Rich. (Epipactis ensifolia Sw.) C. à fenilles ensiformes. Bois de Grevin. Mai, Juin 
C. mbra Rich. (Epipactis rubra All.) C. rouge. Bois du pied. Juin, Juillet.

\section{Neottia.}

Néottie.

*N. Nidus-avis Rich. Gaud. (Ophurys Nidus-avis L.) Bois de sapins de la montagne. Mai, Juin.

\section{Lislera.}

Listère

L. ovatu R. Br. (Ophrys ovata L. Epipactis ovata All.) L. it feuilles ovales. Bois du pied. Juin, Juillet.

Spiranthes.

Spiranthe.

S. restivalis Rich. (Neottia aestivalis DC.) S. d'été. Marais au-dessus de Collonges, marais de Bossey, de Veyrier. Juillet, Août.

S. autumnulis lich. (Ophrys spiralis L. Neottia spiralis Sw.) S. d'automne. Veyrier. Septembre, Octobre.

\section{Goodyera.}

Goodyère.

G. repens R. Br. (Satyrimm repens L. Neottia repens IJ.) G. rampante. Bois de pins, au-dessus de Grevin et d'Archamp, rare. Juillet, Août.

\section{Liparis. Liparis.}

L. Loeselii Rich. (Ophrys Loeselii L. Malaxis Loeselii Sw.) L. de Loesel. Marais de bossey (M. Iluet), rare. Juin, Juillet.

Corallorhiza.

Coralline.

* C. innulu R. Br. (C. IIalleri Rich. Ophrys corallorhiza L.) (i. de Ilaller. Forêt de sapins près du Grand-Piton (M. Ramu), rare. Juin, Juillet.

Cypripedium.

Sabol.

* C. culccolus L. S. de Vénus. Taillis du pied entre Collonges at Pommier, chemin de la Croisette. Mit, Juin. 


\section{rovialere.}

Crocus.

\section{Inidées.}

Safran.

* G. vernus All. S. printanier. Pâturages du sommet, entre la Croisette et les Pitons. Avril. Mai.

\section{Amuryllidere.}

Narcissus.

\section{Anaryllidées.}

Narcisse.

N. Pseudo-Narcissus L. N. faux-Narcisse. Ver's lit Pierre-aux-Fées. Mars, Avril.

$N$. poeticus L. N. des Poètes. Çà et là dans les prairies, au-dessus de Crevin et de Collonges. Mai, Juin.

N. biflorus Curt. N. à deux fleurs. Dans les prés et au bord des champs à Troinex, Evordes, Collonges. Avril, Mai.

\section{Lenooium. Niveole.}

${ }^{*} L$. vernum L. N. du printemps. Perce-Neige. Pentes du Petit-Salève à gauche du Pas-de-l'Echelle, Château d'Etrembières, Voûtes supérieures, Vallée des Usses. Février, Mars.

\section{Asprescugene.}

Paris.

\section{Asparagées.}

Parisette.

P. quadrifolia L. P. à quatre fenilles. Taillis du pied. Mai, Juin.

Convallaria.

Muguet.

${ }^{*} C$. verticillata L. II. verticillé. Taillis au-dessus d'Archamp, de Grevin. Mai, Juin.

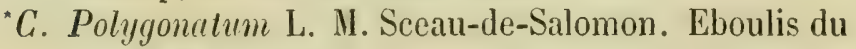
pied. Mai, Juin.

*C. Maialis L. M. de Mai, Lis des vallées. Taillis du pied. Avril, Mai.

Maianthemum. Maianthème.

"M. bifolium DG. (Convallaria bifolia L.) M. à deux feuilles. Taillis et rocailles du pied. Mai, Juin. 
Ruscus.

Fragon.

"R aculealus L. F. piquant, Petil-IIoux. Trouvé une fois au Pelit-Salève, à chercher. Mars, Avril.

\section{miosconece.}

Tamus.
Dioscorées.

Taminier.

T. communis L. T. commun, Coulourrée noire. Taillis du pied. Mai, Juin.

\section{titincene.}

Tulipa.

\section{Liliacées.}

Tulipe.

T. sylvestris L. T. sanvage. Au pied d'une hate entre Sierne et Veyrier, champs ì Neydans. Avril, Mai. Lilium. Lis.

* L. Martugon L. L. Martagon. Taillis du pied et de la région moyenne. Mai, Juillet.

Erythronium.

Erythrone.

E. Dens-canis L. E. Dent-de-Chien. Bois entre la Pierre-aux-Fées et l'Arve. Mars, Avril.

Ornithogalum.

Ornithogale.

O. myrenaicum L. 0. des Pyrénées. Taillis rocailleux du pied. Mai, Juin.
Gagea.
Gagée.

G. arvensis Schult. Koch. (G. villosa Dub. Ornithogalum arvense Pers. O. minimum DG.), G. des champs.

Veyrier dans les champs. Mars, Avril.

G. luleu Schult. Koch. (G. lutea $\beta$ sylvatica Dub. Ornithogalum luteun $\beta$ L. O. sylvalicum Pers.) G. jaune. Prés d'Ttrembières, au bord de la route de Mornex, près de Veyrier, à Crevin. Avril, Mai.

Scilla. $\quad$ Scille.

S. bifolia L. S. à deux feuilles. Haies et buissons du pied. Mars, Avril.
Muscari.
Muscari.

M. racemosum, Mill. (Ilyacinthus racemosus L.) M. à grappes. Vignes à Mornex. Avril, Mai. 
M. comosum Mill. (II. comosus L.) M. chevelu. I'ied de la montagne. Mai, Juin.
Allium.
Ail.

A. ursimm L. A. des Ours. Endroits humides du pied. Avril, Mai.

A. sphaerocephalum L. $\Lambda$. à tète ronde. Rocailles audessus du Pas-de-l'Echelle, au Coin. Juin, Juillet.

A. carinatum L. A. caréné. A Veyrier, près de l'Arve. Juillet, Août.

\section{Phalangium. Phalangère.}

P. Liliago Schr. (Anthericum Liliago L.) P. fiux-Lis. Grand et Petit-Saleve, au-dessus-de Monetier et de Iíornex, au-dessus du Coin. Juin, Juillet.

P. rumosum Lam. (A. ramosum L. Gaud.) P' rameuse. Taillis du pied. Juillet, Août.

\section{Coteleiercere.}

Colchicum.

\section{Colchicacées.}

Colchique.

C. autumnale L. C. d'automne, Tue-chien. Prairies du pied et de la région moyenne. Septembre, Octobre. Tofieldia.

Tofieldie.

T. palustris Huds. (T. calyculata Wahl. Koch. Anthericum calyculalum L.) Endroits marécageux du pied et de la région moyenne. Juin, Juillet.

\section{-trencrecere.}

Juncus.

\section{Honcacées.}

Jone.

J. glaucus Ehrh. (J. intlexus Leers. DC.) J. glauque.

Lieux humides, depuis Ic pied jusque vers les sommets. Juin, Juillet.

J. obtusiflorus Ehr. J. a fleurs obtuses. Marais du pied, Troinex, Bossey. Juillet, Août.

J. alpimus Vill. J. des Alpes. Bords de l'Arve sous Veyrier. Juin, Septembre. 
Luzula.

Luzule.

"L. Alevescens Gaud. L. jaunitre. Bois de sapins de la montagne. Nai, Juin.

"L. maxima DC. (L. sylvatica Gaud.) L. géante. GrandeGorge et vers les Pitons. Juin, Juillet.

L. nivea DG. L. blanche Bois du pied, au-dessus d'Archamp. Mai, Juin.

\section{Cypenerene.}

Schœnus.

\section{Cypéracées.}

Choin.

S. nigricans L. C. noiràlre. Marais de Troinex, Bossey. Mai, Juin.

Cladium.

Cladie.

G. Mariscus R. B. (Schœnus Mariscus L.) C. Marisque. Marais de Troinex. Juin, Juillet.

Heleocharis. Héléochare.

H. acicularis R. Br. (Scirpus acicularis L.) H. aciculaire. Clairières humides du bois de Veyrier. Juin, Août.

Scirpus.

Scirpe.

S. pauciflorus Ligth. Koch. (S. Baeothryon Ehrh. Gaud, S. Halleri Vill.) S. pauciflore. Petits marais audessus de Collonge, d'Archamp. Juin, Août.

S. setacens L. (Isolepis setacea R. Br.) S. sétacé. Bois de Viry (II. Chavin). Aoùt, Septembre.

"S. compressus Pers. (Schœnus compressus L.) S. comprimé. Au-dessus de Collonges et fontaine des Pitons. Mai, Juin.

Eriophorum.

Linaigrette.

E. angustifolium Roth. Gaud. L. à feuilles étroites. Marais du pied el de la région moyenne. Avril, Mai.

${ }^{\star} E$. vaginatum L. L. à larges gaines. Petit marais tourbeux derrière le Grand-Piton. Mai, Juin. 
Carex.

Laiche.

'C. dioica L. L. dioirque. Marais du pied, Bossey, Collonges. Avril, Mai.

C. Daralliane Sm. L. de Davall. Petits marais du pied. Avril, Mai.

C. paniculata L. L. paniculée. Bords de l'Arve près d'Etrembières. Avril, Mai.

C. leporina L. (G. ovalis Good.) L. des Lièvres. Bois du pied. Nai, Juin.

*C. stellulata Good. L. étoilée. Marais derrière le GrandPiton. Mai, Juin.

C. remota L. L. espacée. Endroits humides au-dessus de Bossey, d'Archamp. Mai, Juin.

*C. vulgaris Fries. (C. cosspilosa Gaud. non L. C. Goodenowii J. Gay, d'après Renter.) L. comm mune. Narais derrière le Grand-Piton. Juin, Juillet.

C. montana L. L. des montagnes. Taillis du pied. Mars, Avril.

C. praceox Jacq. L. précoce. Rocailles du pied. Mars, Avril.

- C. gynobasis Vill. (G Ialleriana $\Lambda$ ss. Gr. et G. G. alpestris All.) L. gynobase. Rocailles du pied, autdessus de Grevin, du Coin. Avril, Mai.

C. humilis Leys. (C. clandestina Good. Gaud.) L. naine.

Sommel de la Petite-Gorge, rare. Avril, Mai.

C. digitatu L. L. digitée. Bois du pied. Mars, Avril.

"C. ormithopoda Willd. (C. pedata DC. fl. fr.) L. Picdd'Oiseau. Taillis rocailleux du pied. Avril.

*C. alba Scop. I. blanche. Taillis du pied. Avril, Mai. C. nitida Huds. L. lustrée. Sables au bord de l'Arve près d'Etrembiẻres. Avril, Mai.

C. glanca Scop. L. glauque. Endroits humides du pied, s'élève assez haut. Avril, Mai. 
C. maxima Scop. (C. pendula Huds.) L. géante, près de Crevin et d'Archamp. Mai, Juin.

C. pallescens L. L. pâle. Bois du pied et de la région moyenne. Mai, Juin.

"C. sempervirens Vill. (C. erecta DC.) L. verte. GrandeGorge, Pitons. Mai, Juin.

"C. tenuis Ilost. (C. brachystachys Schr.) L. grêle. Rochers du Grand-Piton, rare. Mai, Juin.

C. flava L. L. jaune. Marais du pied. Avril, Mai.

C. lepidocarpa Tausch. L. écailleuse. Marais au-dessus de Bossey, Collonges. Mai, Juin.

G. Hornschuchiana Hopp. (C. fulva DC. non Good. d'après Reuter.) L. d'Ilornschuch. Marais au-dessus de Collonges. Avril, Mai.

$\beta$ xanthocurpa God. (G. fulva Good. Bor. d'après

Reuter.) Prairie marécageuse au-dessus de Collonges. Avril, Mai.

C. sylvatica Huds. Gaud. (C. patula Scop. DG.) L. des bois. Taillis du pied. Mai, Juin.

C. ampullacea Good. L. ampoulée. Bords de l'Arve près d’Etrembières. Avril, Mai.

\section{cormineare.}

Phalaris.

\section{Graminces.}

Nlpiste.

P. arundinacea L. Gaud. A. Roseau. Marais de Troinex. Juin, Juillet.

\section{Anthoxanthum.}

Flouve.

A. odoratum L. F. odorante. Bois et prairies du pied et de la région moyenne. Avril, Mai.

$\beta$ villosum Reich. Cuà et là avec le type. Avril. Mlai.

\section{Phleum.}

Phléole.

P. pratense L. $\gamma$ nodosum Gaud. (P. nodosum L.) P. des prés, var. tuberculeuse. Champs des TreizeArbres, de la Croiselte. Juin, Août. 
* $P$. alpinum L. P. des Alpes. Pàturages des sommets. Juillet, Août.

P. Boclemeri Wib. (Phalaris phleoides L., Phleum phalaroides Koel. Gaud.) P. de Boehmer. Monticules du pied, au-dessus de Veyrier. Juin, Juillet.

\section{Sesleria. Seslérie.}

S. coerulea Ard. (Cynosurus coeruleus L.) S. hlenatl'e.

Pâturages secs du pied et de la région moyenne. Avril. Juin.

Setaria.

Sétaire,

S. glanca P. B. (Panicum glaucum L.) S. glaurne.

Cultures sous Veyrier, près de l'Arve. Aoûl, Septembre.

Panicum.

Panic.

P. glabrum Gaud. (Digitaria filiformis Kocl.) P. glitbre. Cultures sous Veyrier, près de l'Arve. Août. Septembre.

Andropogon. Barbon.

A. Ischuemum L. B. Pied-de-P'oule. Lindroits arides du pied. Août, Septembre.

Calamagrostis. Calamagrostide.

G. Epigeios Roth. (Arundo Epigeios L.) C. commune sous les saules au bord de l'Arve, près de Veyrier et d'Etrembières. Juillet, Août.

C. littorea DC. C. des rivages. Mélangée avec la précédente. Juillet, Août.

"C. montuna Host., C. des montagnes. Bois du pied el de la région moyenne. Juillet, Août.

$$
\text { Agrostis. Agrostide. }
$$

A. alba L., y gigantea Reut. (A. gigantea Gaud.) A. blanche, var'. géante. Au-dessus d'Archamp. Juillet, Septembre. 
A. vulgaris With., A. commune. Prairies du pied et de la région moyenne. Juillet, Août.

$\gamma$ pumila Gaud., Reut. Allées du bois de Veyrier. Juillet, Août.

A. intermpte L. (Apera interrupta I', B.) A. interrompue. Veyrier, vers la croix et autour de la gravière. Juin, Juillet.

$$
\text { Stipa. Stipe. }
$$

"S. pennata L. , S. plumeuse. Rochers du Grand-Salève près de la Grande-Gorge, Petit-Salève au-dessus d'Etrembières. Mai, Juin.

Lasiagrostis. Lasiagrostide.

"L. culamagrostis Link. (Galamagrostis argentea DC. Stipa calamagrostis Gaud.) L. argentéc. Rocailles du Pas-de-l'Echelle. Juin, Juillet.

Milium.

Millet.

"M. cffusum L., M. étalé. Bois de la montagne. Juin, Juillet.

Deschampsia. Deschampsie.

*ID. Alexuosu Griseb. (Lira llexuosa L. Avena flexuosa M. el K.) D. flexueuse. Pìturages sablonnenx du sommet, ver's les Pitons et au-dessus de Pommier. Juillet, Août.

Avena. Avoine.

A. fulua L., A. folle, Folle-avoine. Champs à Monetier. Juin, duillet.

A. mutensis L., $\Lambda$. des prés. Avenette. Chemin de la Croiselte. Juin, Juillet.

Arrhenatherum.

Arrhénatère.

A. clatius N. et K. (Avena elatior L., Gaud.) A. élevée, Fromental. Prairies du pied el de la région moyenne. Mai, Juin. 
Triselum.

Triséte.

T. flacescens P. B. (Avena llavescens L.) T. jaunitre. Pâturages du pied et de la région moyenne. Juillet, Aoùt.

Koeleria.

Koelérie.

K. cristata Per's. (Aira cristata L.) K. à crètes. P'ilu.. rages du pied el de la région moyenne. Juin, Juillet.

Glyceria.

Glycérie.

G. plicute Fries, G. pliće. Mares du sommet, Collonges, le Châble. Juin, Juillet.

\section{Poa.}

Pàturin.

P. annua L., P. annuel. Prairies, surtout autour des Chalets. Nlai, Septembre.

*P. alpina L., P. des Alpes. Pâturages du sommet. Mai, Juillet.

" $\beta$ brevifolia Gr. et G. Sur les Pitons. Mai, Juillet.

$P$. nemoralis L., G. des bois. Taillis du pied et de la région moyenne. Juin, Juillet.

" $\beta$ rigidulu Gaud. (P. coaretata DG.) Bois de la montagne. Juin, Août.

\section{Briza.}

Brize.

B. mediu L., B. moyenne, B. tremblante, Anourelte. Pàturages secs du pied el de la région moyenne. Juin, Juillet.

\section{Melica.}

Mélique.

M. ciliata L. Gaud. (II. Nebrodensis Gr. et G.)M. ciliée.

Eboulis du pied. Juin, Juillet.

M. nutans L., M. penchée. Taillis rocailleux du pied. Avril, Mai.

M. uniflora Retz. N. uniflore. Rocailles du pied. Mai, Juin. 
Dactylis.

Dactyle.

D. glomerulu L. D. pelotonné. Prairies sèches du pied. Mai, Août.

Scleropoa.

Scléropoa.

S. rigidu. Griseb., Gr. et G. (Poa rigida L., Fesluca rigida Kunth., Koch.) S. roide. Rocailles du pied. Août, Septembre.

\section{Molinia. Molinie.}

M. litloralis Host., Reich. (II. cocrulea Moench $\beta$ altissima God.) M. des rivages. Endroits humiles du pied. Aoùt, Septembre.

\section{Triodia. \\ Triodie.}

T. decumbens P. B. (Festuca decumbens L., Danthonia decumbens DC.) T. inclinće. Marais de Veyrier, de Troinex. Juin, Juillet.

Cynosurus. Cretelle.

C. cristutus L., C. crêtée. Pàturages du pied et de lat région moyenne, Juin, Juillet.

$$
\text { Vulpia. Vulpie. }
$$

V. Pscudo-myuros Gay. Gr. et G. (Festuca Pseudo-Myuros S. Will., F. Myuros Poll. Gaud.), V. Quene-deRat. Gravière de Veyrier. Mai, Juin.

V. ciliata Link. (V. Myuros Reich., Festuca ciliata Pers.)

V. ciliée. Eboulis du Petit-Salève au-dessus de Veyrier, sables d'Arve près d'Etrembières (M. Rapin). Mai, Juin.

\section{Festucu.}

Fétuque.

$F$. ovina L. F. des brebis. Prairies du pied el de la région moyenne. Mai, Juin.

F. tenuifolia Sibt. (F. ovina L. y tenuifolia Dub.) F. a feuilles menues. Au pied des grandes roches d'Archamp, à droite de la Serratula (II. Bernett). Nai, Juin. 
"F. glanca Lam. (F. duriuscula L. $\beta$ glauca Rap.) F. glauque. Sur les Pitons. Juin, Juillet.

F. heterophylla Lam. F. hétérophylle. Bois de Veyrier. Juin, Juillet.

* F. sylvatica Vill. F. des forèts. Bois au-dessus d'Archamp, de Pommier. Juillet, Août.

$F$. prutensis IIuds. (F. clatior L) F. des prés. Prairies, surtout autour des chalets. Juin, Juillet.

F. gigantea Vill. (Bromus giganteus L.) F. géante. Bois humides du pied. Iuillet, Septembre.
Bromus.
Brome.

B. sterilis L. B. stérile. Yoùtes du Petit-Salève. Avril, Mai.

B. erectus Huds. B. dressé. Prés sees du pied et de la région supérieure. Mai, Juin.

B. asper L. B. rude. Taillis du pied. Juin, Juillet.

\section{Elymus.}

Elyme.

${ }^{\star} E$. Europaeus L. E. d'Europe. Bois au-dessus d'Ar'champ, de Pommier. Juillet, Août.

\section{Trilicum. Froment.}

T. coninum Schr. (Agropyrum caninum R. el S.) F. des chiens, F. des haies. Taillis du Pas-de-l'Echelle, chemin de la Croisetle. Juin, Juillet.

\section{Lolium. Ivraie.}

L. strictum Presl. Gr. et G. (L. rigidum Gaud.) I. roide. Champs de Moneticr. Mai, Juin.

'L. temulentum L. I. enivrante. Champs de la montagne. Juin, Août.

Nardurus.

Nardure.

N. tencllus Reich. Gr. et G. (Triticum Nardus DC. Gaud. Festuca ternuiflora Schr. Koch.) N. délicate. Pas-de-l'Echelle, carrières de Mornex, rare. Mai, Juin. 
Nardus.

Nard.

"N. stricta L. N. raide. Pâturages des sommets. Mai, Juillet.

Obs. On cultive dans tous les villages: Zea, Mays L., Naïs, Blé de Turquie. Avena sativa L. A. cullivée. A. orientalis Schr. A. d'Orient, A. de IIongric. Hordeum. vulgare $\mathrm{L}$. Orge commun, 0 . à quatre rangs, $H$. hexastichum L. 0 . à six rangs, 0 . carré, 0. distichum L. 0 . à deux rangs, 0 . plat, II. Zeocriton L., 0. Riz, 0. pyramidal, Secale cereale L., Seigle cultivé, Triticum vulgare Vill. Froment cultivé, blé, T. Spelta L. F. Epeautre. T. monococcum L. F. locular, petite Epeautre. Dans les villages, le long des murs, près des fumiers, dans les cultures et autour des chalets, croissent: Seturiu viridis P. B. Sétaire verte, Punicum sanynimale L. Panic purpurin, P. Crus-Gulli L. P. Pied-de-Coq. et Hordeum murinum L. 0. des murs, Queue de souris.

Dans les prairies, se trouvent la plupart des Graminées de la plaine, entr'autres: Agrostis vulguris With. Agrostide commune, Por trivialis L. Patr:rin commun, P. prutensis L. P. des prés, Lolium perenne $\mathrm{L}$. Ivraic vivace, etc. 


\section{ACOTYLÉDONES.}

\section{Filices.}

Botrychium.

\section{Fiagèces.}

Botrychée.

*B. Lunuria Sw. (Osmunda Lunaria L.) B. Lunaire, Pâturages du sommet. Juin, Juillel.

\section{Ceterach.}

Ceterach.

*C. officinarum C. B. C. commun. Rochers à Mornex, vis-à-vis du Mont Gosse (Reuler). Murs en pierres sèches, entre la Mûre et Mornex (Rapin). Septembre, Octobre.

Polypodium.

Polypode.

P. vulgare L. P. commun, Réğlisse de monlagne. liochers ombragés du pied. Septembre, Novembre.

Phegopteris.

Phégoptère.

"P. Dryopteris Fée. Bernouil. (Polypodium Dryopteris L.) P. dryoptère. Bois de sapins, atl-dessus d'Archamp. Juillet, Octobre.

P. calcarea Fee. Bern. (Polypodium calcareum Sm.

P. Robertianum Iloffm. Koch.) P. calcaire. Rochers ombragés du pied. Juillet, Octobre.

\section{Aspidium.}

Aspidie.

"A. Lonchitis Sw. (Polypodium Lonchitis L.) A. lonchite. Rocailles de la montagne, Pitons. Juillet, Octobre. 
"A. aculeatum Doell. Koch. (Polypodium aculeatum L. Polystichum aculeatum Roth.) A. aiguë. Bois de sapins. Juillet, Octobre.
Polystichum.
Polystic.

P. Thelipteris Roth. (Polypodium Thelipteris L. Aspidium Thelipteris Sw. Bern.) P. Théliptère. Marais de Troinex. Août, Octobre.

${ }^{*} P$. spinulosum DC. (Aspidium spinulosum Doel. A. dilatatum Godr.) P. spinuleux, avec les var. $\alpha$ vulgare $\mathrm{Gr}$. et $\mathrm{G}$. et $\beta$ dilatatum $\mathrm{Gr}$. et $\mathrm{G}$. Bois de sapins de la montagne. Juillet, Octobre.

*P. Filix-Mas DG. (Aspidium Filix-Mas Sw. Bern., Polypodium Filix-Ilas L.) P. Fougère mâle. Bois de sapins. Août, Octobre.

*P. rigidum DC. (Aspidium rigidum Sw. Polypodium fragans Vill.) 0. raide. Sommet du Grand-Piton, rare. Juillet, Octobre.

Cystopteris.

Cystoptère.

C. fregilis Bernh. (Aspidium fragile Sw. Polypodium fragile L.) C. fragile. Rochs's ombragés du pied. Juillet, Octobre.

Asplenium.

Doradille.

A. Filix-femina Bernh. Koch. (Polypodium Filixfomina L. Alhyrium Filix-fuemina Rolh.) D). Fougère femelle. Bois de la montagne. Août, Octobre.

"A. Halleri DG. D. de Haller. Rochers ombragés du pied. Juillet, Octobre.

A. Trichomanes L. D. Polythric, Capillaire rouge. Rochers moussus du pied, vieux murs. Juillet, Octobre.

A. viride Iluds. D. verte. Rocailles du pied. Juillet, Octobre.

A. Ruta-muraria L. D. des murs. Vienx murs et tochers du pied. Juillet, Octobre. 
A. Adrunthum-nigrum L. D. noire, Capillaire noire.

Rocailles du chemin des Chitaignier's, rochers audessus du chàteau d'Etrembières. Août, Octohre. "A. septentrionale Sw. (Acrostichum septentrionale L.). D. septentrionale. Blocs erraliques, Esery, Croiselle, versant oriental, au-dessus de la llûre (II. Rapin). Août, Octobre.

Scolopendrium. Scolopendre.

"S. officinamm Sw. (Aspleniun Scolopendrium L). S. officinal, Langue-de-Cerf. Rochers oubragés dı pied, Pas-de-l'Echelle. Aoùt, Octobre.

\section{Blechnum. Blechne.}

'B. spicant Roth. (Osmunda spicant L.) B. commun. Bois de sapins du côté méridional. Août, Octóbre. Pleris. Ptéride.

P. aquilina L. P. aigle, Fougère impériale. Bois du pied, s'élève jusqu'au sommet. Septembre, Octobre.

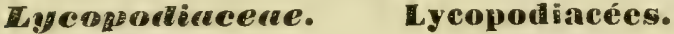

Lycopodium.

Lycopode.

" $L$. sclago L. sélagine. Sommet près de la Croiselle et de la Grande-Gorge. Juillet, Août.

' $L$. clncutum L. L. en massue. Conches sidérolitiques vers Cruseilles et près de la Grande-Gorge (II. Bernell). Juillet, Août.

Selaginella.

Sélaginelle.

'S. sprimulosa R. Br. (Lycopodium sclaginoides L.) S. épineuse. Patturages près de la Grande-Gorge et des Pitons. Juin, Juillet.

\section{Equesetrcere.}

Equisetum.

\section{Equisétacées.}

Prêle.

E. Telmatein Ehrh. (E. fluviatile Sm.) P. des rivières. Endroits marécageux du pied. Avril, Mai. 


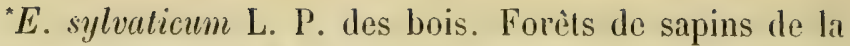
montagne. Mai, Juillet.

E. pulustre L. P. des marais, Quene de cheval. Prairies humides du pied el de la région moyenne. Juin, Août.

E. limosum L. P. des bourbiers. Marais de Troinex. Juin, Juillet.

E. vuriegatum Schl. (E. multiforine $\alpha$ Vauch.) P. panachée. Sables d'Arve, près de Veyrier. Juin, Août.

E. hyemale L. P. d'hiver. Endroits humides dı pied. Juillet, Août.

$\beta$ paleacenm Doel. Bern. Près du Châble. Juillet, Août.

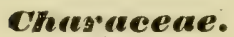

Nilella.

\section{Characées.}

Nitelle.

N. copitate $\Lambda \mathrm{g}$. (Chara capitata Nees. C. syncarpa Thuil) N. capitée. Mare au-dessus du bois de Crevin. Avril, Juin.

N. opuca $\mathrm{Ag}$. A. Br. N. opaque. Même localité. Juin, Août.

N. polysperma Kütz. N. polysperme. Mares du pird de la monlagne. Juin, Août.

N. glomerata Ag. A. Br. N. agglomérée. Mare an-dessus de Grevin. Juin, Août.

\section{Chara.}

Charagne.

C. hispide L. G. hispide. Marais de Veyrier, mare de Grevin. Juin, Août. 
NOTES 



\section{NOTES}

sur les plantes les plats intéressantes du Salève

\section{Atragene alpina L.}

\section{Syn. : Clematis alpina Mill. DC.}

Celle stiperbe espèce se trouve en abondance dins denx localités rapprochées, parmi les éboulements calcaires au-dessus du village d'Archamp. Ses tiges sarmenteuses et les guirlandes azurées de ses grandes et belles fleurs recouvrent les différents arlustes qui croissent au nilien du cahos des blocs détachés du flanc de la montagne. On m'a dit qu'il en existe quelques pieds dans l'entonnoir de la Petite-Gorge et j'engage les jeunes botanistes à vérifier cette assertion. La station la plus voisine du Salève est aux escarpements d'Andey près de Bonneville, d'après II. Dumont.

Ilaller signale plusieurs localités des Grisons, d'après Bauhin, Gesner, Scheuchzer et Dick.

Suler indique le Munsterthal, l'Albula, le mont T'schiera, le Splugen et le mont Rufeln.

Gaudin ajoute que depuis Ifaller la plante a ćté trouvée abondamment à Salève par les hotanistes genevois. Ilegetschweiler (Flora der Schweiz 1840) l'indique sur presque toutes les Alpes des Grisons depuis 4 à 6000 pieds d'élévation (Saint-Mlorilz dans la IIantc-Engadine; Saint-Antoine dans le Prettigau; l'Oberalp, ete.), cnfin le Mont-Salève. Moritzi (Flora der Schweiz 1844) donne plusieur's localités des Grisons ainsi que le Salève. Godet, Rapin, Reuter, signalent notre montagne, et ce dernier ajoute les escarpements d'Andey près de Bonneville. 
D'après Allioni (Flora Pedemontanit) elle est ahondante dans les Mlpes de Fénestrelle et au Mont-Cenis à l'endroit appelé Los Laros.

Reichenbach (Flora Germanica exeursoria) signale les Alpes et les vallées alpestres de l'Autriche et de l'Allemagne méridionale. Koch (Synopsis Floræ Germanicae et Helvelicae) indique la Suisse et toute la chaine dos Mlpes jusqu'en Autriche. Villars (Histoire des plantes du Dauphiné), De Candolle, (Flore Françaiso) Lapeyrouse (IIist. abrégée des plantes des Pyrénées), Ilutel (Flore du Dauphiné). Loiscleur des Longchamps, Mutel (Flore de France), Grenier et Godron (Flore de France). Philippe (Flore des Pyrénées), signalent les Alpes du l'iémont, celles du Dauphiné, de la Provence, le Canigon et les Pyrénécs.

Ilooker (British Flora) n'en fait point mention. Boissier n'en paule ni dans son voyage en Espagne, ni dans sa Flore d'Orient.

Linné (Species Plantarum Fd. III) l'indique « in Baldi, Austriae el Sibiriae Alpibus,» ce qui semblerait donner à notre plante la Sibérie pour limite seplentrionale.

D'un autre côté le Prodrome admel une Atragene Sibirica Mlill. inconnue à Linné, et ì laquelle il faudrait rapporter la lecalité du Species, si toutefois cette nouvelle espèce n'est point une simple variélé de l'Atragene alpina, ainsi que l'affirme Ledebours (Flora allaica).

En somme, il résulterait de toutes ces données que notre plante habite les Alpes de l'Emrope depuis les montagnes de l'Autriche et probablement même depuis la Sibérie jusqu'aux Pyrénées.

Les seules slations de cette belle plante, dans les limites de la Flore Suisse, seraient le pied de Salève, le le pied du Nôle, les Grisons, el une localitié des monlagnes du canton de Fribourg.

\section{Thalictrum minus L. Il saxatile Gaud.} Syn. Th. saxatile Schl. DG. Reut. Th. minus L. God. Rapin.

Celte espèce ou celte remarquable variété habite les 
rochers de Saleve, atr-dessus de Grevin, a droile de la Grande-Gorge. D'après Ilaller, ce Thalictrum est répandu sur les rochers des Alpes suisses. Suter, Hegetschweiler et Moritzi l'indiquent sur les montagnes. Murith signale plusicurs endroits du Valais. Gaudin mentionne le Valais, lo canton de Vaud et Salève. liapin el Godet citent le Jura el Salève. Grenier (Flore jurassique) croit que Ie Thalictrum saxatile du Salève est diflérent dı Thalictrum sylvaticum Koch, qui comprend, suivant lui, les diverses formes que les auteurs jurassiques décrivent sous le nom de Thalicl'um saxatile.

Notre plante ressemble beaucoup au Thalictrunı minus L., mais sa tige est plus striéc, elle n'est pas cou verte de poussière glauque, elle est rougeàtre inférieurement, les folioles sont petites, coriaces, à trois lobes entiers, la panicule est plus raide, les fleurs sont droiles, portées sur des pélioles moins liches, les carpelles plus grands, plus ventrus, rétrécis en pointe ì leur base et non oblus comme dans le Thalictrum majus. (De Candolle, fl. fr. T. V. Gaudin, fl. helv. T. III.)

M. Grenier (fl. jurassique) dit que notre Thalictrum de Salève, élant mumi d'une souche stolonifère, se distingue par ce caractere du Th. majus Jaq., dont lat souche est grosse et entièrement dépourvue de stolons.

C'est une plante à étudier de nouveau et sur laquelle on n'a pas encore dit le dernier mot.

Le Prodrome assigne pour habitation au Th. saxatile Schl. les collines boisées et les montagnes de l'Europe centrale.

Linné (Sp. Plantar. Ed. III) caractérise ainsi son Thalictrum minus: "Thalictrum caule folioso, foliis sex partitis, foliolis caulinis aculis, panicula divaricata, floribus nutantihus. Diagnoscitur apicibus foliorum purpurascentibus et caulis nebula coerulescente."

Habitat in Europae pratis. 
Ni la description, ni l'habilation ne conviennent notre Thalichrum de Salève.

D'après Boissier (royage en Espagne), le Th. minus serait répandu dans loute l'Europe, depuis la Laponie jusqu'en Grèce. Le Prodrome lui donne pour habilittion les piturages montagneux de presque toule l'Europe el la Sibérie. Les auteurs franeais, italiens, allemands et anglais le mentiomnent lous, mais en lui donnant des stations variées, pàturages, plaines of rocher's qui doivent se rapporter aux différentes formes qui sont encore à débrouiller.

Lspérons que II. Jordan pourra réussir ì élucider celte question.

\section{Helleborus viridis L.}

Cette espèce printanière se trouve assez alhondaminent dans les vergers de la commune de Per's, près de la Roche. Je la crois bien spontanée dans ecte station.

Gaudin la dit rare en Suisse; il signale Kiturg, Mendrisio, les rochers de la Chetelaz, Donio-d'Ossolit, Lugano et le mont Generoso. Iergetsehweiler l'indique au canton de Zurich. Morizi dorine un grand nombre de localités et la Roche en Savoie.

I'après 1. Godet, elle ne parait pas indigène dans te Jura, mais elle proviendrait d'anciennes cultures, au moins dans les localités citées.

11. Rapin la signale comme abondante dans les prés et les buissons, sur les coteaux de Saint-Lamrent et att-dessus de la Roche en Savoie.

Suivant Allioni, elte est fréquente en Piémont. Koch l'indique ega el là en Suisse et dans l'Allemagne centrale et méridionale.

Hooker la mentionne dans les buissons et les bois a sol calcaire de l'Ecosse.

Les auteurs francaisssignalent les $\Lambda$ pes du Dauphine, les Pyrénées, l’Ouest et le centre, l'Alsace, elc. 
Il'après Linné, " Ilabilat in montibus Viennensibus, Eugancis. 》)

II en résulte que l'Ilelleborus viridis $L$. se trouve dans toute l'Europé, sauf' dams la zone septentrionate.

\section{Nigella arvensis L.}

Plante erratique trouvée parfois dans les moissons de Reignier et d'Arenthon en Savoie (Reuter).

II. Rapin ne la mentionne pas.

II. Godet l'indique comme accidentelle of naturalisée dans quelques localités de sa Flore du Jura. M. Cirenier la signale comme abondante sur l'alluvion du Doulse, it Montbéliard. Gaudin l'indique comme rare atu-dessus de Côme el de Lugano.

Jlegetsehweiler ne prarle que des champs du Jurit, en ajoutant Schaffhouse et Zurich. Elle no parait pals exister en Angleterre. Les auteur's francais la signalent dans les moissons de presque tonte ba France.

D’après Allioni, clle est abondante én Piémont.

koch el Reichenbach l'indiquent dans les champs sablonneux et calcaires de l'Allemagne.

Linné lui assigne pour habitation les champss de l'Allemagne, de la France et de l'Italie.

Le Nigella arrensis L. rst done une plante qui se plait dans les cultures et surtout dans les sols caleaires et argileux; clle parail aceidentellement dans certaines localités, snivant lat provenance des senis, mais alle scmble bien naturalisée dans presque tous les champs de l'Europe centrale et méridionale.

Barbarea arcuata Ficich. Andr.

Syn. : Barbarca Taurica DC. Prodr. Erysimum arcuatum Presl. B. vulgaris R. Br. $\beta$ arcuata Gren. Fl. Jur.

Celte espèce, nouvelle pour la Flore de Salève, a été

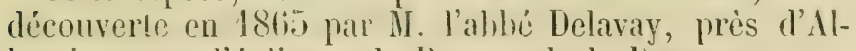
lonzier entre l'uglise et le bureau de la bouane. 
Nos auteurs suisses n'en font pas mention.

Rieichenbach et Koch l'indiquent gà et lí dans les localités humides du Nord, du centre et du Midi de l'Allemagne.

Grenier et Godron l'admettent et lui donnent pour habitation les hois el les lieux humides, sans citer de localités spéciales.

Tremeau de Rochebrune (cat. raisonné des plantes de: la Charente) signale une seule place de ce défritcment.

II. C. Des Moulins l'indique en Dordogne. De Ponzolz (II. du Gard) l'admet comme var. a du B. vulgaris R. Br. à Nìmes, le long des fossés.

Elle ne parait exister ni dans les Pyrénées, ni dans l'Guest, ni dans le centre de la France, ni en Anglelerre.

Le Prodrome l'indique dans les montagnes de la Tauric et du Cancase, sons le nom de D. Taurica.

En résumé, cette espèce présente des doutes. Ne possédant pas des échantillons de Salève, je ne puis pas affirmer si la plante trouvée par M. l'abbé Delavay est bien celle de Reichenbach qui parait différer du B. Taurica du Prodrome.

Les caracières qui distinguent le B. arcuata, du B. vulgaris se tirent de la forme des feuilles et surtont. des siliques qui, dans la première, sont arquées el huit lois plus longres que le pédoncule horizontal, landis que dans la seconde elles ne sont point arquées et ne sont que six fois plus longues que le pédoncule droit.

\section{Turritis glabra L.}

Syn. Arabis perfoliata Lam. Gaud.

Celle espèce a été trouvéc en petite quantité près de l'Mbbaye de Pommier et dans le vallon des Usses.

Gaudin cite un certain nombre de localités en Suisse, surtout les environs d'Aigle et le Bas-Valais où elle est commune. 
Hegeosehweiler l'indique au Jura, en Argovie vl dans le canton de Zurich.

Godet la donne comme assez répandue dans loul le domaine jurassigue, eependant je ne crois pas qu'elle ait été lrouvée dans la partie du lura qui nous avoisine.

Celte espéee existe en Allemagne, dans le nord, en Ecosse, en Angleterre, en Piémont el dans toute lat France, elle habite les bois montuenx, les collines pierreuses et les endroits exposés au soleil.

\section{Arabis hybrida Rent.}

Cette forme, décrite et figurée par M. Renter dans te supplément de lit fre Ed. de son calalogue, est une hybride des $\Lambda$. stricta IIuds. et muralis Bertol. Elle est toujours rare et je ne crois qu'on l'ait trouvée aill'nu's qu'au Pas-de-l'Echelle et autour des carrières de Veyrier. On pourrait cependant la découvrir an Fort-de-l'Ecluse et dans d'autres localités où croissent les deux parents.

\section{Sisymbrium acutangulum DC. Prodr.}

Syn. : S. Austriacum Jacq. $\gamma$ acutangulum koch.

S. pyrenaeum Vill. non L. Sinapis pyrenaica $L$.

Cetle espèce se trouve sur les rocher's de Salève, au Coin, au-dessus du Pas-de-l'Echelle et sur les assises dı Grand-Salève. D'après Gaudin elle est rare en Suisse, il l'indique à Salève, en Valais, dans le Val d'Aoste. Ruichenbach et Koch signatent plusieurs localités de l'Allemagne, Lederbours (fl. Altaica) n'en fait pas mention. Elle n'existe pas en Angleterre.

Les auteurs français l'indiquent dans les Alpes du Dauphiné, de la Provence et aux Pyrénées. Elle croit dans les Alpes du Piémont el au Mont-Cenis, où elle est abondante.

\section{Sisymbrium Sophia L.}

\section{Syn. S. parviflorum Lam.}

On trouve quelques maigres échantillons de cette plante sous les voùtes du Pelit-Salève, seule localité 
pour nos environs. D'après Gaudin, elle n'est pas rare en Suisse, où elle habite les lieux incultes et stériles; il l'indique a Berne, Avenches, Payerne, Neuchàtel, Bile ei Sion. Elle est rès-commune dans la plaine du Bas. Valais (Mlurilh). Je ne crois pas qu'on l'ait retrouvée à Nyon, el elle ne paraît pas exister dans notre Jura.

Ledehours (FI. alt.) l'indique dans les décombres et les endroits sees et un peu salins.

Suivant Reichenbath et Koch, elle croit le long des chemins, des murs et dans les champs sablonneux de l'Allemagne. Eile est commune on P'iémout ef en Angleterre et se trouve dans toute la France.

\section{Polygala alpestris Reich.}

Syn. P. amara L. $\gamma$ alpestris Koch. P. amara L. $\beta$ alpina DG. Gaud.

Cidte espece a été trourée sur les r'itons de Salèce pail MNI. Chavin et Riapin; clle est plus ahondante sur les sommités du Brizon et du Vergy el sur les pillurages élevés de notre Jura.

Gaulin l'indique sur le Jura, dans les Alpes de Bex ol il la Furka. Morizi ajoute la Gemmi el les Grisons.

D'après Koch, elle habite les haules Mlpes. Reichenbach ire parle que des Alpes do. Bex. Grenier at Godron ne la mentionnent pas dans leur Flore de France, mais M. Grenier l'admet dans sil Flore de la Chaine juras. sique.

Celle espèce se distingue de ses congénères palt une souche ligneuse et robuste, des liges nombreuses filiformes, étilées-ascendantes, sonvent nues inférieureinent ef munies à la partie supérieure de feuilles elliptiques et éparses, desfleurs réunies en grappes courles, de couleur bleue, varice de blane, an nombre de 10 ì 20 , des ailes ovales it nervures rimeuses a peine anastomosées, quelquefois entièrement libres. 


\section{Arenaria grandiflora L.}

Syn. A. mixta. Lapeyr. d'après le Prodr. A. Juniperina.

Vill. d'après Koch. Stellaria aculeala Scop. d'après Allioni.

Cette espèce, rare en Suisse, se trouve dans les éboulis rocailleux de Salève, an Coin, et près de la GrandeGorge, l'ancienne localité au-dessus du chiteau de Monetier est détruite. Elle ne parait pas se rencontrer dans notre Jura, bien qu'elle existe abondamment au Chasseron, au Chasseral et au Suchet.

IIaller, Suter, Gaudin, IIegetschweiler et Moritzi ne l'indiquent qu'à Salève et sur quelques sommets du Jura.

Koch et Reichenbach, outre les localités précitées, signalent plusieurs endroits do l'Allemagne, de la Croalie, de la Dalmatie et de la Pologne.

Allioni mentionne le Mont-Cenis et les Vallées validoises du Piémont.

D'après les autcurs français, on la trouve dans les $\Lambda$ pes, les montagnes du Dauphiné, la Chaîne des Pyrénées, le Languedoc et Fontainebleau.

Boissier l'a trouvée en Espagne sur les rochers onnbragés de la Sierra Nevada; il lui assigne pour habilation l'Espagne, les Pyrénées, la France méridionale ol centrale, la Noravie, la Carniole, les monlagnes de l'Italie et celles de la Sicile.

P.-S. J'apprends que M. J.-C. Ducommun a trouvé l'Arenaria grandiflora dans les rocailles du pied du Jura, autour du Fort-de-l'Ecluse.

\section{Ononis rotundifolia L.}

Cette belle légumineuse est assez abondante sur les talus d'éboulements au-dessus de Grevin et sous les voùtes supérieures du Pelit-Saléve. Elle paraît nulle dans la chaîne du dura.

J. Bauhin, liay et IIaller menlionnent déjà Salève, 
et l'illustre auteur de l'histoire des plantes suisses énumère plusieur's localités du Valais. Murith, Suter, Gaudin, IJegretschweiler et Horilzi répìtent les mêmes indications avec plus de détails, en ajoutant les Grisons et le Tessin.

Linné se borne aux Alpes de la Suisse. Koch el Reichenbach indiquent les Alpes dı Tyrol, de la vallée d.- l'Inn, les Grisons, le Valais, les Mlpes du Piémont el celles de Vérone. Allioni la mentionne également.

Ledebour's n'en parle pas dans sa Flora Altaica, ni Boissier dans son voyage en Espagne.

Villars, de Candolle, de Lapeyrouse, Grenier et Godron signalent les Alpes du Dauphiné, les Cévennes et les Pyrénées.

Elle n'existe pas dans l'Ouest, le Centre et le Nord de la France, ni en Angleterre.

II résulte de ces données que les Pyrénées scraient l'habitation la plus méridionale de cette belle espèce, qui s'étendrait delì, en paszant par les Alpes, jusqu’an Tyrol, sa limite septentrionale.

\section{Evonymus latifolius Scop.}

Cet arbuste, nouveau pour la Flore de Salève, a été déconvert par M. l'abbé Delavay, au-dessus de l'église d'Allonzier. liare en Suisse, il n'exis'e pas dans le Jura, et n'a été signalé que dans quelques localités d'liri el des Grisons, au lighi, ctc. On le trouve en Autriche et dans l'Allemagne méridionale. En France, il est indiqué dans les montagnes du Dauphiné et en Provence. Il ne parait pas croitre en Angleterre. En somme, il est beaucoup plus rare que l'Evonymus Europaeus L., et sa présence à Salève est une bonne fortune pour les amateurs.

\section{Vicia sylvatica L.}

Celle espèce se trouve dans les taillis du pied el de la région moyenne de Salève, au-dessus de Poummier, d'Archamp, an sentier de la Croisente all-ilessus de 
Monetier. On la retrouve aux Voirons et au MontSaxonnet. Elle n'existe pas dans notre Jura.

Nos auteurs suisses indiquent plusieur's localités, surtout dans les montagnes; elle sé trouve dans tout l'Allemagne, mais elle est rare dans le Nord.

Allioni la signale au bourg Saint-Maurice, à Fénestrelle et en Tarentaise (Brides et Pralognan), oủ jo l'ai récoltée. Elle habite l'Ecosse, l'Angleterre, le pays de Galles et l'Irlande.

Les auteurs français citent les montagnes du Dauphiné, la Grande-Chartreuse, les Alpes de la Provence, les Pyrénées et la Corse. Enfin Linné lui donne comme habitation les bois de la Suède, de l'Allemagne et de la France.

\section{Cerasus Padus DC.}

Synon. : Prunus Padus L.

Cet arbuste a fleurs odorantes se trouve en petite quantilé près de Reignier et sur quelques points du canton, où il a peut-être été introduit. Disséminé dans les vallées du Jura neuchàtelois et du ver'sant français, il ne paraît pas exister dans la partie qui nous avoisine.

Suivant Gaudin on le rencontre dans les cantons de Zurich, d'Argovic, de Berne, de Vaud, du Valais, et je l'ai cueilli aux bains de Louësche. Il habite le nord et le centre de la France et manque dans l'ouest et le midi. N1. Boissier ne le mentionne pas dans son royage en Espagne. Il est fréquent dans le nord de l'Angleterre. On le trouve en Allemagne, en Sibérie et en Piésnont. Il est donc disséminé dans toute l'Europe, sauf dans le Midi.

\section{Geum montanum L.}

Cette espèce alpine a été découverte près du premier Piton, par M. Bernett. On l'avait trouvée au Creuxdu-Vent d'où elle a ilisparu; clle existe dans les rochers du Colombier de Gex, senle localité pour le Jura (Michalet). 
Commune dans les pâturages des Alpes, on la trouve en Allemagne, en Suisse, en France (Damphiné, Auvergne, Cantal rt Pyrénées). Elle n’existe pas en Angleterre.

\section{Potentilla rupestris $\mathrm{L}$.}

Celte espèce se trouve en pelite quantité sur le PelilSaleve, an-dlessus du chiteau de Moneticr; elle est plus aloondante dans la plaine au bois de Bay. Elle est nulle dans la chaine du Jura qui nous avoisine.

Les auteurs Suisses la signalent à Genève, en Valais, dans les Grisons et le Tessin; nous en avons récolté de magnifirque pieds all-dessous de Salvan en descendant vers le Trient. Elle se trouve sur les rochers ot les endroils pierreux de l'Allemagne du centre el du midi, dans les $\Lambda$ pes de la Sibérie; elle est rare en Angleterre el commune en France, dont elle occupe les Vosges, l'Alsace, l'Auvergne, le Dauphiné, les P'yrénées et les monlagnes de la Corse; on la trouve en Piémont, en Espagne el, en résumé, elle habite l'burope depuis la Suède el la Sibérie jusqu’aux montagnes de l'Espagne.

\section{Potentilla alba L.}

Celte jolic plante se trouve sous les chathigniers entre Etrembieres el Mlornex; ses principales stations pour Gienève sont le bois de Bay et le bois de Prangins. Elle n'est pas dans lat chaine de notre Jura. Rare en Suisse, clle n'est indignée en outre qu'aux Grisons et dans le Tessin. Elle existe en Allemagne, mais n'y est pas commune, on ne l'a signalée en Angleterre que dans le pays de Galles. En France, elle habite l'Alsace? - Ies Alpes du Dauphiné? - la Provence el les Pyrénées. In somme, clle est répandue sur l'Europe centrale et méridionale, mais elle ne paraît commune nulle part.

\section{Potentilla caulescens L.}

Celfe espèce qui appartient plutôt anx Nlpes croît dans les fissures des rochers du vallon des Usses, du 
Môle el du Brizon; clle existe en petite quantié sur les rochers du Jura central, mais non sur la partie qui nous avoisine. On la trouve sur les rochers lien exposés de la plaine Suisse et surtout de la région subalpine. (Cantons de Vand, du Valais, des Grisons, de Lucerne, de Saint-Gall, ete.). Elle occupe en Allemagne toute la chaine des Nipes.

En France, elle est signalée sur les Alpes du Dauphiné, les Cévennes et les Pyrénées; elle se trouve aussi en Piémont.

M. Boissier l'a récoltée sur les rochers calcaires de la région alpine de l'Espagne.

On ne la trouve ni dans le. Nord de l'Europe, ni en Angleterre.

Elle habite les Alpes de l'Europe centrale el méridionale, les montagnes de l'Italie, les Pyrénées et les montagnes de l'Espagne.

\section{Potentilla petiolulata Gaud.}

Syn. : P. caulescens L. $\beta$ peliolulosa Ser.

Colte espèce on celle variété n'a été trouvée que dans les fentes des rochers à Salève, sur les bords du Fier, à Chambéry et à Saint-Triphon. Elle doit se rencontrer ailleurs. On la distingue de l'espèce précédente par ses folioles pétiolulées, par ses pétales largement obovés et par son réceptacle très-velu.

\section{Rubus collinus DC.}

Cette ronce a élé découverte par M. Heuter sur Irs rochers du Petit-Salève entre Murnex ot Monelier, sur le versant oriental.

M. Rapin l'a trowríc en outre il Joux-Brìlíc audessus de Branson:

M. Godet ne l'admet que comme var. $\beta$ du Rubus discolor W. el N.; les autres anteurs suisses ne la mentionnent pas.

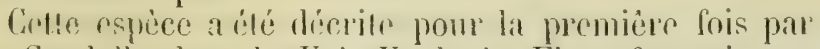
De Candolle dans le: Vol. V. de la Flori franease ef 
indiquée an pied du Pic Saint-Loup, près de Montpellier. Grenier et Godron la signalent sur les coteaux arides des Vosges, des Cévennes, de l'Ardèche, de la Provence, du Languedoc, des Pyrénées et de la Corse. Boreau l'admet dans sa Flore du rentre de la liance el indique piusieurs localités Elle se distinguc par la forme el la nature coriace de ses feuilles recouvertes en-dessus d'un épais duva blanchàtre qui s'étend sur les rameaux of sur les calices. Le fruit est formé de carpelles gros ot peu nombreux. Dans la variété hybride. simnalée par llercier, Ies fruits sont tonjours arortés.

C'est une espèce plutot méridionale qui s'élève jusque vers Nancy.

\section{Rosa vestita God.}

Syn. : R. Spinulifolia II $\mathrm{rm}$. $\beta$ restila Rap. R. Inmontosa var. scaliriuscula Ser. Prodr.

Ce rosin se rapproche beaucoup du R. spinulifolia, dont il diflère par sos fruilles petites el rowvertes d'un duxw grisilte ; Ies fleurs sont plus pailes et le tube du calier est oblong hispide; les aignillons sont rares of droits. II a été déconverl par M. Lienter sur la pente du Grand-Sialève qui dominr le vallon de Monelier. II en rxiste plusirurs pieds sur les talus d'éboulenents an-dessus d'Archamp; il a ite tronvé ans Voirons of dans le Jura neuchâtelois.

\section{Rosa Salaevensis Rap.}

Celle belle repece a été lécrite par M. Rapin daus les Bulletins de la Soriétó Mallérienne. Elle se trouve dans Ios taillis ath-dessus d'Arehamp, an chemin de la Croisette el an-dessous des Treize-Arbres. On l'a reIrouvée dans lo dura nenchitelois.

Cie rosier se reconnait à ses tiges élevées, colorées en rouge, munies d'aiguillons rares, droits, longs a subulés. Les fleurs sont grandes el d'un beau rose, les pédoncules el les calices glabres on hispides-glanduleux. Les fruis sont oblongs, précoces el couronnis par les sépales dressés et persislanls. 


\section{Rosa Sabauda Rap.}

Ce rosier, découvert et décrit par M. Rapin, se rapprochr du R. pimpinellifolia, dont il diffère par ses aiguillons robustes ef comprimés, ses fleurs rosérs or sers firuis précoces el ronges. Il se retrouvera probatblement sur le calcaire jumassique; mais, jusqu'i yrésnnt, il u'a été signalé que sur le sommet de Salève.

Rosa Reuteri Godet, Reut. cat.

Syn. : R. rubrifolia $\beta$ Reuteri God. R. glauca Vill.

Voici ce qu'écrit M. Godel dans sa Flore du Jura, p. 208, a propos du li. rubrifolia Vill. $\beta$ Renueri : "La var. $\beta$ se distinguera peut-être plus tard comme espèce quand elle aura été mieux étudiée, et prendra alors le nom de $\mathrm{R}$ Reuteri. ”

Ce rosier est commun à Salève et au Bôle, près de la Dôle.

Il se distingue par ses feuilles glanques, plus obtuses ef moins rouges que eelles du rubrifolia, ses stipules grandes et foliacées, ses pétales plus longs que le calice, larges et se recouvrant par les côtés, d'un rose vif; ses fruits gros, ovö̈des, d'un rouge orangé, précoces, à sépales calucs au commencement de la maturité.

Rosa montana Chaix in Vill.

Syn.: R. Reynieri Ilall. fils. D'après Gaudin, Reulor. R. rubrifolia II montana Gaud. D'apres lionler, Godet.

R. glandulosa Bell. Koch. D'après Reuler, Cionlu!, Rapin.

P. rubrifolia G glumdulosa Ser. in Prodr. DC. D'apris Gaudin.

Koch décrit le Rosa glamdulosu Bellardi en lui assignant comme synonymes: IR. Reynieri IIall. fils. R. glabrata Vest. R. Montana Murith non Villars. P. rubrifolia II montana Gaud. R. rubrifolia ל glandulosa Ser. in D)G. Prodr. Il ajoule, en parlant du R. montana Villars: "Slylos elongatos commalos babet, 


\section{4}

ul Rosa arvensis. " Or, Villars se borne à dire: "Elle a ses pistils allongés et velus eomme le R. alvensis L., mais son trone se soutient et ne sallonge jamais aulant; d'ailleurs elles se ressemblent assez. " Après sa description du R. arensis L., Villars ajoute: "Pon' distinguer la précrdonte (R. montana) de cotle espece (Ii. arvensis), il laut savoir que erlle-ci no vient. que dans les plaines et celle-li sur les montagnes.... Elles sont l'une et l'autre it fleurs blanches, phus pelites ainsi fue le fruil ef lo trone, mais l'une se sonlient rt l'aule est rampante. Les olservateurs jugeront du mérite de ces différenenes. s) (Vill. Damph. Toi. III, p. 5/4, 548, 549.) II res vai yue Villars, dans la descriplion du R. arvensis L., ne parle en aucune lacon des pistils.

Willdenow (C. Linn. Sp. Plant. Ed. IV. Berol. 1797) ne parle pas de la sombure des styles du R. arrensis L. il dil: "Stylis pubescentibus longiludine staminum, " et ailleurs : "stylis clongalis. "Dans celte édilion, il adrect Ie R. Montana Vill. dont il domue une description.

Lamarck (1l. fr. Ed. 2. an. III de la Rep.) no parle point des styles soudús en colonne du R. arvensis. Latmarck of de Candolle (synops. plantar. in fl. gallic. descript. 1806) caractérisent amsi lo R. arvensis : "Slylis in colummam cylindricum glabram coulitis, stigmatibus distinctis, caule repente. ")

De Candoll: (fl. fr. 3. rd. 1815) en décrivant le R. arrensis L. dit: "Les priales of los etamines sont insérés sur le bord d'un disque charnu formé par la soudure naturelle de tous les styles: du milieu de ce disfure s'élive une petite colonne glabre qui s'épanonit all sommet en plusiours sligmates distincls. "

Dans le Ve vol. de celle même édilion, de Candolle domo la description du R. montana lihaix in Villars et du R. Glandulosa Bellart. qu’il admet comme espèces distincles.

Seringe (Prodr. DC. T. II. 1825) natmet ni le P. montana Vill. ni le R. glanmblosa Bell., il les cile 
conme synonymes du Ri. rubrifolia Vill. of glandulosa, Ser.

I)uby (Bot. Gallic. ed. 2. 1828) admet et déerit le Ii. glandulosa Bell. el n'a pas le H. monlana Vill. Seulement à propos du li. Iomentosa Sm. il admet la varićte $\beta$ scabriuscula Ser. a laquelle il altribue comme synonyme le R. montana fl. fr. T. 5. p. 539, non Villars.

Loiseleur Deslongchamys (11. Gall. éd 2. 1828) fail du R. glandulosa Bell. la variélé $\beta$ du R. rubrifolia Vill. Quant au IR. montana Vill., il le donne comme synonyme de son R. monlana Lois. (nouv. Duham. 7. 1). 8) en ajoulant: "An satis distincta a Prosa Canina."

Mutel (11. du Dauphiné 1830) adunet le Ih. montinat Vill. et lait du R. grtindulosa Bell. la var. $\beta$ du li. rubrifolia Vill. DC.

Mulel (11. fr. 1834) a vu l'échantillon unique de l'herbier de Villars it la Bibliohnégue de Grenohle, l'étiquetle porte en note: "Frutex R. caninate magnitudine, rami purpurascentes, pelala alba purpurea; magis accedit ad. R. villosam. )

Mutal ajoute que le Ii. montana Vill. diffère du li. glandulosa Bell. (Syn. Ii. monlana Schl.) frat le fruil lisse, les aiguillons de la lige tres-grètes el le calice non glanduleux.

licichenbach (11. Gorm. excurs. 1833 ) considere le R. ylandulosa Brll. qu'il admet, comme un hybride des R. rubrifolia el villosa, puisqu'il le caractérise de R. mbrifolio-villosa, il donne comme synonymes: li lieynirri Ilall. fil. et li. montana Schl. Vill. avecdoute. Il donne comme localités la Suisse, Salève, le Frioul, le Mont-Cenis et le Steyermark.

Grenier et Godron (fl. de Fr. 1848) athuchent ed décrivent le R. montana Chais, in Vill., en donnant cornme synonymes: R. rubrifolia II montana Gaud. el li. Glandulosa Bell.

Grenicr (11. de la Chaine jurassique 1865) donne le R. glandulosa Bell. comme synonyme du IR. alpina L.; 
il se base principalement sul ce que bertoloni (fl ilat. 5. p. 210) a consiaté que te R. glandulosa Bell. est un R. alpina et sur ce que II. Rapin a vu dans l'herbier de de Candolle un échantillon du R. glindulosa étiqueté de lat maison de Bellardi, et qui ne serail qu'un R. alpina.

$1 \mathrm{l}$ admet le R. montana Chaix in Vill, en l'indiquant à Saleve, et ajoutant: "Il ne parait pas encore avoir été trouvé sur notre chaine jurassique proprement dite. )

Il résulte de toutes ces cilations que les auteurs sont loin d'être d'accord quant à la synonymie. Les deseriptions présentent la nème divergenee ct il est difficile de: se reconnaitre an milien de ce conflit d'opinions.

Linsi pour la taille les uns lui dounent de 2 a 3 picds, l'autres de 1 à 2 mètres.

Guant aux aiguillons, te plus grand nombre sont d'accord pour les considérer comme lares, minces, mriles, presque droits a peu recourbés; cependant quelques-uns les disent arqués.

Pour les stipules, la plupart n'en parlent pas, los uns les décrivent comme planes elliptiques allongées, it oreilles aigues et dressée: ylabres; d'autres discnt qu'elles sont grandes, colories, oblongues, cuneilormes avee des oreilletles ovales, divergentes, largement lancéolées à bord déntelé el glanduleux.

Pour les feuilles, presque tous admettent de 5 a 7 folioles (la Fl: Fr. 7 à 9).

Les folioles sont pour tous petiles, courtes, rondes, ovales ou ovales arrondies, ou ovales elliptiques, $\mathrm{Ol}^{\prime}-$ dinairement obtuses.

Pour la plupaut, elles sont doublement dentées, en seie a dents aigues, un peu convergentes all sommel u. ciliées glanduleuses; - jour quelques-uns, elles sont. simplement dentées, glabres et vertes en dessus, glitbres en dessous, ou bien lavées de pourpre, très-glauques en dessous arec des villosités et des glandes sessiles très-petites.

P'our lous, les petioles sont minces, hérissés de soies 


\section{7}

romeres, glamdulense's et d'atignillons rates el miners.

Pour lons, les pédoncules son! hispides, hérissís de: poils glanduleux, presque ípineux, ordinatrement solilaires, droils, s'allongeant après la floraison.

Pour lous, le tube du calice est ovoide oblong, hirissc de longs poils spiniformes et glanduleux.

Les sépales sont pour les uns peu découpés, indivisés, dressés et persistants, on devenant cadocs apres la malurité, un peu phus courts que la corolle, glanduleux velus; pour d'aulres, Ies divisions calicinales sonl longues, lerminess par un appendice lancéolé, sourent pinnalilides, plus longues yue la corolle, d'ahord étalées, puis redressćes, persistantes et comronniml le fruit à la maturité.

les fruits sont, pour la plupart, gros, ovö̈des, venIrus, oblongs, puelquefois un peu étranglis an sommet, l'ecourerts de poils glanduleux, précoees; d'après qurlyues-uns, ils sont glohuleux, pelits, rouges et glubres.

La corolle est hlanche suivant les uns, rouge suivant l's antres; les pétales sont échancrées en forme di" corur; les fleurs sont grandes, solitaires, terminales, longucment pédonculées d'un rose pàle, ou hien elles sont pelites, quelquefois réunies en corymbe au nombre de deux ou trois.

Pour s'en tirer : all milien de toutes ces divergences, i) lamdrait voir sur place la plante derenge par Chaix el li comparer avec celle que nous prenons pour le li. montana.

Du reste, cel arbrisseau parait très-raro parlout ou il a été sionalé; a Saleve, je n'en comais que quelques pieds, et sauf les localités comnues en Suisse, dans l'Allomagne méridionale, en Daujhiné et en Piémont, il manque dans le Jura et dans tout le reste de la France. Il ost vai que de Lapeyrouse l'indique aux Pyrénćes (T. 2 suppl. p. 60), mais M. Philiplye n'on fait pas mention dans sa Flore les Pyrénées. Boissier (voyage en Espagne) n'en parle pas non plus.

La rareté de celte plante et les contradictions qui existent dans les descriptions des auteurs, me portent 
a me ranger i l'opinion de Reichenbach et i considérer ectle forme comme une hybride du R. Iubrifolia avec l'alpina ou le pimpinellifolia.

Il faudra done l'étudier de nouveau, s'assurer si les carpelles donnent des graines qui puissent se reproduire et faire des essais de semis.

Guoi qu'il en soil, nous avons à Salève la plante décrite par Gaudin, Godet, liculer et Riapin; l'ste it savoir si c'est la même que celle du Dauphiné, car les deseriptions de Chaix el de Villars ne concordent pas avec celles de nos auteurs et paraissent se rapporter it une toule autre espèce.

\section{Scleranthus perennis L.}

Celte plante ne se trouve que sur les couches sidérolitiques de Salève, au-dessus de Cruseilles. On la rencontre aussi dans le sable molissique des Voirons, au-dessus de Bonne. Elle n'existe pas sur le calcaire jurassique.

Giudin et les auteurs suisses l'indiquent dans des endroits sablonneux ì Bìle, au Valais, dans le canton de Vaud et aux Grisons.

Koch el Reichenbach lui dounent pour habilation les champs sablonneux el les paiturages secs de l'Allcmagne.

Allioni dit qu'elle est commune dans la vallée de Fénestrelle.

Les auteurs francais signalent les terrains siliceux de la plus grande partie de la France.

Elle se trouve en Angleterre.

Le Prodrome lui assigne pour habitation les champs stériles de l'Europe et de l'Orient.

\section{Sedum villosum L.}

Lette plante des termains granitiques ne se troure (qu'au bord d'une petite mare creusée dans lo salle cristallin, au-dessus de ta Groisette. Eille ne parait pas exister dans les tourbières de notre Jura. Un la rencontre dans plusicurs localités de la Suisse, soil de la 
Mlaine, soil des Alpes. (Ciantons d'Argovie, Einsiedlen, Glaris, Uri, Vaud, Valais, Grisons, etc.) Nons l'arons récoltée sur les prairies humides du plateau du Simplon, où elle est très-abondante. La forme allongée se trouve dans les parties basses, la forme courte sur les hautes Alpes jusqu’à 7,000 pieds.

Les auteur's allemands, ilaliens, franeais et anglais, la signalent tous, et en résumé elle habite la suède, lit Litponic, l'Ecosse, l'Angleter're, l'Allemagne, la Suiss', le Piémont, la France el l'Espagne, prélérant les terrains humides et siliceux.

\section{Sedum anopetalum DC.}

Syn. : S. Ilispanicum DC. non L., S. rupestre Vill. non L. Prodr.

Gelte espèce, voisine du Sedum refexum L., se plail dans les rocailles seches el chaudes du Pas-de-l'tichelle et du Coin. Ce Sedum se reconnaìt à ses pétales d'un jaune pâle, redressés pendant la floraison et non pas ćlalés comme ceux du Sedum reflexum, avec la variété glaucum duquel on peut le confondre.

II n'a élé trouvé, en outre, que dans le canton do Vaud el dans le val d'Anzasca. Pour l'Allemague, il n'est indiqué que sur les rochers et les murs prés de: Trieste. Les auteurs francais ne le siognalent que dars le Midi de la France, d'où il remonte jusqu'ì Genève.

\section{Myrrhis odorata Scop.}

Syn. : Scandix odorata L., Chaerophyllum odoratum Jacq.

Cette plante, souvent cultivée poln ses propriétés aromatiques, et qu'on trouve toujour's autour des ruines des anciens cumvents, par exemple aux Voirons, parail cependant bien spontanée à Saléve, au-dessous du Grand-Piton. Dans le Jura, elle ne se rencontre que dans le voisinage des habitations; elle est commune dans les pâturages du Brizon. 
On la trouve dans les prairies alpines el subalpines ale la Suisse méridionale, dams les Vosges, dans la chaine des Apes de l'Allemagne du Surl, du Pićmont, diuns les monlagnes de l'Ecosse et du Nord d'Angleterre, du Daıphiné, dans les Pyrénées, etc.

It en résult: qu'clle habile l'Europe centrale el méridionale depuis l'Espagne jusqu'n Isie mineure; mais elle a été introduile dans beancoup des localités où elle est indiquée.

\section{Galium spurium L. y tenerum Gir. \& G.} Syn. : G. lenermm Sehl. Gitud. G, aparine L. vall. lenerum Schl. Koch.

Celle variété se tronve sous les voules supéricures du l'etit-salive; clle est mulle dams le Jura et se rofromve sur les rochers cavernoux des Alpes de bex, sur lat Gemmi el dans lat vallée de Zermatt. Lat forme grêle ef rédaile de cette plante est due probablement it son habilation sous des rochers en saillie, catr on ne la rencontre pas aillours.

Le type occupe toule: l'Europe, la Mésopolamic. l'Asie septentrionale el l'Amérigue du Nord oit elle a élé introduite el naturalisée.

\section{Doronicum pardalianches L. Willd.}

Syun. : I. latifoliun Clus. D. Malmoli Tausch. Ricich.

Crelte belle espèce se trouve dans un potit bois pres do: lat Fontaine dite de Jules-César, sur la pente du GrandSalève yui domine Monelier et au-dessus d'Archaum. Nous ne l'avons jamais rencontrée dans la partie du Jura rapprochée de nous, hien qu' elle soit indiquée par llaller stl1 la montagne de Thoiry. Elle est assez rare en Suisse a n'ust signalée que sur quelques points du canton de Vaud, du Valais et des Grisons.

Elle est répandue depuis Ilambourg dans les vallóes dı Lhin, du Main, en Autriche et en Bohème; elle est abondante en Piémont et elle oceupe une grande partie de la France, l'Ecosse el le Nord de l'Angleterre. 
M. Boissier n'en parte pas dans un voyage en Espagne, mais il signale le Doronicum seorpioides Villd. qui a abo inliqué à Salève par Koch, mais que nous n'avons jamais rencontré.

Colui-ci se recommait à son rhizome court, horizonlal, nourux, sans stolons, ì sal tige ćpaisse, moins rameuse, it ses feuilles tronquées al la base al non cordiformes.

\section{Arnica montana L.}

Rare ì Salève, elle n’a été Ironvée qu'atu-dessus de Ciruseilles. Elle est égalmuent peu répandue dans le lura et ne parait pas cxister tans sa partie méridionale. Fréquente dans les pàlurages alpins de la Suisse, elle 'roit sur les montagnes de Berne, d'Uri, de Viatud du Valais, ete. En France, elle occupe surtout les monlagues de grès, de basalte cl de granit (Vosges Coted'Or, Pilat, Cantal, Monts-IJorer, Dauphiné, Pyrénées), certaines plaines sablonneuses et les landes près di: Dax.

Elle est abondante sur les Nlpes de la Savoie et du P'iémont.

Elle n'a pas été signalée en Angleterre.

En résumé, on la rencontre depuis le hord de lat mer jusque sur les paturages les plus chevis pris des neiges éternelles.

\section{Serratula nudicaulis DC.}

sin. : Centaurea nudicaulis L., Calcitrapa nudicaulis: Lam., Carduus cerinthoides Willd. C. cerinthatolius Vill.

Cotte rare espèce ne se trouve à Salève que sur les assises des grandes roches perpendiculaires d'Ar-hatmp. Gaudin est le premicr qui l'ait signaléc à Salère comme ayant étí trowvés en 1829 par M.1. Girorl "t Papin. Hès lors, tous nos auteurs ont donné la mème indication, à l'exception de Haller et de Suter. Elle ne eroit ni en Allemagne, ni en Angleterre. 
Pour la France, on ne la trouve que sur les Alpes du Dauphiné et en Provence sur une seule localite; clle manque dans les Pyrénées et se retrouve en lispagne.

D'après $\mathrm{II}$. Boissier, clle habite les rochers de formattion calcaire de la région alpine de la Sierra Nevada, ill-dessus du sommet Dornajo. Elle se trouve en outre en Catalogne, en Arrago:; au royaume de Grenade ct de Valence, en Italie, au Midi de la France, en Piémont et it Saleve qui est la limite septentrionale de notre plante.

\section{Scorzonera humilis L. Willd.}

Syn. : S. plantaginea ct S. macrorhiza Sehl. Gaud., S. nervosa el Poir. d'après le Prodrome.

Koch admet et décrit le S. humilis L. fl. suec. comme étant bien la vérilable espèce de Linné et il lui doune pour synonymes: S. lanata Schr., S. nervosil et Lam., S. angustifolia Wiggr., S. planlaginea Schl. Reich., S. microrhiza Schl. Gaud.

M. Rapin a trouvé récemment celle espèce dians un endroit marécageux au-dessus d'Archamp. Elle croit en outre dans les clairic̀res hrmides du bois de Cranves, all pied des Voirons, où elle est rare : clle a élé découverte par le docteur Mercier dans dea prés mouillés att-dessus de Coppet; elle est abondanie aut-dessus de la gare de Chancy, on allant i Farges, dans des low calités analogues (M. Brun).

Pour le Jura, on la trouve dans la vallée de Joux el près de Pontarlier, de Besançon, de Salins el d'Arhois (Godet).

Nos auteurs suisses la signalent en outre dans la vallée du Rhin, à Zurich el à Sarganz.

Ellu es répandue dans les prés tourbeux d'une grande partie de l'Nllemagne depuis la suède.

Elle est commune dans le Nord, l'Est, le centre, l'Ouest et la région méditerranéenne de la France.

Elle n'est pas indiquée en Angleterre. 


\section{Scorzonera austriaca Willd.}

Syn. : S. humilis Jacq. non L. d'après Koch. S. humilis L. $\beta$ austriaca DC. S. austriaca Gaud. d'après le Prodrome.

Cette espèce rare pour Salève, n’a éé lrouvée qu’andessus de Grevin et d'Archamp, en pelite quanlití. Elle vient aussi sur le Vuache, mais ne paraît pas exisler sur le Jura.

En Suisse, clle croît sur les rochers du canton de. Vand, à Bex el Olon, dans le Bas-Valais (it SaintMaurice, Saxon, Saint-Léonard) où alle est abondante.

On la trouve dans l'Allemagne méridionale, en Autriche, en Istrie.

En France, on indique la Côte-d'Or, les rochers de Fontaincbleau, le Sud de la Loire, Ins Alpes du Dauphiné, le Languedoc.

Elle ne paraît pas sc trouver aux Pyrénées.

Cette espèce se reconnait à sa souche grosse, épaisse, profonde, couverte de fibres chevelues abondantes, formées par les nervures persistantes des feuilles. La lige esl monocépliale, assez courte, presque nue, glabre, un peu renfée sous la calathide unique. Les feuilles radicales sont ovales, larges, ondulées, quelquefois étroites-lanceolées (var. angustifolia Sat.). Les feuilles caulinaires sont au nombre de deux it quatre, étroites, squamiformes, presque nulles. Les languettes de la corolle égalent le tube du calice laineux au sommet. Les akènes sont striées.

Elle habite les rochers exposés an soleil.

Le Scorzonera humilis L. a la souche épaisse, dépourvue de fibres chevelues, mais entourée d'écailles scarieuses et noiratres, débris des anciennes feuilles.

La tige est monocéphale ou bifurquée et dicéphale, élevée, dressée, fistuleuse, d'abord lanugineuse, sır'tout au sommet, puis glabresçante.

Les feuilles radicales sont longues, lanceolées, acu- 
minées, pourvues de 5 à 7 nervures; les caulinaires sont peu nombreuses, petiles, linéaires et dressées.

La corolle est une fois plus longue que le périeline, a limbe égal au tube du calice pubescent.

Les akènes sont à côtes presque lisses et non striées.

Elle habite les prairies humides el tourbenses.

\section{Hieracium lanatum Vill.}

Syn. : Andryala lanala L., Ileracium tomentosum All.,

II. verbascifolium Pers.

Cortle belle espece se trouve on pelite quantité sur Ies grandes poches d'Archamp avec le Serratula nudiraulis; il croit également sur les rochers du pied du Mole, du coté de l'Onest. Rare en Suisse, ne se trouve qu'en Valais, à Saillon, Saxon, au-drssus de Brieg, en montant au simplon, aul Chrmin neuf el on allant it Loesche par la vieille route, entre Varen el Inden, où je l'ai récolté abondamment.

Il est indiqué en Piémont, en Dauphiné, en Provence, dans les Landes, mais it ne parait pas se tronver dans les Pyrénées.

\section{Hieracium andryaloides Vill.}

Syn. : II. tomentosum var. All. Antryala dentala IIpgetsch.

Cette plante rare croit sur les rochers du vallon de Monetier, pres du chatean de l'Ermitage el sur les assises supérieures du Grand-Salève. Il a été trouvé par M. Huet sur les rochers du Fier, près de Seyssel. Gaulin admet une var. $\beta$ pilosum qui se trouverait en Valais et dans le Tessin; le type, suivant lui, avait été trouvé at Salève par lo Rev. Ducros, Em. Thomas el M. Rapin. Il n'existe nulle part ailleurs en Suisse, ni en Allemagne.

Les auteurs français le signalent en Piémont, en Savoic et dans les montagnes du Dauphiné. Il ne croît pas dans les Pyrénées. 
Salève serait done la station la plus septentrionale de ce rare Hieracium.

Observ. Allioni avait réuni sous le nom de II. 10mentosum les denx espèces dont nous venons de nous ocenper. Elles se distinguent cpendani par des caraclères positifs tirés du porl, de la direction des ratmeaux, de l'indument, de la différener de grandeur des fleurs, etc., en sorte qu'on ne peut pas les confondre, surloul quand on les a examinées sur le vif; on en jugera d'après la description.

II. Ianatum Vill. Péricline à folioles cuspidées, dronssées avant la lloraison, couvert d'm duvet lainenx, épais, ordinairement dénudé à l'extrémilé; corolle glabre au sommel, pulvérnlente à l'extérienr; feuilles rarlicales épaisses, molles, courertes d'un duret laineux, ì peine rapprochées en rosetle, courtement pétiolées, oblongues, entières on sinueuses at quelquefois denlées i la base; feuilles caulinaires $1-5$, ovales, aiguës, parfois nulles; tigge de 1-3 décimètres, simple ou rameuse dès la base, rameaux ascendants et rapprochés, parcourus dans toute leur longueur par de petiles nervures saillantes et couvertes d'un duvet laineux ćpais, poils fortement plumeux et allongés.

II. andryaloides Vill. Péricline à folioles blanches lomenteuses, mais non laineuses, un peu recourbées avant la floraison; dents des corolles ciliées; feuilles radicales moins larges et profondément dentées à la base; feuilles caulinaires ordinairement pétiolées at denticulées à la lase; liges de 10 à 15 centimètres, simples ou rameuses, arrondies el non nerviées; poils de la tige et des feuilles moins géniculés, à barbes plus rapprochées, formant un duvet plus mince, plus appliqué, laissant apercevoir la couleur verle des fenilles.

\section{Hieracium glaucum All.}

Syn. : II. glaucum Willd. DC. Gaud., II. Saxatile Jaer.,

II. scorzonerifolium Vill., II. Allionii Moṇn. 
Co bran Ilieracium croì sur les rochers de Saleve, an Coin, an-dessus d'Archamp et vers la GrandeGorge; on le retroure au Hôle el au Brizon; assez. rare dans notre Jura, on le trouve cependant it la Dile, au Reculet el an Vuachr. (II. slaucum All. $\beta$ Juralense (ir. el G.) Il occupe plutòt la partie méridionale de la Suisse (Berne, Vaud, Valais, Grisons). On le trouve sur les rochers et le long dos torrents des Mlpes do la Bavière, de l'Autriche, du Tyrol, de la IIongrie, du Piémont, sur les montagnes du Danphinć. II parail nul dans les Pyrénées et en Anglelerre.

\section{Hieracium Pseudo-Gerinthe Koch.}

Syn. : II. cerinthoides Schl., Thom., Sul., IIegets.,

Vill., non L. Il. amplexicaule L. $\varepsilon$ psendo-cerinthe Gand.

Gette plante rare a été trouvéc par M. Bernetl sur les Pitons de Salève et sur les rochers entre la Grande el. la Pelite-Gorge. Elle est nulle sur le Iura, peu répandue en Suisse (Alpes du canton de Vaud), elle a élé signalée sur les montagnes du Dauphiné et sur celles de la Corse.

Cette espèce qui se rapproche beaucoup du R. amplexicaile $L_{\text {., }}$ s'en distingue par le collet de la racine poilu, la forme des feuilles peu dentérs, les styles jaunes of les rameaux moins nombreux, formant un corymbe peu serrí. On ne peut pas Iss confondre quand on compare des échantillons vivants.

Ce Ilieracium deviendra prohablement moins rare ì mesure qu'on le reconnaitra ef qu'on le distinguera du II. amplexicaule L. Ce dernier occupe les fentes des rochere de l'Allemagne, de l'Angleterre, du Piémont, de l'Iralic, de la France, de l'Espagne et du Portugal.

\section{Pyrola media Sw.}

Celte espèce rare ct bien distincte de la P. rotundifolia et de la P. minor, n’a été trouvée que dans une seule localité de Salève, daus les éhoulements au-rlessus d'Archamp, en compagnie arec l'Atragene alpina.

Nulle dlans le Jura of dans la France, elle n'ost in- 
diquér en Suisse que près de Rüggisherg el de Thun, au Righi, au pied du Pilate, entrer Kusnacht el Sireboden, et aux environs de Berne.

Elle est plus abondante en Allemagne (grand-duché de Baden, bords du Rhin, Mecklenburg, Mr'sse, Gottinpen, Save, Thuringe, Bohène, Barière, Tyrol, Silésie).

Elle est commune en Suède, en Ecosse, dans le Nord de l'Angleterre et en lilande.

Saleve serail done la station la plus méridionale de relte plante qui se plail dans los bois ol los endroits ombragés.

\section{Pyrola chlorantha Sw. •}

Syn. : P. virens Schw.

Calle espèce a été trouvér dans te bois do Barioz, près de la Caille, par II. l'abbé Puget. Eile croit Ggalement au pied du Jura, près de Coinsins, où elle a élé déconverte par. M. Rapin; du reste, clle est rare dans le Jura. Elle est signalée sur quelques points du canton de Vaud, de Berne, du Valais el de la vallée du Rhin. Elle est répandue ça et là dans toute l'Allcmagne, mais elle paraîl nulle en Angleterre.

En france, on la trouve dans les bois de la IIanteLoire, du Dauphiné, des Hantes-Alpes, etc.

Elle sétend depuis la Russie, la Suède, le Canada supérieur, jusqu'au Midi de la France.

\section{Gynoglossum montanum Lan.}

Syn. : C. sylvaticum Ilaenk, C. officinale $\gamma$ L., C. Dioscoridis Vill.

Se trouve à Salève, an pied des rocher's oi croit le? Serratula nudicaulis, et au-dessus de Pommier. Il existe sur plusieurs points du Jura, sauf dans la partic qui nous avoisine; dans le canton de: Vaud (an-dessus de Bex el d'aigle), dans le Valais, les Grisons ol prés de Wallenstart. 
En Aliemagne, il est disséminé sur plusieur's endroits (Autriche, Hanovre, Wurtemberg, Baden, dams la vallée. du Rhin, celle du Neckar), etc.

On le trouve en Ecosse, en Angleterre et en Irlande.

En France, il croil dans les Vosges, la Côte-d'Or, le Jura, le Dauphiné, les Pyrénées, les environs de l'atris, etc.

En résumé, il habite les bois monlueux de presque toute l'burope, s'étendant depuis te royaume de Na. ples et le Péloponèse jusqu'an Cancase at en Virginie.

\section{Primula Auricula L.}

J'ai récolté celle espèce alpine sur les rochers de la rive gauche du torrent des Usses, all-dessus des Bains de la Caille. Elle existe dans le jurat septentrional et unanque dans la partie qui nous avoisme. Elle occupe toute la chaine des Alpes suisses, ol la variété it llom's violettes se rencontre dams les firisons et les monlatgnes du Piémont. En franer, elle est commune dans les Alpes du Dauphiné el rare dans les Pyrinées; elle. manque en Angleterre.

Elle habile en outre les Apennins, l'Antriche, la Foret-Noire, Baden, la Bavière, la Styrie, les Carpatthes, la Sibérie, jusqu'au delí des limites do la Chine.

Gyclamen hederaefolium. Ait. non T'en. d'après Koch.

Syn.: C. Neapolitanum, Ten.

Ciefte rare espéce a été découverte par M. l'abbé Delavay, dans le vallon des Usses, au pied des rocher's qui dominent les Bains de la Caille.

Ce Cyclamen n'est comnu on Suisse qu'au-dessus de liocle (canton de Vaud) el dans le Tessin. On le trouve en Corse, dans le midi de la France, lo Ciers, la Gironde et la forit d'Ortéans. Il habitr, en outre, l'Angleterre, l'llalie, la Sicile, la Grèce, l'ìle de Iilındes, elc. 
D'après un mémoire de M. Ch. Desmoulins sur lo scul Cyclamen qui existe dans la Gironde, il résulterait que cetle espèce, qui parail la même que la nôtre, doit porter le nom de Gyclamen Neapolitatum Ten., et non celui de C. hederaefolinm dis. Kew. Koch. Ce dernier ast adopté par MII. Grenier et Godron, sous te nom de C. repandum Sibt. et Sm. Il fleurit au printemps, et se trouve dans le dépaitement du Gard, près de Nontpellier, à Draguignan et dans les montaunes de la Corse; il a conme synonymes les noms de C. vernum J. Gay, C. ficariifolium Ten.

Quant au Gyclamen Europaeum L., sur lequel il n'existi: pas de doutes, nous l'avons abondamment au pied du Sateve; il se trouve dans le Jura et dauss plusieurs localités de la Suisse, de la France, du Piémont ut de l'Allemagne. Il fleurit de Juillet i Septembre et ses fleurs sont très-odorantes.

L'espece de lioche et du rallon des Usses devrait donc reprendre te nom de Cyclamen Neapolitanum Ten.

Du reste, je ne posside pas d'échantillons de la plante du Salève, el il serait bon de la comparer à celle du canton de Vaud, afin de s'assurer si c'est bien la même espèce.

L.e Cyclamen de Roche flemrit de Septembre it Octolire et ses fleurs sont inodores.

\section{Asperugo procumbens L.}

\section{Syn. : A. vulgaris Dum.}

Ne se trouve que sous les voùtes du Petit-Salève el n'rexiste pas dans notre dura; il est commun le long des rontes, dans les décombres at antour des ehalets du Valais, du canton de Vand et des Grisons. Il parail disséminé dans toute l'Allemagne, la Savoie, le P'ímont, le mirli de la France, l'Espagne, l'Ecosse el Ir nord de l'Angleterre; il habite également la Suède, la Sibérie, l'Asie mineure et le mont Sinaï. 


\section{Pedicularis tuberosa I.}

Syn. : P. gyrollexa $\beta$ Vill., P. ascendens Itopp. el

Stern. Schl. non Gand.

Cette plante des Alpes granitiques a été découverts an Saleve par Mil. Chavin et Rapin, sur les couchess siderulitiques, près de la Croisette, ou elle est rare.

Elle manque dans le Jura el se rencontre sur les hantes Mpes de la Suisse, dans phusienrs localités des cantons de Berne, de Lueerne, de Vaud, dn Valais, des Grisons, du Tressin. Elle a eté sinnalio an HontCruis ot dans les Apes de Fenestrelle. Fo Allomigne,

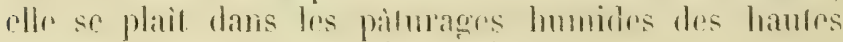
Alpes du 'Tyrol et de la Carinihie.

En France, elle se rencontre sur les monlagnes du Dauphine, de l'Aureronte ot sur les Pyreneses orientales.

La station du Salève serail une des moins élevées de relle esprese; qui preferer los palturages homides des hantes Alpes graniliques.

\section{Plantago cynops $\mathrm{L}$.}

\section{Syn. : P. Genevensis Poir.}

II est surtout ahondant entre Hornex of Honction of sur qualdques points de la plaine; il parut nul dans notro Jula, et n'existe dans le resir de la Suisse que près dre Rolle ef de Nyon ou il ref forl rare. En Allemagne il n'est gnèr signalé qu'aux muirons de Vimme. En revanche, il est abondant an Piémont; on France, on le trouve dans la Côte-d'Or, à Lyon, dans le Dauphiné, dans la région méditerranéo et dans les Pyrénées orientales.

En résumé, cette espèce habite les collines arides ol chandes depuis l'Mlíque septentrionale, l'Espagnt, l'Halie et la Franee méridionale jusqu'en Aulriche of en Asie minemre (Boissier. Voyage en Espagne).

\section{Plantago serpentina Vill. Rent.}

Sin. : P. intergalis Gaud., P. Wulfenii Mort. " Koeh, P. Halleri Schl. exs. 
II courre une plaine angilense entre Archamp ef Sillive, il se retroure prés du Chable el de Saint-Julien. II n'existe pas ailleurs en Suisse.

Allioni le mentionnr en Prímont. En France il at dit signalé dams te Département du Donbs, a Lyon, dans le bauphiné, la l'rovence, le Languedoc, Its Cévennes et les Pyrénées.

lioch indiqur plusiems locialités de Allemagne méridionale.

M. Boissien (Voyatge ren Lipagne) l'at récolté dans la région inférieure de la Sierra Nevada.

D'apres le Prodomm, il habite lon plaines atroilouses rt matilimes de l'Espanne, de l'Ilalie, de la France, de lat Suisse, de l'Allomagne, de la Dahmalie, de la Russie el de l'ile de Sainte-Hélène.

\section{Daphne alpina $\mathrm{L}$.}

\section{Syn. : Thymelea alpina All.}

Cet arbuste odorant se trouve sur les rochers du

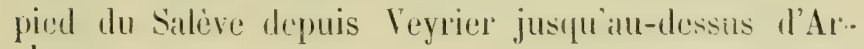
champ.

Il oceupe phisien's points du dura, mais pats dans notre voisinige.

II n'est pas commun en Suisse et il n'est signalí quid dans les eantons de Vaud, du Vatais ef des Grisons.

On le relrouve en Piémont el dans le sud-ouest de lat France; il manque dans les Vosges ed l'Anglesture. En Allenagur, on le rencontre sur les rochers do la phes grandr partice de la chaine des Alpes do Midi.

En résumo, il habite l'lspagne, les Pyrénées, la France méridionate, l'Italie, les Apennins, l'Istrie, la Suisse, l'Aulriche, la Ciroalic, lat Datmatie el la Cairinthie.

\section{Alnus viridis DG.}

Syn .: Belula viridis Chaix in Vill. Gaud.

Cirt arbrissean croit sur les dépòts sidérolitiques du Salève, derriere les l'itons el au-dessus de Cruscil- 
les; il est aux Voirons et au brizon. Je ne crois pas qu'il se trouve sur la chaine Jurassique.

Il est cité anx environs de Baile, dans le Vully, dauss les cantons de Vaud, de Berne, d'Appenzell, d'Uri, du Valais el des Grisons.

En Allemagno, on le trouve daus la Forèt-Noire et dans les terrains granitiques des Alpes, ou il s'élieve très-hant.

En France, il habite les hautes $\Lambda$ pes, surtout colles du Mauphine, d'où il descend dans presque toutes les vallées alpines. II manque dans le Nord et en Anglelerre.

\section{Betula alba L.}

Il se trouve it l'état spontané sur le tevers méridional du Saleve, atl-dessus de Cruseilles. Il est ratre diuns le Jura où il est plutot naturalisé. En Suisse, il oecupe les plaines et les régions subalpines, surtout du còté nord, où il s'éléve jusru'à \& ou 5,000 pieds itudessus de la mer. On le Lrowve c'n Piémont el dans lén terrains sablonneux el siliceux du nort el de l'onest de la France, surtout dans les régions élevées des monlagnes. Il existe en Angleterre et devient toujours plus abondant a mesure qu'on s'avance vers le Nord. Sur le Canigou, il s'bive jusqu'i 6,000 pieds, et datus l'Europe occidentale it s'avance jusqu'au Liap Nord.

Deschampsia flexuosa Girisel.

Syn. : Aira flexuosa L., Avena flexuosa M. et K.

Celle graminée ne se trouve que sur les sables siliceux près des Pitons el audessus de Pommicer. Sille croit aux Voirons, au Mont-Brizon et dans le Jural francais.

En Suisse elle est commune dans les prairics alpines; elle ust indiquée spécialement à Bìle, dims le canton de Vaud el dans celui du Valais.

Fille se plait sur les collines at les montagnes sèches cl stériles de la plus grande partie de l'Allemagne. On 


\section{3}

lit trouve en Piémont, dans loule- France; elle est commune aux environs de Paris, et en Angleterre.

M. Boissier (Voyage en Espagne) l'indique dans les prairies seches de la région alpine de la Sierra Nevada, en lui donnant pour habitation la Suède, l'Amérique septentrionale, le Caucase, l'Espagne, eic. Il fait observer qu'elle n'est alpine que dans l'Europe méridionale.

\section{Lycopodium clavatum L.}

Cielle espèce, qui prélère les terrains siliceux, a cus cependant indiquée à la Dôle par Gaudin, où je l’ai cherchée inutilement. En Suisse el en Allemagne, on la tronve dans les bois et les bruyères humides. Elle existe en Piémont, en Angleterre et en France, sutout sur le grès Vosgien, la Còle-d'Or, l'Auver'gne, le Centre, les Alpes et les Pyrénées.

\section{Asplenium septentrionale Sw.}

Syn. : Acrostichum septentrionale $L$.

Cielte Fougère ne se rencontrean Salève que dans les lissures des blocs erratiques (Esery, La llùre, ete.); on la louve disséminée sur le Jura dans les mèmes conditions. Elle est très-comntme dans lat vallée de Cihamomix el dans le Bas-Valais, le Piémont, l'Allemagne, la Sibéric, l'Angleterte, la France, sur les montagnirs granitiques (Vosges, Mpes, Pyrénées, Cor'se). Elle habite toutes les Mlpes de l'Europe depuis la suede jusqu'i l'Espagn!: elle croil aussi en Asie-Mineure.

Geterach officinarum C. Bauh. Wild.

Syn. : Ilplenium Ceterach 1., Grammitis Ceterach Sw.

Celle espèce est rave pour le Salève; on ne latrouve que sur les rochers at Hornex et sur les murs an pierles sèches de la Mure. Elle est commene en Valais et dans le reste de la Suisse, ainsi qu'en Piémont et en Angleterre. Elle habite l'Europe centrale et méridionale depuis l'Angleterre jusqu'en Grèce, en Espagne et en Afrique (Nord). 
V'igétation comparéc du mont Salève el de la partic Ju Jura qui avoisine lienève.

Lat plupart des plantes qui croissent au Salive se rrtrouvent sur le Jura; ces deux montagnes présentant beancoup d'analogie dans leur formation, leurs rocher's bien exposés, leurs bois de sapins, leur's éboulements calcaire's et leur sol dysgéogène doivent offrir de grandes analogies dans leur végétation. Cóest ce qui a lien, en oflet; cependant il existe certaines diflérences quil est intéressant de signaler.

Salève, outre un certain nombre de plantes qui ne se rencontrent que dans sa dition, possède quelques espèces spéciales vivant sur le sable cristallin et sur les couches siderolitiques qui revetrnt certaines parties de sa surface. Ces plantes, qui se retrouvent se it aux Voirons, soit sur les montagnes primitives, ne se rencontrent pas sur le Jura.

En r vanche, cette dernière chaine, sn raison de la plus grande hauteur des sommets principaux de sa partie méridionale, tels que le Crèt du Miroir, le lieculet, les Colombier's de Giex, la Dòle, le Vuarme ot lo: Marchairu, est riche en plantes alpines qui se retrouvent au Brizon, an Vergy, au Méry el dans la vallée du Reposoir.

Ayant déji douné dans l'introduction la liste des espéces spéciales au Silève, je me bornerai, pour la partie. du Jura comprise entre le Narchairu et le Crề du Miroir, à l'énumération des plantes qui ne croissent pas sur le mont Salève. 


\section{Émumcralion des espèces jurassigues qui ne se rencontrent pas all Salève '.}

Anemone alpina L., A. nareissillora L., Aquilegia illala koch., Aconilum Anthora L. (Doole, Vuame, Ricculn, Vuache), A. paniculatum L. (Faucille, Colombices de (iex), A. Napellus L. (Dóle), Barbarea praecox li. Br. (Credoz), Arabis hrassicaefornis Wallr. (Dòle, licculet), A. Cenisia Reut. (Colombiers de (iex), Erysimmm nehrolencum DC. (Dòle, Colombiers.), Thlaspi Gaudinianum Jord., T. Lersechii Reut., Iberis ceralophylla Reut. (Gingins.), Ethionema saxatile R. Br. (Fort-deI'Rcluse, Vuache), Hutchinsia alpina R. Br.

IIelianthemum grandiflorum DC., II. pulverulentum DC. (Fort-de-l'Écluse.)

Viola palustris I. (Trélasse), V. arenariat DC. (Colonnbiers.), V. canina L y lucorum Reich., V. billora $L_{\text {.., }}$ V. calcarata L., V. sciaphila hoch (Crèt du Miroir, deconverte par M. Rapin le 1/4 Juillet 1867), V. alpestris Jord.

Silene glareosa Jord., S. quadrifida L. (Reculed), Gypsophila repens L., Dianthus rupicola Jord. $\beta$ grandiHera Reut. (Reculet, Dòle), J). carsius Sm. (Recenled), I. Monspessulanus L. (Reculel), Saginal nodosa Fenz!. (Trélasse), Alsine verna Bartl., A. Bauhinorum I. Gay (Reculet, Dóle), Arenaria ciliata L. (Reculet, Crèt de lí (ioulte.)

Linum montanum L. ₹ alpinum lieut., Hypericum fimbriatum L. (Reculer, Dòle), Acer-plalanoides L., A. Nonspessulanum L. (Fort-de-l'Ecluse.)

Geranium Phoum Lam. (Reculet.)

Cytisus alpinus Mill., Oxylropis montana JG. (Rirenlet, Colombiers), Onohryehis montana ICC. (Colombiers), Lathyrus heterophyllus I. (Colombiers), Orobus luteus L. (Reculet, Dôle.)

Rubus Bellardi W. et N., R. rudis W. et N., R. ri-

1 . Te n'indique pas de locilitís spreciales pour les especes yui se trouvent un peu partout sur notre chaîne. 
gridus Merc., H. hispidus Mele., R. veslitus W. el N., li. conspicums Mull., li. Lomentosus borck., Flagaria dumetor'um dord., Polentilla alpestris Ilall. fil. (Reculel, Doile), P. intermedia L. Gaud. (Saint-Georges, Mar'chairu), P. minima Ilall. lil. (lieculet, Crèt des Neiges), Sibbaldia procumbens L. (Reculed, Crèl du Miroir), Alchemilla fissa Schum. Gaud. (Colombiers), Sorbus Channatmespilus Crantz. (Lieculel, Dole) $\beta$ tomentosa Reut. (Dôle.)

Epilobiun rosmanifolimm dacy., E. trigonum Schr. Roel, (Reculet, Dòle), E. palustre L. (Trélasse, (iivine), F. alsincfolim Vill. (Dole, heculel), F. alpinum (L. (Colombier's).

Sedum atratum L., Sempervirum Iuratense Jord., S. Fituconneti Reut. (Montagne de Saint-Jean, pres du Reculet), Rihes petracmu L., Sixiliaga opposililolia L. (lieculet), S. muscoiles Wult. (Colombices, lieculed), S. Hirculus L. (Marais de la Trelasse, Tomblièe des liousses.)

Hryngium alpinun L. (Reculel), liuplevemm longitolium L. (Reculei, Dòle), B. ranunculoiles L. (Recult, Dole, Colombiers), Libanolis monlana L., Ligusticum firulacenm All. (Reculet, Golombiers), Angelicat montana Schl:, Pencedanum Chabraei Gand., P. oreosclimm Moench., Laserpilium latifolium L. $\beta$ atsperum Gaud., Chaerophyllum aureum L.

Lonicera coerulea L. (Marchairu), Galium anisophyllum Vill. Gaud., Valeriana angustifolia 'l'ausch., Cicphalaria alpina Sehrad. (Reculet, Colombiers), Scabiosa lucida Koch.

Putasites allous batertn., I'. niveus Baume. (Colomlicus), Erigeron glabratum Jlopp., Ister alpinus L., Senecio Doronicmon L., Cineratia campestris Ret\% (SaintGeorges, Marchairu), Buphthahmu salicifolium DG., Cinaphalium supinum L. (Preculei), Leontoporlium alpinum Gass. (Dols), Cirsium Erisihales Scop), C. rivulare Link, C. erucaginemm DC., C. subalpinun Gaud., Carrluus mulflorus Ciand. (Saint-Gergnes), C. personata Jieq. (Fancille), Centaturea alpestris llegelsch., 
Serratula Vulpii Fischer-()st., Lilppa pubens Bor., L. Iomentosal Lam (Lavaliay), P'icris crepoides Saut. (Dòle), l'. Villarsii Jord. (vallon d'Ardran), Tragopogon orienrale $\beta$ aureus lieul. (Döle), Taraxacum officinale Migg. var. alpinum Koch. (Reculet), Lactuea perennis $\mathrm{L}$. (Fort-de-l'Ecluse, Vuache), Mlulgedium alpinum Less. DC. (Dòle), Crepis snccisacfolia Tausch. (Dòle, lieculet), C. blattarioides Tausch. (Dóle, Reculet), Soyera monlana Monn. (Döle), Ilieracium aurantiacum L. (Reculel). II. tlexuosum W. et K. $\beta$ calvum Gir. el G. (Dòle), II. elongatum Willd. (Dòle, Reculet, Colombiers), II. dentatum Hopp. (vallon d'Ardran), Il. Vugesiacum Moug. (Dòle, Reculet), II. porrechun Fries. (Reculet, montagne de Saint-Jean), H. clatum Fries $\beta$ Laggeri lieut. (Faucille.)

Phyteuma orbicularis L., Campanula thyrsoidea L., C. Latifolia L., C. rotundifolia L. vall'. confertifolia L., (chalet de Thoiry.)

Vatcinium ulighosum L., Oxycoccos vulgaris Dun., Andromeda polifolia L. (Trúlasse), Aretostaphyos alpina Spring. (montagne d'Allemogne), lihododendron ferrugineum L., Pyrola uniflora L. (Coinsins.)

Androsace villosa L. (Dôle, Vuarne et non pas le Vuarhe), Soldanella alpina L. (Reculet, Dôle.)

Swertia perennis L. (Trélasse), Gentiana pneunonanthe L. (Pied), G. Clusii Per. el Song. (Reculed, Dôle).

Cerinthe alpina Kit. Koch (Siml-Georges), Myosotis alpestris Schulz. (Dôle, Reculet.)

Orobanche epithymum (DC. Doile, Reculet).

Serophularia Juratensis Sch., Linaria petraeat lord. (Reculet), Digitalis media Roth. (Saint-Cergues, Ciretde-la-Goutte), Veronica aphylla L., V. saxatalis L. (Crèt- (du-Mliroir), V. nummulariodes Lec. el Lam.), Tozzia alpina L. (Dòle, Fancille), Pedicularis foliosa L. (vallon ('Ardran), Bartsia alpina L., Odontites lutea Reich. (Thoiry), Euphrasia nitidula Reul. (Dòle), E. mininat Jacq. (Dôle.)

Salvia verlicillala L. (all-dessus de Farges, décourerte 
le 14. duillet 1867, en compagnie de MM. Reuter, Rapin, Ayas et Mellord), siderilis hyssopifolial L. (Thoiry, Colombiers, Dòle.)

Pinguicula grandillora L. avee la valr. $\beta$ prallidia Giaul. (Faucille, Reculet.)

Plantago montana Lam., Rumex sculatus L., Polygonum bislorla L., P. viviparum L.

Daphne Cneorum L. (Marchairu.)

Enpetrum nigrum L. (monlagne d'Allemogne, Trílasse), Euphorbia rertucosa L. $\beta$ montima (iand.

Salix pentandra L., S. ambigua Ehrh. (Trélasse), S. Irusa L. (Döle, Reculet, Colounbiers.) S. reticnlatit L. (Reculel), Betula pubescens Ehrh. Koch. (Trólasse.)

Juniperus Thana Willd. (Doble, Recules), Latlix Europaca DG. (Faucille.)

Seheuchzeria palustris L. (Rousses), Polamogeton rulescens Schrad. (Etang de la Givrine-)

Nitritella suaveolens Koch (Döle), Epipogium Gimrlini Rich. Koch (trouvé par M. Bornett ind Juilled $18666^{\circ}$ dans lus bois de hêtres, all-dessus de Gingins, prè des Longes), Epipaclis microphylla Sw. (mene lor:alité), Listera cordata Rich. (Doile, Faucille, Bois audessous du Crêt-de-la-Goulle.)

Narcissus radiiflorus Salish. (Pàturages de la Dòl:s), Streptopus amplexifolius DG. Dote, Fancille), Ruscus aculeatus L. (Fort-de-l'Echise, Vuache), Mllium victorialis L. (Doble, lireulet), A. lallax Don. Koch. (Reculct, Colombiers), A. Sibiricum L. (Dole), Paradisia Liliastrum Bert. (Reculet, Dòle), Veratrum album L. et var. $\beta$ Lobelianum Koch.

Luzula multiflora Lej., $\beta$ congesta Gr. cl G. el $\gamma$ nigricans Gr. el G., L. spicati DC. (Reculet, Dòle) Scirpus coespitosus L. (Rousses), Eriophorum alpinum L. (Trélasse, Tourbière de la Pile), Carex pancillora Light. (mème localité) C. teretiuscula (iood (Givrine el Trélasse) C. canescens L. Koch. (Trélasse), C. chlorocurpa Wimm. (Trélasse, Tourbière de la Pile), 
C. Limnsa L. (Trúlasser.) C. ferruginea Seop. Gor. (Dòle, Reculet), C. filiformis L. (Trélasse,)

Phleum alpinum L. $\beta$ foliosum Reul. (Nontagne de Saint-Jean) y commutalmm Reul., P. Michelii MII. (Dile), Igrostis vulgaris With. $\beta$ aristata Reut. (sur Thoiry), A. Schleicheri, Jord. Verl. (Reculet, Colonbier's, Faucille), Deschampsia corspilosa P. B. $\beta$ alpina Gand. Trisrum Gaveseens P. B. $\beta$ varingata Ciand., Poa sudetica Ilaenk (Saint-Cergues), P. hybrida Gand. (Doble, Fancille, Rerulci), P. ferlilis IIosi. (Fan-

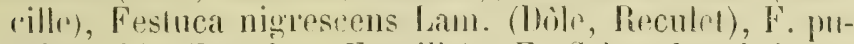
mila Vill. (Reculet, Fancille), F. Schenchzeri Gand. (Vallon d'Ardran, Colombiers).

Phegopteris alpestris. Mell. Bernouil. (Dole, Fancille, Coloubiers), Polystichum Oreopteris DC. (Lavitlay), Cystoptris regia Prest. Koch avee la var. $\beta$ alpina Koch (Döle, lieculet, Crit-des-Neiges, Faucille), Adianthum Capillus-Yeneris L. (Credoz), Lycopodium annotinum L. (Dồle).

Obs. Les Arabis alpina L., Cardamine pratensis L., Dryas octopetalas L., Saxifraga aizö̈des, L. Chrysosphrnimm altersifolimm L., Taraxacum offecinale L. Festuca oxina L. (qui habitent Salève of le Jura). Saxifraga oppositifolia L., S. Itirculus L., Empetrum nigrum L., Polygonum viviparum L. ef Salix reticulata L. (qui habitrnt le Jura te ne se tronvent pas a Saleve) font partie des 93 vigntanx phanerogames qui conslituent la Flore du Spizberg (Ch. Martins). 


\section{Note sur les plantes hybrides observées aux penvirons de Genève.}

Bien que l'on puisse admeltre en théorie et que la pratique prouve qu'il existe des hybrides de plantes annmelles, je n'en connais ceprendiut point dans les limiles de la Flore de Geneve; loutes les formes que nous possédon̂s se rapportent à des végétaux bisamnuels on vivaces.

Il existe dans In midi de la France une forme hybride do plantes annuelles, l'Egylops triticoides Req. (E. ovala var. trilicoides Jord. E. vulgari ovatum Godr. et Gren.), qui a donné lieu à une polénique des plus intéressantes entre MMi. Iordan et Godron. Celte forme serait le résultal du croisement de l'Egylops ovata L. et du Tritieum vulgare Vill. d'engage les botanistes qui s'intéressent anx questions d'hybridité, it lire les hrochures de M. Iordan (Alrmoire sur l'Egilops trilicoides, 1856. - Nonveau Mémoire, 1857) el de M. Godron (Rapport sur le Traité de Lecoq, 1840.- He l'Agylops triticoides el de ses différentes formes, Annales des Sciences naturelles, 4me Séric, T. V).

On a donné souvent te nom spécifique d'hybride i des plantes qui sont de bonnes et de légitimes espèces so reproduisint par graines et présentant des caractères conslants. Cí nom leur a élé imposí peut-être parce qu'elles présentent des caractères qui les rapprochent d'rspeces voisines, mais dont elles se distinguent spéritiquement. Je citerai comme exemple : Trifolium hybridum L., Mehrumilla hybrida Willd., Sorbus hybrida I., Lamium hybridum Vill. DC., Chenopodimm hylnidum L.', Poa hybrida Gaud., etc.

Lus véritables hybrides sont des végétaux qui liennent te milien entre tes parents qui laur ont domé naissance; ils ne se rencontrent que lit où croissent les plantes desquelles ils dérivent; ils sont tomjour's

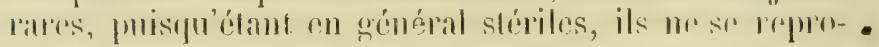


duisent pas enx-mòmes, at moins qu'ils n'aient des souches trateantes et des stolons. (Ouand il leur arrive d'avoir des semences fertiles, le produit de ces graines retourne à l'un des parents, ordinairement au type maternel.

P'uisque ees fommes existent dams la mature ef yu'on no pent pas les snppriner, il faut bien les dérirr, mais je ne les considere que comme des variótes acridentelles et non comme de vérilables espèces.

Onoi que la nomenclature arloptée généralement pour leur domer des noms soil passablement batbare, cosl. encore cello qui convient le mirmx ponr éviter des confusions, en faisant connaitre en même temps al la nomrelle lorme el les parents qui lui onl dommi le jour.

Voigi maintenant la liste des hybrides que j’ai ju observer dans nos environs.

Arabis hybrida Reut. (A. murali-stricla).

Modicago media Pers. Bor. (M. filcato-sativa Rurich).

Iiubus idaco-coesius E. Merc., li. patens E. Mcre. (R. dumeloro-rusticanus), R. Psudo-coesius E. Mrere. (li. coesio-dumetorum), Rosa alpino-pimpinollifolia lieul., R. pimpinellifolio-alpina liapin., R. Chavini Rap.. (R. montano-canina), R. gallico-canina lieul., R. liybrida Schl. (R, arvensi-gallica), R. gallico-mmbellata liap), Aronia Aria-chamacmespilus Reich. (Sorhus chamaemespilus Crantz. $\beta$ tomentosa Reut.)'.

Epilobium parilloro-palustre Ilanpp., Saxifraga nufato-aizoides Reut., Galium vero-molluego Wallr.

Inula somi-amplexicaulis Reut. (I Salicino - Vaillantii), Cirsium palustri-acatule Rap., C. hy̧bridum Koch (C. palusuri-oleracemu Nay.), C. erucagincum Gaud. (C. rivulari-oleracem Nage.), C. Lachenalii Gnel. (C.

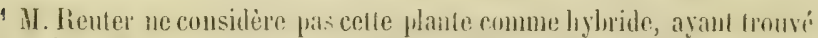
dr's graines mures arec des embryons parfaitement conformés; cepru-

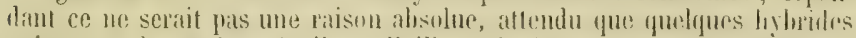

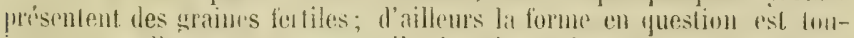
jours rare, elle ne se tronve yue lit wi croissent les pirents, ef ello lim complétement le milieu entreux pour l'aspect el les caractères. 
oleraceo-araule Ilamp. Næeg.), C. palus(ri-acaule Hamp., Hieracium pilosello-pracallum Sch.

Primula officinali-elatior Muret., P. variabilis Gonp. (P. officinali-acaulis Mlure.), P. acanli-clatior Murel., P. acauli-suaveolens.

Guntiana Thomasii Gill. (G. purpureo-lutea Griseb) ), F. Charpentieri Thom. (G. punclato-lutea Griseb.).

Verbascum thapsiformi-floccosum koch, V. Jyehnitidi-flocestum Korh, Y. nigro-flocosum lioch, Y. Iychnilidi-blallatic Koch, V. llapsiformi-blattaria Gr. nt G., V. Hhapso-lychmilis II. el K., V. nigro-ihapsus Fries, V. nigro-focesum koch, V. nigro-Jychnitis Schirel, V. Hocesoso-lhapsiforme Wirtg., T. nigro-lhapsiforme liap.

Digilalis media Roth. (D. Luteo-grandiflora.)

Mentha sylvestri-rotundifolia Wirtg., II. nepetoides Lej. (II. agmatico-sylvestris Mey.), M. sativa L. (MI. arpatico-arvensis Wirlg. ou hirsuto-arvensis Rap.), M. arvensi-pulegium Reul., Stachys ambigua Sm. (S. sylvatico-palustris.)

Salix Wimmeri Kern. (S. incano-diphnoides Wim. Reur.), S. Pontederant Willd. Gand. (S. cinereo-nigrricans Reut. on purpureo-cinerea Rap. on purpureodaphnoides Chavin), S. Seringeana Gaud. (S. incanocaprae Chavin.)

Orehis sinnio-purpurea Weid., 0. purpureo-militaris Gr. el G., 0. morio-laxiflora Reut. Risch., Nigritelli nigro-conopsea Reich., Nigritella suaveolens liorh (N. nigre-odoralissima). Agrostis canino-rulgaris Nere. 


\section{Additions et corrections.}

Page 12. Uryonicn et Níocomien, lisez: Néocomien at Urgonien.

P. 13. Après Lycopodium clanatum L., ajoutez: Pedicularis tuberosa $\mathrm{L}$.

P. 15. Asplenium Septentrionale L., lisez: Asplenium Septentrionale Sw.

P. 15. Ceterach officinamun CB., lisez: Ceterach officinurum C. Baul.

P. 15. Cynoglossum montanum L., lisez: Cynoglossum montanum Lam.

P. 16. Après Evonymus latifolius 1., ajoutez: Dipisacus laciniatus $\mathrm{l}$.

P. 19. à Salève, lisez: au Salève.

P. 21. avellana, lisez: Avellana.

P. 22. Chamcbuxus, lisez: Chamaebuxus.

P. 24. Petusites rulguris Hoench, lisez: Petrsites officinalis Moench.

P. 24. Fraximus elatior L., lisez: Fraximus excclsior L.

P. 25. Arum vulgare L., lisez: Arum maculatum $L$.

P. 26. petraa, lisez: petraea. Europœus, lisez: Europaeus.

P. 28. Arabis saxatilis L., lisez: Arabis saxutilis All. 
1. 29. Europoeus, lisez: Europaeus.

P. 29. Malus communis L., lisez: Malus communis Poir.

P. 30. Spirce aruncus L., lisez: Spiraed Aruncus L.

P. 30. Orchis Simia L., lise\%: Orchis Simit Lam.

P. 30. Helianthenum, lisez: Helianthemum.

P. 32. Myosotis sylvatica L., lisez: Myosolis sylvatica Ehrh.

P. :32. Actera, lisez: Actuen.

P. 4. grandiflora Baib., lisez: grandifloren Bab.

1'. 40̃. luciniatus, lisez: laciniatus.

P. 促. B Salaevense, lisez: $\beta$ Salaerense.

[?. 65. A. arcutu, lisez: A. Arcuatu.

I. 71. L. alpestris Ilopp., lisez : L. sylrestris Hopp.

I. 85. R. sphaericu Grem, lisez: R. sphatericn Gren.

I. 85. $\gamma$ hiserata liap., lisez : $y$ biserrala Rap.

I. 94. L. sanguin, lisez . C. sanguin.

1. 95. Après G. boreale L., ajoutez: G. elongatum Presi. Marais de Troinex. Mai, Juin.

I. 45. var. $\beta$ vaillentii, lisez: var. $\beta$ Vaillantii.

P. !8. Après L. corymbosum Gr. et G., ajoutez: L. montaum Koch. (C. leucanthemum II, montanum Gaud.) L. des montagnes. Rocailles du pied et de la région moyenne. Juin, Juillet.

P. H1. C. montanum L., lisez : C. montanum Lam. P. 199. Après Cephalanthera pallens Rich. Koch., ajoutez comme synonyme: (C. grandiflora Bah. ).

P. 1.5. Linum montanum L. $\beta$ alpinum Reut., lisez:

Linum alpinum I. $\beta$ montanum Reut.

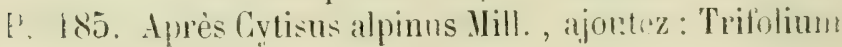
coespitosum Reyn. 


\section{5}

P. 189. Après Cystopteris regia Presl, ajoutez: Cystopteris montana Link. (Grêt des neiges, Faucille).

P. 192. S. incano-caprae Chavin., lisez: S. incanocapraea Chavin.

II. V. Table des espèces. Après Arclostaphylos officinalis W. et G., ajoutez: A. alpina Spreng.

he mapereois - mais un peu tard - qu'il n'est pas facile de mener a bien un onvrage si peu compliqué qu'il paraisse. Ainsi jaurais dù indiquer un plus grand nombre d'especes campagnardes qui aiment ì s'élerer sur la montagne. Mème il eùt été plus court de donner la liste des plantes sédentaires qui alfectionnent certaines localitis de la plaine, et qu'on ne trouve pas ailleurs. En effel, sur lns 1850 végéfaux phaneragames du Catalogue de Reuter, il n'y en a guère plus de 200 que je ne pense pas avoir jamais vus sur le Salève. Il ne me reste qu'à réclamer l'indulgence du lecteur pour les nombreuses imperfections de ce travail. 



\section{TABLE GÉNÉRALE}

Pages.

Dédicace . . . . . . . . . 3

Prélude. . . . . . . . . . . . 5

Introduction . . . . . . . . . 11

Jerborisations. . . . . . . . . 19

Calalogue . . . . . . . . . . . . 59

Notes sur les plantes les plus intéressantes du Salève . . . . . . . . 149

Végétation comparée du Salève et de la partie du Jura qui avoisine Genève. . 18/ Énumération des espèces Jurassiques qui ne se rencontrent pas au Salève . . 185

Note sur les plantes hybrides observées aux environs de Genève . . . . . 190 Additions et corrections. . . . . . 199 Table alphabétique des espèces. 



\section{TABLE DES ESPEECES}

Abies excélsa DC. 52, 125.

pectinata DC. 52, 125.

Acer campestre L. 29, 75 .

Monspessulanum L.' 185.

opulifolium Vill. 26, 29, 55, 74.

- platanoides L. 185.

pseudo.Platanus L. 29, 74.

Achillea Millefolium L. 104 .

$\Lambda$ conitum Anthora L. 64, 185.

lyeoctonum L. 44, 65.

Napellus L. 185.

paniculatum Lam. 185.

Acrostichum septentrionale L. 145 .

Actaea spicata L. 52, 64.

Adenostyles albifrous Reich. 48, 97.

alpina Bl. et Fing. 55, 97.

Adianthum Capillus Veneris L. 189.

Adoxa moschatellina L. 25, 94.

Æ̇gopodium podagraria L. 58. 95.

સthionema saxatile R. Br. 185.

Atthusa cynapium L. 93.

Agropyrum caninum R, et S. 141.

Agrostemma Gythago L. 34, 71 . 
$\Lambda$ grostis alba L. Schr. $\gamma$ gigantea Rent. $52,157$.

gigantea Gaud. 157.

interrupta L, 57, 138.

Schleicheri Jord. 189.

vulgaris With. 158, 142.

var. pumila Gand. Reut 51, 138.

A ira cristata L. 159.

flexuosa L. 158.

Ajuga Genevensis L. 117.

reptans L. 117.

Alchemilla alpina L. 40, 48, 86 .

fissa Schum. Gaud. 186.

hybrida Willd. 48, 86.

pubescens Koch. 86.

truncata Tauseh. 86.

vulgaris L. 40, 48, 86 .

var. $\gamma$ Gand. 86.

Alisma Plantago L. 126.

Alliaria officinalis Andrz. $54,68$.

Allium carinatum L. ๖ั1, 15 .

fallax Don. 188.

sphaerocephalum L. 57, 155.

ursinum L. 24, 155.

Sibiricum L. 188.

Victorialis L. 188.

Aluns glutinosa Gaertn. 21, 125.

incana DC. $21,124$.

viridis DC. $15,55,56,41,124$.

Alsine Banhinorum J. Gay. 185.

fasciculata M. et K. $\mathbf{3 7}, \mathbf{7 2}$.

hybrida Jord. 5ร, 72.

laxa Jord. 5 בั, 72. 
Alsine media L. 75.

tenuifolia Crantz. $\mathbf{3 5}, 75$.

$\beta$ viscidula Gaud. 51, 55, 72.

verna $B$ artl. 185.

Althaea hirsuta L. 57, 73.

Alyssum calycinum L. 34, 68.

Anacamptis pyramidalis Rich., Koch. 128.

Amelanchier vulgaris Moench. 29, 87.

Anagallis coerulea Schr. 34, 109

phoenicea Lam. 54, 109.

Anchusa Italica Retz. 38, 110.

Andromeda polifolia L. 187.

Andropogon Ischaemum L. 57, 157.

Androsace villosa L. 187.

Anemone alpina L. 185.

Hepatica L. 62.

nareissiflora L. 185.

nemorosa L. 25, 61.

ranunculoides L, 27, 62 .

Anethum Foeniculum L. 91.

Angelica montana Schl. 186.

sylvestris L. 48, 93.

Antennaria dioica Gaertn. 99.

Anthemis Cotula L. รั5, 99.

Anthericum Liliago L. 44, 135.

ramosum L. 44, 135.

Antirrhinum alpinum L. 112.

Cymbalaria L. 112.

Elatine L. 112.

minus L. 112.

spurium L. 112.

Anthoxanthum odoratum L. 50, 156.

$\beta$ villosum Reich. 50, 156. 
Anthriscus abortivus Jord. 47, 95.

alpestris W. et G. 95 .

cerefolium L. 95.

cieutaria Dub. 95.

sylvestris Hoffm. 93.

sylvestris var. alpestris Rap. 95.

vulgaris Pers. 54, 95.

Anthyllis montana L. $50,78$.

vulneraria $\mathrm{L} .58,79$.

Apargia hastilis Willd. Gand. 101.

hispida Gaud. 101.

Apera interrupta P. B. 158.

Aquilegia atrata Koch. 185.

vulgaris L. 51, 65.

Arabis alpestris Schl. Reich. 32, 49, 65.

alpiṇa L. 31, 52, 65.

arcuata Schuttl. $\beta$ hirsuta God. 65.

auriculata Lam. 51,65 .

brassicaeformis Wallr. 185,

Cenisia Reut. $185^{\circ}$.

ciliata $\beta$ hirsuta Koch. 65.

conferta Willd. 65 .

hirsuta Scop. 51, 65.

hirsuta I sessilifolia Gaud. 63.

hybrida Reut. 15, 51, 66, 153 .

incana Roth. 51, 65.

muralis Bert. 51, 66.

sagittata Bertol. DC. $\mathbf{3 6}, \mathbf{6 5}$.

saxatilis All. 28, 63.

serpyllifolia Vill. 42, 66 .

stricta Huds. 51, 66.

Thaliana L. 68. 
Arabis 'Turrita L. $51,44,66$.

Arbutus Uva-Ursi L. 107.

Arctostaphylos officinalis W. et G. $2 \ddot{3}, \mathbf{5 0}, 107$.

Arenaria ciliata L. 185 .

fasciculata Jacq. 72.

grandiflora I. 28, 34, 72, 157.

leptoclados Guss. 55, 38, $7 \mathbf{5}$.

serpyllifolia L. $\mathbf{5 8 , 7 5 . ~}$

sphaerocarpa Ten. 55, 58, 75.

tenuifolia L. $\mathbf{7 2}, \mathbf{7 5}$.

var. hybrida Vill. 72.

trinervia L. 72.

viscidula Thuil. 72.

Aruica montana L. 13, 42, 98. 171.

Arrhenaterum elatius M. et K. 55, 138

Arum maculatum L. 25, 126.

Arundo Epigeios L. 157.

Asarum Europaeum L. 20, 121.

Asclepias Vincetoxicum L. 109.

Asperugo procumbens L. 16, 57, 111, 179.

Asperula arvensis L. $\mathbf{5 4 , 9 3}$

cynanchica L. 95.

galioides DC. 95 .

odorata L. 32,95 .

Aspidium aculeatum Döll. 57, 144.

dilatatum Godr. 144.

Filix-mas Sw. 144.

fragile Sw. 144.

lonchitis Sw. $58,145$.

rigidum $\mathrm{Sw}$. 144 .

spinulosum Döll. 144.

'Thelipteris Sw. 144 .

Asplenium Adianthum-nigrum L. 5้7, 145. 
Asplenium Filix-foemina Bernh. 57, 144.

Halleri DC. 57, 144.

Ruta-muraria L. 57, 144.

Scolopendrium L. 14.5.

septentrionale Hoffm. 15, 57, 145.

T'richomanes L. 57, 144.

viride Huds. $57,144$.

Aster alpinus L. 186.

amellus L. 55, 97.

Astragalus Cicer L. 78.

Astrantia major L. 40, 48, 91 .

Athamantha Cretensis L. 53, 92.

Athyrium Filix-foemina Roth. 144.

Atragena alpina L. 15, 52, 61, 149.

Atriplex hortensis L. $50,120$.

patula L. $50,120$.

Atropa Belladona L. 47, 111.

Avena elatior L. Gaud. 158.

fatua L. $38,158$.

flavescens L. 159.

flexuosa M. et K. 158.

orientalis Schr. $38,142$.

pratensis L. 41, 45, 158.

pubescens L. $\beta$ alpina Gaud, 189.

sativa L. 58, 142.

Barbarea arcuata Reich. $16,46,65,155$.

Barkhausia foetida DC. 102.

Bartsia alpina L. 187 .

Bellidiastrum Michelii Cass. 50, 97.

Bellis perennis L. 104.

Berberis vulgaris L. 29, 64.

Beta vulgaris L. 50, 120. 
Betonica officinalis L. 51,116 .

Betula alba L. 15, 25, 56, 124 .

alnus $\beta$ incana $L .124$.

; glutinosa L. 125.

pubescens Ehrh. 188.

viridis L. Gaud. 124.

Blechnum Spicant Roth. 57, 145.

Blitum virgatum L. 5 ร, 119.

Botrychium Lunaria Sw. 18, 145.

Brassica oleracea L. 55, 68.

orientalis L. 67.

Napus L. 5.j, 68.

Rapa L. 35, 68.

Briza media L. 139.

Bromus asper L. 48, 141.

erectus Huds. $\mathbf{3} 5,141$.

giganteus L. 141.

sterilis L. 34, 141.

Brunella alba Poll. 116.

grandiflora-Moench. 55, 117.

laciniata Lam. $55,116$.

vulgaris $\beta$ laciniata $L .117$.

Bunias erucago L. 42, 68 .

Bunium Bulbocastanum L. 91.

Buphthalmum salicifolium L. 186.

Buplevrum longifolium L. 186.

ranunculoides L. 186.

Buxus sempervirens L. 22, 24; 121.

Cacalia albifrons L. 97.

alpina L. Gaud. 97.

petasites Lam. 97.

Calamagrostis argentea DC. 138. 
Cala magrostis epigeios Roth. 157.

littorea DC. 157.

montana Host. 48, 157.

Calamintha alpina Lam. $49,55,115$.

ascendens Jord. 5ั, 11 s.

menthaefolia Host. 115.

nepeta Gaud. 115 ,

nepetoides' Jord. $55,115$.

officinalis Benth. 115.

Calluna vulgaris Sal. 56, 107.

Caltha palustris L. 55, 65.

Camelina sativa Crantz. 34, 67.

Campanula aggregata Noc. et B. 85, 103.

cervicaria L. $51,105$.

glomerata L. 40, 105 .

latifolia L. 187.

patula L. 42, 47, 106.

persicifolia L. 45, 106 .

pusilla Gand. 105.

- rapunculoides L. 55, 105.

rapunculus L. $38, \mathbf{1 0 5}$.

rhomboidalis L. 41, 49, 105 .

subramulosa Jord. 53, 105.

thyrsoidea L. 187.

Trachelium L. 48, 105.

$\beta$ dasycarpa Gr. 48, 105.

urticaefolia Schm. Gaud. 105.

Cannabis sativa L. 122.

Capsella Bursa-Pastoris Vent. 49, 68.

Cardamine amara L. 28, 55, 66.

hirsuta L. 35, 68 .

Impatiens L. $55,66$.

Matthioli Moret. $56,66$. 
Cardamine pratensis L. $26,53,56,66$.

$$
\text { y Hayneana Reich. } 66 .
$$

sylvatica Link. 42, 66.

Carduus acaulis L. 99.

arvensis Willd. 99.

defloratus L. $55,100$.

eriophorus L. 99.

multiflorus Gaud. 186.

personata Jacq. 186.

Carex alba Scop. 50, 155.

alpestris All. 155.

ampullacea Good. 27, 156.

brachystachys Schr. 156.

canescens L. Koch. 188.

chlorocarpa Wimm. 188

clandestina Good. 155.

coespitosa Gaud. 155.

Davalliana Sm. 28, 155.

digitata L. $30,135$.

dioica L. 28,135

erecta DC. 136 .

ferruginea Scop. God. 189

filifurmis L. 189.

flava L. 28, 136.

Carex fulva DC. 156.

fulva Good. 156.

glanca Scop. $25, \mathbf{1 5 5}$.

Goodenowii Gay. 155.

gynobasis Vill. 50, 1550.

Halleriana All. Gr. et G. 155.

Hornschuchiana Hopp. 28, 136.

$\beta$ xanthocarpa God. 28, 156.

humilis Leyss. $50,40,155$. 
Cerex lepidocarpa Tausch. 28, 156. leporina L. $50, \mathbf{1 5 5}$.

limosa L. 189.

maxima Scop. $51,136$.

montana L. 25, 153 .

nitida Host. 27, 135.

ornithopoda Villd. $50,155$.

ovalis Good. 135.

pallescens L. $50,156$.

paniculata Is. 27, 155.

patula Scop. 156.

pauciflora Ligth. 188.

pedata DC. 135.

pendula Huds. 156.

praecox Jacq. 25, 155.

remota L. 45, 155.

sempervirens Vill. 55, 41, 42, 156.

stellulata Good. 35, 41, 135.

sylvatica Huds. 156.

tenuis Host. 55, $42, \mathbf{1 5 6}$.

teretiuscula Good. 188.

vulgaris Fries. 55, 41, 155.

Carlina acaulis L. 55, 100.

vulgaris L. $56,100$.

Carpinus Betulus L. 24, 30, 125.

Carum bulbocastanum Koch. 51, 91.

Carvi L. 40, 48, 91.

Castanea vulgaris Lam. 122.

Caucalis daucoides L. 58, 92.

grandiflora L. 92.

Centaurea cyanus L. 38, 100.

Jacea L. 52 - 100,

montana L. 52, 100. 
Centaurea nudicaulis L. 100.

scabiosa L. $\beta$ petrophila Rent. $51,100$.

Centunculus minimus L. $544,109$.

Cephalanthera ensifolia Rich. 44, 129.

grandiflora Bab. 44.

pallens Rich. Koch. 129.

rubra Rich. 44, 150.

Cephalaria alpina Schr. 186.

pilosa Gr. et G. $55,96$.

Cerastium arvense L. $\beta$ strictum Rent. 51, 75.

brachypetaltm Desp. 55, 75,

glomeratum Thuill. 75 .

glutinosum Fries. 35, 75.

obscurum Koch. 75.

semidecandrum L. $55,75$.

strictum L. 75.

triviale Link. Koch. 75.

viscosum L. 5̋, 75.

vulgatum $\mathrm{L} .53 \mathrm{~s}, 75$.

$\beta$ aipinum Gr. et G. 40, 72.

Cerasus avium DC. 29, 80.

Mahaleb DC. 29, 80.

Padus DC. 46, 80, 159.

Cervaria glanca Gaud. 92.

Ceterach officinarum C. B. 15, :37, 145, 183.

Chaerophyllum aureum L. 186.

Cicutaria Vill. 42, 47, 92.

hirsutum Reut. 92.

Chara capitata Nees. 146.

hispida L. ร1, 146.

synearpa Thuill. 146. 
Chelidonium majus L. $\mathbf{3 4}, 64$.

Chenopodium album L. 50, 119.

Bonus-Henricus L。 50, 119.

glaucum L. 50, 119.

hybridum L. $50,119$.

murale L. 50, 119.

polyspermum L. 50, 119.

vulvaria $\mathrm{L}$. 50, 119.

Chironia Centaurium Sm. DC. 110.

Chlora perfoliata L. $\beta$ pusilla Gaud. 109.

Chlora serutina Reich. 54, 109.

Chondrilla muralis Lam. 101.

Chrysanthemum corymbosum L. Gand. 98

lencanthemum L. 104.

I triviale Gand. 104.

II montanum Gaud.

montauum Koch.

Chrysosplenium alternifolium L. 53, 91.

Cineraria campestris Retz. 186.

Cireaea Lutetiana L。 $\mathbf{5 1}, 89$.

Cirsium acaule L. 48, 99.

arvense Lam. 58, 99.

eriophorum Scop. รั5, 99.

Erisithales Scop. 186.

erucagineum DC. Gaud. 186, 191.

hybridum Koch. 191.

Lachenalii Gmel. 191.

oleraceo-acaule Hamp. Naeg. 192.

palustri-acaule Rap. 191.

palustri-oleraceum Naeg. 191

rivulare, Link. 186. 
Cirsium rivulari-oleraceum Naeg. 191.

Cladium Mariscus R. Br. 45, 154

Clematis alpina Mill. DC. 149.

vitalba $\mathrm{L}$. 50,61 .

Clinopodium vulgare L. 51, 115.

Cochlearia saxatilis Lam. 67.

Colchicum autumnale L. 57, 155.

Comarum palustre L. 47, 82.

Couringia orientalis Andrz. 38, 67.

Convallaria bifolia L. 151.

maialis L. 50,151 .

polygonatum L. $30,131$.

verticillata $\mathrm{L}$. $50,151$.

Convolvulus arvensis L. 58 .

Corallorhiza Halleri Rich. 150.

innata R. Br. 48, 130.

Cornus sanguinea L. 29, 94.

Coronilla emerus L. 29, 78.

minima DC. 78.

vaginalis Lam. 30,78 .

Corydalis bulbosa DC. 22, 64 .

cava Wahl. Gaud. 64.

solida Sm. Gaud. 64.

tuberosa DC. 20,64 .

Corylus Avellana L. 21, 125.

Cotoneaster tomentosa Lindl. 29, 87.

vulgaris Lindl. 29, 87.

Crategus Amelanchier Gand. 87.

Aria L. 88.

a Scaudica L. 88.

monogyna Jacq. 86

oxyacantha L. $29,86$.

oxyaeantha Koch. Gaud. God. Rap. 86. 
Crategus oxyacanthoides Thuil. 29, 86.

torminalis L. 88.

Crepis aurea Cass. 48, 102.

biennis L. 48, 102.

blattarioides Tausch. 187.

foetida L. 54, 102.

paludosa Moench. 102.

succisaefolia Tausch. 187.

Crocus vernus All. 55, 151.

Cyclamen Europaeum L. $55,108$.

hederaefolium Ait. 16, $56,108,178$.

Cynoglossum montanum Lam. 15, 32, $111,177$.

officinale I. 45, 111.

Cynosorus cristatus L. 140.

Cypripedinm Calceolus L. 52, 150.

Cystopteris fragilis Bernh. 57, 1/4.

montana Link. 195.

regia Presl. Koch. 180.

$\beta$ alpina Koch. 180.

Cytisus alpinus Mill. $18 \%$.

Laburnum L. $29,77$.

Dactylis glomerata L. 140.

Danthonia decumbens L. 140.

Dapline alpina L. 50, 45, 121, 181 .

Cneorum L. 121, 188.

Lalleola L. 20, 121.

Mezereum L. 20, 120.

Daucus carota L. $气 0,95$.

Delphinium Consolida L. 55, 65.

Dentaria digîtata Lam. 50, 52, 66.

pinnata Lam. 50, 52, 66.

Deschampsia flexuosa Griseb. 15, 49, 158, 182. 
Dianthus coesins Sm. 185.

Carthusianorum L。 40, 71 .

Monspessulanus L. 18 s.

prolifer L. 44, 71 .

rupicola Jord. 44, 71.

$\beta$ grandiflora Reut. 183 .

sylvestris Gaud. 71.

Digitalis grandiflora Lam. 45, 55, 112.

lutea L. $45,112$.

media Roth. 187.

parviflora Lam. 112.

Digitaria filiformis Koch. 137.

Dipsacus laciniatus L. 47, 96.

pilosus 4. 96 :

Doronicum Pardalianches L. 55, 98, 170.

Bellidiastrum L. 97.

Draba aizoïdes L. 20, 35, 67.

verna I. 68 .

Diosera anglica Huds. 69.

longifolia L. 51, 69.

obovata M. et K. 51,69 .

Diyas ociopetala L. $41,49,80$.

Echinospermum Lappula Lehm. 57, 111.

Elymus Enropaeus L. 48, 141.

Empetrum nigrum L. 188

Epilobinm alpinum L. 186.

alsinaefolium Vill. 186.

angustifolium L. Gaud. 88.

collinum Gmel. 47, 88.

Lamyi Schultz. 51, 88.

montanum L, 45.88.

$\beta$ collinum Koch. 38 . 
Lipilobium palustre L. 186.

parvifloro-palustre Hamp. 191.

rosmarinifolium Jacq 186.

spicatum Lam. $51,88$.

tetragonum L. $\beta$ Lamyi Rap. $\varepsilon 8$.

trigonum Schr. 186.

virgatum Koch. 88.

Epipactis atrorubens Hoftm. 129.

ensifolia $\mathrm{Sw}, 129$.

grandiflora Gaud. 129.

lancifolia DC. 129.

latifolia All. 44, כัl , 129.

a pallens Gaud. 129.

$\beta$ rubiginosa Gaud. 129.

microphylla Sw. 188.

ovata All. 130.

pallens Sw. 129.

palustris Crantz. 45, 51, 129.

rubiginosa Gaud. 14, 51, 129.

rubra All. 150.

Epipogium Gmelini Rich. 188.

Equisetum fluviatile Sm. 145.

hyemale L. 51. 146.

$\beta$ paleaceum Doel. 17, 146.

limosum L. $51,146$.

multiflorum $\alpha$ Vauch. 146.

palustre L. 51, 146.

sylvaticum L. $48,146$.

Telmateya Ehrh. 28, 145.

variegatum Sch]. 54, 146.

Erica vulgaris L. 107.

Erigeron aipinus L. 48, 97.

ghabratum Hopp. 186. 
Lrints alpinus L, 51, 37, 113.

Eriophorum alpinum L. 188.

angustifolium Roth. 28, 134.

vaginatum L. 41, 154.

Erodium triviale Jord. $58,75$.

Erophila verna DC. 54, 68 .

glabrescens Jord. 54.

majuscula Jord. 34 .

Erucastrum obtusangulum Reich. 53, 68.

Eryngium alpinum L. 186.

Erysimum ochroleuenm DC. 185.

officinale L. 68 .

orientale R. Br. 67.

Erythraea centaurium Pers. 56, 110.

Erythronium Dens eanis L. 22, 152.

Euphorbia amygdaloïdes L. $50,121$.

cyparissias L. 121.

dulcis L. $50,121$.

exigua L. 122.

falcata L. 122.

helioscopia L. 122.

peplus L. 122.

platyphyllos L. 122.

stricta L. 122.

sylvatica Jacq. 121.

verrucosa L. $\beta$ montana Gaud. 188.

Euphrasia alpina DC. Gaud. 114.

campestris Jord. $56,114$.

cupraea Jord 56, 114.

ericetorum Jord. 55, , $56,114$.

minima Jacq. 187.

montana Jord. 45, 114. 
Firplirasia nitidula Reut. 187.

odontites Koch. 114.

officinalis L. $\beta$ montana Rap. 114.

$\gamma$ campestris Rap. 114.

Salisburgensis Funk. 5้6, 114.

$\beta$ cнpraea Rap. 114.

uliginosa Ducom. 114.

verna Bell. $1 / 4$.

Eronymus Europaeus L. 29, 45, 77.

lati olins L. $16,46,77,158$.

Faba vulgaris Moench. $3 z, 80$.

Fagus sylvatica L. 122.

Fedia auricula Gaud. 96.

dentata Gaud. 96.

olitoria Gand. 96.

Festuca ciliata Pers. 140.

decumbens L. 140.

duriuscnla L. $\beta$ glauca Rap. 141.

elatior L. 141.

gigantea Vill. 5้2, 141.

glanca Lam. 42, 49, 141.

heterophylla Lam. 141.

myuros Pol. Gaud. 140.

myuros Reich. 140.

nigrescens Lam. 189.

ovina L. $55,140$.

$\gamma$ tenuifolia Dub. 140.

pratensis Huds. 5ั0, 141.

pseudo-myuros S. W: 140.

pumila Vill. 189. 
Festuca rigida Kunth. Koch. 140.

Scheuchzeri Gaud. 189.

sylvatica Vill. $47,141$.

tenuifora Schr. Koch. 141.

tenuifolia Sibt. 52, 140.

Ficaria ranumeuloides Mœnch. 22, 55, 63.

Filago arvensis L. 5้4. 99.

canescens Jord. 99.

Gallica L. 5ั4, 99.

Germanica L. 5ั4, 9!).

$\beta$ pyramidata Gaud. 99.

Jussiaei C. et G. 99.

lutescens Jord. 99.

minima L. 15, 5ั6, 99.

montana L. Gaud. 99.

spathulata Presl. 54, 99.

Foeniculım officinale All. $50,91$.

Fragaria collina Ehrb. 45, 82.

dumetorum Jord. 186.

vesca L. 43.82.

Fraxinus excelsior L. 24, 109.

Fumaria Chaviui Reut. 32, 56, 65.

officinalis L. $5 \overline{5}, 65$.

Vaillantii Lois. Rap. 65.

Gagea arvensis Schultz. 25, 152.

lutea Schultz. 35 , 36, 132.

$\beta$ sylvatica Dub. 132.

villosa Dub. 132.

Galeobdolon luteum Huds. 30, 116.

Galeopsis angustifolia Ehrh. 50, 55, 116 .

intermedia Vill. 40, 49, 116.

Ladanum Autet, non Lin. 116. 
Galeopsis Ladanum II latifolia Gaiid. 116.

praecox Jord. 38, 116.

Reichenbachii Reut. 49, 116

tetrahit L. $\beta$ praecox Rap. 116.

$\gamma$ Reichenbachii Rap. 116.

Galium anisophyllum Vill. 186.

Boccone All. 95

boreale L. 51, 95.

commutatum Jord. 44, 958.

elongatum Presl. 51.

glaucum L. 37, 95 .

montanum Vill. 45, 95.

spurium L. 95.

$\beta$ Vaillantii Gr. et $G_{*}, 38,93$.

$\gamma$ tenerum Gr. et G., 15, 34, 95, 170.

sylvaticum L. 44, 51, 95.

sylvestre Poll. 44, 95.

$\alpha$ laeve Thuil. 95.

$\beta$ Boccone All. 95 .

tenerum Schl. Gaud. 93.

tricorne With. $\mathbf{3 8}, 95$.

uliginosum L. 54, 95.

vero-mollugo Wallr. 191.

Cenista Germanica L. $29,77$.

pilosa L. 29, 77.

sagittalis L. 48, 79 .

Gentiana amarella Pol. Gaud. 110.

campestris L. 5๊ 110.

Centaurium L. 110.

Charpentieri Thom. 192.

ciliata L. 56, 110.

Clusii Per, et Song. 187.

cruciata L. 55, 110. 


\section{Xxi}

Ger tiana Germanica Willd. 56, 110

lutea L. 18, 110.

pneumonanthe L. 187.

punctato-lutea Gris. 192.

purpureo-Intea Gris. 192.

Thomasii Gill. 192.

verna L. $3 \overline{5}, 110$.

Geranium cicutarium L. 75 .

columbinum L. 58, 75

dissectum L. 58, 75 .

lucidum, L. $\mathbf{5 5}, 75$.

molle L. 58,75 .

palustre L. 47, 75 .

phaeum Lam. 185.

pusillum L. $38,75$.

pyrenaicum L. $\mathbf{3 8}, \mathbf{7 5}$.

Robertianum L. 38, 7 \%.

rotundifolium L. 38,75 .

sanguineum L. 44, 75.

sylvaticum L. 40, 42, 48, 73.

Geum montanum L. 42, 81, 159.

rivale $\mathrm{L}$, 41, 81 .

urbanum L. 81 .

Glechoma hederacea L. 55, 116.

Globularia cordifolia L. 30, 117.

nudicanlis L. 52, 118.

vulgaris L. $\mathbf{3 0}, 118$.

Glyceria plicata Fries, 4T, 159.

Guaphalium dioicum L. 40, 99.

rectum Sm. 99.

supinum L. 186.

sylvaticum L. $55,99$. 
Goodyera repens R. Br. 52, 150.

Gymnadenia albida Rich. 48, 128.

conopsea Rich. 128.

odoratissima Rich. 128.

Gypsophila repens L. 185.

vaccaria Sibt, et $\mathrm{Sm} .38,71$.

IIedera Helix L. 93.

Heleocharis acicularis $\mathbf{R}$. $\mathrm{Br} .154$.

Helianthemum canum Dun. 50, 70).

Fumana Mill. 44, 69.

grandiflorum DC. 185.

Elandicum Wahl. $\beta$ canum Rap. 70.

pulverulentum DC. 185 .

vulgare Gaertu. 50.

$\beta$ hirsutum Koch. 70.

$\gamma$ grandiflorum Koch. 70.

Helleborus foetidus L. $22,63$.

viridis L. $22,63,152$.

Hepatica triloba DC. 20, 22, 62.

Heracleum asperum Koch.92.

montanum Schl. Gaud. 52, 92.

panacea L. 91.

Sphondylimm L. 48, 95.

Herminitn monolehis R. Br. 55, 102, 129

Hesperis Alliaria L. 68.

Hieracium amplexicaule L. こั()

$\zeta$ aureum Gaud. 105.

\& pseudo-cerinthe Gaud. 103.

$\gamma$ pulmonarioides Gaud. 105.

andryaloides Vill. 15, 30, 103, 174.

aurantiacum L. 187. 


\section{XXIII}

Hieracium aureum Scop. 102.

auricula L. forma alpina, 40, 48, 102.

boreale Fries. 51, 104.

var. curvidens Gr. et G. 56.

var. dumosum Gr. et $\mathrm{G}$. 104 .

var. vagum Gr. et $G .104$.

var. virgultorum Gr. et G. 104.

caesium Fries. 42, 103.

Cerinthoides Vill. 105.

dentatum Hopp. 187.

$\beta$ Salaevense Rap, 48, ร้อ̈, $105 .$.

dubium Gaud. 102.

elatum Fries. 48, 104.

$\beta$ Laggeri Reut. 187.

elongatum Willd. 187.

fallax Gaud. 102.

Hexuosum W. et K. 44, 55, 102.

$\beta$ calvum Gr. et $G .187$.

Florentinum All. 51, 102.

glabratum Koch. Gr. et G. 102

glaucum All. 44, 522, 5้3, 102, 175

hybridum Gaud. 102.

Jacquini Vill. $51,103$.

lanatum Vill. 15, 52, 105, 174.

Ligusticum Fries. 48, 50 , ร̌ , 105 .

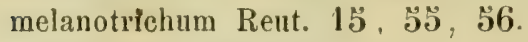

murorum L. $50,105$.

๔ nemorense Rap. 103.

$\gamma$ praecox. Rap. 103.

$\delta$ coesium Rap. 105.

nemorense Jord. $30,51,105$.

paludosum L. 102. 


\section{XXIV}

Hieracium pilosella L. 102.

pilosello-praealtum Schultz. 11, $\mathbf{1 0 2}, 192$.

piloselloides Vill. 102.

porrectum Fries. 187.

praealtum Gaud. 102.

praealtum Vill. 15, 102.

praecox Schultz. 50, 103.

psendo-cerinthe Koch. $103,48,49,105,176$.

prenanthoides II Juranum Gaud. 104.

pulmonarioides Vill, 48, ๖0, 55 .

rigidum Hartm 104.

Sabaudum Gaud. Rap. 104.

Sabaudum L. $\varepsilon$ vagum Rap. 104.

staticefolium Vill. 44, 102.

sylvestre 'Tausch. 104.

tridentatum Fries. כ̌6, 104.

umbellatum L. ร้อ๊, 104.

vagum Jord. 56,104 .

villosum L. 42, ร้5ี, 103.

Vogesiacum Moug. 187.

Hippociepis comosa L. 50, 79.

Hippophae rhamnoides L. 24, 55, 121.

Homogyne alpina Cas. Koch. 48, 97.

Hordeum distichum L. 142.

hexastichum L. 142.

muriuum L. 142.

vulgare L 58, 112.

zeocriton L. 58, 112.

Intchinsia petraea R. Br. $26,51,67$.

alpina $\mathrm{R}, \mathrm{Br} .18 \%$.

Hyacinthụs comosus L. 155.

racemosus L。 153. 


\section{XXV}

Hypericum dubium Leers. 74.

fimbriatum L. 185 .

hirsutum L. 74.

lumifusum L. 54, 74.

$\beta$ Liottardi DC. 56, 74.

montanum L. $51,71$.

perforatum L. $7 \mathfrak{f}$.

pulchrum L. 74.

quadrangulum L. $48,74$.

tetrapterum Fries. 74.

Hypochaeris maculata L. 40, 100.

Iberis amara L. 54, 57, 67.

ceratophylla Reut. 185.

pinnata L. 54, 67.

Ilex aquifolium L. 50, 44, 109.

Impatiens noli-tangere L. 47, 75 .

Inula pulicaria L. 98.

semi-amplexicaulis Reut. 191.

Isnardia palustris L. 56,89 .

Jasione montana L. 50, 105.

Juglans regia I. 122.

Juncus alpinus Vill. 155.

glaucus Ehrh. 155.

inflexus Leers. 135.

obtusiflorus Ehrh. 51, 153.

Juniperus communis L. $30,125$.

nana Willd. 188.

Kernera saxatilis Reich. $51,67$.

Knautia sylvatica Dub. 5้š, 96. 
Koeleria cristata Pers. 48, 159.

Lactuca dubia Jord. 500, 101.

muralis Fres. 45, 59. 101.

perennis L. 187.

scariola Gaud. 101.

Lamium amplexicaule L. 25, 117 .

maculatum L. 25, 117.

purpureum L. 25, 117.

Lappa intermedia Reich. 5ร5, 100.

major Gaertn. อ๊อ๊, 100.

pubens Bor. 187.

tomentosa Lam. 187.

Larbraea aquatica St-Hil. 72.

Larix Europaea DC. 188.

Laserpitium latifolinm L. รั3, 92.

$\beta$ asperum Gaud. 186.

pruthenicum L. 51 , 54, 92.

siler L. 53 , 92.

Lasiagrostis calamagrostis Link. 37, 138.

Lathraea squamaria L. 24, 112.

Lathyrus heteropliyllus L. 183.

hirsutus L. 37, 79.

pratensis L. 44, 79.

sylvestris L. 44, 79 .

Leontodon aureum L. 102.

autumnale L. 5ૅ马, 100.

hastile L. 44, 53 , 101.

hispidum L. 40, 101.

taraxacum L. 101.

Leontopodium alpinum Cass, 186.

Lepidium campestre L. 34, 68 .

petraeum L. 67. 
I 4 pucanthemum corymbosum Gr. et G. 44, 51, ?8.

montanum Koch. 44, อั5.

vulgare Lam. 44, 104.

Lencoinm vernum L. 20, 22, $34,151$.

Libanotis montana All. 186.

Ligusticum ferulaceum All. 186.

Ligustrum vulgare L. 44, 109.

Lilium Martagon L. 45, 44, 132.

Limodorum abortivnm Sw. 45, 129.

Linaria alpina Mill. 112.

cymbalaria Mill. $48 \%, 112$

elatine Mill, 50, 112.

minor Desf. 58, 50, 112.

petraea Jord. 187.

spuria Mill. $50,112$.

Linum alpinum L. Jacq. $\beta$ montanum Reut. 185.

catharticum L. 44, 75 .

tenuifolinm L. $44,73$.

usitatissimum L. $\mathbf{3 8}, 7 \bar{\jmath}$.

Liparis Loeselii Rich. 45, 150.

Listera cordata Rich. 188.

ovata R. Br. 150.

Lolium perenne L. 58, 142.

rigidum Gaud. 141.

strictum Presl. 58, 141.

temulentum $\mathrm{L}$. $\mathbf{3 8}, \mathbf{1 4 1}$.

Lonicera alpigena L. 30, 44, 94.

coerulea L. 186.

nigra L. 50, 32, 44, 94.

periclymenum L. 44,94 .

xylosteum L. 50, 44, 94.

Lotus corniculatus L. 41, 44, 49, 79.

var. tennifolins Gand. 78. 
Lohns tenuifolius Reich. 78.

tenuis Kit. 45, 78.

Lmuria rediviva L. $51,67$.

Luzula flavescens Gaud. 42, 154.

maxima DC. 44, 49, 134.

multiflora Lej. $\beta$ congesta Gr. et G. 188 .

$\gamma$ nigricans $\mathrm{Gr}$. et $\mathrm{G} .188$.

nivea DC. 42, 44, 154.

spicata DC. 188.

sylvatica Gaud. 154.

Lyclınis diurna Sibt. 42, 71.

flos-cuculli L. 25, 71.

Githago Lam. 71.

sylvestris Hopp. Gaud. 71.

Lycopodium annotinum L. 189.

clavatum L. $15,49,155$.

salaginoides L. 145.

selago L. 49, 145.

Lysimachia nemorum L. 42, 48, 109.

Lythrum lyyssopifolia L. 89.

Maianthemum bifolium DC. 50, 131.

Malaxis Loeselii Sw. 150.

Malus acerba Mer. 26, 88.

communis Poir. 26, 29, 88.

Malva rotundifolia L. 54, 73.

sylvestris L. 54, 7.3.

Matricaria chamomilla L. 46, 98.

Margarita Bellidiastrum Gand. 97.

Medicago sativa L. $\mathbf{3 8}, \mathbf{8 0 .}$

falcato-sativa Reich. 191.

Iupulina L. 38, 72.

media Pers. 191. 


\section{XXIX}

Melampyrum pratense L. 45, 115.

sylvaticum L. 48, 113.

Melica ciliata L. Gaud. 139.

Nebrodensis Parl. Gr. et G. 44, 159.

nutans L. 44, 159.

uniflora Retz. 44, 159.

Melilotus arvensis WVallr. 58, 78.

officinalis Lam. 78.

Mentha aquatico-arveusis Wirtg. 192.

aquatico sylvestris Mey. 192.

arvensi pulegium Reut. 192.

candicans Crantz. 52, $11 \%$.

hirsuto-arvensis Rap. 192.

nepetoides Lej. 192.

rotundifolia L. $50, \mathbf{1 5}$.

sylvestris L. $52, \mathbf{1 1 5 .}$

sylvestri-rotundiolia Wirtg. 192.

viridis L. 52, 115.

var. canescens Gr. et G. 11 s.

Menyanthes trifoliata L. 4\%, 109

Mercurialis annua L. 122.

perennis L. 25, 122.

Mespilus Amelanchier L. 87.

cotoneaster L. 87.

Germanica L. 38, 87.

oxyacantha DC. 86.

tomentosa Gand. 87.

Milium effusum L. 44, 48, 158.

Moehringia muscosa L. 31, 72.

trinervia Clairv. 42, 72.

Molinia coerulea Moench. $5 \mathbf{1}$.

$\beta$ altissima Gcd, 140.

littoralis Host. 51, 140. 
Monotropa hypopitis L.

a glabra God. $55,108$.

$\beta$ hirsuta Gol. 5๊, 108.

Mulgedium alpinum Less. 187.

Muscari comosum Mill. 51, 155. racemostum Mill. 95, 152.

Myagrum sativum L. 67. saxatile L. 67.

Myosotis alpestris Schultz. 187.

lappula L. 111.

perennis $\beta$ sylvatica DC. 110 . sylratica Elıh. 52, 49, 110.

Myricaria Germanica Desv. 35, 89.

Myrrhis odorata Scop. 48, 93, 169.

Nareissus bifloris Curt. $56,1 / 4$. poeticns L. $56,151$.

Psendn-Narcissus L. 22, 151.

radiiflorus Salisb. 188.

Nardurus tenellus Reich. 55, 57, 141.

Nardus stricta L. $40,48,141$.

Neottia aestivalis DC. 150.

nidus-avis Reich. 150.

repens DC. 150.

spiralis DC. 130.

Neslia paniculata Desv. 34, 68.

Nigella arvensis L. $46,65,155$.

Nigritella angustifolia Rich. 40, 49, 129.

nigro-conopsea Reich. 49, $129,192$.

nigro odoratissima 192.

suaveolens Koch. 188, 192 
Nitella capitata Agr. $51,146$.

glomerata Ag. 5ั1, 146.

opaca Ag. 51, 146.

polysperma A. Br. $31,146$.

Nymphaea alba L. 45, 64.

Odontites Intea Reich. 187.

rubra Gr. et G. 50, 114.

verna Reich. 114.

Enanthe Lachenalii Gm. 5้), 91.

pencedanifolia Gaud. 91.

Onobrychis montana DC. $18 \%$.

sativa Lam. DC. 80.

vulgaris Gaud. 80.

Ononis rotundifolia L. 15, 29, 54, 78, 157.

Ophrys apifera Huds. 50, 129.

arachnites Reich $70,129$.

aranifera Huds, 50, 128.

Corallorhiza L. 130.

fucifera Reich. God. 129.

insectifera I. 128.

Loeselii L. 150.

monorchis $\mathrm{L}$. 129.

muscifera Huds. 50, 128.

myodes Jaeq. Gaud. 128.

nidus-avis Rich. Gand. 150.

ovata L. 150.

Orchis abortiva L. 120.

albida All. 128.

angustifolia W. et G. Knch. 127 ..

bifolia L. 45, 128.

$\beta$ elatior, Gaud. 128. 
Orchis conopsea L. 45, 49, 52, 128. coriophora L. $36,127$. fusea Jacq. 127. galeata Lam. 50, 127. globosa L. 40, $48,127$. incarnata L. 28, $56,127$.

$\beta$ angustifolia Reich. Gr. et G. 127. latifolia L. Koch. 28, 56, 127. maculata L. 128. maialis Reich. 127. mascula L. 50, 127. militaris $\alpha$ L. Gaud. 127. morio L. $50,127$. morio-laxiflora Reut. 192 nigra All. 40, 129. nigro-conopsea Reich. 129. odoratissima L. 45, 52, 128. purpurea Huds. $50,127$. purpureo-militaris Gr. et G. 192. pyramidalis L. 128.

Sambucina L. 42, 127. Simia Lam. 50, 127. simio-purpurea Wedd. 192. Traunsteineiri Saut. 45, 127. ustulata L. 50, 127. vireseens Zollik. 45. 128. viridis All. 128.

Origanum vulgare L. 58, 115.

Orlaya grandiflora Hoffm. 57, 51, 92.

Ornithogalum arvense Pers. 152.

luteum $\beta$ L. 152.

minimum DC. 152.

pyrenaicum L. 44, 132 
Ornithogalum sylvaticum Pers. 152.

Orobanche brachysepala Schultz. 112.

Cervariae Suard. 58, 50, 112.

Epithymum DC. 187.

Galii Dub. \%5 111.

Laserpitii-sileris Rap. 55, 111.

ramosa L. 111.

Scabiosae Koch. 5ั5. 111.

'Tenerii Schultz, Rent. 57, 53, 111.

Orobus luteus L. 185.

niger L. 50) 79.

tuberosus L. $50,79$.

vermis L. $25,79$.

Osmunda Spicant L. 145.

Oxalis acetosella L. $23,76$.

Europaea Jord. 56, 76.

stricta anct. 76 .

Oxyeoceos vulgaris Dun. 187.

Oxytropis montana: DC. 18 sั.

Panicum crus-Galli ¿. 142.

glabrum Gaud. 157.

glancum Gaur. 157.

sanguinale L. 142.

Papaver argemone L. 58, 64.

dubinm L. 57, 64.

a Lecoeqii Lamot. 57, 64.

$\delta$ collinum Bog. 57, 64 .

rhoeas L. 38, 64.

Paradisia Liliastrum Bert. 188.

Parictaria erecta M. et K. 44, 129.

officinalis L. $25,50,151$.

Parmassia palustris L. 41, 51, 69. 
Pedicnlaris foliosa L. 187.

$$
\begin{aligned}
& \text { palustris L. } 45,115 . \\
& \text { tuberosa L. } 15,49,115,180 .
\end{aligned}
$$

Peplis "portula L. ๖̌4, ๖6, 89.

Petasites albus Gaertn. 186.

niveus Baumg. 186.

officinalis Mœnch. 22, 24, 97.

Reuteriana Jord. 24, 97.

vulgaris Desf. 97.

Peucedanum Cervaria Lap. Koch. §4, 92.

Chabraei Gand. 186.

Oreoselinum Mœnch. 186.

Silaus L. 95.

Phaenixopus muralis Koch. 101.

Phalangiım Liliago Schr. 155.

ramosum Lam. 155.

Phalaris arundinacea L. Gand. 156.

phleoides L. 157.

Phaseolus nanus L. 55, 80.

vulgaris L. $\mathbf{3 3}, 80$.

Phegopteris alpestris Mett. Bern. 189.

calcarea Fée. Bern. 577, 145.

Dryopteris Fée. 145.

Phelipea ramosa C.A.Mey. ๖/, 111.

Phleum alpinum L. 48, 157.

$\beta$ foliosum Reut. 189.

$\gamma$ commutatum Rent. 189.

Boehmeri Wib. 57, 157.

Michelii All. 189.

nodosum L. 156.

phalaroides Kael. Gaud. 157.

pratense L. 40.

$\gamma$ nodosum Gaud. 40, 136. 
Phyteuma orbicularis L. 187.

spicata L. Horo cœruleo. $59,1030$.

Picris crepoides Sant. 187.

Villarsii Jord. 187.

Pimpinella dioica L. 91.

magna L. 40, 91.

B rosea Koch. 40, 48, 91.

saxifiaga L. 53, 91.

l'inguicula alpina L. รั2, 117.

grandiflora Lam. 188.

$\beta$ pallida Gand. 188.

vulgaris L. $28,117$.

Yinus abies L. 125.

picaea L. 125.

sylvestris L. $50,125$.

$\delta$ pumilio Gaud. 125.

uncinata Ram. 30, 123.

Pisum arvense L. 35, 80

sativum L. 55, 80.

Plantago alpina L. 49, 118.

cynops L. 16, 45, 118, 180.

integralis Gaud. 118.

lanceolata L. 118.

major L. 50, 118.

media L. $50,118$.

montana Lam. 188.

serpentina Vill. 16, 52, 118, 180.

Wulfenii Koch. 118.

Platanthera bifolia Rich. 128.

chlorantha Cust. Reich. 128.

viridis Lindl. 48, 128.

Poa alpina L. 40, 48, 159.

$\beta$ brevifolia Gr. et $\mathrm{G} .42,49,159$. 


\section{xxxy}

Poa annua L. 50, 159.

coaretata DC. 139.

fertilis Host. 189.

liybrida Gaud. 189.

nemoralis L. 159.

$\beta$ rigidula Gaud. 44, 48, 159.

pratensis L. 142.

rigida L. 140.

Sudetica Haenk. 189.

trivialis L. 142.

Polycnemum arvense L. 54, 119.

$\alpha$ majus Rap. 119.

majus A. Br. 5̌4, 119.

Polygala alpestris Reich. 48, 69, 156.

amara L. Koch. 41, 69.

$\beta$ alpestris Koch. 69.

$\beta$ alpina Gaud. 69.

$\gamma$ austriaca Koch. 69.

Austrica Crantz. 4: , 69.

Chamaebuxus L. 20, 29, 69.

comosa Schr. 44. 69.

uliginosa, Reich. 69.

vulgaris L. 44, 69.

Polygonum bistorta L. 188.

fagopyrum L. $50,120$.

lapathifolinm L. $\gamma$ incanum Reut. 54, 120.

viviparum L. 188.

Polypodium aculeatum Doel. Koch. 144.

calcareum Sm. 57, 145.

dryopteris L. 145 .

filix-Foemina L. 144.

filix-Mas L. 144.

fragile $L .144$. 
Polypodium fragrans Vill. 144.

Lonchytis L. 145

rigidum Hoffin. 58 .

Robertianum Hoftm. 145.

Thelipteris L. 144.

vulgare L. 57, 145.

Polystichum aculeatum L. 144.

filix-Mas Rotl.. $57,144$.

Oreopteris DC. 189.

rigidum $\mathrm{DC}$." 144.

spinulosum DC. 57, 144 .

'Thelipteris DC. Roth. 57, 144.

Populus alba L. 21, 124.

tremula L. $21,124$.

Potamogetum densum L. $\beta$ lanceolatum Koch.

รั5 , 126.

Hornemanni Mey. 126.

plantigineus Ducr. 45. 126.

rufescens Schr. 188.

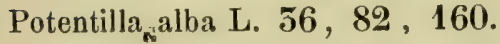

alpestris Hall. fil. 82, 186.

aurea Gaud. 82.

aurea L. Koch. 41, 82.

caulescens L. 47, 82, 160.

Halleri Ser. 82.

intermedia L. 186.

Jurana Reut. 41, 82.

minima Hall. fil. 186.

petiolulata Gaud. 15, 51, 52, 82, 161 .

rupestris L. 54, 82, 160.

Salisburgensis Haenk. 82.

tormentilla Nest. 41, 82.

verna $L .41,82$. 
Prenanthes muralis L. 101. purpurea L. $400,55,101$.

Primula acaulis Jacq. 25. acauli-elatior. Mur. 192. acauli-officinalis Mur. 23, 108.

acauli-suaveolens, 23, 192.

auricula L. 46, 108, 178.

elatior Jacq. 32, 108.

farinosa L. 56, 108.

grandiflora Lam. 22, 108 .

officinalis Jacq. 22, 26, 108.

$\beta$ suaveolens Rap. 108.

officinali-acaulis Mur. 25, 108, 192.

officinali-elatior Mur. 192.

suaveolens Bert. 22, 108.

variabilis Goup. 22, 23, 26, 108, 192.

veris $L .108$.

$\beta$ elatior L. 108.

$\gamma$ acaulis L. 108.

Prismatocarpus speculum L'her. 105.

Prunella grandiflora Jacq. 43.

laciniata Jacq. $4 \mathbf{5}$.

Prunus avium L. 80.

fruticans Weih. 203,80 .

Mahaleb L. 80.

Padus L. 80.

spinosa L. 25, 80.

$\operatorname{var} \beta$ Koch. 80.

Pteris aquilina L. 15, 57, 145.

Pulicaria vulgaris Gaertn. 54, 98.

Pulmonaria angustifolia Gaud. 110.

officinalis L. 25, 110.

tuberosa Schr. 25, 110. 
Pyrolat chloranta Sw. 47, 107, 177.

media Sw. 15, 52, 107, 177.

minor L. 48, 107.

rotundifolia L. 52, 107.

secunda L. 52, 107.

uniflora L. 187.

Pyrus acerba DC. 88.

Aria DC. 88.

Aucuparia Crantz. 88.

communis L. 88.

intermedia DC. 88.

Malus L. 88.

Sorbus Gaertn. 87.

torminalis DC. 88.

Quercus pedunculata Ehr. 125.

pubescens Willd. 50. 122.

racemosa Lam. 50.

sessiliflora Sm. 30, 125.

Ranunculus aconitifolius L. 41, 48, 62 .

acris L. $38,48,65$.

arvensis L。 38 .

bulbosus L. 38, 65.

divaricatus Schr. 45, 62.

Drouettii Schr. 56, 62 .

Ficaria L. 65.

gracilis Schl. 53, 41, 62.

lanuginosus L. 48, 63.

montanus Willd. 63.

nemorosus DC. 42, 62.

paucistamineus Tausch. $36,62$.

platanifolius L. $11,62$. 
Ramunculus repens L. 58, 65 .

Thora L. 41, 19, 62.

trichophyllus Chaix, 31.

Raphanus raphanistrum L. 54, 68.

Rapistrum rugosum All. 54, 68.

Reseda lutea L. 69.

phyteuma L. $57,69,45$.

Rhamnus alpinus L. $29,52,77$.

catharticus L. $29,77$.

frangula L. $29,77$.

Rhinanthus alectorolophus Poll. 58, 114.

angustifolius $\mathrm{Gm}$. 11, 49, 114 .

crista-Galli L. a 114.

$\beta$ L. 114.

$\gamma$ L. 114 .

major Elırh. 44, 114.

minor Elır. 114.

Rhododendron ferrugineum L. 187.

Ribes alpinum L. 29, 90.

grossularia L. $\beta$ pubescens Koch. 90.

petraeum L. 186.

uva crispa L. $25,90$.

Rosa alpestris Rap. 15, 40, 48, 84.

alpina L. $59,45,84$.

$\beta$ pyrenaica DC. 59, 84.

$\gamma$ lagenaria Vill. 59, 84.

$\delta$ aculeata Ser. 59, 84.

alpino-pimpinellifolia Reut. 45̃, 85, 191.

Andegavensis Bast. 45, 44, 855 .

arvensis Huds. 45, 86.

Austriaca Crantz, 56.

biserrata Mer. 40, 85 . 
Rosa canina L. 4z, 44.

$\beta$ senticosa God. 45, 83.

$\gamma$ sarmentosa God. 45,85

$\delta$ collina God, 45, 85.

Chavini Rap. 44, 84, 191.

comosa Rip. 85.

coriifolia Fries, 40, 45, 84.

var. biserrata Reut. 40, 84.

coronata Crep. 15, $59,84$.

foetida Bast. 46, 47, 85 .

Gallica L. 45, 86.

Gallico-arvensis, 86, 191.

Gallico-canina Reut. 45, 86, 191.

Gallico-umbellata Rap. 45, 86, 191.

glandulosa Bell. 84.

glanca Vill. 84.

hybrida Schl. 45, 86, 191.

inodora Fries. 85.

Kluckii Bess. 85.

marginata Walr. 13, 45, 84 .

micrantha Sm. 45,83 .

mollissima Fries. 57, 45, 84.

montana Chaix. 45, 52, 85, 165.

monticola Rap 4 5.

$\beta$ alpestris Rap. 84.

$\varepsilon$ cinerea Rap. 40, 84 .

pimpinellifolia L. 45.

$\alpha$ vulgaris God. 55, $8 \tilde{5}$.

$\beta$ spinosissima God. 44, 85 .

pimpinellifolio-alpina Rap. 41, 85, 191.

pumila Jacq. 86.

psilophylla Rau. Desegl. 86.

Renteri God. 45, 84, 165. 
Rosa var. foliis biserratis, 82 .

rubiginosa L. 45, 85 .

a parvifolia God. 85 .

var. nemorosa Reut. 45, 85 .

var. sepium God. 83 .

rubrifolia Vill. 40, 43, 84.

var. montana Ser. 85.

Sabauda Rap. 15, 40, 45, 84, 163.

$\beta$ coronata Rap. 84 .

Salaevensis Rap. 15, 40, 45, 84, 162.

sepium Thuill. 44, 45, 85.

$\delta$ pubescens Rap. Reut. 44, 85.

sphaerica Gren. 40, 44, 85.

spinulifolia Dem. 43, \$4.

subglobosa Sm. 57, 45, 84 .

tenuiglandulosa Mer. 85 .

tomentella Lam. 57, 行, 83.

tomentosa Sm. $\mathbf{4 4}, \mathbf{4 5}, \mathbf{8 4}$.

$\beta$ marginata Rap. \$4.

$\gamma$ subglobosa Rap. 84.

$\gamma$ dumetorum Gaud. 83.

umbellata Leers. $45,83$.

vestita God. 15, 40, 45, 84, 162.

Rubus albidus Merc.

$\beta$ Salaevae Merc. 59, 49, 81.

Bellardi W. et N. 185.

coesius L. 59, 45, 81 .

caesio-Idaeus Merc. 45, 81, 191.

collinus DC. $15,45,81,161$.

conspicuus P.-J. Mull. 186.

cuneifolius Merc. $4 \mathbf{5}, \mathbf{8 1}$.

discolor W. et N. $43,49,81$. 
Rubus dumetorum W. et N. 3981.

a virescens Merc. 59.

elongatus Merc. 59, 49, 81.

Guntheri W. et N. $39,49,81$.

hirtus W. et N. $59,49,81$.

hispidus Merc. 186.

Idaens L. $45,81$.

Idaeo-coesins Merc. 194.

nemorosus Gr. et G. 45.

patens Merc. 194.

psendo-coésius Merc. 191,

rigidus Mere. 186.

rudis W. et N. 185.

rusticanus Merr. $59,45$.

saxatilis L. 50,81 .

tomentosus Borck. 186.

vestitus, IV. et N. 186.

Rumex acetusa L. 50, 120.

acetosella L. 44, 120.

arifolius All. $52,120$.

conglomeratus Murv. 50, 120.

crispus L. $ّ 0,120$.

obtusifolius L. 500, 120.

pulcher L. 50, 120.

scutatus L. 188.

Ruscus aculeatus L. 26, 152.

Sagina Linnaei Presl. 41, 48, 72.

nodosa Fenzl. 185.

procumbens L. 42, 71 .

Salix acuminata Hoffm. 124.

alba L. 21, 125.

anbigua Ehru. 188. 
Salix amygdalina L, 21. 125.

a discolor God. 21, 125.

B concolor God. 21, 125.

aurita L. 21, 124.

capraea L. 21, 124.

cinerea L 21, 124.

cinereu-nigricans Reut. 192

daphoides L. 21, 27, 125.

grandifolia Ser. 24, 52, 12/.

incana Sehr. 27, 124.

incano-capraea Ćlav. 125, 192.

incano-daphnoides Wim. 192.

lanceolata Ser. 125.

lavandulaefolia Lap. 121.

nigricans Fries, 124.

var. eriocarpa God. 26, 5i, 124.

var. leiocarpa God. 27, 124.

pentundra L. 188.

phylicifolia Wahl. 12/.

Pontederana Willd. Gand. 21, 26, 125, 192

praceox Hopp. 125.

purpurea L. 27, 125.

purpureo-cinerea Rap. 125, 192.

purpurco-daphnoides Chas. 125192.

repens L. $56,124$.

peticulata L. 188.

retinsa L. 188.

riparia Wild. 124.

Seringeana Gand. 26, $125,192$.

Smithiana $\beta$ obseura Gr. et $G$. 125.

triandra L. 125.

Wimmeri Kern. 192.

Salvia glutinosa $\mathrm{I}$. ฏ้อ 115. 
Salvia pratensis L. 115. verticillata L. 187.

Sambucus ebulus L. כ้1, 94.

nigra L. 50, 94.

racemosa L. 29 , 52, 94.

Sanguisorba montana Jord. Rent. $40,86$.

officinalis L. 86.

serotina Jord. Reut. 86 .

Sanicula Europaea L. 45, 91.

Saponaria vaccaria L. 71 .

Satyrium repens L. 150.

Saxyfraga aizoides L. らૅ, 90.

aizoon L. 42, 90.

cotyledon var. $\varepsilon$ L, 90.

granulata L. 51, 90

Hirculus L, 186.

muscoides Wult. 186.

mutato-aizoidss Rent. 191.

oppositifolia I. 186.

rotundifolia L, 90.

tridactylites L. 51, 90.

Scabiosa lucida Koch. 186.

succisa L. $5 コ$, 96.

sylvatica I. 96.

Seandix anthriscus L. 95.

cerefolium L. 95.

pecten-Veneris L. 58, 95.

Schenchzeria palustris L. 188.

Schoenus compressus L. 154 .

nigricans $L .45,45,154$.

Scilla bifolia L. 20, 22. 152.

Scirpus acicnlaris 1. 154.

Baeothryon Ehr. Gand, 154. 


\section{XLVI}

Scirpus coespitosus L. 188.

compressus L. 45, 154.

Halleri Vill. 154.

pauciflorus Light. 45, 45, 134.

setaceus I. 15/.

Scleranthus aunuus L, $\mathbf{5} 6$.

biennis Rent. 13, 55, 41, 48, 89.

Delorti Bill. 89.

perennis I. $15,36,42,47,89,168$.

verticillatus Reich, 58, 89.

Scleropoa rigida Griseb. 57, 140 .

Scolopendrium officinale Sm. ร̌7, 148.

Sconzonera Austriaca Willd. 31, 101. 175.

humilis L. 51, 101, 172.

humilis Jacq. DC. non L. 101.

plantaginea Schl. 101.

Serophularia squatica Gr. et G. non L, 112.

Balbisii Horn. 5้0, 112.

Juratensis Schl. 187.

Secale cereale L. 58, 142.

Sedum

album L. $55,90$.

anopetalum DC. 15, 52, 90, 169.

atratum L. 186.

dasyphyllum L. 555, 90

maximum Koch. כ06, 89.

reflexum L. 52, 50.

$\beta$ gliucum Koch. 52, 90 .

sexangulare L. $\mathbf{5 5}, 90$

Telephium L. $\beta$ purpureum L. 89.

var. $\delta$ et $\varepsilon \bar{i}$. 89.

villosum $\mathrm{L}, 15,41,90,168$. 


\section{XINII}

Selaginella spinulosa A. Br.41, 49, 14:

Selinum carvifolia L. $54,92$.

cerraria DC. 92.

Sempervivum Fanconneti Rent. 186.

Juratense Jord. 186.

Sene'cio alpestris Gaud. 98.

Doronienm L. 186.

flosenlosus Jord. Rent. ป̌1, 98.

Fuchsii Gmel. 5๊, 98.

Jacobrea L. 气ั4, รัย̆, 98.

var. flosenlosa Gaut. 98.

nemorensis L. 98.

Sarracenieus Gr. et G. 98

viseosus L. 57, 98.

vulgaris L. 54, 105.

Serapias latifolia L. 129.

longifolia L. 129.

Serratula arvensis L. 99.

nudicaulis DC. 15, $52,100,171$.

Vulpii Fisclı.-Ost. 187.

Seseli annuum L. 91.

bienne Crantz. 5ร, 91.

coloratum Ehrh.91.

Sesleria coerulea Ard. 50, 157.

Setaria glauea P. B. 157.

viridis $P . B .142$.

Sibbaldia procumbens L. 186.

Sideritis hyssopifolia L. 188.

Silaus pratensis Bess. 500, 95 .

Silene glareosá Jord. $18{ }^{\circ}$.

mitans L, 31, 71. 
Silene quadrifida L. 18\%.

Sinapis arvensis L. 54, 68.

var. Schkuhriana Reich. 54, 68.

Sisymbrium acutangulum DC. $1528,5 \% .66,15 \%$.

Anstriacum Jaeq. 66.

obtusangulum DC. 68 .

officinale Scop. 54,68 .

Sophia L. 54, 67, $15 \%$.

Thalianum Gand. 54, 68.

Solanum tuberosum $\mathrm{I}_{\text {. }}$ 50.

Soldanella alpina L. 187.

Solidago alpestris W. et K. 97

Sonchas arvensis L. 58, 101.

Sorbus Aria Crantz. 29, 88.

Aucuparia L. 29, 88.

Clamaemepilus Crantz. $\beta$ tomentosa Rent.

$$
186,191 .
$$

domestica L. $56,87$.

lıybrida L. 45̄, 88.

intermedia Reich. 88.

Scandica Fries. 29, $52,45,88$.

torminalis Crantz $29,88$.

Soyera montana Monn. 18:.

paludosa Godr. 48, 102.

Sparganinm erectum $\beta$ L. 127.

minimum Fries. $56,127$.

natans Gaud. non L. 127.

simplex Huds. 566. 127.

Specularia speculum A. DC. 105.

Spergula arvensis L. 58, 75.

saginoides L 72.

Spinacia inermis Moench. $ّ 0,120$.

spinosa Moench. 50, 120. 


\section{XLIX}

Spiraea Aruncus L. $50,80$.

filipendula L. 80.

Spiranthes aestivalis Rich. 44, 311, 150.

autumnalis Rich. 57, 150.

Stachis alpina L. 53, 116.

ambigua Sm. 192.

arvensis L. 51116.

recta $L .51,116$.

sylvatico palustris, 192.

Stellaria aquatica fl. fi. 72.

media, Sm. 73.

nemorum L, 42, 48, 72 .

uliginosa Murr. 48, 72.

Stipa Calamagrostis Gaud. 158.

pennata L. 48, 158

Streptopus amplexifolius DC. 188.

Swertia perennis L. 187.

'I'amarix Germanica L. Gaud. 89,

'Tamus communis L. 152.

'Taraxacum Dens-leonis Desf. 101.

laevigatum DC. 40, 101.

officinale Wig. var. alpinum Koch. 187.

palustre DC. 28, 101.

'T'axus baccata L. 24, 30, 125.

Tetragonolobus siliquosus Roth. 41, 41, 79.

'Teucrium chamaedrys L. 55, 117.

montanum L. 53 117.

Scorodonia L. 51, 117.

Thalictrum aquilegifolium L. $42,61$.

minus L. II saxatile Gaud. 45, 52, 61, 150 .

Thesium alpinum L. $18,121$. 
Thesium pratense Ehrh. 48, 121.

'Thlaspi arvense L. 54, 67.

Bursa-pastoris L. 68.

campestre L. 68.

Gaudinianum Jord. 185.

Leresehii Reut. 185.

perfoliatum L. 34, 68.

Thymus alpinus L. 115.

calamintha Auct. $11 \%$.

serpyllum L. $\mathbf{4 8}, \mathbf{1 1 5}$.

Tilia grandifolia Ehrl. 74

intermedia DC. Reut. 42, 52, 74.

mierophylla Vent. 42, 74.

parvifolia Ehrh. 74 .

platyphyllos Scop 59, 74.

sylvestris Desf. 74 .

Tormentilla erecta L. 82.

Tozzia alpina L. 187.

Tragopogon orientale L. $\beta$ aureus Rent. 187.

Trifolium agrarium Gaud. 78.

alpestre L. 57, 78.

arvense L. 58, 50, 79.

aureum Pol. 50, 78.

coespitosum Reyn. 195.

medium L. 48, 78 .

montanum L. 58. 78 .

ochrolencum L. 43 , 78.

pratense L. 58, 48, 79.

rubens L. 43, 78.

Triglochin paluktre L. $51,126$.

Trinia glaberrima Dub. 91.

Henningii Gaud. 91.

vulgaris DC. 44, 91. 
Triodia decumbens P. B. $45,140$.

Trisetum flavescens P. B. 18. 159.

'Triticum caninum Schr. 14.1.

moncoccum L. $38,142$.

nardus DC. Gaud. 14.

spelta L. 58, 142.

vulgare Vill. 58, 142.

Trollius Europaeus L. 26, 35, 40, 65.

Tulipa sylvestris L. 55, $\mathbf{1 5 2}$.

Turritis glabra L. 42, 46, 65, 154 .

Tussilago alpina L. 97.

farfarea L. 22, 97.

Petasites L. 97.

Renteriana Jord. 97.

Typha minima Hopp. 35, 126.

Ulmus montana Sm. 25, 30, 125.

Urtica dioica L. 50, 122.

urens L. $50,122$.

Utricularia minor L. $45,5 \% 1,117$.

vulgaris $L .51,117$.

Vaccinium myrtillus L. 55, 106.

uliginosum L. 187.

Vitis-Idaea L 35, 49, 106.

Valeriana angustifolia Tausch. 186.

dioica L. $28,96$.

montana L. $45,96$.

tripteris L. 45,96 .

Valerianella auricula DC. $38,96$.

Morisonii DC. $\propto$ leiocarpa God. 58, 96.

olitoria Moench. $58,96$. 
Veratrum album L. 188.

$\beta$ Lobelianum, 188.

Verbaseum floccoso thapsiforme Wirtg. 192.

lychnitidi-blattaria Koch. 192.

lychnitidi-floccosum Koch. 192.

nigro-floccosum Koch. 192.

nigro-lychnitis Schied. 192.

nigro-tlapsiforme Rap. 192.

nigro-thapsus Fries. 192.

nigrum L. $50,112$.

Schraderi Meyer, 112.

thapsiformi-blattaria G. et G. 192.

thapsiformi-floccosum Koch. 192

thapso-lychnitis M. et K. 192.

thapsus L. 55, 112

Veronica aphylla L. 187.

arvensis L. 54, 115.

Buxbaumii 'Ten. 25, 115.

didyma 'Ten. 54, 113.

filiformis DC. 115.

fruticulosa L. 42, 49, 115.

hederaefolia L. 54, 500, 115.

montana L. 42, 18, 115.

nummularioides Lec. et Lam. 187.

polita Fries. 115.

saxatilis Jacq. 187.

serpyllifolia L. 41, 115.

Teucrium L. 45, 115.

triphyllos L. 54, 115

urticaefolia L. 45, 115.

Viburnum Lantana L. 29, 94.

Opulus L. $29,94$.

Vicia cracea L. $52,79$. 
Vicia dumetorum L. 52, 79.

Faba L. 80.

sativa L. 55.80.

sepium L. 44, 79 .

sylvatica L. 48, 50, 70158 .

tenuifolia Roth. 59, 79.

Vinea minor L. 26, 109.

Vince-toxicum officiule Moench. 4k, 109.

Viola abortiva Jord. 20,70 .

agrestis Jord. 54, 71.

alba Bess. 20, 70.

alpestris Jord. 185.

arenaria DC. 18 .

biflora L. 185.

calearata I. 185.

canina L. 41, 70.

$\gamma$ lucorum Reich. 183

rar. minor Rent. 4!, 70.

var. nemoralis Rap. 70.

hirta L. 71.

mirabilis L. 25, $50,70$.

milticanlis Jord. 41, 70.

odorata L. 25, 71 .

var. albiflora, 20, 71.

palustris L 185

permixta Jord. 71.

pratensis Koch. 71.

pumila Ging. 70.

pumila Vill. 51, 70.

Riviniana Reich. 25, 70.

Sagoti Jord. 40, 70.

sciaphila Koch. 185.

scotophylla Jord. 21, 70. 
Viola segetalis Jord. $\mathbf{3 4}, \mathbf{7 0}$.

sylvatica Fries. 2\%, 70.

sylvestris Lam. 70.

$\beta$ Riviniana Koch. 70.

tricolor L. 70, 71 .

virescens Jord. 21, 70 .

Viseum album L. 2", 94 .

Vitis vinifera L. 41, 75 .

Vulpia ciliata Link. 5ே̈, 140.

myuros Reich. 140.

psendo-myuros Gay. 5.5, 140.

Zea Mays L. 142. 




\title{
PROIIRIDRS BOTHNIOIRS
}

\author{
AUX \\ VOIRONS
}

ET

\section{Supplément anx Ilerborisalions}

\section{A. S ALEVE}

\section{par Chs FAUCONNET}

Doct. en méd. des Facultés de Paris et de Genève, anc. méd. du Dispensaire, de l'Hôpital extéricur et du Bureau de Bienfaisance, anc. médecin en chef de l'Hôpital cantonal, Membre de la Soc. Helvétique des Sciences Naturelles, Membre honoraire de la Soc. Murithiemue de Botanique du Valais, elc.

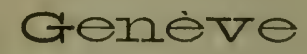

IMPRIMERIE GAREY FRÈRES, VIEUX-GOLLÉGE, 3 



\section{PROMUIIDES BOTLIIOLRS}

AUX

\section{VOIRONS}

FT

\section{Supplément aux Ilerborisations}

\section{A SALÈVE}

\section{par Ch PAUCONNET}

Doct. en méd. des Facultés de Paris et de Genève, anc. méd. du Vispensaire, de l'Hôpital extérieur et du Burean de Bienfaisance, anc. médrein en ché de l'Hôpital cantonal, Henlire de la Soc. Itrlvétique des Sciences Naturelles, Hembre honoraire de la Soc. Murithinenne de Botanigne du Valais, etc.

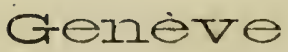

IMPRIERIE CAREY FRERES, VIEUX-COLLÉGE, 3 



\section{HERBORISATIONS.}

Voiri en que dit de Saussure de la végútation des Voirons dans le T. I de ses voyages, page $183, \S 977$.

"Les Voirons ne sont pas comme Salève fertiles en plantes rares; on n'y a trouvé que les plantes qui croissent dans les basses prairies et dans les basses forêts des Alpes, le Chrysosplenium alternifolium, la Cracaliu alpina, la Scandix odorata, te Thulictrum rquilegifolium, etc. Et une grand variété de Mousses, de Jungermania, de Lichens, de Champigmons, i'y ai cependant autrefois trouvé la Limnaea, qui n'est pas commune dans nos montagnes, mais je ne sais si on l'aura détruite en abatlant des forèts; all moins n'aije pas pu la retrouver."

Notre excellent Gandin consacre la courte notice suivante alıx Voirons: "Sabaudiæ Mons Lemano innminens; eum Arvae vallis occidentem versus a Salaeval dissociat. Rupes ejus stratis arenariis cum petra calcarea commixtis constant. Paulo infra culmen 4,259 p. s. m. elatum, olim in clivi orientalis tristissima solitudine? Monachii aliquot Benedictini vilam agebant; coenobio autem incendii flammis penitus consumpto, jampridem hancsedrm frigidam deseruerunt. Maximam montispartem ingentes occupant sylvae, ut nonnisi paucissimis locis 
illa splendida Alpium, tolius vicinac regionis pul chrique lacus scena compareat. Qno sane luculentissimo spectaculo, atque adeo pulchriori in proximo Salaeva multo commodius frui poteris.

Præler multas stirpes cryplogamicas sub ingentiun nomorum temmine lacte vigentes in eo monte occurrunt: Caculia alpina Jacq. (glabra Sul.), Chrysosplenium alternifolium, Limnaen borealis? (ibi olim a saussureo visam, serius Vir illustrissimus non amplius reperire potuil), Maluxis Loeselii Suv. (Ophrys L. ; and montis radices, in Arvite vallis paludibus), Myrrhis odorulu Scop. (Scandix L.), Neottia corduta Rich. (Ophris L.), Thalictrum aquilegifolium."

Co lableau est maigre et il a fallu les patientes recherches de notre savant ami Reuter et dont le résullat est consigné dans son Calalogue, pour avoir une idée un peu complète de la Flore des Voirons.

Dans les herborisations à Salève, j’ai signalé un cerlain nombre de plantes qui préfërent les terrains siliceux, savoir: Scleranthus perennis L.,S. biemnis Rent., Sedum villosum L. Arnica montana L., Pedicularis Inberosa L., Betula alba L., Alnus viridis DC., Deschumpsia flexuosa Griseb; Lycopodium clavatum L., Ceterach officinarum C. Bauh., et Pleris aquilina L. lies mèmes plantes se retrouvent aux Voirons sauf Sedum villosum, Amica montana et Pedicularis tuberosa; on devail s'y attendre en raison de la prédomiminance du terrain cristallin sur cette montagne. En revanche un certain nombre d'espèces dont la pluparl. affectionnent ce genre de terrain et qui manquent an Salève peuvent se récolter sur les Voirons. Ce sont: Silene rupestris L., Polygala depressa Wender., Geranium nodosum L., Trifolium spadiceum L., Geum rimel' L., Sedum annuum L., Galium rolundifolium L., Se- 


\title{
PROMENADES BOTANIQUES
}

\author{
AUX \\ VOIROMS
}

\section{AVERTISSEMENT}

Bien que les Voirons soient beaucoup moins riches, an point de vue holanique, que le Salèvo et le Jura, ils ne zont pourtant pas à dédaigner, et ce petit écrit fournira aux amateurs des indications précises sur les principales plantes qui sont l'ornement de celle monlagne, lout en faisant ressortir les différences qui existent entre la végétation des terrains où domine le gries et celle des terrains calcaires.

J'ai puisé mes renseignements dans mon herbier, dans les ouvrages de de Saussure, de Gaudin, de Reuter, de Riapin et dans le mémoire de M. l'Abbé Puget extrait du Tome $\mathrm{X}$ du Bulletin de la Société botanique de France. le dois également un bon nombre d'indications à mon respectable ami M. Le Curé Chavin et à il. Bernet qui s'occupe avec autant de rèle que dr succès de la botanique, de la géologie et des insectes de nos environs.

Ces quelques pages seront suivies d'un appendice qui servira de complément et de correction aux Herborisalions à Salève.

Genève, Mai 1868. 



\section{IN IIEMORIAM.}

C'élail pendant l'été de 1898, la roléc de belles-Leltres dont je faisais partic et qui comptait 22 éludiant: décida de fitire une promenade aux Voirons qu'elle n'avait pas encore visités. La plupart ayant répondu it l'appel, la bande jeune el joyeuse se mit en route par une belle soirée de Juillet et arpenta, avec toute la vigueur de jambes de 17 ans, la route de Jussy pour venir coucher à Si-Cergues. Après un souper quelconque, assaisonné par l'exercice et l'appétii, notre hôte nous enferma dans une espèce de grange en laissant à chacun le soin de se faire, à sa guise, un lit dans l. paille ou le foin, à tàtons el dans l'obscurité.

L'excitation de la marche ef les innombrables insectes qui peuplaient not'e couche assez dure ne nous perimirent guère de reposer', et il en résulta un mélange de plaintes, de rires et de chants qui nous valurent à Inaintes reprises la visile de notre hòte courıoucé auquel nous rendimes la tranquillité en parlant longtemps ivant le jour. Nous voulions voir lever le soleil, anssi l'ascension se fit-clle rapidement afin d'alteindre le sommet en temps opportun. Hélas! nous n'avions pas complé sur un épais brouillard accunnté autour de l'extrémilé septentrionale de la monlayne, alt-dessus 
des Ituines du couvent où nous étions arrivés: le sommet était alors convert d'une forêt de magnifiques satpins qui condensaient habituellement l'humidité de l'air du matin. Engourdis par le souffle froid d'un vent aigre el piquant, nous nous réfugiàmes dans les souterrains du couvent en nous empressant d'allumer du feu pour nous réchauffer; mais enfumés comme des blaireanx, il fallut promptement déguerpir au nilieu d'un concert de toux, d'éternuements el de récriminations. Ileureusement le brouillard malencontreux se: dissipa sous l'influence du soleil dont les rayons finirent par nous dégourdir, landis que nous admirions le superbe panorama au centre duquel nous élions placés. Lia promenade se continua le long de la crête qur: nous comptions suivre dans toute sôn étendue pour rédescendre sur Bonne; mais avec l'imprévoyance de la jeunesse nous n'avions guère songé aux provisions, d'ailleurs un orage nous menacait, en sorte qu'arrivés vers le milieu de la montiane nous descendimes comme une avalanche, en serrant nos ceintures pour calmer notre appélit qui fut enfin satisfait dans un des villages du pied.

Bien que 40 ans se soient écoulés, ceux qui vivent encore se souviennent loujours et reparlent souvent de de cette mémorable expédition. Mais hélas! combien nous ont déjà quiltés! Argand, Bourdillon, Crottet, Deonna, Prévost, Revilliod el Viridel ne vivent plus que dans nos souvenirs. Rappelés les premiers, il ont trouvé la solution du grand problème de l'existence; en touchant le port, ils sont entrés en possession de $c$ : bonheur atuquel nous aspirons lous et dont nous ne poursuivons ici-bas que l'ombre el l'apparence. Les survivants ne tarderont pas à les rejoindre, et la chainc: d'amitié brisée par le trépas se renoucra pour ne 
plus se dissoudre, car nous savons que c'est au sein de la mort que se trouve la vie.

On a dit qu'une parlie de l'existence se passe à désirer et à espérer, l'autreà se rappeler et à regretter; tourné depuis longtemps vers le passé, je songe souvent á ces amis dont la pensẻe fait revivre tant de bons momenls d'un temps qui n'est plus. C'est pourquoi je leur' consacre ces lignes, pieux souvenir d'une affection qui ne s'est jamais démentie el que n'a pu détruire leur départ prémaluré. Heureux quand la mémoire du cœur survit à li décadence des autres facultés. 



\section{INTRODUCTION.}

La physionomic riante, gracieuse et fiatche des Voirons contraste d'une fincon remarquable avec l'aspect rude, sec el sévère du Salève dont la lace occidentale s'élève perpendiculairement en formant un rempart en apparence inaccessible, landis que le flanc des Voirons qui regarde le Jura présente une succession de plateaux verdoyants, séparés par des pentes doucement inclinées.

Partoul des prairies luxuriantes, de gras pàlurages, des cultures variées, des groupes de maisons et dos chalels dispersés. Cà el là les resles des anciennes lorêts qui couvraient jadis la montagne et qui couronnent encore une partie des sommilés. Tout est plein de mysteres et d'altraits, aussi rien de plus délicieux qu'une promenade le long de la crête, en suivint les sentiers sinueux quila parcourent dans toute son élendue; loujours on marche sur un lin sablon ct presque conslamment on est à l'ombre d'une vécrélation aussi belle que variée. Quand se présente une éclaircie, on jouit d'une succession de lableaux splendides el variés, encadrés dans la verdure, formés par !e Buet avec son dòme de glace et le Mont-Blane avec les Aiguilles qui lui servent de corlége. S'élève-t-on sur les points culminants, on admire le magnifique panorama pré- 
senté par la chaine des Alpes à l'Orient, la ligne du dura i l'Ocrident, ct dans l'intervalle, par' les collines et les plaines qui encadiont la nappe bleme du Léman que siltonnent de nombreuses barques avec leurs blanches ailes et les batcaux ì vapenr avec leur panache de fumée.

Au pied de la monlagne du côté des Alpes, s'ouvre la vallée de Boëge dans laquelle on descend par des pentes rapides couvertes do magnifiques forêts de sapin.

Nalgré lous ses allraits la montagne des Voirons est moins visitée que le Salève. L'habilude, les souvenirs, la distance plus rapprochée, les nombreux moyens de communications, les bons hòtels de Nornex et de Nonetier atlirent la foule de nos compatriotes, an détriment des Voirons. Cependant depuis quelques années on a bàti sur le versant orienlal, au-dessous du Calvaire, deux hôtels-chalets offrant aux touristes le vivere et le couvert, el l'on a construil une route à voitures qui permet d'y arriver aisément, avec un détour considérable il esi vrai.

Quoique les Voirons soient i deux lieues de Genève, on peut cependant faire en un jour le tour complet de la montagne, pourvu qu'on parte en voilure et de bon matin. L'ascension peut se faire soit par la vallée de Boëge, soil par l'extrémité méridionale depuis le village de Bonne, soil par la Bergue el Lucinges, soil par St-Cergues, soit enfin par la Tour de Langin.

La crète est dirigée du Nord au Sud; elle présente deux sommets principaux, le Calvaire silué an-dessus des ruines de l'ancien couvent et qui s'élève à 1, 456 mètres au-dessus du niveau de la mer, et le Pralaire plus rapproche de l'extrémité méridionale et haut de 1,406 mètres seulement.

La composition géologique des Voirons diffëre beaucoup de celle du Salève; c'est gràces aux recherches 
des géologues modernes el en particulier anx éluder de MM. Vogt, de Mortillet el Favre qu'on est arrivé enfin i en comprendre la formation compliquée.

D'après la coupe donnée par ce savant géologne, it y aurait de bas en haut une suceession de couches do: molasse, de grès, de calcaire, le toul couronné par lo: grès nummulitique qui forme les rochers du sommet.

Voici du reste le résumé donné par M. Le Profinseur Favre :

1 Molasse de la base de la montagne.

2 Première zone de macigno alpin. ${ }^{1}$

3 Première zone (calcaire) néocomicnne, aux Ilivernages.

4 Seconde zone de macigno alpin.

5 Seconde couche néocomienne associes ì une couche à ammonite à sa partie extérieure, voisine de Chezllominal.

6 Terrain jurassique oxfordien de Chez Hominal.

7 Troisième couche néocomienne, au Fenil.

8 Troisième conche de macigno alpin avec grès it nummulites formant l'escarpement de la montagne.

Il existe en outre sur les Voirons des blocs erratiques abondanıs, au dessus de la Tour de Langins, ou ils s'élèvent jusqu'à 960 mètres environ. Dans le milieu de la montagne on en voit beaucoup aussi, ils sont asso ziés aux blocs erratiques crélacés de la valléc de la Drarice. Les argiles erraliques s'élèvent jusqu'a 1,000 mètres, et dans certains points les blocs alteignenl 1,046 mètres. Au bas des Voirons, du côté de Cranves el de Bonne, l'aceumulation du limon ghaciaire est considérable (Prof. Farre Vol. I $\$ 110$ ). Dans les en-

- Le macigno est un grès plus dur et plus ancien que la molasse. mais plus jeune et moins dur que le gress nummulitique du sommet. 
virons de Villards, vallée de Boëge, l'argile glaciaire remplic de cailloux polis et striés appartenant aux roches des monlagnes voisines, est lrès-épaisse (Prof. Favre $\$ 132){ }^{1}$.

J'ai parcour'u a plusieurs reprises la montagne des Voirons dans loutes les directions, cependant j'ai peu visité le versant qui donine la vallée de Boëge el dans les forèts duquel on pourrail pent-être trouver des plantes intéressantes. Si ces courses n'ont pas été tonjours bien fruclueuses au point de vue bolanique, elles ont toujours élé une source de plaisir et d'agrément, dont les épisodes se représentent souvent à la mémoire.

Il me souvient de deux étourdis, oublieux du poids des années, des leçons de l'expérience el de la prudence que devait leur inspirer leur litre de docteurs, Ifui eurent la fantaisie de s'engager dans un de ces chibles on couloirs qui servent i faire glisser le bois et qu'on reneontre entre la tour de Langin et le village de St-Ciergues. D'abord tout alla bien et la descente s'opéra avec rapidité; mais le couloir devint de plus en plus incliné et se transforma finalement en un ruisseau encitissé entre deux berges perpendiculaires. Un moinent ils eurent la bonne pensée de rebrousser chemin, malheureusement l'amour-propre en jeu, l'idée d'une rude grimpée à faire, l'espérance d'arriver bientòt en bas, leur inspirèrent la fatale résolution de continuer. "Quand le vin est tiré il faut le boire" dit l'un; malheureusemont it n'y avait rien at meltre en perce, rien que l'eau jaunatre du ruisseau, dans lequel ils pataugeaient. "Comme on fait son lit on se couche"

1 Voir pour de plus amples détails l'ouvrage remarquahle it lous ingards du I'rof. A. Favre, intitule. Recherehes géolongiques dans la partie de la Savoie, alu Piémont el de la Suisse voisine du. Mont-Bluner 1867. 
dit l'autre, en s'étendant tout de son long, à la suite d'une glissade sur les pierres roulantes du couloir. Enfin ils arrivèrent, mais dans quel élat! Ilarassés, rexténués, les mains en sang, les habits déchirés el couverts de boue, maugréant et protestant qu'on no les y reprendrait plus. Une halte au soleil pour se sécher, un calumet fumé avec délices et le fond de la gourde leur permirent de rejoindre à Moniaz le reste de la bande qui étail arrivée sans encombre en suivan! le chemin battu. Les jeunes ne raillèrent pas trop leurs anciens qui furent heureux de trouver une voiture dans laquelle ils purent dissimuler leur pileux équipage et rentrer en ville a l'abri des remarques malignes des passants. 
illa splendida Alpium, tolius vicinac regionis pul chrique lacus scena compareat. Quo sane luculentissimo spectaculo, atque adeo pulchriori in proximo Salaeva multo commodius frui poteris.

Præter multas stirpes cryplogamicas sub ingentium nemorum tegmine lacte vigentes in eo monte occurI'unt: Cacalia alpina Jacq. (glabra Sut.), Chrysosplenium alternifolium, Linnaeu borealis? (ibi olim a Saussureo visam, serius Vir illustrissimus non amplius reperire potnit), Maluxis Loeselii Suv. (Ophrys L. ; ad montis radices, in Arvate vallis paludibus), Myrrhis odorule Scop. (Scandix L.), Neottia corduta Rich. (Ophris L.), Thalictrum aquilegifolium."

co tableau est maigre et il a fallu les patientes recherches de notre savant ami Reuter et dont le résullat est consigné dans son Catalogue, pour avoir une idée un pen complète de la Flore des Voirons.

Dans les herborisations à Salève, j’ai signalé un certain nombre de plantes qui préferent les terrains siliceux, savoir: Scleranthus perennis L.,S. biennis Reut, Sedum villosum L. Arnica montana L., Pedicularis tuberosa L., Betula alba L., Alnus viridis DC., Deschampsia flexuosa Griseb; Lycopodium clavatum L., Ceterach officinarum C. Bauh., et Pteris aquilina L. Cies mèmes plantes se retrouvent aux Voirons sanf S'edum villosum, Arnisa montana et Pedicularis tuberosa ; on devail s'y attendre en raison de la prédomiminance du terrain cristallin sur cette montagne. En revanche un certain nombre d'espèces dont la plupart affectionnent ce genre de terrain et qui manquent au Salève peuvent se récolter sur les Voirons. Ce sont: Silene rupestris L., Polygala depressa Wender., Geranium nodosum L., Trifolium spadiceum L., Geum rivulr L., Sedum annuum L., Galium rolundifolium L., Se- 


\section{PROMENADES BOTANIQUES}

\section{AUX \\ VOIRONS}

\section{AVERTISSEMENT}

Bien que les Voirons soient beauconp moins riches, an point de vue bolanique, que le Saleve et lo dura, ils ne zont pourtant pas à dédaigner, et ce petit écriı fournira aux amateurs des indications précises sur les principales plantes qui sont l'ornement de cette monlagne, tout en faisant ressortir les différences qui exislent entre la végétation des ierrains où domine le grès et celle des terrains calcaires.

J'ai puisé mes renseignements dans mon herbier, dans les ouvrages de de Saussure, de Gaudin, de Reuter, de Riapin et dans le mémoire de M. l'Abbé Puget extrait du Tome X du Bulletin de la Sociélé botanique de France. de dois également un bon nombre d'indications à mon respectable ami M. le Curé Chavin el à il. Bernet qui s'occupe avec autant de zèle que dr succès de la botanique, de la géologie et des insectes de nos environs.

Ces quelques pages seront suivies d'un appendice qui servira de complément et de correction aux Herborisalions à Salève.

Genève, Mai 1868. 


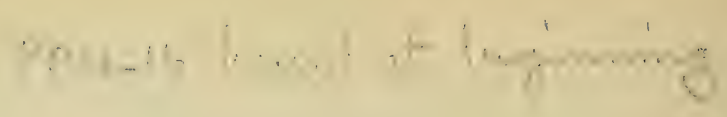


necio syluaticus L., Phylenma betonicaefolium L., PoIygonum bislorta L.. Eviophorum yracile Koch, Carex teretiuscula Good., C. limosa L., G. pilulifera L., Bromus secalinus L., Phegopteris alp stris Motl., Polystichum Oreopteris DC., Lycoportium annotinum L. et L. alpinum $\mathrm{L}$.

Je ne connais que quatre plantes qui soient spéciales anx Voirons ef qu'on ne relrouve pas dans les limites de notre Flore. Ce sont: Polygula depressa Wender. découvert par $11 \mathrm{H}$. Reuter ct Rapin en 1861 sur le sommel du còté de Boëge ct au-dessous du Couvent; Geranium nodosum L. découvert par II. Reuter an bord d'un pelit ruisseau on montant depuis Saint-Cergues à l'ancien Chalet Baumann par le sentier ordinaire (M. le Prof. Grenicr l'a indiqué par erreur dans notre Jura en confondant le Saint-Cergues de cette monlagne avec le village du mème nom au pied des Voirons. Il existe toritefois dans le Jura Français of M. Reuler l'indique au pi: d du llôle); Senccio sylraticus L., tronvé par 11. Reuter en 1861, a été récollé abondamment en 1867 par II. Pillard dans les bois de sapins près du sommel; Eriophorum grucile lioch n'a élí trousé jusqu'à présent qu'au marais de Lossy.

Les antres plantes rares et intéressantes des Voirons se rencontrent dans dautres localités de nos environs. Ainsi le Turritis glabu L. déconvert par .1. Bernet en 1861 dins un ravin an $2 / 3$ de la hauteur on montant depuis Bonne, existe à Prney, à Frontenex, près d'Arully, il Pommier et dans la rallée des L'sses; l'Arabis serpyllifolia L. trouvér parl M. Reuter sur les roches dures et compacles du grès nummulitique du sommet où il est en petile quantiti, si: trouve en abondance dans les funtes des rocher's calcaires du Brizon, du Vergy, du Jura at des Pitons du Salève; le Silene rupes- 
tris L. qu'on trouve sur la crête près des ruines du Couvent et du côté de Boëge, existe au mont Méry et dans la vallée du Reposoir, mais manque au Salève et au. Jura; le Trifolium spadiceum L., récolté par M. Reuter sur le second plateau au-dessus du Chalet nommé Châtillonet ne se retrouve qu'au Brizon et dans la Vallée du Reposoir; le Geum rivale L qui vit dans les pâturages, le long des ruisseaux, croît au Jura et au Brizon, mais manque au Salève. Le Comarum palustre L. qui existe au marais de Lossy, vient dans les tourbières du Jura et du Brizon; le Scleranthus perennis L. abondant an-dessus de Bonne, près du sommet méridional dans les couches sablonneuses, se retroure an Salère près de Cruseilles; il en est de même pour le $S$. biennis Reut. Le Sedum annum L. qui croît en petite quantilé sur les rochers près du Couvent, est commun au mont Vergy, au mont Méry et dans la vallée dı Reposoir, mais il ne se Irouve ni au Salève ni au Jura; le Ribes nigrum L. et le Myrrhis odorala Scop. qui existent dans les ruines du Couvent étaient autrefois cullivés par les Religieux, car on ne les retrouve nulle part ailteurs sur la montagne; le Chrysosplenium alternifolium L. qui croit sur les rochers près des ruines du Couvent, existe au Saléve, al Jura "i au Brizon; le Chaerophyllum cicutaria Vill. est dans tous les pàlurages du Jura, du Salève et du Brizon ; le Galium rotundifolium L. indiqué comme rare dans les bois de sapins du versant oriental par II. Reuter, a été découvert en abondanci par II. Bernet près de l'hòtel Chalel au-dessus de Boëge; c'est une des bonnes plantes des Voirons qui nt? se retrouve dans les limites de notre Flore que sur le Brizon; l'Adenostyles albifrons Reich. croit dans les. hois ombragés de nos autres montagnes; le Phyle:r- 
ma betonicaefolium Vill. trouvé par M. Reuter sur le revers du côté de Boëge au-dessus de l'hòtel Chalet n'est pas lare dans les pàturages de la vallée du Reposoir; le Rhododendrum ferrugineum L. dont il existe quelques pieds au-dessus du Couvent et dans la partie supérieure des bois du versant oriental, manque au Salève, mais habite les sommités du Jura, du Hôle, du Brizon, du Vergy el du Méry; le Polygonum bistorta foisonne dans les prairies du Jura et du Brizon; le Listera cordata Rich. inignonne Orchidée qui abondait autrefois dans la mousse sous les sapins, au-dessus du Couvent est'devenue Irès-rare depuis la destruction des forèls; en revanche elle prospère dans les bois frais et moussus du Jura, du Brizon et de la Vallée du Reposoir; le Corallorhiza innata R. Br. que j'avais recolté jadis dans la même localité a disparu, mais pourra peut-être se retrouver dans les bois du versant -oriental, il est rare dans les forêts du Jura, du Brizon et du Salève; le Liparis Loeseiii Rich. rare pour notre Flore, serait une spécialité du rnarais de Lossy oú il est abondant, si on ne lavait pas retrousé en petite quantilé au marais de Bossey sous Salève; il en est de mème du Sparganium minimum Fries qui existe an marais de Lossy et qui a été découvert par M. le curé Charin dans le marais de Viry oủ il esl plus commun; le Carex terelinscula Good. et le C. limosu L. existent daris lis marais tourbeux du Jura; He C. pilulifera L. late pour notre Flore et que l'on trouve dans les clairières dı sommel, n'a été signalé que dans une localitio du Vergy; le Deschampsia flexuosu Griseb. plante des sables siliceux caractérise les conthris sidérolitiques du Saleve: le Bromus seralinus L. quion trouve dins lis champs du pied de la monragne existe dans la plaine ol dans les champs du Bri- 
zon; le Botrychium lunaria Swartz est commun dans les pàturages de toules nos montagnes ; le Celerach officinarum C. Bauh. rare sur des vieux murs du village du Juvigny a élé signalé au Salève; le Polypodium vulgare L. est commun au pied de nos montagnes; le Phegopteris alpestris Metl. indiqué au-dessus du Couvent se retrouve au Jura, au Vergy, ct an Méry; le Polystichum Thelipleris Roth. rare au marais de Lossy se retroure à Rocllebol, à Troinex el à Divonne; le $P$. Oreopteris DC. qui vit sous les sapins près du Couvent, existe an Jura, dans la vallée du Reposoir, mais manque au Salève; le $P$. spinulosum DC. se retrouve dans les hois de sapins du Salève et du Jura; il en est de mème du P. Filix-Mas DC. du Cystopteris fragilis Bernh., de l'Asplenium Filix-foemina Bernh, de l'A. Halleri DG., de l'A viride. Huds. du Scolopendrium officinale Smith; et du Pteris aquilina L.; le Blechnum spicant Sw. indiqué sous les sapins audessus du Couvent, se relrouve au Salève, au Jura et au Brizon; le Lycoportium inundatum L. abondant au marais de Lossy croì également dans les tourbières du Jura; le $L$. annotinum $\mathrm{L}$. qui végète dans les bois de sapins au-dessus du Couvent peut se récolter dans le Ju'a et an Brizon, mais manque au Salève; le $L$. Selugo L. el le $L$ clavalum L. existent dans les bois de la partie supérieure de tontes nos montagnes; le $L$. alpinum signalé au-dessus du Couvent se retrouve au Brizon el manque au Salève; le Selerginella spimulosa A. Br. existe sur toutes tos pelouses des sommets de nos monlagnes; enfin l'Equiselum syluaticum L. se retrouve dans les bois de sapins du Jura, du Salève et du Brizon.

Le déboisement peu judicieux des Voirons a fail tort à certaines espèces, dont les uncs n'ont pas élé retrou- 
vées et les autres ont notablement diminué. Ainsi la Linnaea borealis L. et la Corallorhiza innata R. Br. ont disparu et devront être cherchées de nouveau dans les bois du versant oriental où $\mathbf{M}$. Bernet a retrouvé le Galium rotundifolium L. qui n'avait pas été revu depuis que II. Reuter l'avait signalé au-dessus de St-Cergues.

La Listera corduta Rich. qui étail abondante dans la mousse, au-dessus du Couvent, a presque disparu depuis qu'on a détruit les vieux sapins au pied desquels on la récoltail jadis; il en est de même de la Gymnadenia albida Rich. et de quelques autres espèces.

Ginq ou six promenades suffiront pour récolter la plupart des plantes intéressantes des Voirons; deux se feront au printemps, deux ou trois pendant l'été et la dernière en automne.

Pendant les mois d'Avril et de Mai on visitera les clairières des bois de Cranves, le marais de Lossy, les taillis du pied, et en dernier lieu la région moyenne de la monlagne.

On trouvera: Anemone nemorosa L., Corydalis tuberosa L., Viola alba Bess. avec ses variétés, $\boldsymbol{V}$. sylvatica Fries, V. Rivini ma Reich., Oxalis acelosella L. , Scorzunera humilis L. (en petite quantité dans les clairières des bois de Cranves), Primula grandiflora Lam., P. officinalis Jacq., P. variabilis Goup., Vinca minor L. , Pulmonaria tuberosa Schr. , Symphytum officinale L., Daphne mezereum L., D. laureola L., Salix aurita L., S. capraea L., S. repens L., Taxus baccala L., Pinus sylvestris L. , Orchis coriophora L. (à Cranves el à Lossy), O simia L., O galeala Lam. , 
O. purpurea Huds., Ophrys muscifera Huds., O. aranifera Sm., O. arachnites Reich., O. apifera Huds., (qui vivent en société dans les taillis près du marais de Lossy), Paris quadrifolia L., Scilla bifolia L., Schæ$n:$ s nigricans L., Eriophorum angustifolium Roth.,. $E$. gracile Koch (spécial au marais de Lossy), Carex dioica L., C. Davalliana Sm., C. pulicaris L., C. teretiuscula Good., C. paniculata L., C. leporina L., C. stellulata Good., C. remola L., C. vulgaris Fries, C. limosa L., C, ampullacea L. (tous à Cranves ou à Lossy), C. digitala L., C. ornilhopoda Willd., C. alba Scop., Anthoxanthum odoratum L. (dans les taillis), et Phleum asperum L. (entre Moniaz et S1Cergues).

Sur les murs du vieux château de Bonne se balancent de nombreuses touffes du Cheirmnthus Cheiri I.

Dans les bois du pied et de la région moyenne de la montagne croissent: Aquilegia vulgaris L., Actaea spicata L., Dentaria digitala L., D. pinnata Lam., Coronilla Emerus L., Sambucus racemosa L., Euphorbia dulcis L., Salix grandifolia Ser., Convallaria. maialis L., C. verticillata L., C. Polygonatum L.

Le Crocus vermis L. abonde dans tous les paturages, et l'on trouve en fleurs les Vaccinium Myrlyllus L. et V. Vilis-Idaea L.

Les Utricularia vulgaris L., U. minor L. et Menianthes trifoliata L., sont communes dans le marais de Lossy.

Les herhorisations de l'été el de l'automne ferone trouver un nombre plus considérable de plantes inté- 
ressantes, surtout sil'on parcourt avec soin les stations variées que présentent les Voirons.

Dans les localités humides du pied, telles que les clairières des bois de Cranves et surtout le marais de Lossy, on trouvera : Nymphaea aiba L., Drosera rolundifolia L., D. obovata L., D. longifolia L., Parnassia palustris L. , Comarum palustre L., Gratiola officinalis L., Pedicularis palustris L. , Menyanthes trifoliata L., Triglochin palustre L., Sparganium minimum Fries (rare), Epipactis palustris Crantz, Liparis Loeselii Rich., Gladiolus palustris Gaud. (abondant au milieu des clairières des bois de Cranves), Juncus obtusiflorus Ehrh., Rhynchospora alba Wahl., Scirpus panciflorus Light., S. compressus L., Glyceria fuitans R. Br., G. plicata Fries, Polystichum Thelipteris DC., et Lycopodium inundatum L.

Entre IIoniaz et St-Cergues, on récoltera: Scrophularia Balbisii Horn. et Mentha nepetoides Lej. (NI. aqualico-sylvestris $\mathrm{Ney}$.)

Dans les champs du pied et de la région moyenne se trouvent : Spergula arvensis L., Ervum Ervilla L., E. Lens L. (cult.), Lathyrus Gicera L., L. hirsutus L., Sonchus arvensis L., Phelipuea coerulea C. A. Mey. (près de l'ancien chalet Baumann, rare), Galeopsis praecox Jord., Stachys arvensis I.., Anchusa Italica Reutz. (près Bonne), Polygonum Talaricum L. (cult.), Bromus secalinus L. (au-dessus de St-Cergues), Lolium temulentum L. et toutes les autres plantes qui se rencontrent habituellement dans les cultures.

Le long des haies et parmi les laillis du pied, croissent: Astragalus Cicer L., Symphytum officinale L., Herminium monorchis R. Br., Luzula multifora Lej., Scirpus syluaticus L.. Carex arnithopoda Wild. C. leporina L., Phleum pratense L. $\beta$ intermeitium Gaud. 
Agrostis canina L. Triodia decumbens P. B., Gaudinia fragilis $\mathrm{P} . \mathrm{B}$; sur les vieux murs du village de Juvigny, végètent quelques louffes de Ceterach officinarum $\mathrm{C}$. Bauh.

Dans les bois de la montagne on trouve: Thalictrum aquilegifolium L., Ranunculus platanifolius L (vers le sommet), $R$. nemorosus DC., $R$. lanuginosus L., Aconitum lycoctonum L. , Cardamine syleatica L., Dentaria digitala L., D. pinnala Lam., Arabis Turrita L. (bois du pied), Turrilis glabra L. (au-dessus de Bonne, M. Bernet), Dianthus superbus L. , Moehringia trinervia Clairv., Stellaria nemorum L., Vio!a sylvatica Fries V. Riviniana Reich., Geraniumnodosum L. (rare) Spiraea Aruncus L., Vicia dumetorum L., V. sylvatica L, , Rubus saxatilis L. , R. cosius L., R. hirtus W. et N., R. Idaeus L., Rosa alpina L. avec ses variétés, $R$. alpestris Desegl. (d'après M. l'abbé Puget) R. spinulifolia Dem., R. vestita God. (près du Chalet Baumann) R. montana Chaix in Vill. (sur le plateau), $R$. mubrifolia Vill., R. mollissima Fries, R. subglobosa Sm., R. tomentosa Sm., R. Reuleri God., R. coriifolia Fries, $R$. platyphylla Rau. (II. l'abbé Pugei), $R$. dumetorum Thuill., Sorbus ancuparia L., S. Aria L., S. hybrida L. (au-dessus de Bonne), Epilobium spicalum Lam., Ribes alpinum L. Saxifraga rotundifolia L., Sanicula Europuea L., Sambucus racenosa L. Lonicera nigra L., L. Alpigena L., Galium rolundifolium L., (Bois de versant oriental, II. Bernet), Gr. sylcaticum L. . Asperula odoratu L., Knautia sylvatica Dub., Adenostyles albifrons Reich., Gnaphalium sylvaticum L., Soyera paludosa Godr. Vaccinium Myrtillus L., Rhododendrum ferrugineum L., Pyrola rotund ifolia L., P. minor L. (près du sommet), Pyrole secunda L. , Monotropa hypopylis L., Lysimachia nemo. 
rum L., Myosatis sylvatica Ehrh., Atropa Belladona L. , Digitalis grandiflora Lam., D. luteu L., Veronica urticaefolia L. V. montana L. (près du sommet,) Solıia ylutinosa L. Slachys alpini L., Fagus sylvatica L., Castanea vulgaris L. , Salix grandifolia Ser., S. capraea L., S.aurita L. Betulaalba L. (rare), Alnus viridis DC. (vers le sommet) Taxus baccata L., Juniperus communis L., Pinus sylvestris L., Abies excelsa DC. A. pectinata DC., Platanthera bifolia Rich. P. chlorantha Cust. Epipactis latifolia All., Cephalanthera pallens Rich., C. ensifulia Rich., C. rubra Rich., Neottia nidus-avis Rich. Listeru ovuta R. Br. L. cordala Rich. (rilre, all-dessus du Couvent), Corallorhiza imnata R. Br. (très-rare, se trouvait dans la mousse au-dessus du Couvent) Cypripedium Calieolus L. (bois du pied, rare), Convallaria verticillata L. , C. Polygonatum L., Maianthemum bifolium 1)G., Lilium Martagon L., Luzula Ravescens Gaud. L. maxima DC. (sommet), Carex pilulifera L. (rare, clairières du sommel), Calamagrostis montana DC., Milium effusum L. , Poa nemoralis L. Festuca sylvatica Vill., Elymus Europaeus L., Polypodium vulgare L., Phegopteris Dryopteris Fée, P. alpestris Melt. (au-dessus du Couvent), Aspidium aculeatum Doll. , Polysticum Oreopteris DG. P. spinulosum DG., P. Filix-Mas DG., Cystopteris fragilis Bernh., Asplenium FilixFoemina Bernh., A. Hulleri DC. , A viride II uds; Scolopendrium officinale Sil., Blechnum Spicant Sw. (audessus du Couvent), Pteris aquilina L. , Lycopodium Sclago L., L. annotinum L., L. clavatum L. (au-dessus du Couvent) L. alpinum L. (derrière le Couvent), Equisetum sylvaticum L.

Dans les prairies et les pâturages on récoltera : $R u$ nunculus aconitifolius L: (surtout dans les endroits 
humides), Trollius Europaeus L., Polygala depressa Wend. (sur le sommet du côté de Boëge et all-dessous. du Couvent, rare et spécial aux Voirons), $P$. vulgaris L., $P$. alpestris Reich. (pàturages du sommet), Viola canina L., V. alpestris Jord. (au-dessus de Bonne), Sagina Linnaei Presl. (endroils humides), Stellaria uliginosa Hurr. (mêmes localités), Hypericum quadrangulum Koch, Geranium sylvaticum L., 'Trifolium spadiceum L. (rare, sur le plateau), Geum rivale L. (prés humides), Potentilla aurea L., Alchemilla vulgaris L., Alchemilla hybrida Wild. (sommel), A. alpina L., Epilobium trigonum Schr., Astrantia major L., Carum Caryi L., Pimpinella magna L., Chaerophyllum cicutaria Vill., Myrrhis odorata L. (ruines du Couvent, probablement cultivé jadis par les Religieux), Homogyne alpina Cass., Antennaria dioica Gaertn., Soyera paludosa Godr. (prairies humides), Campanula rhomboidalis L., Phyleuma betonicaefolia Vill. (rare, au-dessus de l'Hòtel-Chalet), Vaccinium Vilis-Idaea L., Gentiana lutea L., G. verna L., G. ciliata L., Plantago alpina L. (sommet), Polygonum bistorta L. (prairies humides), Thesium alpinum L. (pâturages secs), Orchis globosa L., Gymnadenia albida Rich. (rare), Platanthera viridis Lindl., Nigritella angustifolia Rich., Crocus vernus L., Tofieldia palustris IIuds, Carex paniculata L., C. stelulata Good., C. vulgaris L. (localités marécageuses), Phleum alpinum L. (sommel), Sesleria coerulea Ari. (pàturages rocailleux), Deschampsia flexuosa Griseb. (prairies sablonneuses), Poa alpina L., Nardus stricta L., Botrychium Innaria L., Selaginella spinulosa R. $\mathrm{Br}$.

Sur les rochers el parmi les éboulis on trouve: Arabis alpina L., A, serpyllifolia Vill. (grès nummuliti-- 
que du sommet, rare), Silene mupestris L. (rochers du sommet du còté de Boëge, peu abondant), Moehringia muscosa L.. Scleranthus perennis L. (endroits sablonneux au-dessus de Bonne), S. biennis Reut. (mèmes localités), Sedum annuum L. (en petite quantité sur les rocher's près du Couvent), Chrysosplenium alternifolium L. (rochers humides du sommet), Saxifraga Aizoon L., Ribes nigrum L. (enceinte du Couvent, cultivé jadis par les Religieux), Valeriana tripteris L., $\boldsymbol{V}$. montana L. (éboulis calcaires), Senécio viscosus L., Campanula subramulosa Jord., Teucrium montanum. L. (éboulis calcaires), Rumex scutatus L. (Tour de: Langin). 


\section{NOTES}

Puisque j’ai parlé de la Linnaea borcalis, je vais dire tout ce quej'ai appris sur cette charmante petile fleur.

Le genre Linnaea a été dédié au célèbre botaniste suédois par Gronovius, probablement par correspondance ou veıbalement, car je n'ai trouvé aucune description due à cet auteur ${ }^{1}$.

Linné, dans sa Philosophia botanica (Ed. II, p. 156, 1783) accepte la paternité du genre Limnaea qu'il a fait suirre de la leltre $G$, pour indiquer que Gronovius en a élé le parrain.

La planle est décrite dans la Flore Laponaise, dans la Flore de Suède et dans le Species plantarum; il en existe une assez bonne figure à la fin de la Flora Suecica.

D'après Linné elle habite les vieilles forêts moussues du Nord (Laponie, Suède, Norvége, Sibérie, Russie, Canada) et de la Suisse. Ledebour lui assigne également pour domicile les forêts touffues de l'Altaï.

En Allemagne, on la trouve en Prusse, en Poméranie, dans le Ilolstein, le Nlecklembuurg, la Silésie, la Bohème et le Tyrol. Elle a été indiquée en Piémont par Reichenbach, mais Allioni ne la mentionne pas.

On la retrouve en Grande-Brelagne, surlout en Ecosnica.

2 J -F. Gronovius, médecin belge, a publić en 1739 une Flora virgi- 
se - où je l'ai récoltée, - et dans une seule localité d'Angleterre, dans le Northumberland: elle manque en Irlande.

En France on l'avail indiquée dans les Cévennes, à Monipellier, dans les Vosges el l'Alsace; mais déjả de Candolle, dans le dernier volume de la Flore française, affirme qu'elle ne se troure ni aux Cévennes, ni aux Voirons; Duby l'indique comme douteuse pour la France; enfin Grenier et Godron l'exclvent complétement.

Nos auleurs suisses: Haller, Gaudin, Suter, Murith, Hegelschweyler et Moritzi l'indiquent dans les forèts de sapins et de mélèzes de la chaìne méridionale de nos Alpes; ils signalent plusieurs localités des Grisons et du Valais; Suter mentionne le St-Gothard, et la plupart citent les Voirons d'après de Saussure.

Le chanoine Murith, dans sa lettre du 27 Juin 1794 à Abraham Thomas, s'exprime ainsi : "En continuant à suivre les bords du torrent (le Trient), j'eus le plaisir de cueillir dans un bois la Linnaea borealis qu'on n'avait trouvée, jusqu'ici, que dans lesGrisons et à Saas ». Abraham Thomas dans sa lettre du 15 Juillet 1795 au chanoine Murith luidit: "Dans cette gorge (vallée de Saas) au delà du pont, sur la gauche de la Viége, est une petite croix dalée de 1733 , avec celte marque 1. P. C'est là que la belle Linnuea borealis croît en quantité. »

Enfin d'après le Prodrome, elle habite les forèts moussues de la Laponie, de la Suède, de la Norvége, de la Russie, de l'Allemagne, de la Suisse, de la Savoie, de l'Ecosse, de la Sibérie el de l'Amérique du Nord (Canada, Nouvelle-Angleterre, Vermont, NewHampshire, Terre-Neuve).

La Suisse serait donc la station la plus méridionale de celte espèce. 
Celle petile plante, aussi modeste que gracieuse, se plaît à l'ombre des forêts de sapins, de mélèzes, de pins et d'aroles; eile cache dans la mousse ses souches ligneuses, sarmenteuses, grêles, allongées, rampantes et rameuses, supportant des tiges dressées, garnies de feuilles pétiolées, opposées, petites, arrondies, légèrement dentelées et velues; ces tiges sont terminées par des fleurs géminées en forme de clochettes suspendues, d'un blanc rosé et d'une odeur douce et pénétrante, qui trahit leur présence, surtout le soir. Le calice est à cinq folioles entourées à la base d'un petit involucre à 4 parties; la corolle, en forme de cloche régulière, est à 5 lobes ; les élamines sont au nombre de 4 dont 2 plus courtes; à la fleur succède une baie sèche, à 3 loges contenant chacune 2 graines. La plante est toujours verte.

On l'emploie dans le Nord en infusion, comme un remède spécifique dans les douleurs goulteuses et rhumatismales ; les habitants font également des calaplasmes avec la feuille.

Je me rappelle encore la joie avec laquelle je cueillis cette jolie fleur au pied du Ben Lawers, an-dessus de Loch-Tay, en Écosse, arec le professeur Graham, qui enseignait alors la botanique à l'université d'Edimbouror et qui conduisait ses éléves dans une herborisasion aux Highlands.

Depuis lors, je ne l'ai rerue vivante qu'en Valais, ou M. Ph. Privat, qui en avait fait une ample provision dans la vallée de Tourtemagne, ent la bonlé de m'en donner quelques beaux échantillons tout frais, il y a près de deux ans. 
Polygala depressa Wend.

Syn. P serpyllifolia Weihe. P. vulgaris depressa Fries P. déprimé.

Cette espèce décrite par Wenderoth a étẻ trourée aux -Voirons par MIM. Rapin et Reuter en 1860. Elle n'avait été signalée qu'aux tourbières des Rousses par M. Garnier d'après Godet. Suivant Thurmann, c'est une plante des terrains argileux et qui évite les zones calcaires.

Elle habite en Allemagne les prairies tourbeuses de $I_{a}$ Souabe, el de la contrée du Rhin jusqu'en Westphalie et en Holstein.

En France elle est indiquée en Lorraine, dans les Vosges, en Alsace, en Auvergne, aux environs de Paris, en Normandie, à Nantes, dans le Lyonnais et aux Pyrénées. En Angleterre elle parait fréquente sur les Bruyères du North-Yorkshire (F. G. Baker).

En résumé, c'est une plante rare mais qui se trouvera probablement dans d'autres localités.

Geranium nodosum L. Geranium noueux.

Linné l'indique seulement en Dauphiné. En Suisse il est rare, et n'a été vu que dans le canton du 'Tessin et sur le versant sud du Dessemberg (Jura) oủ M. Shutlleworth l'a Irouvé en abondance.

Pour les environs de Genève, il n'existe qu'aux Voirons au-dessus de Saint-Cerģues oú il a élé découvert par M. Reuter et an pied du Môle.

Il habite l'Allemagne da Sud, le Tyrol, le littoral de 'Adriatique, le Piémont, le Nilanais, etc.

On le trouve pour la France dans les bois monta- 
gneux du Jura, du Dauphiné, de la Provence, des Cévennes, des Pyrénées, de la Région centrale (Sources de la Loire, Mont Pilate, Monts-Dores, Monts-Dômes, etc.)

Il ne figure pas dans le Catalogue des plantes de l'Algérie de N. Nunby.

En Angleterre il est indiqué dans les monlagnes du Cumberland et dans deux localités dı North-Yorkshire (J.-G. Baker).

C'est une des plantes rares de nos environs et du petit nombre de celles qui sont spéciales aux Voirons.

Senecio sylvaticus L. Seneçon des hois.

Cette plante, rare en Suisse, habite les forêts sablonneuses; elle est indiquée dans les bois du canton l'Appenzel; aul-dessus de Fouly en Valais; près de Paycrne, de Bursins, de Gimel et dans le Jorat au canton de Vaud; aux gorges du Seyon et dans d'autres localités du Jura Neuchàtelois et Françair: aux environs de Bàle, de Porentruy et en Argovie. Pour notre Flore locale il n'a élé signalé qu'aux Voirons oú il a été decouvert par M. Reuter en 1861 el retrouvé abondamment par M. Pillard en 1867 dans le bois près du sommel.

Le Prodrome lui assigne pour habitation les forêts sablonneuses de presque loule l'Europe (Allemagne, Piémont, Saroie, France el Angleterre).

II. Boissier n'en fait pas mention dans son voyage en Espagne.

C'est une des plantes spéciales aux Voirons. 
Liparis Loeselii. Rich.

Syn. Ophrys Loeselii L. , 0. Iiliifolia Lam. Malaxis Loeselii Sw. M. uliginosa Clairv, Sturmia Loeselii Reich.

Liparis de Loesel.

Cette jolie Orchidée se troure en abondance au marais de Lossy dans les touffes de Sphagnum, et il en existe de rares échantillons au marais de Bossey au pied du Salève : c'est presque une spécialité des Voirons.

Bien que Gaudin la signale comme fort rare en Suisse, elle existe néanmoins dans plusieurs localités marécageuses des cantons de Zurich, de Bàle, de Soleure, de Lucerne, de Berne, du Valais et de Vaud. Linné l'indique en Suède ot en Russie; elle se trouve dans presque toute l'Allemagne, en Piẻmont, en Angleterre et en France; cependant elle parait manquer dans la Còted’Or, les Bouches-du-Rhône, le Gard, les Pỹrénées, la Charente, l'Ouest et l'Algérie.

Gladiolus palustris Gaud.

Syn. : G. Boucheanus Schlechtend. G. neglectus Schl. Glayenl des marais.

Celte espèce décrite par Schlechtendal sous le nom de G. Bortheumus en la dédiant à M. Bouché, a recu de Gaudin le nom de G. palustris qui a été adoplé par presque lous les autenrs. M. Nicklès (notice sur les lilayeuls de France et d'Aliemagne) en donne une bonne figure.

Ce glayeul n'est pas commun en Suisse, nous le possédons aux environs de Genève dans les bois de Cranves au pied des Voirons oú il est commun, aux marais de Pouilly près de Saint-Genix, dans les environs 
de Chalex el dans les bois de Peissy. Gaudin l'indique dans la Suisse transalpine, dans le Voralberg, aux environs de Noville, de Vouvry et de Roche. Pour l'Allemagne on le trouve en Prusse, en Bohême, en Autriche, en Istrie et en Tyrol. Pour la France il n'est indiqué qu'en Alsace, surlout aux environs de Benfeld, et près de Nanlua. Il parail manquer en Angleterre et il n'est mentionné ni dans la Flore d'Allioni, ni dans le voyage en Espagrae de II. Boissier. C'est une des plantes rares de la Flore des Voirons.

\section{Eriophorum gracile Koch.}

Syn.: E. triquetrum Hopp. E. polygsachium rar. $\gamma$ L. Linaigrelte grêle.

Cette expèce généralement rare n'à élé trouvée qu'au marais de Lossy oú elle est indiquée par MM. Reuler, Rapin et Godet. Elle existe dans quelques localités marécagenses de Bàle, d'Argovie, de Zurich, de Berne et de Vaud; dans les marais spongieux de certaines parties de l'Allemagne; elle ne paraît pas commune en France et en Angleterre. C'est une des bonnes plantes de nos environs.

\section{Carex pilulifera L.}

(Syn. C. decumbens Ehrh. C. filiformis Pollich.) Laiche pilulifère.

Ce carex n'est pas très-commun en Suisse; pour nos environs il n'a été trouvé que dans les c'airières des bois près du sommet des Voirons el all mont Verogy. 
Il a été signalé dans le canton de Berne, le Bas-Talais, le Tessin, la vallée du Rhin, le Jorat et le Jura Neuchâtelois et Vaudois, soit sur les pelouses sèches et élerées (Godet), soit au marais de la Vraconnaz (Rapin).

Les auteurs français l'indiquent dans les bois de presque toute la Frince: il existe de même dans les forêts de l'Allemagne et en Angleterre, Lindley, Hooker et J. G. Baker le regardent corrme fréquent.

En tout cas, c'est une des rares plantes des Voirons el de notre Flore locale. 



\section{SUPPLÉMENT}

AUX

HERBORISATIONS A SALÈVE

c 



\section{ADDITIONS AU CATALOGUE}

P. 62. Ranunculus Auricomus L. Renoncule dorée, Bouton d'or. Se trouve dans les taillis entre Presilly et Pommier. Avril, Nai. (.I. l'abbé Puget.)

P. 65. Barbarea Augustana Boiss. (B. intermedia Bor.) Barbarée d'Aoste. Extrémité méridionale du Salève, le long de la route près de Cruseilles; sur les Bornes entre La Roche et le Plot; sur les montagnes entre Thorens et le petit Bornand. Avril, Mai. (II. l'abbé Puget:)

1d. B. striclu Andrz. (B. vulgaris auct. rec.) B. roide. Commune dans tout le bassin de La Roche. (II. l'abbé Puget.)

P. 67. Sysimbrium |Sophia L. croît dans le cimetière d'Arenthon. (II. l'abbé Puget.)

P. 70. Viola mirabilis L. Bois près de Reignier. (M. l'abbé Puget.)

P. 71. Diunthus superbus L. Eillet superbe. Audessous des Pitons, à la lisière des bois entre la Traversière et le chalet de la Tuile; au bois d'Yvres du còté de La Mure toût, Septembre. (M. Didier.)

P. 72. Holosteum umbellatum L. Holostés en ombelle. Bords des haies et des champs aux environs de Reignier. Nars, Arril. (Il. l'abbé Puget.) 
P. 75. Geranium palustre L. Bois de Reignier. (II. l'abbé Puget.) Retrouvé par M. Reuter entre le Châble et Pommier.

P. 78. Anthyllis vulneraria L. Anthyllide vulnéraire. Conslitue en quelque sorte le fond de la végétation des pâturages et des prés secs du Salève. Juin, Juillet.

P. 88. Epilobium rosmarinifolium Haenk. (E. Dodonaci Gaud.) Epilobe à feuilles de Romarin. Croît dans les débris calcaires entre Veyrier et Crevin. (I. Rapin.) Au mont Gosse. (M. Bernet.) Juillet, Août, Septembre.

Г. 93. Hedera Helix L. ne fleurit qu'en automne.

P. 96. Valeriana tripteris L. surtout au-dessus d'Archamp.

P. 97. Petusites albus Gaertn. (Tussilago alba L.) Petasite blanc. Cà et là dans la Combe, au-dessous de la station de l'Atragene et sur les escarpements entre la localité de la Serratula et le sentier de la Traversière. Mai, Juin. (II. Rapin.)

P. 107. Erica camea L. Bruyère incarnate. Près du chalet des Avenières, au-dessus de Cruseilles, sur les couches sidérolitiques; découverte par M. A. Metford. Avril, Mai.

P. 108. Cyclamen hederaefolium Ait. (C. Neapolitanum T'en.). Ne se tronve pas dans le vallon des Usses, ainsi que je l'ai indiqué par erreur, mais croit parmi des buissons rocailleux exposés au milli, au-dessous des roches qui dominent le chemin d'Allonzier à Choisy, a $2 \%$ kilom. de l'église d'Allonzicr. (Note de M. l'abbé Chevallier). Ce Cyclamen est identique a celui qui est au-dessus de Roche, canton de Vaud. 
P. 112. Digitalis grandiflora Lam. n'est pas dans les buissons du pied de Salève, mais dans les taillis au sommet du chemin de la Croiselte. (II. Reuter.)

P. 113. Veronica Buxbutmii Ten. croît à SaintBlaise. (M. l'abbé Puget.) Il est commun aux environs de Baden, en Argovie, dans les cultures et les prés.

P. 131. Iris Germanica L. Iris d'Allemagne. Se trouve spontané près de Reignier d'après M. l'abbé Pugel. Nlai.

P. 132. Erythronium dens-canis L. croît aussi dans le ravin du Viézon. (II. Reuter.)

P. 140. Festuca tenuifolia Sibt. Se trouve aussi sur le Petit-Salève et surtout sur les pàturages du sommet à terrain sidérolitique. (M. Reuter.)

P. 143. Ceterach officinarum C. Bauh. Retrouvé en 1867, par M. le ministre David, sur le vieux mur qui soutient la Croix entre Monnetier et Mornex, par l'ancienne route, où il l'avait déjà vu en 1837. 


\section{ADDITIONS AUX NOTES}

L'Atragene alpina L. a été trouvée au Mont-Gargan, au-dessns de Moutiers en Savoic (II. l'abbé Pug̣et).

Helleborus viridis L. Notre plante se distingue par ses fleurs grandes et peu nombreuses (une ou deux a chaque tige); elle n'a aucun rapport avec l'Helleborus Bocconi Ten. (II. multifidus Vivian. Fl. Dalm.) dont j'ai vu des échantillons dans l'Herbier Boissier; elle diffère également de l' $H$. viridis de l'Onest de la France et du Nord de l'Espagne dont les fleurs sont plus petites et plus nombreuses, les feuilles plus étroites et plus forlement dentelées. M. Reuter a donné aux échantillons espagnols de son herbier le nom de $H$. Occidentalis. Notre plante est bien celle décrite par Gaudin, Koch, Reichenbach, etc.

Le mont Salève est riche en Arabis: ces plantes qui se plaisent dans les éboulis calcaires existent en abondance an pied de la montagne, surtout dans les endroits qui ont été récemment remués. Dans une seule herborisation on pourra facilement récolter vers la fin de Ilai et au mois de Juin les espèces suivantes:

\section{Arabis alpina L. Arabette des Alpes.}

Elle est commune sur les rochers, parmi les éboulements, jusqu'auprès du sommet du Salève, du Jura, 
des Voirons, du Mòle, du Brizon, du Vergey et du Méry. C'est une des espèces les plus répandues et que l'on trouve presque sur toutes les montagnes de la Suisse, de l'Allemagne et de la France.

\section{Arabis auriculata Lam.}

Syn. : A. recta Vill., A. patula Wallr., Turritis palula Ehrh. Arabette à oreillettes.

Cette plante décrite par Lamark sous le nom d'Arabelle oreillée, Arabis auriculata, lui avait élé envoyée par \$. Liollard sous le noun de Planta nova Fabii columnae; il l'indique en Dauphiné dans les lieux pierreux. Gaudin la mentionne comme assez fréquente parmi les rochers el sur les murs des endroits chauds de la Suisse, en Valais et au Salève au-dessous du Pasde-l'Echelle.

Les antres auteurs suisses ajoutent le Fort de l'Ecluse et les environs de Salins. Elle habite l'Espagne, l'Europe centrale, le midi de la France, l'Alsace, l'Allemagne méridionale, la Hongrrie, la Transylvanie, la Bohême, l'Autriche, le Piémont, le Royaume de Naples et la Sicile.

Elle n'est pas commune dans nos environs et ne se Irouve qu'au pied du Salève, au Pas-de-l'Echelle, audessus de Crevin et aux rochers du Coin; elle est plus abondante autour du Fort de l'Ecluse. Je l'ai récoltée près de Moutiers en Tarentaise.

Elle manque aux Voirons.

\section{Arabis saxatilis. All.}

Syn. : A. nova Vill. Arabelte des rochers.

Gaudin l'indique comme rare sur les rochers des Alpes, d'Aigle et du Valais. 
Les autres auteurs suisses mentionnent en outre les rochers du Salève, les éboulements autour du Fort de l'Ecluse et le Jura soleurois.

Celle espèce, peu commune dans nos environs, a été trourée en petile quantité aux rochers dı Coin; elle est plus abondante autour du Fort de l'Ecluse. Elle existe dans la vallée du Reposoir et au-dessus du Petit-Bornand. Elle manque aux Voirons. Je l'ai récoltée en Valais près de Martigny au pied du mont Ottan.

En France on la trouve sur les montagnes calcaires du Dauphiné et des Pyrénées, mais elle paraît manquer en Allemagne et en Angteterre. Nos montagnes seraicnt donc la station la plus septentrionale de cette espèce qui, dans le Prodrome, n'est mentionnée spécialement qu'en Savoie et en Dauphiné.

\section{$4^{0}$ Arabis hirsuta Scop. Koch. \\ Syn. : Turritis hirsuta L. A. hérissée.}

Cetle espèce qui croit dans les prés secs ou tourbeux du pied de nos monlagnes, dans la plaine et sur les murs, se reconnaît à ses grandes tiges effilées, hérissées de poils surtout à la base; les feuilles radicales sont en forme de spalule et rétrécies en petiole, celles de la tige sont embrassantes à oreilleltes tronquées; la grappe des fleurs s'allonge après la floraison, les siliques sont dressées, linéaires, comprimées et présentent sur toute la longueur de chaque valve une nervure saillante; les graines sont comprimées, ovales, finement ponctuées, el très étroitement marginées au sommet (Reuter, Catal. ed. 2). Elle doit se trouver un peu partout dans des localités analogues. 


\section{$5^{\circ}$ Arabis incana Roth, Reich.}

Syn.: A. conferta. Wild. Reich. icon. A. blanchàtre.

Cette espèce abondante au pied dı Salève et du Jura, autour des carrières et dans les rocailles, se disting̨ue de la précédente par ses tiges moins effilées, plus nombreuses, plus hérissées de poils simples et rameux; la grappe est moins allongée et les fleurs sont ramassées en corymbe pendant l'anthèse; les siliques sont comprimées, sub-tetragones, toruleuses, à nervures dorsales saillantes. (Reuter catal. éd. 2.) Cette plante se reconnaît Irès-bien par son port el sa physionomie qui permeltent de la distinguer à distance de ses congénères. Je ne l'ai pas vue au pied des Voirons.

\section{$6^{\circ}$ Arabis sagittata Bertol. DC.}

Reich. icon. A sagillée.

On peut facilement confondre celle espèce avec les deux précédentes et ce n'est qu'en les étudiant souvent sur le vif, aux diverses périodes de leur développement. et dans leurs slations, que l'on arrive à les différencier.

Elle a un aspect plus vigoureux, les leuilles sont profondément dentées, plus sagittées, avec les oreillettes appliquées sur la tige; les fleurs sont déprimées en corymbe pendant l'anthèse ; les siliques sont plus longues, plus étroiles, comprimées, toruleuses, leur nervure médianc disparait au-dessus du milieu; les semences sont comprimées, oblongues, rétuses aux deux extrémilés, étroitement marginées et finement ponctuées. (Reuter catal. éd. 2.)

On la trouve le long des haies et des chemins au pied du Salève et dans la plaine. Elle est moins commune 
que les autres et je ne crois pas l'avoir rue au pied des Voirons.

$7^{0}$ Arabis alpestris Schleich. Reich. Icon.

Syn. : A. hirsuta I sessifolia Gaud. A ciliata $\beta$ hirsuta Koch A arcuata Shntl. $\beta$ hirsuta God. (Reut. catal. ed. 2.) A. alpestre.

Celte espèce bien caractérisée habite les pâturages rocailleux des parties élevées du Saléve, du Jura, du Môle el du Brizon; je ne l'ai pas vue aux Voirons, mais peut-être la Irouverait-on sur les conches calcaires de la montagne. Elle croît sur les montagnes du Dauphiné et aux Pyrénées. Nous n'arons pas en Suisse l'Arabis ciliatu $\dot{\mathrm{R}}$. Br. qui habite les bords de la mer en Irlande el en Ecosse.

\section{$8^{\circ}$ Arabis muralis Bertol. A. des murs.}

Celle espece commune parmi les rocailles du pied du Salève et du Jura, vit sur les vieux murs de Villenenve, de Vevey al darns quelques antres localités du Valais. On la tronse it l'Est el au Midi de lit France el en Italie. Elle parait manquer en Allemagne el en Angleterre.

\section{Arabis hybrida Reut. A. hybrite.}

Celte forme intermédiaire à l'A stricla Iluds. el à l'A. muralis Bert. a été décrite et figurée par II. Reuter dans le supplément à la lre édition de son calloggue. Jusqu'à présent elle n'a été trourée qu'autour des carrières du pied du Salève entre le Pas de l'Echolle et Grevin. Peut-ètre la décolivrirat-on au Fort de l'Ecluse ou les parents se trouvent en société. 


\section{$10^{\circ}$ Arabis stricta Huds.}

Sjn.: A. hirta Lam. A multicaulis de Sauss., Turritis Rayi Vill., All; Sutter. Arabette roide (Godet) A. de Ray (Gaudin).

Gaudin lui donne pour habilation les rochersexposés au soleil, des localités chaudes du sud-Cucst de la Suisse; il la signale comme fort rare.

On ne la trouve pas dans les limiles politiques de notre pays, et tous les autres auteurs suisses ne mentionnent que les rorher's du Salève et ceux du Jura, au Fort de l'Ficluse et au-dessus de Thoiry.

Elle manque en Allemagne et dans le Nord, existe en Piémont, au Mont-Cenis, au Mont-Yesoul, et près de Hussey (Allioni).

Villars qui l'admet sous le nom de Turritis Raii en donne une figure assez bonne et signale plusieurs localités du Dauphiné. II fait observer que Rai ct Haller ont très-bien vu etdécrit celte plante qui a été confondue avec les Turritis, Ies Arabis, la Cardamine petraea et le Sysymbriuın Barrelieri L.

Les divers auteurs français ne la signalent que dans le Dauphiné, à la Fontaine de Vaucluse et dans les Pyrénées. M. Boissier ne la mentionne pas daus son roỵage en Espagne.

En Angleterre elle n'est indiquée que su' les rochers de St-Vincent près de Bristol (Lindley, Hooker).

Si l'on en excepte la localité anglaise, le mont Salève serait la station la plus septentrionale de cefle espèce rare et peu répandue. 


\section{$11^{\circ}$ Arabis serpyllifolia. Vill.}

Arabelte à feuilles de serpolet.

Gaudin lui assigne comme habilation les rochers des Alpes et des montagnes de la Suisse occidentale, dans le canton de Vaud et dans le Valais.

Elle se tronve en Savoie et en Piémont; pour la France on sigrale le Jura, les Alpes du Dauphiné, de la Provence et les Pyrénées.

Celte espèce que nous avons aux Pitons du Salève, sur le rocher de la Dòle, au sommel du Brizon et sur les rochers du Vergy, existe en petite quantité dans les fentes des rocher's de grès nummulitique du sommet des Voirons. Elle n'est jamais abondante dans ses diverses stations. Elle manque en Allemagne el en Angleterre.

\section{$12^{\circ}$ Arabis turrita. L. A. Tourelle.}

Cette espèce, la plus grande du genre, est commune dans les buissons du pied du Salève et se trouve dans quelques localités de la plaine. En Suisse, on la trouve dans le canton de Bàle, dans le Jura, au Valais, au canton de Vaud, au Tessin et dans les Grisons. Elle habite le Midi de l'Allemagne, un grand nombre de localités de la France, surto:ıt l'Est et le Midi. On la trouve aussi en Angleterre.

Le Turritis glabra, L., a élé trouvé au-dessus des bains de Salins, près de: Moutiers en Tarentaise, par I. l'abbé Pugel.

Le Sisymbrium aculangulum, DG., est commun sur les rochers à la base de la monlagne de Veyrier, près Annecy; il se trouve près de Faverges, au mont Thobert et au pied du mont Charvin (II. l'abbé Puget). 
L'Arenaria grandiflora, L., se trouve sur les montagnes qui s'étendent de Boëge à la Dranse; spécialement sur le mont Ilermance, au-dessus d'Habère-Lullin, et sur le mont Laout, au-dessus de Vailly (II. l'abbé Puget).

Anthyllis montana, L. (Syn.: Vulneraria montana scop. Lam.) Anthyllide des montagnes.

Celte jolie espèce, très-rare pour la Suisse, n'est indiquée par Gaudin qu'au Salève et à la Dôle. On la trouve néanmoins dans d'autres localilés de la chaîne neuchàteloise et française ; elle habite l'Allemagne méridionale (Autriche, Croalie, Tyrol, Trieste), le Piémont, la Savoie, la France (Jura, Còte-d'Or, Dauphiné, Provence, Cévennes, Pyrénćes orientales et centrales). II. Boissier ne la mentionne pas dans son voyage en Espagne, et elle manque en Angleterre. C'est une des bonnes et rares espèces de nos environs.

L'Ononis rolundifolia, L., r's! commune dans les environs de Mouliers et de St-Jean-de-Maurienne (M. l'abbé Puget). Je l'ai récoltée au bois Champion, près de Brides en Tarentaise, et près des hains de Louëche en allant aux Echelles.

L'Exonymus latifolius, Scop., est abondant sur les bords de l'Arly, entre Ugine et. Flumet; il a été tronvé par N. Songeon aux environs de Chambéry et sur le Crêt du Maure, au-dessus d'Annecy (II. l'abbé Puget).

Je ne possède pas d'échantiilons de ces diverses localités, et il serait intéressant de les comparer avec la var. $\beta$ de Gaudin de l'Evonymus Europaeus ou avec la var. $\beta$ macrophyllus de Schleicher.

La Vicia syleutica, L., est commune dans la vallée de Boëge el sur la montağne de Veyrier, près Annecy (N. l'abbé Pugel). 
La Polentillu rupestris, L., existe au vallon des Usses, au pied des rocher's, en descendant aux bains de la Caille du côté de Cruseilles et an bois Champion, près de Brides la Perrière (II. l'abbé Puget).

La Potentilla petiolulata, Gaud., se trouve aussi sur le Roc-de-Chère au bord du lac d'Annecy, entre Menthon cet Talloires (M. l'abbé Puget).

Rosa vestita, God., se trouve sur la colline d'Allonsier, près des bains de la Caille, au mont Nivolet, près de Chambéry, et à Saint-Romain, Isère (M. l'abbé Puget).

Rosa Salaevensis, Rap., a été récolté au mont Nivolet et à Bellevaus, Haute-Savoie, pa: M. l'abbé Puget.

Rosa spinulifolia, Dematr.

Syn.: R. spinulifolia \& Dematratiana Thory. R. rubiginosa var. spinulifolia Ser. in D. C. prodr. Rosier i feuilles spinulifères.

Gaudin indique ume seule localité du canton de Fribourg, au-dessus de Châtel-Saint-Denis, où le doyen Dematra le découvrit et donna le premier sa description dans une monographie des rosier's du canton de Fribourg.

Les autres auteurs suisses l'ont retrouvé au Wassernüh (en Argovie), sur le Jura et au Salève.

Seringe dans le Prodrome ne cite que Fribour et Nerviers.

Ce beau rosier cultivé à Paris, an Jardin de Lixembourg, s'est maintenu sans changements au milieu d'une foule d'autres ispèces el de variétés.

Nous le trouvons puur les environs de Genère, an Salève, anx Voirons, an Jura, en montant suit à la Dôle, soit au Reculet. 
Il n'a pas encore élé signalé en Allemagne; Koch l'indique à Obergesteln, en Valais, d'après Shuttelworth.

Greniel et Godron n'rn font pas: mention dans leur Flore de France, il parail manquer en Angleterre.

Rosa montana Chaix in Vill. est considéré par M. Godet, qui l'a éludié dans le Jura Neuchâtelois, comme une véritable expèce et non comme un hybride. $M$. l'abbé Puget qui l'a v'r près Aime en Tarentaise, ne croit pas que ce soit l'espèce de Gap; cependant M. Reuter a reçu de Gap et de l'Isère des échantillons identiques avec notre espèce.

Le Scleranthus perennis L. a été récolté par M. l'abbé Puget sur la colline des Allinges, près de Thonon.

Le Hieracium lanatum L. est assez répandu au pied des montagnes qui s'étendent d'Annecy-le-Vieux à Ugines: il se trouve à Moutier et à Chambéry. (M. l'abbé Puget).

Le Hieracium Andryaloides Vill. existe au mont Thobert, près de Faverges, el près de Chambery ( $M$. l'abbé Puget).

Erica carnea L. Jacq.

Syn. E. herbacea L., L saxatilis L. Bruyère incarnale.

Celte élégante bruyère dont les nombreuses touffes de fleurs d'un rose vif s'épanouissent au premicr printemps, a été découverte par M. Netford an-dessus de Cruseilles, près du Chalet des Avenières, e'est une plante i joindre á la liste de celles qui caractérisent les couches sidérolitiques du Salève.

Elle existe en outre an pied du Vòle sous les rochers d'Andey et à Dessy près de Bonneville.

Les auteurs suisses l'indiquent dans diverses localités des montagnes de Zurich, de Berne, de Lucerne, de 
Glaris, des Grisons, de Vaud et du Valais (elle est abondante au Bois-Noir entre Saint-Maurice et Evionnaz).

On la trouve en Autriche, en Bohême et en Silésie. Allioni l'indique en Piémont, au-dessus de Tende et en Savoie.

Elle paraît manquer en France et en Angleterre.

Cependant le Prodrome admet une variété $\beta$. Occidentalis qui se trouverait dans les montagnes de l'Irlande, les environs de Bordeaux, le Portugal et la région méditerranéenne.

Le Daphne alpina L. existe dans les rocailles de la montagne de Veyrier près d'Annecy (M. l'abbé Puget).

P. S. Pendant que ces pages élaient sous presse, j'ai eu le chagrin de perdre un digne et bon ami don: le nom revient souvent dans ces notes. Je reproduis ici un article bien court et bien incomplet qui a part dans le Journal de Genève du 30 Mai.

La paroisse de Compesières vient de perdre le respectable curé qui la desservait depuis près de trente ans, M. l'abbé Chavin, enlevé, à l'âge de 69 ans, par une maladie inattendue, à ses nombreux amis.

Zélé, actif et bon, il était aimé de tous ses paroissiens; pour les pauvres il avait toujours quelque secours en réserve; pour les malades et les affligés de douces consolations; pour tous de sages conseils et d'utiles directions.

Il avait été longtemps précepteur dans le canton de Fribourg, et c'est en donnant ses leçons qu'il avait pris le goût de la botanique qu'il a cullivée avec amour jusqu'à son dernier jour; c'est à cette époque qu'il se jia d'une étroite amilié avec 1. le docteur Lagger et 
avec M. D. Rapin, auteur de la Flore du bassin du Léman.

L'excellent Gaudin a consigné fidèlement dans sa Flore helvétique les nombreuses communications que lui envoyail l'abbé Chavin.

Plus tard il se lia également avec MM. J. Muret, Leresche, Reuter, directeur de notre Jardin des plantes, l'abbé Chevalier, du séminaire d'Annery, M. le docteur Dupin, M. Edm. Boissier el beaucoup d'autres botanistes auxquels il communiquait libéralement les nombreuses plantes qu'il récoltait lui-même ou qu'il recevait de ses correspondants.

Unis par les mêmes goûts pendant près de trente ans, nous n'avons eu que des relations agréables, sans que le moindre nuage les ait jamais troublées. D'accord sui les principes essentiels, nous éritions les questions secondaires qui n'auraient amené que des discussions inutiles.

Ce que j’aimais en lui, c'était l'homme intérieur, aussi instruit qu'aimable et bienveillant. Savant et modeste, il avait une conversation sérieuse et enjouée; ses yeux bleus brillaient et sa bonne figure s'animait dès qu'il était question d'une course pour chercher quelque plante rare, ou nouvelle pour nos environs; nous étions alors comme des écoliers en vacances, jouissant en plein de ces excursions pendant lesquelles il se livrait à toute l'expansion de son caractère.

Franc et loyal, il détestait tout ce qui n'était pas droit, surtout quand il était question de botanique.

Son hospitalité élait simple et cordiale, aussi ses amis étaient sûrs d'être reçus avec joie lor'squ'ils allaient frapper à la porte de l'ancienne Commanderie qu'il habitait et qui porte sur son fronton l'inscription sui- 


\section{4}

vante: Hanc aedem struxit melioris amor. Il nous la traduisait ainsi : Le désir d'une meilleure demeure a présidé ì la construction de cel édifice. Malheureuscment pour nous, il n'a que trop tôt trouvé celte meilleure demeure qu'il espérait.

Sadex-sous-Prangins, 28 mai 1868.

Ch. F. d.-m. 


\section{Lisle des principaux Ourrages consullés}

C. Linnaei Genera plantar. ed. 2. Lugd. Batav.1742.

C. Linnaei Flora suecica. Lugduni Batavorum 1745.

C. Linnaei Philosophia botanica Viennae 1763 et 1783 .

C. Linnaei Species plantar. ed. 3. Vindobon, 1764 .

Linné-Willdenow Sp. plantar. Berlin 1797-1810.

G. Gmelin. Flora badensis alsatica. Carlsrhue 1805.

G. F. A. Ledehour. Flora altaica. Berlin 1829-1833.

L. Reichenbach. Flora germanica excursoria. Leipsic 1830-1833.

Fred. Wimmer. Flora von Schlesien. Berlin 1832 et Breslau 1340.

C. S. Kunth. Flora berolinensis. Berlin 1838.

J. C. Dòll. Rheinische, flora. Francfort 1843.

W. D. J. Koch. Synopsis Florae germanicæ et helveticæ. Ed. 2. Francfort S/II. 1843.

W. D. J. Koch. Synops. der deutschen und schweizer Flora. Ed. 2. Leipsic 1846.

Dr K. J. Kreutner. Taschenbuch der Flora Wiens. Vienne 1864.

J. A. Scopoli. Flora carniolica. Vienne 1760.

G. Allioni Flora pedemontana. Turin 1785.

J. E. Smith. Compendium Flora britannicæ, Ed 5. London 1828. 
J. Lindley. Synopsis of the british Flora. Ed. 2. London 1835.

Sir W. Hooker. The brilish Flora. Ed. 5. London 1842. J. Dickinson. The Flore of Liverpool. London 1851.

J. G. Baker. North York-Shire. London 1863.

C P. Hobkirk. Notes on Crataegus. Hudder'sfield 1866.

A. Gouan. Flora monspeliaca. Lyon 1765.

Villars. IIistoire des plantes du Dauphiné. Grenoble 1786-1789.

de Lamarck. Encyclopédie méthodique. Botanique 1789. Lamarck. Flore francaise. Paris, an III de la Rép. de Lamarck et de Candolle. Synopsis plantarum in Flora gallica descriptarum. Paris 1806. de Lamarck el de Candolle. Flore française. Paris 1815. Picot de la Peyrouse. Histoire abrégée des plantes des Pyrénées el supplément, Toulouse 1818.

De Candolle. Prodromus. Paris 1824-1866.

G. Bentham. Catalogue des plantes des Pyrénées, etc. Paris 1826.

Loiseleur-Deslongchamps. Flora gallica. Paris 1828. J. E. Duby. Bolanicon gallicum ed. 2. Paris 1828.

A. Mutel. Flore du Dauphiné. Grenoble 1830.

Lorey et Duret. Flore de la Côte-d'Or. Dijon 1831.

C. Pauquy. Flore du dép. de la Somme. Amiens 1831.

A, Mutel. Flore française. Paris 1834-1837.

Kirscheleger. Prodr. de la Flore d'Alsace. Strasb. 1836.

F. Chevallier. Flore générale des environs de Paris.

Ed. 2. Paris 1836.

Noulet. Flore du bassin sous pyrénéen. Toulouse 1837. Cosson el Germain. Flore des environs de Paris. Paris 1845.

A: Jordan. Mémoires 1846-1857.

Phil. Babey. Flore jurassienne. Paris 1846.

Grenier el Godron. Flore de France. Paris 1848-1855. 
A. Boreau. Flore du centre de la France. Ed. 2. Paris 1849.

Seringe Flore des jardins. Lyon 1849.

Desmoulins. Erythraea et Cyclamen. Bordeaux 1851.

Hanry. Partie botanique du Prodrome d'histoire naturelle du dép. du Var. Draguignan 1853.

G. Lloyd. Flore de l'Ouest. Nantes 1854.

E. Nichalet. Notice sur quelques plantes du dép. du Jura et du pays de Gex. Besançon 1854.

C. Contejéan. Enumération des plantes des environs de Montbeillard. Besançon 1854.

Lemaoult et Decaisne. Flore des jardins et des champs Paris 1855.

De Pouzolz: Flore du Gard. Nimes 1856-1862.

Graves. Calalogue des plantes du dép. de l'Oise. Beauveais 1857.

Songeon et Perrier. Notes sur des plantes rares ou nouvelles de la Savoie. 2 fascicules 1859.

Cosson et Germain. Synopsis analytique de la Flore des environs de Paris. Paris 1859.

Philippe. Flore des Pyrénées. Bagnères 1859.

C. Desmoulins. Catalogue des phanérogames de la Dordogne. Bordeaux 1859.

A. Tremeau de Rochebrune et A. Savatier. Catalogue des plantes de la Charente. Paris 1860.

V. Payot. Catalogue des Fougères, Prêles et Lycopodiacées des environs du Mont-Blanc. Paris 1860.

L'abbé Cariot. Etude des Fleurs. Lyon 1860.

A. Chatin. Excursion botanique en Savoie et en Suisse.

Paris 1861.

Castagne et Derbès. Catalogue des plantes des Bouches du Rhône. Marseille 1862.

L'abbé Puget. Mémoires dans les bulletins de la Société botanique de France 1863-1866. 
Gillet et Magne. Nouvelle Flore française. Paris 1863. Blanche et Malbranche. Catalogue des plantes de la Seine-Inférieure. Rouen 1864.

Ch. Grenier. Flore de la chaîne jurassique. Paris 1865. G. Munby. CataI. plantarum in Algeria, etc. Ed. ? London 1866.

Dr Bouvier. La chaîne des Aravis 1866.

Edm. Boissier. Voyage botanique dans le midi del'Espagne en 1837. Paris 1839-1845.

Ch. Martin. Du Spitzberg au Sahıra. Paris 1866. 


\section{AUTEURS SUISSES}

Pour les anciens auteurs qui ont écrit sur les plantes suisses, il importe de consulter le grand ouvrage de A. von Haller: Historia stirpium indigenarum Helvetiae, Berne 1768. Préface, p. X et suivantes ainsi que les Icones Plantarum Helvetiae, édition publiée à Berne en 1813 par J. S. Wyttenbach.

Dr Vicat. Histoire des plantes vénéneuses de la Suisse. Yverdon 1776.

Von Sternberg, Reise in die Rhetischen Alpen. Nürnberg 1806.

J. C. Schleicher. Cal. plantarum Helvetiae. Bex 1807.

Le Chanoine Murith. Guide dı Botaniste en Valais. Lausanne 1810.

Gaudin. Agrostologia helvetica. Genève 1811.

Manuel d'herbor. en Suisse et en Valais. Zurich 1811.

Villars, Lauth et Nestler. Précis d'un voyage botanique en Suisse. Paris 1812.

G. Wahlenberg de vegetatione in Helvelia. Zurich 1813.

N. C Seringe. Essai sur les Saules de la Suisse. Berne 1815.

Hagenbach. Tentamen florae Basilaeensis, avecle su pplément. Bâle 1821-1834-1843. 
Suter et Hegetschweiler. Flora Helvetica. Ed. 2. Zurich 1822.

J. G. Krauer. Prodromus Floræ Lucernensis. Lucerne 1824.

Gaudin. Flora Helvetica. Zurich 1828.

$\mathrm{D}^{\mathrm{r}} \mathrm{J}$. Hegetschweiler. Versuch uber helvetischen Arten von Rubus. Zurich 1829.

G. F. Reuter. Calalogue. Genève 1852.

A. Moriizi. Die Pflanzen der Schweiz. Chur 1852.

H. Lebert. De Gentianis in Helvetia nascentibus. Zurich Mars 1834.

Gaudin et Monard. Synopsis. Zurich 1836.

R. Blanchet. Cat. des plantes du canton de Vaud. Vevey 183.3.

Em. Thomas. Catal. des plantes suisses. Lausanne 1837. Th. Wegelin. Fnumeratio stirpium Florae helveticae, Zurich 1838.

A. Morilzi. Die Pflanzen Graubundens. Genf 1838.

A. Koelliker. Phanerogamische Gewachse des canton Zurich. Zurich 1839.

J. Hegretschweiler et 0. Heer. Flora der Schweiz. Zurich 1840.

Carl Nageli. Die Cirsien der Schweiz. Neuchàtel 1840. Reuter. Supplément au Catalogue. Genève 1841.

D. Rapin. Guide du botaniste. Lausanne 1842.

J. P. Brown. Catalogue des plantes qui croissent aux environs de Thoune. Thun 1843.

Moritzi. Die Flora der Schweiz. Zurich 1844.

Méthode analytique. Payerne 1846.

J. Wartmann. St-Gallische Flora. St-Gall 1847.

R. Blanchet. Les champignons comestibles de la Suisse. Lausanne 1847.

J. Thurmann. Enumération des plantes vasculaires du district de Porrentruy. 1848. 
J. Thurmann. Phytostatique du Jura. Berne $1 \S 49$.

C. Godet. Flore du Jura. Neuchàtel 1852.

Dr E. Cornaz. Enumération des lichens jurassiques. Neuchâtel 1852.

Bulletins de la Société Hallérienne. Genève 1852-1856. Contejean. Sur la dispersion des plantes à Montbéliard. Porrentruy 1853.

Botanistes jurassiens. Bulletins des nouvelles stations de plantes du Jura. Porrentruy 1855.

D. L. Fischer. Taschenbuch der Flora von Bern. Berne 1855.

Dr Bernouilli. Die Gefallkryptogamen der Schweiz. Basel 1857.

H. Christ. Pflanzengeographische notizen uber Wallis. Basel, 23 Décembre 1857.

Dr Steiger von Buron. Flora des canion Luzern 1860. Reuter. Gatalogue. Ed. 2. Genève. 1861.

Dr Mercier. Rubi genevenses. 1861.

Favre-Guillermot. Champignons comestibles du canton de Neuchâtel 1861 .

D. Rapin. Guide dı botaniste. Ed. 2 Genève 1862.

H. Christ. Uhersicht der europäischen Abietinen. Basel 1863.

D'Angreville. Flore valaisanne. Genève 1863.

Ch. Godel. Plantes vénéneuses du cant. de Neuchâtel. 1864.

Thomas a Bruhin. Flora Einsielensis. Einsiedlen 186 亿. Schobinger-Pfister. Taschenbuch fur reisende botaniker in canton Luzern. 1866.

$\mathrm{D}^{\mathrm{r}} \mathrm{H}$. Christ. Uher die Verbreitung die Pflanzen etc. Basel 1866.

Actes de la Société helvélique des sciences nalurelles. De Saussure. Voyages dans les Alpes. Genève 1796-1804. 
A. Favre. Considérations géologiques sur le mont Salève. Genève 1813.

A. Favre. Recherches géologiques dans la partie de la Savoie, du Piémont et de la Suisse, voisine du Mont-Blanc. 1867. 


\section{TABLE DES MATIÈRES}

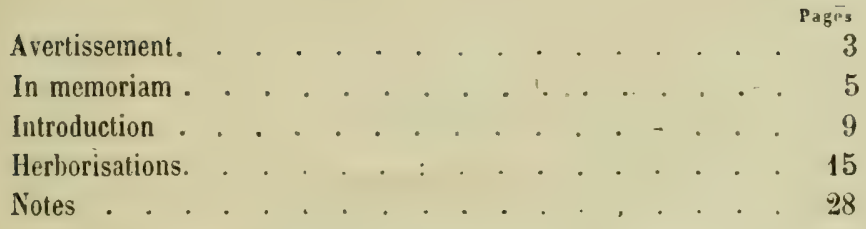

SUPPLÉYENT AUX HERBORISATIONS A SALÊVE

Additions au Catalogne . . . . . . . . . . . . . 39

Additions aux Notes . . . . . . . . . . . . . 42

Notice sur l'abbé l'havin . . . . . . . . . . . 52

Liste des Ourrages consultés . . . . . . . . . . . . 59

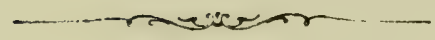







\title{
EXCURSIONS BOTANIOUES
}

\author{
DANS \\ LE BAS-VALAIS \\ par le Dr GH. FAUGONNET
}

Äncien médecin eu chef de l'Hônital de frenàve

Membre de la Société helvétique des Sciences naturellos de la Socićté murithienne de botanique

du Valais, etc.

\section{GENEVE \& BALE}

H. GEORG, LIBRAIRE-EDITEUR

MAI 1872 



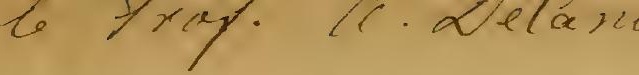
Herninege yespatie EXCURSIONS BOTANIQUES

JANS

\section{LE BAS-VALAIS}

par le Dr CiH. FAUGONNET

Aucien médecin en chef de l'Hôpital de Genève Memhre rle la Société helvétique des Sciences naturelles de la Société murithienne de botanique du Valais, etc.

\section{GENĖVE \& BALE}

H. GE 0 H, LI B RAIRE-ÉDITEUR

\section{MAI 1872}



DÉDIÉ

A LA SOCIÉTÉ MURITHIENNE

de Botanique du Valais 



\section{INTRODUCTION.}

Le Canton du Valais est un des plus riches de la Suisse en plantes rares et intéressantes. Non seulement on y rencontre presque toutes les espèces alpines qui se plaisent près des g̨laciers, dans les vallées supérieures ol sur les sommets élevés, beaucoup d'espèces méridionales qui affectionnent les localités les plus chaudes de la plaine; mais encore il fournit aux botanistes un contingent respectable de plantes qui ne se trouven pas ailleurs dans notre pays ou qui du moins y sont fort rares.

Celte circonstance a développé le goût de la botanique dans ce Canton, chez quelques médecins, et surtout chez les Rev. chanoines du St-Bernard el du Simplon. Linné n'était point resté étranger à l'étude des plantes du Valais el avail donnè le nom de Silene Valesia à une espèce nouvelle qui lui avait été communiquée. Le celèbre Haller avail fait des voyages botaniques secondé dans ses recherches par Abraham Thomas le premier de la dynastie qui en est aujourd'hui à sa qua. trième génération de bolanistes. Suter, dans la première édition de sa Flora helvetica, fait une mention spéciale des plantes du Valais. Mais le premier ouvrage spécial est celui du Rev. Prieur Nuritl qui publia, en 1810, un ouvrage précieux dans lequel il résume le résultat de 
ses nombreuses herborisations et de celles d'Abraham et de Louis Thomas. Il donne d'abord leur correspondance - car c'est par lellres qu'ils se communiquaient mutuellement le résultat de leurs explorations - la pre. mière est datée du 14 février 1793 et la dernière du 3 août 1806. Il consacre la seconde partie à un calalogue alphabétique des différentes plantes dont ils avaient constaté la présence en Valais, en indiquant avec soin les localités el l'époque de la floraison. C'est toujours avec un vrai plaisir qu'on relit ces leltres écrites avec simplicité et naturel sans autre prétention que l'exactitude et la vérité.

L'auteur de l'entomologie helvélique, de Clairvil:e, fit pataitre en 1811 un Manuel d'herborisation en Suisse et en Valais avec de nombreux délails sur les plantes de ce pays. Gandin dans sa Flore suisse donne une large place aux plantes du Valais el dans le $7 \mathrm{me}$ volume (Topographia botanica), il signale les espèces intéressantes que l'on peut trouver dans les différentes localités de ce pays.

Mentionons l'excellent ouvrage de Koch (Flora Germanica et Helvetica), la Flora Germanica excursoria de Reichenbach, la seconde édilion du Guide du botaniste dans le Canton de Vaud, par D. Rapin qni décril toutes les espèces qui croissent dans le Bas-Valais, enfin les ouvrages généraux de IIegretschweyler (Flora der Schweiz, Moritzi (Flora der Schweiz.), A. Gremli (Excursions Flora für die Schweiz, 1867 et supplément 1870). J. G. Ducommun (Taschenbuch für die Schweizerischen Botaniker 1869) et J. Rhiner (Tabellarische Flora der Schweizer Kanlone, 1869 avec supplément). Les linera alpina de J. Scheuchzer publics en 1723 et le précis d'un voyage botanique en Suisse et en Valais par Villars, Lauth et Nestler, donnent lindication d'un 
bon nombre d'espèces appartenant à la contrée dont nous nous occupons.

Depuis Murith, il y a eu peu de publications concernant spécialement la Flore du Valais; je ne connais qu'un Mémoire du Dr II. Christ de Bàle intitulé : Pflanzen geographische Notizen über Wallis, un ourrage de feu d'Angreville sous le titre de: Flore valaisanne, mais qui n'est qu'une simple énumération d'après l'ordre naturel des familles ${ }^{1}$; le bulletin des Iravaux de la Société Murihienne pour les années 1861 à 1867, ainsi que le Guide du botaniste su: le Grand St Bernard par feu le chanoine Tissière. o vvrage édité par la susdite Socicté dont il fut l'un des fondateurs et qu'il présida jusqu’à sa mort.

Citons encore les catalogues de Schleicher el de Thomas qui avaient fait de nombreuses herborisations dont les résultats ont prouvé que le Valais contient une très-forte proportion ces plantes qui constituent l'ensemble de la Flore suisse.

Enfin les herbiers de Messieurs Nuret, Leresche, Rapin, Reuter eic., renferment toutes les espèces signalées en Valais.

Pour terminer, ajoutons que feu le chanoine Rion a laissé un manuscrit qui n'est qu'un catalogue méthodique des plantes de son pays. Cet ouvrage verra le jour, cit-on, prochainement; mais il sera nécessairement incomplet, car l'auteur, mort depuis plusieurs années, n'avail pas encore mis la dernière main à son travail qu'il ne complait publier que plus tard.

${ }^{1}$ Il est à regretter que l'auteur n'ait pas communiqué son manuscri! ¿̀ quclque membre compétent de la Sociẻté murithienne dont il avait été l'un des fondateurs; il aurait évilí les nombreuses erreurs qui déparent son ouvrage et qui ont soulevé de sévères critiques à la suite desquelles il dut retirer son volume de la circulation. 
Espérons qu'il paraitra enfin une bonne Flore descriptive du Valais, pays, qui, avec les Grisons et le Tessin, possède la plus riche collection de ces plantes rares que le botaniste aime à récolter, et qui font la gloire de la Flore suisse.

Ayant parcouru, à plusieurs reprises, la vallée du Rhône depuis le Bouveret jusqu'à Sierre, avec MII. Muret, Rapin, Reuter, Mercier, Chavin et Dupin, j'avais résumé sous forme de lettres nos différentes courses pour faire connaitre les espèces les plus intéressantes dont nous avions enrichi nos herbiers. Ces lettres ont paru en partie dans le Journal de Genève en février el en avril 1869; je les ai complétées en ajoutant le résultat d'herborisations faites en été et à la fin de l'automne. De plus, j’ai dressé une liste de:s plantes que l'on trouve dans les différentes localités que nous avons visilées el j’ai rédịé des notes sur les espèces rares, ainsi que sur les plantes spéciales au Bas-Valais. Ces indications dorneront, j'espère quelque facilité aux botanistes qui auraient l'intention de faire des excursions dans le Bas-Valais. 


\section{Excursions botanigues.}

Genève, 10 février 1869.

\section{Cher ami,}

Afriandé par la douceur exceptionnelle de cet hiver et par la floraison hâlive de quelques plantes, telles que le Polygala à feuilles de Buis (Polygala Chamæbuxus L.), la Gentiane printanière (Gentiana verna L). et autres trouvées en fleurs au mois de janvier sur le Salève, lu me demandes si l'on ne pourrait pas partir, dès à présent, en quête des espèces précoces spéciales au Bas-Valais. Tu sais en effet que nous avions formé un petit groupe de botanistes émérites cherchant à conserver le feu sacré et faisant chaque année, dans ce but, quelques excursions en Valais. Réduits à un bien petit nombre par les infirmités el la mort, nous aurions grand besoin de nous recruter de nous vivifier par la transfusion d'un sang jeune el généreux: aussi j'aime les nouveaux débutants impaiients et fougueux, comme tu l'es, et nous serons heureux de 
l'avoir comme compagnon dans notre prochaine excursion; mais puisque lu fais appel à ma vieille expérience, je dois t'engager à patienter encore quelque temps. En attendant, je vais te raconter une de nos courses dont le récit pourra te donner quelques utiles directions el le faciliter dans la recherche des plantes que tu aspires à récolter

C'était avant l'arrivée officielle du printemps, au commencement du mois de mars $186 \ldots$, que nous entreprìmes celle excursion pour nous procurer quelques espèces que l'on ne trouve qu'à celte époque de l'année.

Nous partimes à 2 heures par le bateau le Simplon, dont les salons ont de bonnes cheminées dans lesquelles on n'épargne pas le charbon, notre projet était de gagner le même soir Martigny oủ nous avions annoncé notre arrivée, sûrs de trouver bon feu, bon souper et bon lit.

M. Clerc, notre hôle habituel, a un faible pour les botanistes; il les choie d'une façon toute particulière, aussi aucun de nous n'aurait l'idée de chercher l'hospitalité ailleurs que chez lui. Grâce à sa persévérance, à son esprit d'ordre et à son intelligence pratique, il a conquis honorablement une belle position. Il faut lui entendre raconter la lutte de ses premiers débuts, et ses essais de colmatage. Il avait loué, pour un bon nombre d'années, une grande étendue de terrains marécageux à une commune qui n'en tirail aucun profit ; les gens de l'endroit n'ayant nulle idée de ce qu'il avait l'intention de faire, ne lui épargnèrent pas les plaisanteries. Les malins lui demandaient s'il voulait établir un vivier? D'autres lui conseillaient de ménager un vieux sanle, qui pourrait lui servir de refuge en cas d'inondation, etc. Quant à lui, laissant dire, il faisait exécuter des fossés larges et profonds en rejetant la 
terre au contre, puis il uilisait l'eau de la Dranse, dont le limon fertilisant avait bientôt rempli les fossés qu'on ridait de nouveau lorsqu'ils b́taicnt pleins. De cette manière, il forma en peu de temps des ilôts carrés, suffisaminent élevés an-dessus du marais, et qui lui donnèrent de magnifiques récoltes en té el en maïs. Ce fut son tour de rire de la stupéfaction de ses voi-sins. En altendant, le branle élait donné, et maintenant les environs de Martigny sont en grande partie rendus à la culture, grâce au colmatage qui utilise les détritus du Rhòne el de la Dranse obligés de réparer, de cette façon, les dégals qu'ils commellent trop souvent. Notre brave ani nous fit, un soir goûtcr de ce fameux vin des Marques, qui se réculte sur les coteanx brûlés dominant au midi la vallée de Martignny, el où l'on trouve un cerlain nombre de plantes rares cl méridionales. Sealement, c'est un vin dont doivent se défier les botanistes qui tiennent à se lever matin.

Le lendemain, de honne heure (nous n'avions pas bu du vin des Marques la veille), nous partons pour rraverser le Rhône sur un mauvais pont de bois plus solide cependant que lant d'antres que le fleuve se plait à emporter dans ses débordenients; puis nous gravissons le chemin qui conduil aux mallieureux villages de Branson et de Fouly dont les habitants ont élé ruinés par les deriières inondations.

Laissant à notre droite Branson, nous nous dirigeons vers les rocher's des Folateires qui forment l'angle saillant de la montagne et qui déterminent le brusque contour que fait le lihòne presque à angle droit, en face de Marligny. A quelques pas du village, nous trouvons sur do petits lalus herbeux, en plein soleil, une des plus rares plantes de la Suisse cl des plus printaniètes en même temps. C'est la Gagée des ro- 
chers (Gagea saxatilis Koch, ou Ornithogalum Bohemicum. Gaud.), mignonne petite plante qui se cache dans le gazon et qu'on a de la peine à disling̣uer quand ses fleurs éloilées, d'un janne doré, ne sont pas encore ouvertes. Celle cspèce ne se trouve pas ailleurs en Suisse, sauf à Sion, sur les rochers de Valère où je l'ai découverte, il y a quelques années. En continuant du côté des Folateires, on arrive à des pelouses où foisonne le Bulbocode du printemps (Bulbocodium vernum L.). Cette plante méridionale ne se trouve en Suisse que dans celle localité et dans un verger du village de Mivellaz au bord du Rhòne ". Elle r'essemble au Colchique d'automne dont elle diffère par sa floraison printanière, par ses feuilles paraissant avec la fleur et par sa corolle divisée jusqu'à la base. Sur les rochers on trouve de beaux échantillons d'une fougère, le Cétérach officinal (Ceterach officinarun C. B.) en bel état de fructification.

Après avoir fait une belle provision du Bulbocode, dont il est difficile d'arracher les bulbes profondément enfouis, il fallut songer au relour; mais nous prîmes l'engagement de revenir plus lard pour trouver des espèces moins précoces, et qui pour la plupart ne so trouvent pas aux environs de Genève.

A 11 h. $1 / 1$, nous prenons le train qui vient de Sion, et qui doit nous conduire à Evionnaz où nous voutions nous arrêter. La saison trop peu avancée ne nous permit pas de faire une halıe à Vernayaz où nous reviendrons plus lard, pour y récolter quelques plantes qui croissent dans cette localité. Noublie pas de vi-

${ }^{1}$ Dès lors on l'a découverte en quantité près des Mayens de Sion, sur la montagne en face de cette ville de l'autre côté du Rhône et sur la colline de Saint-Léonard. 
siter les gorges du Trient qui débouchent dans la vallée par une déchirure étroile et sombre le long des parois de laquelle on a zonstruit un sentier suspendu qui permet d'en visiter les lénébreuses horreurs. La vue du noir torrent, encaissé entre ses roches perpendiculaires, rappelle l'histoire mélancolique des cerises de Gneuroz, si bien racontée par Eug. Rambert, dans les Alpes suisses et qui fait connaitre l'existence rude et périlleuse de ces hommes intrépides qui travailtent au flottage des bois. En traversant le torrent, on trouve sur la rive droite un sentier escarpé, minialure de la Gemmi, conduisant au hameau de Gueuroz et le long duquel se troureni quelques bonnes espèces d'unc floraison plus tardive.

Depuis le sommet de l'éperon qui forme le contrefort de la montag̣re, on domine la vallée du Rhône et l'on comprend tout l'avantage des hommes de Salvan et de Fin-Hauts qui depuis celte forteresse naturelle purent facilement, à l'aide de leurs carabines, décimer les Bas-Valaisans à l'époque de la guerre civile qui ensanglanta ce malheureux cauton. Les nouvelles montagnardes de Ch. Du Bois contiennent, sous le titre de Trient, un récil dramatique et émouvant de ce triste épisode de l'histoire contemporaine de notre pays. Si l'on rencontre encore passablement de ces pauvies créalures hétéroclytes et à moitié sanvagres qu'on appelle crélins, on n'a pas, heureusement. la chance de se Irouver nezà-nez avec le féroce Rôdi, l'un des acteurs le cette narration. Dans ce même volumse brille une charmante nouvelle intitulée: les cloches de Salvan, qui inspire le désir de visiter ce beau village anquel on arrive par un sentier bien élabli el bien entretenu qui serpente sur le flanc escarpé de la monlagne, au moyen de nombreux lacets. Celle nouvelle route aussi facile que pit- 
toresque, construite sur la rive gauche du Trient, traverse les villages de Salvan el de Fin-Hauls et conduit à Valorsine et à Chamounix en rejoignant le chemin de la Têle Noire; les rochers en place, par leur surface striée et polie, témoignent de l'action de l'ancien glacier de Valorsine qui occupait toute cette vallée.

A Evionnaz, nous quillons le train, pour prendre la grande roule, obligée de conlourner le vaste delta formé par les éboulements do la Dent du midi qui nnt refoulé, contre les bases de la Dent de Morcle, le Rhône obligé de lutter péniblement dans son lit rétréci el ohstrué par les blocs descendus avec les torrents de boue vomis par la gorge de St-Barthelemy.

Il est probable que l'ancien éboulement de Tauretunum fut produit par la cliute d'une partie de la Dent du midi qui barra le fleuve et détermina la formation dun lac dans la vallée qui remonte du còté de Nartigny. Ce lac ayant rompu les digues qui le retenaient se précipita avec une violence inouie dans la parlie inférieure du Valais et se déversant soudain dans le lac Léman, occasionna les désastres dont parlent nos annales.

Une faible partie de l'immense éventail produit par les éboulements successifs qui descendent de la gorge de St-Barthélemy, a été rendue à la culture; mais la presque tolalité est encore converte de bois de pins qui portent le nom du Bois-noir et qui sont traversés par les bras nombreux d'un lorrent sauvage el capricieux. Sous ces pins sont entassés confusément des rochers charriés par les avalanches de boue comme les blocs erratiques le sont par les glaciers. Nous récoltons dans ce bois le Cornouillier commun (Cornus mas. L.) dont les fleurs printanières paraissent avant les fenilles et qui se couvre en été de baies rouges et acidules qui sont si chères aux enfauts et aux oiseaux. 
La plante vraiment intéressante de celte localité est la Bruycre incarnale (Erica carnea, L.) seule véritable Bruyère que nous possédions en Suisse. Elle abonde dans les localités qu'elle affectionne tout en étant peu commune dans notre pays, tandis que la Bruyère vulgaire (Calluna vulgaris Sal.) se trouve un peu partout. Eug. Rambert a fait un charmante description aussi vraie que poélique de la Bruyère incarnate dans son récil d'une course manquée à la montagne; il donne également dans son second rolume des Alpes suisses, une monographie complètc et des plus intéressantes de la dent du midi qui fait connaître à fond ce massif imposant.

On trouve encore dans le hois noir la Saxifrage à feuilles opposées (Saxifraga opposilifolia L.) descendue de la dent du midi, le Polygala Chamebuxus, $L$. mêlé à la Bruyère rose, l'Ilepatica triloha, D C. à fleurs blanches et roses et la Primula variabilis Goupil, hybride dont les parents (P. acaulis et P. officinalis) se montrent en grand nombre dans celle localité.

Nous laissons à notre droite, de l'autre côté du Rhône, l'établissement des bains de Lavey, triste séjour' qui doil sa réputation, en grande partie, à l'usage des eaux-mères des salines de Bex et surtout au talent des médecins qui ont successivement dirigé le traitement des malades. Puis, nous nous hàtons de gagner St-Maurice pour prendre le train qui nous conduisit à Genève oủ nous arrivâmes engourdis et à moilié gelés grâce aux wagons peu confurlables de l'ouest.

En somme, celte excursion ne nous a pris que deux jours, et bien que nous n'ayons rapporté que cinq ou six espèces, elles méritent par leur rareté une course faite à leur intention. Plus tard, je pourrai te tracer un itinéraire avec une énumération des autres espèces 
qu'on trouve dans le Bas-Valais, an commencement de mai; seulement, il faud'a consacrer à cetle excursion trois ou quatre jours en raison des localités intéressantes à visiler el du grand nombre de plantes à récolter. Je serai heureux, si les détails que je te donne aujourd'hui peuvent l'intéresser et t'inspirer le désir de te livrer à des excursions saines pour la santé, le cœur et l'esprit, sans compter les doux souvenirs quelles te prépareront pour l'époque où l'on aime à se replonger dans le passé.

Mars 1869.

\section{Cher ami,}

En me remerciant des indicalions contenues dans ma lettre, el gràces auxquelles lu as tronvé les plantes signalées, tII m'annonces que tu veux entreprendre une seconde excursion dans le Bas-Valais, et tu me demandes de nouvelles directions. Donc je m'exécute en te donnant sous forme de voyage le r'ésumé d'herborisations faites précédemment.

C'était par une belle malinée de la fin d'arril qre et bateau à vapeur nous conduisit au Bouveret en coloyant la rive de Savoie. La végétation était peu avancée, les noyers el les chalaigniers avaient encore leur livrée d'hiver; seuls, les saules, les peupliers et quelques autres arbres se couvraient de petites fevilles d'un vert tendre et rougeâtre; les prairies et les champs de blé fornaient des tapis d'une verdure velontée; les arbres fruitiers étaient en pleine floraison el les vieux murs des jardins riverains de Thonon el d'Evian étaient couverts de touffes splendides de la Giroflée jaune (Cheiranthus cheiri L.) dont le parfum arrivait jusqu'à nous Le

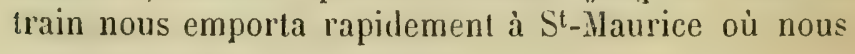


voulions nous arr'̀̀ter, non pour visiter les vieilles églises ct les richesses qu'elles contienrent, bien qu'elles en vaillent certainement la peine, mas simplement pour herboriser. Au pied de la montagne à pic qui domine la gare, le long du sentier qui s'éè̀e d'assise en assise jusqu'à l'ermitage, nous trouvons l'Arabelte des murs (Arabis muralis Bert.), la Tourette (A Turrita L.) la Roquette, (Eruca sativa L.) la Lunetière (Biscutella saxatilis Schl.), le Cranson des rochers (Cochlearia saxatilis Lam), te Baguenaudier (Colutea arborescens L.), le Nerprun des Alpes (Rhamnus alpinus L.), la Laitue vivace (Lacluca perennis $L$ ), la Scorzonère d'Autriche (Scorzonera Austriaca L), el une jolic Fougère (Aspidium Halleri D C.), qui tapisse le rocher près de l'ermilage habité jadis par un malheureux avengle d'un aspect repoussant et d'une dégoutante saleté.

L'année précédente n'ayant pas pu parlir par le bateau, en même temps que mes compagnons, j'avais pris le train dans l'espérance de les rejoindre à St-Mlaurice. Il y avait alor's à cette gare un hargneux personnage toujours en délicatesse avec son collègue de Bex, et les voyageurs avaient souvent à pâtir de l'humeur chagrine et malveillanle de cet individu. Ce jour là, il fit arrêter notre train pendant plus d'un quarl d'heure à l'entrée du tunnel de St Maurice, el quand nous arrivâmes enfin, ce fut pour voir s'èloigner les wagons qui emmenaient à Sion les amis que j'étais venu rejoindre. Nous n'obtìmmes d'autre réponse à nos réclamations, bien inutiles du reste, sinon que c'étail une leçon donnée au chef de gare de Bex qui n'avail pas signalé en temps voulu le départ du convoi. Parmi les voyageurs désapointés se trouvaient deux étrangers, lous deux botanistes et liévérends, l'un Américain, l'autre Irlandais; je m’empressai de les enrôler, en sorte que notre bande se 
trouva composée d'un curé balancé de deux ministres, et d'un pharmacien flanqué de deux médecins et (of course) nous fìnes Irès-bon ménage, comme tonjours.

Mais revenons à mon récit. Voulant gagner du temps, nous louons une voilure qui nous conduit à la Barme et à Mivellaz, oủ une halte nous permet de récoller dans un verger au bord dı Rhône, et dans un autre, à droite de la route, de beaux échantillons du Bulbocode printanier (Bulbocodium vernum L.), en feuilles et en fruits; de là, nous poussons jusqu'à Pissevache, oủ nous renvoyons la voiture. Dans les graviers de la cascade nous trouvons quilques espèces alpines descendues avec la chute d'eau, entr'autres le Passerage des alpes (Lepidium alpinum L.), l'Erine des Alpes (Erinus alpinus L.) et contre les rochers á gauche de la cascade, nous pouvors nous emparer d'une superbe louffe de la Vésicaire (Vesicaria utriculata L.)

En continuant du côté du Trient, nous récoltons dans les prairies marécageuses la Primevire farineuse (Primula farinosa L.), puis nous gravissons le sentier rapide qui s'éléve en zigzag sur l'épaulement de la montagne au dessus de la rive droite du Trient. Nous prenons tout en montant l'Arabette des alpes (Arabis alpina L.), le Passerage des rocailles (Lepidium petræum L.), la Drave aizoide (Draba aizoides L.), l'Arabette des murs, le Cranson des rochers el la Lunetière déji signalés, une Saxifrage assez rare (Saxifraga exarala Vill. var. leucantha (Gaud.). La Silène des rochers (Sileno rupestris L.), l'Herniaire glabre (Herniaria glabra L.), la Primevère visqueuse (Primula viscosa Vill.), la Saxifrage cuneiforme (Saxifraga cuneifolia L.), la Véronique fruticuleuse (Veronica fruticulosa L.), et le Lycopode suisse (Lycopodium IIelvetium L.), ces dernières plantes sur les rochers près de Gueuroz; puis dans les 
prairies avant le village, nous cueillons la Lychnide visqueuse (Lychnis viscaria L.), la Corydale bulbeuse (Corydalis solida Raustralis), la Saxifrage bulbifere (Saxifraga bulbifera L.), une des plantes rares de la Suisse et les deux variéles rouge et jaune de l'Orchis sureau, (Orchis sambucina L.)

Dans une de nos excursiuns précédentes, nous étions arrivés par un bean jour de bise au sommel du sentier qui conduit au hameau de Gueuroz, quand j'aperçois contre le rocher une superbe touffe de la Saxifraga leucantha de Gaudin. Un de nos compagnons, qui l'avait aussi flairée, s'apprête á contourner le mamelon pour la saisir depuis le haut; mais grâce à un effort désespéré pour me dépétrer des ronces qui barraient le chemin, et qui prélevèrent un tribut sur ma blouse et sur mon épiderme, je réussis à détacher la louffe au moment où s'allongeait la main de mon infortuné compétitcur. Furienx, il se redresse, dominant le rocher et commence une harangue ex abruplo, un véritable quos ego!.... Iorsqu'une traitresse raffale de bise lui enlève son chapeau de paille - un courre-chef tout neuf - le fail tourbillonner dans le précipice el le jette sur des sapins suspendus au dessus de l'abìme. Gràce à un magnifique foulard rouge on improvisa un turban qui donna à notre ami une figure tellemenl turque, qu'unc bande d'enfants qui venaient sur la route de Martigny, s'enfuil en poussant des cris de terreur, et que deux mulets attelés a un des chars primitifs du pays firent, à sa vue, un écart qui envoya le véhicule el les natuels qu'il portail, dans $u n$ des fossés du bord de la route. Peul-être, un jour, quelque ornithologue téméraire dénichera ce fameux chapeau transformé en nid par les oiseaux, et en fera le sujet d'un mémoire sur une industrie nouvelle due à l'instinct per- 
feclionné de la gent emplumée du Valais; mais revenons à notre excursion. Le soir, en arrivant a llarligny, nous commençons par soigner nos richesses, c'est-à-dire, que nous empilons nos plantes entre des feuilles de papier gris serré par des cartons au moyen de courroies, puis nous faisons honneur au souper préparé par notre ami Clers.

Seconde journée. Le lendemain, nous gravissons la colline de la Bâtia et nous récolıons la Pulsatille penchée, variélé rema:quable de la Coquelourde ou Pulsatille commune (Anemone Pulsatilla L. var. nutans Gaud.) L'auteur de la Fiore helvélique était un excellent observateur et ses descriptions, remarquablement faites, sont pour le latin, ce que sont pour le français, les descriptions de la Flore de De Candol! Gaudin admet deux formes, la Pulsalille commune et la Pulsalille penchée; celle-ci se reconnait à ses fleurs plus petiles, penchées; ses pétales sont d'un violet foncé et noirâtre, plus cour's et plus obtus; elle fleurit plus tard que l'autre.

La Pulsalille commune croît dans les cantons de Zurich, dArgovie el de Vaud, près de la Sarraz. Elle éait, jadis, abondante à Promenthoux, le long du lac: mais l'ancien propriétaire, qui n'aimait pas les botanistes, fit tous ses efforts pour détruire la plante en houleversant la localité, el les travaux exéculés récemment ont fait disparaitre le peu d'échantillons qui avaient échappé à la proscription. Le jardinier acluel a pu en sauver deux pieds, qu'il conserve précieusement et avec lesquels il espère pouvoir repeupler les berges du lac, dont elle faisait autrefois l'ornement.

Près des ruines du chàleau de la Bàtia végètent quelques touffes de l'Oxytrope champêtre et de l'OxyIrope poilue (Oxytropis campestris D C. et 0 . pilosa D G.); 
sur les rochers sétalent les ligns frèles d'une Aspérule, voisine de la commune appelée vulgairement Herbe à esquinancie, et dont elle diffère par ses corolles plus allongées; elle a été llécrite par Ténore sous le norn d'Asperula flaccida; ça et là, la Véronique couchée (Veronica prostrata L.), épanouit ses grappes de fleurs d'un bleu azuré, et la Laiche lustrée (Carex nilida Ilosi), végète dans les interstices des rochers avec les touffes de la Stipe chevelue (Stipa capillata L.), et de la Slipe plumeuse (S. pennata L.), qiii sont encore peu avancées.

En suivant les sentiers suspendus au flanc de la monlagne, on traverse le vignoble des Marques où crồt un arbrisseau rare pour la Suisse, et qui habite plulôt les contrées méridionales; c’est le Sumac Fustel, vulgairement Arbre à perruque (Rhus Cotinus L.). Là, nous voyons les pousses de l'Orobanche des sables (Phelipaea arenaria Walp ), parasitu sur les racines de l'Armoise champètre, des roseltes radicales d'une Oreanelte (Onosma Stellulatum W. et K.), dont M. Boissier a fait une espèce sous le nom de Onosma helvelica, et d'autres plantes plus tardives. Colte colline donnait asile a un grand nombre d'especes qui aiment ies expositions chandes et sèches, et que l'envahissement de la vigne Iend à faire disparaitre peu à peu. Après-midi, nous nous meltons en route pour Brinson, et nous cueillons en passant une formt pariculière du T:issilage Pétasile, dont les fleurs sont presque toutes femelles (Tussilago Petasites hybrida L.). Gelte plante croìt en abondance le long des canaux d'irrigation de la Dranse avec le Ceraiste des champs (Cerastium arvense L.) ; au bord de la route qui suit le Rhòne, nots prenons des échantillons du Saule fragile (Salix fragilis L.), dont les fleurs sont déjà passées, mais dont les feuilles sont en bon élat. 
Après avoir traversé le pont de bois, nous montons à Branson pour prendre dans le village lo Cerfeuil sauvage (Anthriscus sylvestris L.) et dans les vignes la Calepine de Corvin (Calepina Corvini L.), plante méridionale erratique, paraissant et disparaissint suivant les cultures. Les rignes de Branson ont envahi les localilés aimées des anciens botanistes, et pourchassé les nombreuses espèces rares el intéressantes qui jadis régnaient en souvcraines, paurres chères flıurs détruiles par la cullure des espèces domestiques, comme les Indiens Peaux-Rouges par les progrès de la race blanche. Le vin de ces collines, lorsqu'il est bien soigné, ne le cède en rien aux bons crus étrangers; les plans, originaires pour la plupart du Cortaillod ou de la Bourgogne, ainsi que quelques-uns du pays, tel que le Baillot, donnent un vin rouge et génércux, lor'squ'ils appartiennent à des propriétaires intelligents qui ont remplacé les anciennes: oulines par les méthodes consacrées par l'expérience dai s le canton de Vand. Malheureusement, il n'en n'est point ainsi à Branson, et je n'ai jamais bu de drogue plus infernale que du soidisant vin qui nous fut vendu très-cher par un des habitanls d: l'endroit; c'était un liquide troublr, d'une couleur fausse, d'un goût de moisissure combiné avec une saveur acre et acide, amenant une constriction de la gorge, telle que la déglutilion en était impossible. Ileureusement le village possède unc fontaine abondante, dont l'eau claire et limpide rafraichit agréablement le hotanisle altéré.

Les gens de Branson el de Fouly, énervés depuis longtemps par les funestes émanations des marais du Rhône, perdent avec leurs forces physiques le peu d'énergie morale et d'intelligence dont ils sont doués. Devenus paresseux e: misérables, ils ne travaillent plus 
leurs vignes, et ils finissent par les vendre aux habitants de la vallée de Bagnes, race montagnarde devenue énergique et forle par la lutte incessante qu'elle est appelée à soutenir contre les éliments. Il arrive pour cet endroit ce qui s'est passé dans le vignoble de Sierre. Le plan nommé Arèse et dont l'origine est inconnue, donne un vin dur et désagréable quand on le buit dans la plaine; mais les montagnards d'Aniviers, qui sont devenus possesseurs de presque toules les vignes, transportent les récoltes dans leurs hautes vallées, les soignent convenablement; alors ce vin médiocre se transforme el donne ce fameux cru du glacier, dont la réputalion n'est point usurpée, surtout quand on peut le déguster dans le haut de la vallée et chez les notabilités.

En quittant Branson pour se diriger du côté des Folateires, on trouve une plante qui ne se rencontre pas ailleurs en Suisse: c'est l'Hélianthème à feuilles de saule (Hélianthemum salicifolium L.), croissant sur les pelites pelouses qui bordent le sentier. Le long des chemins, dans les vignes et dans les cullures, nous faisons une ample moisson des espèces suivantes: deux Adonis (A. aeslivalis L. et A. flammaea Jacq.), la Tourelte glabre (Turritus glabra L.), l'Arabette auriculée (Arabis auriculata Larn.), le Vélar de Suisse (Erysimum Helvelicum D C.), la Roquette (Eruca sativa L.), le Pastel (Isatis tinctoria L.), la Cameline sauvage (Camelina microcarpa Andrz.), une petile Pensée naine (Viola tricolor L. var. minima, Gaud), une Potentille (Jotentilla verna var. cineraea Gaud), la Trigonelle de Monlpellier (Trigonella Honspeliaca L.) rare dans cette localité, la Gnavelle verticillée (Ścleranthus verlicillatus Reich.), la G. vivace (S. perennis L.), le Salsifis majeur (Tragopogon major Jacq.), le Podosperme dé- 
coupé (Porlospermum laciniatum D. C.), le Myosotis roide (Myosolis stricta Linck) qui se distingue du M. des collines par la brièveté de ses pédoncules, ses calices fermés et la petitesse de sa corolle, la Véronique du printemps (Veronica verna L.), la V. précoce (V. praccox L.), la V. a trois feuilles (V. triphyllos L.), et l'Asperge officinale sauvage (Asparagus oflicinalis L.). Dans les endioits herbeux, nous retronvons la Pulsatille penchée, la Corydale bulbeuse, le Ciéraiste des champs, la Saxifrage bulbifère, IOrchis sureau, le Bulbocode du printemps et la Laicho lustrée. En s'élevant au-dessus du chemin, sur les pentes de Joux-Brulée, on trouve l'Armeria (Silene Armeria L.), la Lychnide des jardins (Lychnis Coronaria Desr.), el une Irèsrare petite plante qui se cache dans la verdure, le Gaillet du Piémont (Galinm Pedemontanum All.). Sur les rochers et les pelouses sèches végètent le Paturin dur (Selerochloa dura, P. B.), le P. mignon (Poa concinna Gaud.), le Brome squarreux (Bromus squarrosus L.), et le Cétérach (C. officinarum G. B.). Dins les bois, avant d'arriver aux Folateires, commence à fleu. rir le Pigamon puhescent (Thalictrum pubescens Sch.). Enfin sur les pentes qui forment l'angle saillant de la montagne se trouvent en abondance l'Adonis du printemps (Adonis vernalis L.) qui épanouit ses larges fleurs d'un jaune doré, l'Arabelle des murs (Arabis muralis L.), la Lunetière des roches (Biscutella saxatilis Schl.), la Violette des sables (Viola arenaria D C.), l'Oxytrope de IIaller (Oxytropis Halleri Bung.), la Scorzonère d'Antriche (Scorzonera Austriaca L.), et si l'on descend vers le Rhône on verra la Vésicaire (Vesicaria utriculata L.) el l'Ephedra Uvette (E. distachya L.) rare et curieuse conifère, qui ne se rencontro guère que sur les côtes de l'Océan et de la Néditerra- 
née. Quelques auteurs font de notre plante une espèce particulière sous le nom de Ephedra Ilelvelica C. A. Meyer, elle existe à Saillon et en abondance sur les rochers de Valère et de Tourbillon.

Faligués de notre course, pliant sous le faix de nos richesses accumulées, nous regagnons Martigny où nous arrivons le soir altérés et affimés; avant tout, il faut soigner la récolte, et e'est dans celte opération que se traduisent les aptitudes diverses des botanistes dans l'art de mettre en presse le produit de leur chasse. Les uns s'y prennent tout à la bonne, les autres fignolent, arrangent avee un soin minutieux chaque échantillon, les imprévoyants empruntent du papier, etc., puis lis cartons sont duement serrés et ficelés. Enfin le souper réunit la bande qui retrouve son entrain et sa gaielé en résumant les incidents de la journée et en faisant le plan de carnpagne pour le lendemain.

Troisième jour. - Le temps est magnifique; aussi après un déjeuner pris à la hâte, nous partons avant six heures, en prenant l'ancienne route qui cotoie le pied de la montagne, sur la rive gauche du Rhòne. Les arbres en fleurs sèment au vent leur neige odorante dont le frais et doux parfum est dominé par la senteur amère et pénétrante du Cerisier Mahaleb. Le long des haies abonde un Sisymbre nommé vulgairement la Sageșse du Chirurgien (Sisymbrium Sophia L.), aussi commun dans le Bas-Valais qu'il est rare aux environs de Genève où il n'a été signalé que sous les voûles du Salève.

La chaussée moderne qui suit le centre de la vallée, parallèlement a la voie ferrée, n’offre qu'un long ruban monotone, bordé de fossés remplis d'une eau croupissante, el entouré de plaines marécageuses dans lesquelles patdugent de rares troupeaux de vaches mai- 
gres et petites avec des mères juments et leurs folatres poulains. Les peupliers, les saules et les aulnes qui courrent ces tristes solitudes ont tous un air désespéré avec leurs troncs pencliés et leurs branches inclinées dans la même direction, par le fait du violent courant d'air qui souffle presque constamment en remontant la vallée jusqu'à Sion.

En revanche l'ancien chemin, qui suit toutes les sinuosités du pied de la montagne, offre bien plus d'attrait et de variété tout en permeltant de faire une riche moisson de plantes intéressantes. Un jour, je suivais cette roule avec le docteur D., quand je le vois s'arrêter el examiner de petites plantes qu'il venait de prendre le long d'une haie avant d'arriver au village de Charrat; deux d'entre elles nous étaient bien connues; c'était le Tabouret perfolié (Thlaspi perfoliatum $L$.) et l'Holostée en ombelle (Holosteum umbellatum $L$.); quant à la troisième, nous reconnûmes après examen que c'était la Drave des murailles (Draba muralis $\boldsymbol{L}$.) fort rare pour la Suisse, et indiquée par Gaudin comme croissant au pied du Mont-0ttan, avant d'entrer à Maltigny ; cherchée inulilement dans cet endroit par tous les botanistes, elle avait été, en désespoir de cause, reléguéc au nombre des plantes fabuleuses du Valais. C'élait donc une loralité nouvelle que nous venions de découvrir, el comme la plante s'y trouvait en abondance, nous en fìmes une bonne provision. Arrivés le soir à Sion, nous attendions nos paquels de papier que la diligence nous apporta en même lemps qu'clto déposait tout un attirail annonçant la venue d'un confrère en botanique. En effet, nous royons paraître, un peu plus tard, notre digne ami, M. J.-M., qui nous dit d'un air narquois : "Ce n'est pas tout de se lever de bonne heure, il faut encore arriver à temps. „Ce disant, il 


\section{5}

exhibe sa boîte pleine d'une Tulipe que nous comptions récolter le lendemain matin. Cetle belle espèce méridionale, qui n'exisle en Suisse nulle part ailleurs, est connue sous le nom d'Eil du Soleil (Tulipa OculisSolis, St-Am.); néanmoins, on a prétendu que notre plante de Sion et de la Savoie, diffère de celle du midi de la France et on lui a donné le nom de Tulipa Didieri, Jord. Notre malin compétileur, ayant vu nos cartables sur la diligence, s'était fait descendre à l'endroit de la Tulipe el s'était emparé de tout ce qu'il avait pu trouver. En bon prince, il nous en offrit quelques échantillons que nous n'cûmes garde de refuser, nous promettant de faire le lendemain de nouvelles recherches, ce qui fut effectué avec succès; nous pûmes en faire une honne provision el lui rendre ce qu'il nous avait donné. Mais tandis qu'il nous plaisantait, nous ouvrons nos hoîtes el lui montrons la plante que nous avions déconverte à Charral, celle-là même qu'il avait taxée de fabuleuse; ce fut notre tour de jouir de sa stupéfaction el de nous montrer grands et généreux; il reçut sa part arec des indications précises sur la localité; notre ami n'en dormil pas el à l'aube du jour il était en roule pour récolter lui-même cette affreuse pelite drôlcrie (c'est ainsi qu'il désigne les plantes qu'il affeclionne), et il put s'assurer que nous n'avions pas tout dévasté. Du reste, c'est une plante erratique el capricieuse dont nous avons eu peine à retrouver quelques maigres échantillons depuis cette époque. Ce nom de Charrat remémorait au docteur D. un souvenir plein d'émotion; aussi ne passe-t-il jamais pris de ce village sans faire une visile au syndic de l'endroil, auquel il dût, après Dieu, la vie de sa famille, dans une de ces inondations si fréquentes en Valais. Mais revenons à notre voyage, car je m'apercois que je me laisse entrainer par le charme des souvenirs. 
Après avoir passé les deux villages de Charrat, on cotoie une suite de collines exposées en plein soleil, et sur lesquelles nous trouvons bon nombre de plantes dont voici l'énumération. Sur les rochers, les pelouses et les maigres cultures se plaisent l'Adonis du printemps, la Cameline sauvage, la Sabline fasciculée, (Alsine fasciculata, M. et K.) l'Oxytrope de IIaller et l'O. poilue, l'Astragale Esparcette (Astragalus Onobrychis, L.), l'A. de Montpellier (A. Monspessulanus, L.) encore peu avancée, la Peselte de Gérard (Vicia Gerardi, D G.), la P. fausse Esparcelte (V. Onobrychioides, L.) la Joubarbe aranéeuse (Sempervivum arachnoideum, L.), et la J. des toits (S. lectorum, L.), ces deux espèces couvrent tous les coleaux du Bats-Valais, clles ne sont pas encore flcuries et la première paraît être une variété remarquable, sinon une espèce distincte du type qui habile les Alpes élevées. Signalons encore le Gnaphale jaunâtre (Gnaphalium lıteo album, L.), l'Epervière laineuse (Hieracium lanalum, L.), l'E. mouchetée (H. pictum, Schl.), le Salsifis majeur, la Scorzonère d'Autriche, la Véronique couchéc, le Thésion intermédiaire (Thésium intermedium, Schr.), l'Agrostide interrompue (Apera interrupta, P. B.), la Koelérie du Valais (Koeleria Valesiaca, Gaud.), la Fétuque du Valais (Festuca Valesiaca, Gaud.), le Brôme squarreux et l'Agropyre glauque (Agropyrum glaucum, R. et S.) Le long des haies el dans les prairies au bord de la route nous récolıons le Pigamon à feuilles élroites (Thalictrum angustifolium. L), le Velar effilé (Erysimum virgalum, Roth.) el le peuplier blane (Populus alba, L.).

Saxon où nous nous arrêtons pour absorber une cruche de bière mousseuse, en allendant le train qui doit nous conáuire à Sion, est célèbre par ses bains dont l'eau minérale a donné lieu à une polémique qui 
dure encore, sur la nature de sa composition; les uns affirment que celte source contient de l'iode en abondance, d'autres prétendent qu'il n'y en a point, d'autres enfin croient que cette substance minérale ne s'y trouve que d'une manière intermittente. Il y a déjà bien des années, au moment oú l'on commençait à parler de la découverte de l'iode dans ces caux, le professeur de Charpentier me mon!ra chez lui une pierre qui en contenait une assez forti proportion et qu'il avait délachée de la colline au pied de laquelle est construit l'élablissement des bains: le savant géologue affirmait que $\mathrm{co}$ banc de calcaire marin se retrouve dans la vallée de Bagne et à Saillon de l'autre côté du Rhône: il ajoutait qu'il existe à Saillon une autre source contenant de l'iode.

Le calcaire en question est poreux, et les anfractuosités en sont tapissées par une substance pulvérulente d'un jatne orangé el qui exhale une odeur d'iode des plus caractérisée; j’en ai apportí des échantillons cha. que fois que je suis allé a Saxon. Pour ma part, je suis convaincu que l'eau de Saxon contient de l'iode, et que c'est à la présence de celle substance éminemment active que lon doil attribuer les nombreuses guérisons des maladies des os el des articulations, ainsi que des lé:ions variées qui dépendent du vice scrophuleux. Malheurousement le gouvernement du Valais a laissé s'élablir à Saxon l'immorale industrie des jeux de hasard et la déplorable société qui s'y donne rendez-rous, chasse la plupart des personnes qui ne se rendaient là que pour leur santé.

Nous avons quelque velléité de monter au village d'Iserabloz, situé à une assez grande élévation au-dessus de Riddes et sur le flanc de la dent de Nendaz; là, croit une plante exirèmement rare le Sisymbre de Hongris. 
(Siysmbrium pannonicum Jacq.), qui ne se trouve nulle part ailleurs en Suisse. Mais la course est lon ue et fatigante, el nous nons décilons à prendre le train qui nous amène à Sion à 10 heures el demie. - Les i irilles murailles crénelées qui prolégeaient la ville sont aujourd'hui éventrées; les lours qui les flanquaien! sont en partie écroulées, mais les bâtiments massifs de: l'ancienne église de Valère et les ruines du chàtean des Princes-Evêques qui couronnent les mamelons jumeaux, au pied desquels est bàtie l'ancienne cité épiscopale, lui conservent toujours son aspect pilloresque ol belliqueux.

Sur les rochers de Valère du côté du Rhône, s'étalent les raquettes épineuses du Figuier d'Inde (Caclus Opuntia L., Opuntia vulgaris Mill.), originaire de l'Amérique, naturalisé dans l'Europe méridionale d'où il remonte dans le Tessin el jusque dans le Valais, qui est sa slation la plus septentrionale. Malheureusement il n'est pas encore fleuri, et puis, ce n'est pas une plante commode à sicher ; en revariche, les murailles do rochers sont tapissées d'Iris en fleur's (Iris Germanica L.); cà et là nous trouvons quelques beaux échantillons de Clypéole (Clypeola Jonthlaspi L.), rare plante méridionale, dont il restequelques traces au pied des virux murs du chàteau de Tourbillon et qui est plus fréquente près de l'ermilage de Bramois. Le Pastel (Isatis tinctoria L.) est abondant là, comme dans les rnvirons de Sion, et nous prenons une variété de Cerfeuil (Anuhriscus Cerefolium Var. trichosperma Koch.). Ontre les plantes dont elle est ornée, cette localité nouss offre un altrait tont spécial dans la personne d'un de nos compatriotes, possesseur d'une charmante maison de campagne au bord du Rhône, el chrz lequel nous trouvons toujours la plus gracieuse el la plus cordiale hos- 
pitalité. Un jour par un chaud soleil de mai, après avoir parcouru les rochers brûlants de Valère, nous allons, comme d'habitude, frapper à la porle de notre ami qui se trouvait absent. Elendus sous un noyer nous cherchions à nous rafraîchir en discutart le breuvage auquel on accorderait la préférence en arrivant à Sion ; l'un vantait la bière mousseuse, l'autre préférait le muscat de St-Léonard, un autre recommandait l'eàn fraìche. enfin le plus excentrique exaltait les vertus d'un mélange d'eau de seltz, de sirop et d'absinthe; la discussion s'animait, les gosiers se dessèchaient, quand apparaît, comme le Dens ex machina, notre ami C. qui nous met tous d'accord en nous versant du vin d'Yvorne accepté avec le mème entrain qu'il était offert et déclaré à l'unanimité hautement préférable à tout ce qui avait fait la discussion sous le noyer.

Entre la colline de Valère et celle de Tourbillon, nous trouvons, non sans peine, quelques paures échantillons de la fausse Gesse (Vicia lathyroides L.), que nous possédons, quoique tonjours rare, aux environs de Genève; malgré toutes nos recherches, il nous est impossible de tomber sur la Chelidoine cornue (Chelidonium corniculatum L.), plante méridionale qui se trouve par ci par lá dans les moissons; en revanche, nous prenıns l'Ornithogale des rochers (Gagea saxatilis Koch.), en bons fruits; elle habite au pied des rochers sur lesquels sont construits les battiments de Valère et que décorent des touffes de la Giroflée jaune (Cheiranthus Cheiri L.). Sur la colline de Tourbillon nous voyons l'Ephedra (distachya ou Helvetica) et un Iris rare (Iris virescens D. C.) qui ne se trouve que dans une enceinte fermée où il est impossible de pénétrer; près du château, la pelouse esı tapissée de la Trigonelle de Montpellier, du Paturin dur et du Patu- 
rin mignon, dont il a clé, question précédenment. Le soir, en nous promenant aux environs de la ville, nous pûmes récolter de beaux échantillons de l'Adonis d'été de la Tulipe œeil de soleil. Malheureusement la localité dans laquelle celle belle plante sétait établic et qui d́tait autrefois occupée par des champs de céréales, se convertit en prairies artificielles, ef, par suite de ce changement de culture, la plante tend à disparaitre, mais espérons qu'elle ressuscitera lorsque le blé reprendra son tour.

Quatrième journée. - Toujours favorisés par le heau temps, nous partons de grand matin pour faire une rapide excursion sur la colline de Honiorge, qui présente à peu près la même végétation que la colline de St-Léonard, siluée à moilié chemin de Sierre. Your ne pas tomber dans des répétitions, je me bornerai à ilire que notre récolte se composa des plantes que nous avions dejả prises à Branson et ailleurs, auxquelles s'ajoutèrent la Bugrane ả petites feuilles (Ononis Columnæ Ail.), le Buplèrre à feuilles rondes (Buplevrum rolundifolium L.), le Gaillet de Vaillant (Galium Vaillantii D C.), l'Androsace à grandes fleurs (Androsace maxima L.), enfin une des plus rares plantes de la Suisse, l'Avoine de Gaudin (Avena Lœfllingiana Gand; Trisetum Gaudinianum Boiss.). Getle jolie graminée avait ¿té nommée par Linné : Avena Lœlflingiana, et Koch, dans son Synopsis, l'a appelée Aveua Cavanillesii, sát description est conforme aux échantillons de la plante suisse, mais la figure qu'il cile apparlient à une uspèce spéciale à lEspagne à laquelle M. Edm. Boissier conserve le nom de Trisetum Loeflingianum Pol.

A temps pour prendre le train qui part de Sion à 10 h. $1 / 2$, nous descendons à Villencuve pour monter sur le bateau à vapeur, oủ nous savourons toutes les 
jouissances d'une navigation paisible sur notre beau lac et de la vue toujours splendide et merveilleuse des montagnes et des Alpes, donl le panorama se déroule le long de la rive gauche, depuis les pics neigeux de la Dent du Midi aux glaces étincelantes du Mont-Blanc et de ses satellites.

Chr. F., Dr- $\mathrm{N}$.

P. S. - Je reçois la visite de M. J. Muret, de Lausanne, qui m’a donné des échantillons du Bulbocode printanier, trouvés par lui en mars 1869 sur la colline de Saint-Léonard, où il n'avait pas encore été signalé ; il paraît assez abondant, surtout la variété à fleur blanche. Puisque je parle de Saint-Léonard, je dois ajouter que c'est dans cette localité, sur la crète des rochers qui dominent la route de Sierre, que croit la Renoncule graminée (Ranunculus graminaeus, L.), plante rare qui n'existe pas ailleurs en Suisse; elle habile de préférence le midi, mais elle se retrouvo près de Lyon, aux environs de Paris el en Bourgogne. Malheureusement la vigne envahit tout à Saint-Léonard, et comme la terre végétale n'abonde pas sur celle colline, les propriétaires vont râcler le maigre terreau et la couche de mousses qui recouvrent les rochers, et détruisent ainsi la $R$ snoncule, qui, jusqu’à ce jour, avait régné paisisiblement sur ce sol aride et déshérité.

Ch. F. D. M. 
Genève, septembre $187 \ldots$

\section{Cher ami,}

En mellant en ordre des plantes recueillies dans le Haut-Valais, j'ai été pris d'un remords en pensant que jai oublié la promesse qu: je t'avais faite de t'indiquer les espèces les plus intéressantes qui fleurissent, en étć et en automne, dans le Bas-Valais que tu as exploré au printemps. En feuilletant l'herbier il faut un effort d'imagination pour reconstituer le port, la physionomie el le coloris de res pauvres flenrs déformées, applaties et desséchées dans leur linceuil de papier. Néanmoins, leur vue fait instanlanément vibrer les cordes de la mémoire, alors les fleurs du souvenir, ces flcurs toujours jeunes, vivaces et brillantes, s'épanouissent en foule évoquées par les pàles fantômes de leurs sœurs de l'herbier. On revoit les localités oủ elles furent cueillies, les amis en compagnie desquels on se trouvait, on se rappelle les discussions, les causeries à bìtons rompus, les joies, les désappointements, les atternalives de pluie et de soleil, toutes les péripéties qui faisaient le charme de l'excursion.

Si le champ de bataille de l'herbier fait éprouver une sorte de mélancolie à la vue de res rnalheureuses plantes défigurées et presque méconnaissables, au moins il ne soulève pas ce sentiment de répugnance et d'horreur que l'on éprouve à la vie des blessures sanglantes et des plaies hideuses, à l'ouie des cris de douleur 
el des plaintes déchirantes des champs de bataille humains.

Le Grand Architecle de l'Univers se manifeste dans la nalure et dans ses ouvres, comme un Dieu de paix et d'amour; mais les ambitieux et les conquérants ont besoin, pour tromper leur conscience, de se faire un Dieu à leur image, un Dieu sc plaisant aux hécatombes humaines, qu'ils voudraient rendre solidaire des mas. sacres qu'ils commettent en son nom.

Du reste, ce qui me console un peu de ne pas t'avoir écrit plus tôt, c'est que j'ai su que tu as moins d'occasion de te livrer' à ton goût pour la botanique, depuis que lu as dû quitter la campagne pour pratiquer la médecine dans un chef-lieu. Pauvre ami! je te plains d'être au service d'une maìtresse égoïste, exigeante et jalouse qui ne pardonne guère les infidélités que lui font ses adeptes à l'endroit de l'aimable Flore, sa gracieuse rivale qui a l'avantage de rester toujours jeune, aimable et attrayante, landis que la vieille Hygie devient loujours plus renfrognée, plus susceptibl: el plus acariâtre. Cependant, cette respectable matrone devrait savoir qu'ello est forte de ses avantages et des droits que lni confère sa lŕgitime position; l'expérience n'at-elle pas prouvé que le vieux praticien ne peuı guère faire autre chose que de la médecine, et qu'il devient passionné pour sa profession, comme le chasseur ou mieus comme le joueur. En effel, !a parlie qu'il engage chaque juur n'est-elle pas riche en émotions, puisqu'elle a pour enjeu la vie d'un malade et pour adversaire l'implacable mort avec sa faux, son double zéro et ses iefaits?

Mais revenons à nos piantes a aiandonnées trop longtemps; celles que l'été nous réservera dans le Bis Valais seront en nombre plus rostreint qu'au début de 
la belle saison, surtout si l'on compare la végéfation de la plaine à celle des, hautes vallées de Bagnes, de Saas el de Zermatt, ainsi que des cols de la Gemmi et du Simplon. Plus tard, je pourrai peut-être te donner quelqnes déliails sur les espèces les plus rares de ces localités, pour le moment je ne ferai que mentionner la Saxifraga controversa Stern, que j’ai récolice en quantité au Pas du Loup, au-dessus des bains de Loësche, avec l'Aquilegia alpina, etc., le Ranunculus parnassifolius, L. en compagnie dautres bonnes espèces alpines, sur le col de la Gemmi.

Puisque je parle de Loësche, je veux te dire que ces eaux si célèbres pour la guérison de cerlaines formes de maladies de la peau, doivent évidemment leur efficacilé à la présence de l'arsenic qui a été trouvé dans 10 limon des réservoirs, il y a déjà plusieurs années, par le Dr Payen, médecin inspecteur des bains de St-Gervais, el par feu Dublanc, ancien économe de la pharmacie centrale des hôpitaux. Je possède la nolice imprimée dans le temps par ces Messieurs et communiquée à l'Académie de médecine. Néanmoins, les médecins de Loësche n'en parlent pas el quelques-uns rraignent même que le public n'en soil instruit. Je regrette que l'analyse que devait faire Pyrame Morin n'ait pas paru et qu'elle soit restée, depuis sa mort, enterrée dans quelque oubliette de sa pharmacie. Comme il voulait employer l'analyse spectrale, il est plus que probable que non-seulement il aurait trouvé l'arsenic, mais certainement encore d'autres éléments, car on ne peut pas expliquer les effets merveilleux de ces eaux seulement par leur température élevée, leur élecIricité el les substances minérales que détaillent les anciennes analyses.

L'Astragalus exscapus L., la Matthiola varia Gaud., se 
Irouven t en montant au Simplon; l'Hieracium alpicola Schl., espèce bien dislincle, couvre les pelits mamelons des palurages aulour de l'llospice avec une foule de plantes qui son! rarss pour nous; mais $j+$ n'en finirais pas si je me laissais aller à une plus longue énumération. Cependant je dois te recommander une excursion soit au Simplon, soit au St-Bernort, non-settlement pour jouir de la générense hospilalité el de la bienveillance cordiale des Révérends Chanoines, mais encore pour récolıer, grâce à leurs indicalions, les espèces spéciales à ces localités.

Peut-être trouveras-lu bien usurpée la réputation de ces famenx chiens qui firurent dans les albums pitloresques ef les Keepiakes anglais avec un flacon pendu au cou el un enfant, tiré de l'avalanche, it cheval sur leur dos. Tandis qu'en réalité ce sont des espèces de bêtes fíroces aux colliers desquels on est obligí d'atacher une lourde bûche pour les empècher de courir sus aux moulons dont ils apprécient trop bien les còtelettes à la minute et les gigols au naturel. L'un d'eux, achelé à Berisal par un étranger, élait en vente pour la seconde fois, son maitre l'ayant laissé pour compte à la vue de la carte à payer pour moulons étranglés et autres méfails $100 m m i s$ en son absence. Au demeurant, ce sont les plus belles bètes du monde, laisant trèsbien dans le paysage el dans la légende, mais avec lesquels il faut avoir le moins de points de contact que possible. Tu sais si j’aime les bêtes, qui me le rendent bien du reste, néanmoins ma boîle de bolanique porte l'empreinte des dents d'un des chiens du Simplon qui en rouiait à mon bras, et celui de l'abbaye de StMaurice a failli me dévorer. Malgré cela, je me garderai bien de mellre en doule ce que Tschudi et d'antres personnes dignes de foi racontent des services 
que rendent aux voyageurs les chiens de l'Hospice du Grand St-Bernard.

Pendant l'été tu devras visiter au moins deux fois les diverses localités que nous avons déjả parcourues; mais je ı'engagerai à faire une pointe, le printemps prochain, jusqu'au creux de Novelle, au-dessus de StGingolph, pour cueillir aans les vergers, le Crocus printanier (Crocus vernus All.), le Perce-ncige (Leucoium vernum L.), la Gagée jaune (Gagea lutea Sch.) el quelques autres plantes de la première saison. Plus tard tu trouveras, au-dessus des maisons de Novelle, le Rhododendron (Rhodendrum ferrugineum L.) qui descend également assez bas vers le lac de Tannay, près de Vouvry, charmante localité, véritable parterre tout émaillé des plus jolies fleurs des Alpes. Au Bouveret, lu pourras trouver l'Anserine rouge (Chenopodium rubrum L.), avec le Chenopodium ficifolium Sm. (Rapin, Muret) ; à Vouvry, dans les marais près de la gare, le Pigamon jaune (Thalictrum flavun L.), le Seneçon des marais (Senecio paludosus L.), le Géranium des marais (Geranium palustre L.), le Rubanier nain (Sparganium minimum Fries), l'Epipactis des marais (Epipactis palustris Cr. arec l'Herminium monorchis R. Br.), dans les sables au bord du Rhône (Reuter). A la porte du Scex, au pied des rochers, la Violette singulière (Viola mirabilis L.), sur les rochers des Joubarbes de formes variées se rapporlant au Sempervivum tectorum L.); mais surtout lu chercheras, en automne, le Cyclamen à feuilles de lierre qui a été trouvé dans celte localité et qui n'élait connu qu'audessus de Roche, de l'autre côté de la vallée.

A St Maurice, n'oublic pas de visiter le trésor de l'abbaye, riche en antiquilés dont la valeur égale la rareté; Messieurs les Chanoines t'en feront les honneur's 
avec !a politesse la plus courtoise, mais garde-toi du chien, Cave canem!

$\mathrm{Si}$ tu as le temps, va voir la grotte des Fées dont tu liras la charmante légende dans le nouvel ouvrrge d'Eug. Rambert sur Bex et ses environs. Puis tu tâcheras de retrouver le Genêt ovale (Genisıa ovata W. et K ) indiqué à la Crolta, entre St-Maurice et outre-Rhòne, par Wlurith et Gaudin, sinon tu te rabatIras sur la Rue odorante (Rula Graveolens L.), la Potentille caulescente (Potentilla caulescens L,), le Sorbier hybride (Sorbus hybrida L.). I'Epervière glauque (Hieracium glaucum All.), l'E. amplexicaule (H. amplexicanle L.) l’E. de Jacquin (H. Jacquini Vill.), le Sesseli bisannuel (S. bienne Cranız). le Trochiscante nodiflore (Trochiscanthes nodiflorus Koch) sur les rochers audessus de la route entre St-Maurice el Pissevache, près d'Epinassey, la Véronique couchée (Veronica prostrala L.), l'Oseille sculifère (fumex scutatus L.), le Spiranthe d'été (Spiranthes æslivalis Rich.) et la Stipe plumeuse (Stipa pennala L.).

En continuant la route lu pourras chercher dans les vergers de la Barme les bulbes du Bulbocode du printemps et cueillir dans le gravier autour de la cascade de Pisse-Vache quelques espèces descendues avec le torrent telle que l'Arabette naine (Arabis pumila L), l'Alchernille des Alpes (Alchemilla alpina L.), la Saxifrage d'automne (Saxifraga aizoides L.), le Tussilage neigeux (Tussilago nivea Vill.), l'Erine des Alpes (Erinus alpinus L.). Sur les rocher's végètent la Biscutelle des rochers (Biscutella saxalilis Schl.), la Vesicaire utriculée (Vesicaria utriculata L), la Valériane trisèquée (Valeriana tripteris L.) et le Houx-fragon (Ruscus aculealus $L$ ):

Vers le Trienı, tu récolteras la Pédiculaire des marais 
(Pedicularis palustris L.), l'Euphorbe de Gérard (Euphorbia Gerardiana L.), le Saule Daphné (Salix Daphnoides L.) !a Sabine (Juniperus sabina L.) an pied des. rochers et le Céterach officinal (Ceterach officinarum C. B.).

En montant à Gueuroz lu verras l'Arabelle des murs (Arabis muralis L.) en bons fruits, la Vesicaire sur les rochers, la Lychnide visqueuse (Lychnis viscaria L.) dans les prairies, la Sabline trinerviée (Arenaria Irinervia L.) dans les bois, I'Herniaire glabre (Ilerniaria glabra L.) avec la Gnavelle verlicillée (Scleranihus verticillatus Reich) sur les rochers du sommel, la Saxifrage à fleurs blanches (Saxifraga leucantha Gaud.) en montant le long du sentier, la S. étoilée (S. steltaris L.) et la $\mathrm{S}$. cuneiforme (S. cuneifolia $\mathrm{I}_{\text {. }}$ ) sur les rochers humides, la $\mathrm{S}$. bulbifëre (S. bulbifera L.) et la Raiponce à feuilles de Bétoine (Phyleuma betonicafolium Vill.) dans les prairies avec l'Orchis sureau 0 . Sambucina L.) enfin sur les rochers la Primevere visqueuse (Primula viscosa Vill.) et le Lycopode suisse (Lycopodium. Helveticum L.).

Tu feras une halte à Mfartigny dont les environs te fourniront une riche moisson. Avant d'arriver, il faut longer le pied du mont Otlan qui s'étend jusqu'au faubouro de la Bâtia dominé par une colline abrupte sur laq relle s'élève fièrement une vieille tour, débris de l'ancien château-forl des ducs de Savo e. An pied des rochers on trouve l'Arabelte des rochers (Arabis saxatilis L.) toujour's assez rare, le Geranium luisan! (G. lucidum L.) et la Doradille septentrionale (Asplenium septentrionale Sw.) qui ne croit que sur les roches cristallines. Tàche de retrouver la Drave des murs (Draba muralis L) indiquée par Gaudin dans celle lonalité, mais qui n'a jamais été revue. Voici son indicalion: 
«Ad clivum arduum, viæ, qua pagulus seu suburbium la Bâtia intratur impendentem, monti Ottan et Colli les Marques contiguum. „

Sur la colline de la Bâlia croissent la Silène à petites fleurs (Silcne Otites Sm.), l'Oxytropis champêtre $(0$. campestris D C.) et l'O. poilue (0. pilosa D C.), différenles formes de Joubarbes, la Campanule en épi (Campanula spicala L.), l'Euphraise jaune (Euphrasiá Iutea L.), le Brôme squarreux (Bromus squarrosus L.), la Phléole rude (Phleum asperum Vili.), la Slipe chevelue (Stipa capillata L ), etc., etc.

Marligny occupe le point de la vallée où le Rhône fait un coude brusque pour se diriger vers le lac Léman. C'est depuis celte petite ville, où les touristes abondent en élé, que l'on se rend soit à Chamounix par la Forclaz et la Tète-Noire ou le Col de Balme, soit au grand Saint-Bernard ot dans la vallée d'Aoste. Autrefois les sentiers étaient rudes el faligants; maintenant on a élabli des routes à voiture dont l'une, celle du SaintBernard, s'arrète à la cantine de Proz, à 2 heures audessous de l'Hospice el l'autre celle de Chamoniz va jusqu'au grlacier du Trinnt exploilé par une compagnie qui envole velle magnifique glace se consommer it Genève, à Lyon et à Iaris. Avant peu les char's à banc pourront aller directement de Marligny à Chamonix, pour le plus grand agrément des touristes.

Il est de mode chez les clubisles de mangréer contre ces caravanes qui leur gâlent le paysage; je trouve au contraire qu'elles ne font pas mal avec leurs vciles verts et bleus, leur's plaids de loutes couleur's el je ne vois pas pourquoi le touriste n'aurait pas le droit de chevaucher sur son mulet, le long d'une bonne route de monlagne, aussi bicn que le clubisle chevauche sur ses jambes dans des sentiers impossibles. D'ailleurs le piéton saura 
tonjours trouver les vieux sentiers abandonnés, sans compter toutes ces vallées peu connues et ces passages ignorés sur lesquels les nouvelles rartes du club alpin, si belles et si exactes, donnent des indications précienses pour ccux qui veulent fuir les tourisles et qui ne craignent ni le vertige, ni la fatigue.

Autour de Martigny et surtout sur le coteau des Marques se trouvent le Passerage des décombres, (Lepidium ruderale L.), le Sumac Fuslet ou Arbre à perruque (Rhus cotinus L.), la Bugrane à petites fleur's (Ononis columnæ All.) la Potentille dressée (Potentilla recta L.) une Asperule (Asperula longiflora W el K.), et le Peucédane de Venise (Pencedanum Venelum Koch), la Trinie commune (Trinia vulgaris D C.), la Laitue viminale (Lactuca viminea Sch.), la Chrysocome à feuilles de lin (Chrysocoma linosyris L.), l'Immortelle fermće (Xeranthemum inaperlum L.), la Lampourde commune (Xanthium strumarium L.). la Gampanule de Bologne (Campanula Bononiensis L.), l'Orcanelle étoilée (Onosma stellulatum W. et K.), la Ilolène des montagnes (Verbascum montanum Sehr.), la Phelipée des sables (Phelipea arenaria Wal.), l'Eragrostis faux-paturin (Eragrostis poaeoides P. B), I'E. poilue (E. pilosa P. B. ', la Molinie Iardive (Molinia serolina M. el K.), la Koelérie du Valais (Kocleria Valesiaca Gaud.), le Feluque du Valais (Festuca Valesiaca Gaud.), l’0phioglosse commun (Ophioglossum vulgatum $L$.) etc., elc.

L'Ilerborisation de Branson est toujours riche et demande assez de temps; il faut s'armer de patience, d'une grande boite, d'un cartable bien garni el d'une bonne gourde pour apaiser la soif, car il y fait chaud.

Au bas de la colline, contre les rochers, croissent la Joubarbe araneuse (Sempervivum arachnoideum L.), l'Orlaya a grandes fle'ırs (Orlaya grandiflora Hoffm.), 
et le Marrube commun (Narrubium vulgare L.); dans le village, l'Anserine à feuilles d'Obier (Chenopodium opulifolium Schr.), l'A. rouge (C. rubrum L.); le long des senliers, au bord des vignes et des cullures, tu retrouveras les plantes déjà indiquées el d'autres encore telle que l'Astragale esparcette (Astragalus Onobryihis L.), l'A. de Monıpellier (A. Monspessulanus L.), la Vesce fausse Esparcelte (Vicia Onobrychioides), la Gesse spherique (Lathyrus sphœricus Retz.), la Laituevireuse (Lactuca virosa L.), la L Scariole (L. Scariola L.), l'Echinope à tête ronde (Echinops sphorocephalus L.), le Chardon-marie (Silybum Marianum Gaerın.), la Crupine commune (Grupina vulgaris Cass.), la Lampourde (X. Strumosium L.)

Sur les pelouses et les palurages vivent en société l'Achillée lomenteuse (Achillea tomentosa L.), l'A. setacée (A. setacea W. et K.), l'A. noble (A. nobilis L ) et une variétédu Serpolet (Thymus serpyllum var. pannonicus All.). La Grande Absinthe (Artemisia Absinthium L.). et l'Orobanche de l'Armoise (Orobanche Artemisiae Vauch.) préfèrent les cultures et les vignes. En montant vers Joux-Brulée on trouve la Lychnide des jardins (Lychnis Coronaria L.), et de superbes échantillons du Gaillet du Piémont (Galium Pedemontanum L.) (MH. Rapin et Muret). Sur les pelouses rocailleuses vegetent l'Epervière de Le Pelelier (Hieracium Peleterianum Mer.) et l'Hyssope officinale (Hyssopus officinalis L.); dans les bois en arrivant aux Folateires, le Pigamon pubescent (Thalictrum pubescens Schl. et surles rochers au bord du Rhône l'Ephedra en bons fruits, (E. Helvetica Ney.)

Une pointe sur Saillon te fera trouver la Clematite dressée (Clemalis recta L.) rare, l'Amandier (Amygdalus communis L ) en fruits, le Télèphe à feuilles alternes (Teleph um Imperati L.) rare, la Turgenie à larges 
feuilles (Turgenia latifolia Hoffm.) Irouvée une fois par M. Murel, l'Epervière laineuse (Hieracium lanatum Vill.) qui est aussi sur les rochers entre Charrat et Saxon, l'Acore aromatique (Acorus calamus L.) dans les marais, et le Sclerochloa dura P. B.

Je t'engage à suivre l'ancienne route qui suille pied de la montagne entre Martigny et Saxon; tu reverras bon nombre des espèces dejä indiquées el lu récolteras en outre la Bugrane géante (Ononis allissima Lam.), l'Astragale de Montpellier en bon état, l'Esparcelte des sables (Onobrychis arenaria D.C), la Peselte de Gérard en fruils murs, les deux Achillées de Branson, l'Epervière laineuse, l'Epervière mouchetée (Hieracium pictum Sch.) le Gnaphale jaunàre, la Bryone blanche (Bryonia alba L.) fort rare, trouvée près de Saxon, la Véronique couchée (Veronica prostrata L.) el la Prèle rameuse (Equisetum tamosum Schl.)

A Saxon tu monteras en chemin de fer jusqu'à la station d'Ardon où tu verras le Guy croissant sur les Pins, tu chercheras la Buffonic à grosses graines sur les bords de la Morge et tu no craindras pas de gravir la colline de Montorge couronnée par les ruines pittoresques d'un vieux château. Outre les plantes énumérées précédemment, tu verras le Buplèvre à feuilles rondes (Buplevrum rotundifolium L.) la Crupine commune (Crupina vulgaris Cass.), la Centaurée du Valais (Centaurea Valesiaca Jord.), le Micropus erectus L., la Phelipea coerulea Ney., l'Androsace maxima, le Safran cullivé (Crocus Sativus L.), le Froment Faux-Nard (Triticum Nardus D C. Gand.) rare, l'Ivraie raide (Lolium rigidum Gaud ) ete. Honlorges est l'une des localités où croit l'A voine de Gaudin (Trisetum Gaudinianum Boiss.) une des plus rares espèces de la S'risse.

Autour de l'Etang de Montorge croissent la Renonée 
amphibie (Polygonum amphibium L.), l'Orchis laxiflore (0. laxillora Lam.), la Liparis de Loesel (L. Loesclii Rich.), la Massetle à feuilles étroites (Typha angustifolia L.) le Scirpe marilime (Scirpus maritimus L.) la Laiche faux-souchet (Carex Pseudo-Cyperus L.) trouvée par M. Reuter et la Fétuque géante (Festuca gigantea Vill.) etc.

A Sion végètent la plupart des espèces que nous avons déjà désignées, lelles que le Thalictrum fotidum L. qu'on trouve près des Folateires, la Trigonella Monspéliaca L., le Telephium Imperati L., l'Ephedra helvetica Mey., la Gagea saxatilis Koch et plusieurs jolies Graminées. Cependant il est quelques plantes quri ne se trouvent guère ailleurs; ce sont le Glaucium corniculatum Curt. dans les cultures entre Va!ère et Tourbillon, la Genista radiata Scop. au bord de la Sionne, la Vicia lathyroides L. à Valère, l'Opuntia vulgaris Mill. sur les rochers, la Salvia officina!is $\mathrm{L}$. subspontanée, l'Iris vivesceus D C., la Tulipa Didieri Jord.

Depuis Sion, il est bon d'aller jusqu'à Bramois et a l'ermilage de Longe Borgne qui se trouve à l'entrée de la vallée d'Herens. Le long de la route lu retrouveras les plantes déjà signalées, mais c'est an bord du chemin près de l'Ermitage que j'ai cueillis les plus beaux échantillons du Clypeola Jonthlaspi L. Même j'ai vu une fois tomber d'un arbre un fruit assez singulier; c'étail un malheureux faucheur qui avait glissé sur les pâlurages abruptes qui dominent le chemin, élait tombé sur un érable qui avait amorli sa chule mais sans pouvoir l'empêcher de rebondir sur la pente et de rouler de là jusqu'au bord de la Borgne. Il en fut heureusement quitte pour la peur et se secouant comme. 
un caniche qui sort de l'eau, il s'en retourna tranquillement chez lui.

A Sion profite de la voie ferrée pour gagner St-Le 0nard, car le long de la route tu ne trouverais guère que le Lepidium ruderale L., le Sisymbium Sophia L., l'Isatis tinctoria L., le Podospermum laciniatum D C. él autres déjả récoltées.

A St-Leonard mème tu ne trouveras pas grand chose de nouveau car c'est surtout une station pour lcs plantes du printemps que je t'ai signalées dans une lettre précédente. Ciependant dirige-toi vers Sierre en passant par les collines en grande partie composées de Gypse qui s'élendent jusqu'au-delà de Granges. Peutêtre trouveras-tu le Glaucium corniculatum Curt. dans les cultures qui n'ont pas encore été envahirs par la vigne, lu chercheras la nouvelle localité du Ranunculus gramineus L. et du Bulbocodium vernum L. découvertes par l'infatigable M. Nurel; mais les deux plantes qui abondent sur ces collines sont l'Artemisia valesiaca All. et l'Onosma stellulalum W. et K.

Sierre est une pelile ville siluée dans une exposilion qui la met à l'abri du courant d'air qui balaie continuellement la vallée du Rhône surtout de Martigny à Sion; aussi je ne saurais trop la recommander, comme station dhiver, aux personnes qui veulent se mellre à l'abri de nos bises froides et désagréables, si pénibles à supporter. Probablement, comme médecin, tu conseilleras aux malades pour lesquels un changement de climal est indispensable, de passer l'hiver à Madère, au Caire, en Algérie, en Ilalie et dans le Midi de la France. Mais que de fois des gens délicats ou maladifs qui vont à Rome, à Naples, à Florence, et sur le littoral de la Méditerranée se plaignent du froid, surlout ces dcrniers hivers, el de la difficulté de se loger dans 
des appartements convenablement chaufés. Beaucoup trouveront à Veytaux, à Bex, à Sion et à Sierre un climat plus doux que celui de Genève ou de Lausanne, pas de bises, moins de brouillards et de neige et surtout des chambres bien fermées et munies de bons moyens de chauffage. A Sierre, la pension Beeguer située dans une bonne exposition, avec sa façade principale en plein soleil peut être recommandée en loute conscience. La seule objection que l'on fasse, c'est le manque d'agréments; mais un certain nombre de personnes qui s'arrangeraient pour y élablir une colonie d'hiver trouveraient par le fait de leur association suffisamment de ressources pour passer leur temps assez agréablement. Il y a d'alleurs à Sierre une quantilé de promenades aussi variées que pittoresques et quelques semaines sont bien vile passées. Si tu ajoutes à ces considérations, la dislance peu éloignée, la facilité du voyage et les dépenses relativement modérées, tu reconnaitras que tous !es avantages sont en faveur de la localilé que je te recommande. Seulement on n'y songe pas, on va oú l'habitude, la routine et l'esprit moutonnier vous dirizent et l'on délaisse pour des pays éloignés. ce que l'on a pour ainsi dire sous la main.

A Sierre l'ancien grand glacier du Rhòne a dû faire dans sa relraile une station prolongée, si l'on en juge par l'aspect singulier de la forêt de. Finges qui présente, au milieu d'un entassement confus de monticulrs, des méandres el des labyrinthes inextricables et par toutes ces collines arrondies, remaniées et rongées par le Rhône, dont les restes couvrent la vallée comme d'immenses tumulus de Géants.

Tu trouveras dans les environs immédiats de la ville, dans le bois de Finges et surtout in poussant 
jusqu'i Varone bon nombre de plintes intéressantes telles que l'Erysimum Helvelicum D C., la Buffonia macrosperma Gay., l'Ononis Columnæ All., la Coronilla coronata Gaud., le Colutea arborescens L. le Telephium Imperati L., la Rubia tinclorum L., la Pirnpinella nigra Koch., le Chrysocoma Linosyris L., l'Artemisia Valesiaca All., l'Achillea tomentosa L., l'A selacea W. et K., l'A. nobilis L., la Crupina vulgaris Class., la Centaurea Valesiaca Jord., le Xeranthemum inapcrtum Willd., la Lacluca virosa L., la L. Scariola I., la L. Augustana All., la L. perennis L., la L. viminea Sch., la L: muralis Fres., le Hieracium lanatum Vill., le H. pictum Schl, le II. Valesiacum Fries., l'Onosma :tellulatum W. et K., l'Euphrasia lutea L., l'E. visrora L. (rare), la Salvia sclarea L., l'Euphorbia lierardiana L., le Sclerochloa dura P. B., le Poa concina Gaud., le Cynosurus echinalus L., elc., elc.

Maintenant ma tàche est finie et je te dis adieu, en espérant que l'àge cl les infirmilés ne me condamneront pas à une inaction forcéc en m'empêchant de laccompagner dans quelques-unes de ces excursions, dont j'ai eu tant de bonheur à te retracer les souvenirs.

Ch. F. d. m. 


\section{ÉNUMÉRATION DES PLANTES}

les plus intéressantes de la plaine du Bas-Valais.

\section{Ranunculaceæ. - Renonculacées}

Clematis recta L. Clematite droite. Saillon, Vetroz. Sion Juillet.

Thalictrum majus Gaud. Pigamon élevé. Prairies entre Sierre et Varone. Eté.

Th. pubescens Schl. P. pubescent. Taillis près des Folateires. Juin, juillet.

Th. fœtidum L. P. fétide. Branson, Sion (Rapin) Elé. Th. anğustifolium L. P. à feuil!es étroites. Prairies humides vers Charrat. Juin, juillet.

Th. flavum L. P. jaune. Marais près de la gare de Vouvry (Reuler). Juillel, août.

Anemone Pulsatilla L. var. nutans Gaud. Anemone Pulsatille penchée. (A. monlana Hopp. Koch) Martigny, Branson, Fouly, Montorge. Sion, St-Léonard, Sierre. Avril et juin.

Hepatica triloba Chaix. Hepatique à trois lobes. (Anemone Hepalica L.) La variété à fleurs blanches et roses est plus rare el se trouve dans le bois noir près de $\mathrm{S}^{\mathrm{t}}$-Maurice. Nars, avril.

Adonis autumnalis L.) Adonide d'automne, Goutte-deSang. Plante erratique des moissons du Bas-Valais. Juin, juillet.

A. aestivalis L. A. d'été (A. ambigua Gaud), moissons à Branson, Ardon, Sion, Sierre. Mai, juin.

A. flammaea Jacq. A. rouge (A. æstivalis Gaud.) Champs maigres el gravcleux à Branson, Montorge, St-Léonard, Sierre. Mai, juin. 
A. vernalis L. A. du Printemps. Folateires, Charrat, Saxon, etc. Avril, mai.

Ranunculus trichophyllus Chaix $\beta$ Rioni. Renoncule de Rion. Etang de Montorge. Avril, juin.

R. aquatilis L. var. heterophyllus. Boveret. (D'Angreville). Mai, juin.

R. gramineus L. R. graminée. Colline de St-Léonard, Platrières de Sierre. Avril, mai.

R. replans L. R. radicante. Indiquée par Wurith au Guerset, bord dı Rhône. Mai, juin.

R. acris L. var. Boreanus Rap. R. de Boreau. (R. Boreanus Jord.) commun dans les prairies du Bas-Valais où il parait remplacer le type. Mai, juillet.

R. sceleratus L. R. scélérate. Elang de Montorge. Mai, juin.

R. nemorosus D C. R. des hois. Environs de Monthey. Mai, juillet.

R. philonotis Retz. R. des marécages. Près de Martigny. Mai, août.

Nymphaeaceae. Nympheacées.

Nuphar luteum L. Nuphar jaune. Canaux et marais. Juin, août.

\section{Papaveraceae. Papaveracées.}

Glancium luteum Scop. Glancière jaune, (G. flavum Crantz. Chelidonium Glaucium L.) Indiqué an bord des Etangs de Montorge par Murith et Gaudin, mais je l'ai cherché inutilement. Juin, noût.

G. corniculatim Gurt. Gl. violette (G. violaceum Juss.) Cultures à Montorge, Valère, St-Lóonard. Mai, juin.

Papaver argemone L. Pavot argémone. Charrat, StPierre, Sion. Mai, juin. 


\section{Fumariaceae. Fumariacées.}

Corydalis solida Sm. $\beta$. australis Haussm. Reut. Corydale bulbense australe. Gueuroz, Branson oủ elle a été découverte par II. Reuter. Mar's, avril. Elle est. indiquée par M. Rapin à Gueuroz comme variété à fleurs blanches.

\section{Gruciferae. Grucifères.}

Eruca saliva Lam. Roquelte cultivée. (Brassica Eruca L.) St-Maurice, Branson, Montorge, St-Léonard, Sierre. Mai, juillet.

Sinapis alba L. indiqué à Massongex par M. d'Angreville. Juin, juillet.

Cheiranthus Cheiri L. Giroflée jaune. St-Maurice, Sion, sur les rochers. Mai, juin.

Erysirnum Helvelicum D G. Velar suisse. Coleaux à Branson, Fonly, Contey, Montorge, Sion, St-Léonard, Sierre, Varonne, etc. Mai, juillet.

E. virgatum Roth. V. effilé. Entre Charral et Saxon le long des haies. Juin, juillet.

E. cheiranthoides L. V. fausse Giroflée. Saint-Maurice (Rapin). Mai, octohre.

Sisymbrium pannonicum Jacq. Sisymbre de Hongrie. (S. Sinapistrum Crantz) Près d'Iserabloz. Mai, juin. S. Sophia L. S. Sophie. Commun à Martigny, Sion, Sierre, etc. Mai, juin.

S. austriacum Jacq. S. d'Autriche. Longeborgne, Sion (Muret d après Gremli). Mai, juillel.

Turritis glabra L. Tourette glabre. Branson, Fouly, Sion, Sierre, etc. Mai, juillet.

Arabis saxatilis All. Arabetle des rochers. Pied du Mont-Otlan à Martigny, au-dessus de St-Léonard. Mai, juin.

A. auriculata Lam. A. auriculée. Branson. Avril, mai. 
A. sagillala D G. A. sagitlée. Vouvry, Porte du Scex. Mai, juin.

A. muralis Bert. A. des murs. St.Maurice, Rochers dı Trient. Avril, juin.

A. alpina L. A. des Alpes. Porte du Scex, Rochers du Trienı. Mai, juin.

A. Turrita L. A. Tourelle. Porte du Scex, St-Maurice, Marligny. Mai, juin.

Cardamine impatiens L. Cardamine impatient. Indiquée par Murith à Martigny. Mai, juillet.

Dentaria digitata Lam. Dentaire digitée, dans les bois sous Létroz (Gueuroz). Mai, juin.

D. pinnala Lam. D. ailée. Vouvry. Mai, juin.

Lunaria rediviva L. Lunière vivace. Porte du Scex, Vouvry (Gaud. Murith). Mai, juin.

L. biennis Moench. L. bisanuelle. Cultivée dans les jardins, a élé trouvée en abondance aux envirous de Marligny après l'inondation de la Dranse, mais on ne l'a pas revue. Mai.

Clypeola Jonthlaspi L. Clypeole Jonthlaspi. Saillon, Tourbillon et Valère à Sion, près de l'ermitage de Longeborgne. Avril, mai.

Draba muralis L. Drave des murs. Indiquée par Murith et Caudin au pied du Mont-0ttan avant d'entrer à Martigny et ì St-Naurice; j’ai exploré vainement ces localités à plusieurs reprises mais j’ai retrouvé la plante en quantité le long des haies entre Martigny el Charrat par l'ancienne route. Mai.

D. aizoides L. D. aizoide. Rochers de la Porte de Scex, du Trient. Avril, juin.

Vesicaria utriculata Lam. Vesicaire enflée. (Alyssum utriculatum L.) Rochers près de la Cascade de Pissevache et du Trient. Environs de Martigny. Avril, mai. 
Cochlearia saxalilis Lam. Cranson des roches (Myagrum saxatile L.) Rochers du Trient. Mai, juillet.

Camelina sylvestris Wallr. Cameline sauvage (C. microcarpa Andrz.) Champs du Bas-Valais, Martigny, Branson, Charrat, Montorge, St-Liconard, etc. A vril, juin.

C. dentata Pers. C. dentée. Marligny, Branson. Mai, juin.

Calepina Corvini Desv. Calepine faux-cranson. (Bunias cochlearioides Murr.) Champs de Branson, St-Maurice (Schleich.) Plante erratique et capricieuse. Mai, juin.

Neslia paniculata Desv. Neslie paniculée. (Myagrum paniculatum L.) Champs à Vetroz. Mai, juillet.

Bunias Eracago L, Bunias fausse-Roquetle. Montorge. juin, juillet.

Myagrum perfoliatum L. Myagre perfolié. Indiqué par Murith dans les champs de Vetroz. Juin, juillet.

Lepidium graminifolium L. Passerage à feuilles de Gramen. Sion, Saillon, Contey, (Gaud. et Murith). Juin, août.

L. Draba L. P. Drave. Trouvé près de Sion, par M. Haussknechı, n'est pas indiqué par Murilh ni par Gaudin. Juin, juillet.

L. petracum L. P. des rocailles. Porle du Scex, Martigny, Branson, Saxon, Sion, elc... Avril, mai.

L. Alpinum '.. P. des Alpes, se trouve quelquefois au pied de la cascade de Pissevache, dans les débris descendus des hauteurs. Juin, juillet.

Thlaspi ruderale All. Tabourel des décombres (Lepidium ruderale L.) Le long des roules et des murs, Martigny, Sion, etc. Mai, août.

Biscutella lævigata L. Lunetière lisse. Sur les rochers de Saint-Maurice à Martigny, surtout au Trient, 
aux Folatcires, mélangé avec la var. $\beta$. saxatilis Rap. (B. saxatilis Schl. Gaud.) Celte variété se distingue du type par ses siliques pointillées de petites élévations blanchâlres : Mai, juin.

Ethionema saxatile R. Br. Ethionème des Rochers. (Thlaspi saxatile L.) indiqué par Murith à Contey. Mai, juin. Je ne crois pas qu'il ait été trouvé par les bolanistes modernes.

Isatis tinctoria L. Pastel des teinturiers. Branson, Ardon, Leytron, Montorge, Sion, etc. Avril, juillet.

Senebiera Coronopus Poir. Senebière Corne-de-cerf (Coronopus Ruellii All. Gaud.) indiqué à St-Maurice par Murith. Juin, août.

\section{Gistineae. Gistinées.}

Ilelianthemum salicifolium Pers. Helianthème à fenilles de saule. Branson au bord dı sentier conduisant aux Folateires. Avril, mai.

H. Fumana Mill. (Cistus fumana L.) Fouly, Saillon, Sion. Mai, juin.

\section{Violarieae. Vio!ariées.}

Viola sciaphila. Koch, au-dessus de Branson en montant à Joux-Brûlée (Rapin), Montorge, Tourbillon (Hausknecht). Mai, juin.

V. arenaria, D C. V. des sables. Pelouses des Folateires. Avril, mai.

V. mîrabilis L. V. singulière. Porte du Scex, où je l'ai trouvée en assez grande quantité au pied des rochers, au-dessus de Branson, en montant à Joux-Brûlée (Rapin), près de Riddes (Murith). Avril, mai.

Obs. Sous le nom de Viola Ruppii All. Murilh indique comme rare une violelle qui habite les marais près de Martigny. Gaudin la décrit sous le nom de V. Montana III Ruppii. Il est probable que l'on trouvera dans 
les localités marécageuses du Bas-Valais les V. stagnina Kit. et V. pumila Vill.

V. Stevinii Bess. Indiquée à Valère (Sion) par M. Ilausknecht. Avril, mai.

V. segetalis Jord. V. des moissons (V. tricolor L. var.) Branson: Bull. soc. Mur. Mai, juillet.

V. tricolor L. var. minima, Gaud. V. tricolore naine. (V. arvensis Murr. Bcanescens Rapin). Branson, Fouly, Sion, St-Léonard. Avril, mai.

\section{Droseraceae. Droséracées.}

Drosera rotundifolia L. Rossolis à feuilles rondes. Petit marais de Salvan. Juin, juillet (Murith).

\section{Garyophylleae. Garyophyllées.}

Silene noctiflora L. Silène de nuit. Martigny. (Murith, Gaudin). Juin, seplembre.

S. Armeria L. S. Arméria. Salvan, Branson, Folateires, Fouly. Juin, juillet.

S. rupestris L. S. des rochers. Gueuroz. Juin, juillet.

S. Otites Sm. S. à petites fleurs. Martigny, Branson, Fouly. Mai, juillet.

Lychnis viscaria L. Ly shnide visqueuse. Val de Salvan (Mercier). de l'ai tronvée à Gueuroz. Mai, juin.

L. Coronaria Lam. L. des jardins. (Agrostemma Coronaria L.), au-dessus de Branson el de Fouly. Juin, juillet.

L. Flos-Jovis Desr. L. Fleur de Jupiler (Agrostemma Flos Jovis L.) Mayens de Fouly (Murith).

Buffonia macrosperma Gay. Buffonie à grosses graines (B. tenuifolia Gaud. Rapin non L. B. annua Murith), Charrat, Ardon, la Morge (Muret), Sion, Sierre, Salgetsch, Varonne. Je l'ai trouvée en quantité et en supurbes érhantillons à Sierre avec M. Rapin. Juillel, septembre. 
Alsine fasciculata M. et K. Alsine fasciculée. Martigny, Charrat, Sion, Sierre. Juin, août.

Holosteum umbellatum L. Holostée en ombelle. Bord des haies, Marligny, Branson, Charral, etc. Avril, mai.

Cerastium arvense L. var. $\alpha$. Rap. Céraiste des champs. Martigny, Branson. Mai, juin.

\section{Geraniaceae. Geraniacées.}

Geranium palustre L. Géranium des marais. Marais près de la gare de Vouvry (Reuter). Juillet août.

G. sanguineum L. G. sanguin. Martigny, les Marques. Mai, aoùt.

G. lucidum L. G. luisant. Je l'ai récolté au pied des rochers entre Pissevache et Marligny. Avril, juin.

Balsamineae. Balsaminées.

Impatiens noli-langere L. Impaliente commune. La Combe, Martigny (Ilurith). Juin, juillet.

\section{Rutaceae. Rutacées.}

Ruta graveolens L. Rue odorante. Rochers de St-Naurice en montant à l'ermilage. Varonne. Juin, Août.

\section{Terebinthaceae. Terebenthacées.}

Rhus cotinus L. Sumac Fustet, Arbre à perruque. Colline des Vlarques, entre Louesche et Campel (Murilh). Mai, juillet.

\section{Rhamneae. Rhamnées.}

Rhamnus alpinus L. Nerprun des Alpes. Rochers de St-Maurice vers l'ermitage. Mai, juin.

R. pumilus L. N. nain. Rochers de Valère et de Tourbillon à Sicn (Murith, Gaudin). Mai, juin.

\section{Leguminosae. Légumineuses.}

Genista radiata Scop. Genêl rayonnant. Au dessus d'Ardon (Gaudin). Entre Ardon et la station de che- 
min de fer. Ravin de la Sionne près du moulin audessus de Sion, où nous l'avons récolté avec M. Rapin d'après les indications de MM. Leresche et Muret. Juin, juillet.

Ononis altissima Lam., Murith, Bugrane élevée $(0$. hircina Jacq. Gaud. 0. fælens All.) près de Martigny, prairies entre Saxon el Charrat. Juin, juillet.

0 . Columnæ All. B. à petites fleurs (0. parviflora Lam. D G.) Marligny, Fouly, Saillon, Montorge, Sion, StLéonard, Sierre, etc. Mai, juillet.

0. Natrix L. B. gluante. Martigny, Branson. Juin, août.

O. rolundifolia L. B. à fevilles rondes. Saxon. Mai, juille..

Trigonella Monspeliaca L. Trigonelle de Montpellier, Branson, Fouly, Saillon, Montorge, Sion. Avril, juin. Trifolium cœspitosum Reyn. Trèfle gazonnant. Indiqué par M. Rapin à St-Maurice. Juin, août.

Colutea arborescens L. Baguenaudier commun. SaintMaurice, Fouly, Sierre, elc. Juin, juillet.

Oxytropis Halleri Bung. Oxytropis de Haller. (Oxytropis Uralensis Gaud. non DC. (Astragalus Uralensis Sut. Nurith, non L.) Aux Folateires, à Charrat, à Saxon et à Sierre. Avril, Juin.

0. campestris DC. 0. champêtre. (A. campestris L. Sut. Mur.) Martigny, la Bàtia. Juin, juillet.

O. pilosa DC. O. poilue. (A. pilosus L. Sut. Mur,) Martigny, Folateires, Charral, Riddes etc. Mai, juillet.

Astragalus Onobrychis L. Astragale Esparcette. Martigny, Branson, Saxon, Charrat etc. Mai, juillet.

A. Cicer. L. A. Pois-chiche. Martigny, Branson, Saxon. Juin, août. 
A. Monspessulanus L. A. de Montpellier. Saxon, Montorge, Saint-Léonard, Sierre. Mai, juillet.

Coronilla coronata DC. Gand. non L. Coronille couronnée. (C. minima L. non D C. C. montana Murith non Scop. G minima L. $\beta$ loloides Koch.) Je ne l'ai rencontrée qre près de Varonne. Juin, seplembre.

Onobrychis arenaria D G. Esparcetle des sables. (0. supina Gaud). Folateires, Saxon, Montorge, St-Léonard, Sierre. Juin, août.

Vicia pisiformis L. Vesce à feuilles de Pois. Bois audessus de Fouly. Mai, juillet.

V. sylvatica L. V. des bois. Au-dessus de Fouly avec la précédente. Mai, juillet.

V. dumelorum L. V. des buissons. Avec les précédentes: Mai, juillet.

V. tenuifolia Roth. V. à feuilles menues. (V. Cracca L. III. tenuifolia Gaud.) Marligny, Branson, Fouly, Saxon, Charrat. Mai, juillet.

V. Gerardi D C. V. de Gérard. (V. Gracca L. II Gerardi Gaud.) Fouly, Saxon, Charrat. Mai, juillet.

V. onobrychioides L. V. fausse-Esparcette. Branson, Fouly, Sion, Sierre. Mai, juillet.

V. lathyroides L. V. fausse-Gesse. Sion entre Valère et Tourbillon. Avril, mai.

Orobus niger L. Orobe noir. Branson, Fouly. Mai, juin. Lathyrus sphœricus Retz. Gesse sphérique. Branson. Mai, juin. C'est bien cette espèce qui croît en Valais et non pas le $\mathrm{L}$. angulatus $\mathrm{L}$. indiqué à tort à Branson el à Martigny par Murith et Gaudin.

L. Luberosus L. G. Lubéreuse. Martigny dans les champs. (Murith). Juin, juillet.

L. palustris L. G. des marais. Bouveret, elc. Juin, août. 


\section{ร7}

\section{Rosaceae. Rosacées.}

Amgydalus communis L. Amandier commun. Saillon, St-Léonard, Sierre. Février, avri!.

Cerasus Mahaleb D C. Cerisier Mahaleb. Bois de SainteLucie. Entre Nartigny et Charrat. Mai, juin.

Rubus glandulosus Bell. Ronce glanduleuse. Marligny. (Bull. de la soc. Mur.) Juin, juillet.

R. collinus D C. R. des collines. Au-dessus de Branson (Rapin). Juin, aoùt.

R. tomentosus Will. R. tomenteuse. Indiqué à Martigny par Murith. Juin, juillet.

Fragaria collina Ehrh. Fraisier des collines. Indiqué à Branson par Gaudin. Mai, juin.

Comarum palustre L. Comaret des marais. Sous Fouly. Juin, juillet.

Potentilla caulescens L. Potentille caulescente. Rochers de la Porte du Scex, St-Maurice. Juin, août.

P. rupestris L. P. des rochers. Entre Salvan et Gueuroz, la Croix, près de Martignny. Mai, juin.

P. verna L. var. cinerea Rap. P. printanière cendrée (P. cinerea Gaud. non Chaix) Martigny, Branson, Fouly, Si. Léonard. Avril, mai.

P. recla L. P. droite. Les Marques, Branson. Mai, juillet.

P. parviflora Gaud. P. à pelites fleurs. Martigny, les Marques. (Mirith). Mai, juillet.

P. inclinata Will. P. inclinée. Indiquée par Murith a Branson; Irouvée par M. Hausknecht, au-dessus de Marques. Mai, juin.

Spiræa Filipendula L. Spirée filipendule. Saxon, Contey. Mai, juin.

Rosa cinnamomea L. Rosier canelle. Martigny et StBranchier (Murith). Mai, juillet. 
Mespilus germanica R. Néflier commun. Mont-Chemin. (Murith). Mai, juin.

Cotoneaster vulgaris Lindl. Colonnier commun (Mespilus Coloneaster L.) Martigny.

C. tomentosa Lindl. C. tomenteux. Sion, Tourbillon. Mai, juillet.

Cydonia vulgaris Pers. Coignassier commun (Pyrus Cydonia L.) Coa et là dans les haies du Bas-Valais. Mai, juillet.

Sorbus hybrida L. Sorbier hybride. St-Maurice (Thomas, Gaudin): Mai, juin.

Amelanchier vulgaris Miench. Amélanchier commun. (Mespilus Amelanchier L.) Colline des Marques (Murith). Avril, juin.

R. Gallica L. R. de France. Ardon, Vetroz, environs de Sion. Mai, juin.

R. monlana Chaix. R. de montagne (R. rubrifolia Vill. var. montana Gaud. R. glandulosa Bell.) Salvan (Murith) au-dessus de Branson, à Montorge (Rapin). Juin, juillet.

\section{Onagrarieae. Onagrariées.}

Enolhera biennis L. Enothère bisannuelle. Vouvry. Juillet, août.

Granateae. Granatées.

I'unica Granalum L. Grenadier commun. Sion, rochers de Valère (Murith). Juillet.

\section{Philadelpheae. Philadelphées.}

Philadelphus coronarius L. Seringat odorant. Près de de Martigny et de Sion (Murith) échappé de jardins. Mai, juin.

\section{Gucurbitaceae. Gucurbitacées.}

Bryonia alba L. Bryone blanche. Plante nouvelle pour le Valais où elle n'avait jamais élẻ trouvée. D'après 
Gremli (supplément) M. Muret l'a découverte près de Saxon, Tourtemagne et Viége. Juin, juillet.

\section{Paronychieae. Paronychiées.}

Telephium Imperati L. Télèphe à feuilles alternes ( $T$. alternifolium Clairv.) Saillon, Contey, Sion, St-Léonard. Mai, juillet.

Herniaria glabra L. Herniaire glabre. Gueuroz, Martigny, Branson, Charrat, St-Léonard. Mai, septembre.

\section{Sclerantheae. Scleranthées.}

Scleranthus verticillatus Reich. Gnavelle verticillée Gueuroz, Branson, Sion, St-Léonard. Avril, mai.

S. perennis L. G. vivace. Branson, Martigny. Mai, juillet.

\section{Grassulaceae. Crassulacées.}

Sedum maximum Sut. Orpin élevé, Reprise (S. Telephium $\beta$ L. Martigny, aux Marques, Juin, août.

S. dasyphyllum L. 0. à feuilles épaisses. Ça et là sur les murs dans le Bas-Valais (Murith.) Juin, août.

S. cepaea L. 0. paniculé. St-Gingolph. Juin.

S. sexangulare L. 0. sexangulaire. Folateires. Juin, juillet.

Sempervivum tectorum L. Joubarbe des toits, Porte du Scex. Marligny, Branson, Charral, Sion, Sierre, el . Juin, août. On en rencontre plusieur's formes qui mériteraient d'être mieux éludiées.

S. Arachnoideum L. à toiles d'araignée. Rochers du Trient, la Bàtia, Branson, Charral, Sion, Sierre, C'est la forme qui, je crois, a été appelée S. tomentosum. Mai, juin.

\section{Saxifrageae. Saxifragées.}

Saxifraga oppositifolia L. Saxifrage à feuilles opposées. 
Espèce alpine descendue dans Bois-Noir depuis la Dent du Midi. Avril, mai.

S. exarata Vill. Rap. var. leucatha. S. gazonnante. (S. cæspitosa III leucantha Gaud.) Rocbers du Trient en montant à Gueuroz, Branson, Fouly. Mai, juin.

S. bulbifera L. S. bulbifère. Gueuroz, Branson, Mai, juin.

S. stellaris L. S. éloilée. Rocher's de Gueuroz. Mai, juin.

S. cuneifolia L. S. cuneiforme. Rochers de Gucuroz. Mlai, juin.

Cacteae. Cactées.

Opuntia vulgaris Nill. Caclier commun, Raquelte, Figue d'Inde. (Cactus Opuntia L.) Rochers de Valère et de Tourtillon, Saint-Léonard. Juin, août.

\section{Umbelliferae. Umbellifères.}

Trinia vulgaris DC. Trinie commune (Pimpinella dioica L.) Les Marques. Mai, juin.

Pimpinella nigra Koch Boucage noire. (P. Saxifraga L. III nigra Gaud.) Marligny, les Marques; Sierre. Juillet, août.

Carum Bulbocastanum Koch. Carvi Noix-de-lerre. Martigny Fouly. Juin, juillet.

Cicula virosa L. Ciculaire vireuse. Marais sous Charral, Fouly (Nurith) Etang de Montorge (Reuter). Juin, août.

Sium latifolium L. Berle à larges feuilles. Bouveret. Juin, août.

Buplevrum rolundifolium L. Buplèvre à fenilles rondes. Champs à Montorge, Saint-Léonard. Contey. Juin, juillet.

Seseli bienne Grantz. Séseli bisannuel (S. annuum L.) 
Saint-Maurice, la Bâlia, les Folaleires. Juin, septernhre.

Trochiscanthes nodiflorus Koch. Trochisque nodiflore. Bouveret, Port-Valais, pied des rochers entre SaintMaurice et Marligny (Gandin). Jıin, aoùt.

Fœniculum officinale All. Fénouil officinal. (Anethum Fœ-L.) Vignes du Bas-Valais (Murith), Juillet, août. Peucedanum venelum Koch. Peucedanum de Venise (Cervaria alsatica $\beta$ albifiora Gaud.) Vignes de Ravoire près de Martigny, près de Pissevache (Muret). Juin, septembre.

P. Chabraei Gaud. P. de Chabrey. Ravoire de Martigny. Juillel, août.

P. Cervaria Lap. P. des cerfs (Athamatha cervaria L. Fouly. Juin, juillet.

P. Oreoselinum Mœnch. P. des mortagnes (A. Oreoselinum L.) La Bàtia, les Niarques, Fouly.

Pastinaca onaca Bernh. Panais opaque (P. sativa L. propart.) au bord des chemins, à Fouly, Sion, etc. Juillet, août.

Laserpitium Siler L. Laser Siler. St-Maurice, etc. Juin, août.

Orlaya grandiflora Lam. Orlaya à grandes fleurs (Caucalis grandiflora L.) Branson. Juin, juillel.

Caucalis daucoides L. Caucalide fausse-carolte. Champs graveleux à Branson, Montorge, St-Léonard. Juin, juillet.

Turgenia latifolia Hoffm.'Turgenie à larges feuilles. (Caucalis latifolia L.) Champs à Saillon, Contey, St-Séverin. Juin, juillet.

Anthriscus cerefolium Hoffm. $\beta$ trichosperma Rap. Anthrisque cerfeuil. Valère et Tourbillon. Mai, juin.

A. vulgaris Pers. A. commune (Scandix Anthriscus L.) Branson, Fouly, Saillon, Sion. Avril, juin. 
Conium maculalum L. Cigüe tachetée,'grande Cigüe. Fouly, Charrat, Martigny. Juin, août.

\section{Corneae. Cornées.}

Cornus mas L. Conrnouiller commun. Bois noir près de St-Murice, etc. Mars, avril.

\section{Loranthaceae. Loranthacées.}

Viscum album L. Gui commun. Sur les Pins près de la gare d'Ardon. Mars, avril.

\section{Gaprifoliaceae. Caprifoliacées.}

Lonicera Etrusca Santi. Chèvrefouille dEtrurie, Sion, Contey, entre Fouly et Saillon (Muret). Juin, juillet.

\section{Rubiaceae. Rubiacées.}

Galium Pedemontanum All. Gaillet du Piémont. Fouly, Branson. MM. Muret et Rapin en ont trouvé de magnifiques échantillons en montant à Joux-Brûlée, audessus de Branson. Mai, juin

G. tricorne With G. tricorne. Charral, Saint-Pierre. Juin.

G. Spurtum L. $\beta$ Vaillantii D C. G. de Vaillant. St-Manrice, St-Léonard. Mai, juin.

Rubia tinctorum L. Garance des teinturiers. Naturalisée à la Porte du Scex, à Contey, à Sion et à Sierre; peut-être provient-elle d'anciens essais de culture. Juillet, août.

Asperula longiflora W. et K. Koch. Asperule lâche (A. cynanchica L. $\beta$ longiflora Reich.) Mart gny, Branson, St-Léonard. etc. Juin, août.

A. tinctoria L. Asperule tinctoriale. Indiquée par Murith sur le coleau des Marques. Juin, juillel.

A. arvensis L. A. des champs. Fouly, Contey. Juin, juillet. 


\section{Valerianeae. Valérianées.}

Centranthus ruber DC. Valeriane rouge. Sion, Rocher's de Tourbillon. Mai, juillet.

\section{Compositae. Composées.}

Tussilago Petasites L. $\beta$ hybrida. Tussilage Petasite (Petasites officinalis Mœnch. $\beta$ sub-fœmineus D C.) Cette variété dont presque toutes les fleur's sont femelles, croil en abondance près de Martigny, le long des canaux de la Dranse, en suivant le chemin qui conduit à Branson. Avril, mai.

Senecio paludosus L. Senecon des marais. Celle belle plante a été tronvée par M. Reuter dans le marais de Vouvry, près de la Gare. Indiquée an Bouveret. Juin, août.

S. sylvaticus L. S. des bois. Au-dessus de Fouly. Juin, août.

Inula Britannica L. Inule aqualique. Indiquée par $\mathrm{Hu}$ rith, près de St-Léonard. Août, septembre.

I. Helenium L. I. Aunée. Martignny, Murith. Juin, juillet.

Chrysocoma Linosyris L. Chrysorome à feuilles de lin. (Linosyris vulgaris Cass. DC.) Marligny anx Marques, abondant à Sierre. Juillet, septembre.

Aster amellus L. Aster amellus. Vignoble des Marques, Sierre. Août septembre.

Obs. Le Stenactis annua Nees (Aster annuus L.) est indiqué en Valais par Gaudin, sans désignation de localité. L'Aster brumalis Nees. amrait été trouvé près de Vouvry, plus ou moins naluralisé.

Gnaphalium loteo-album L. Gnaphale jaunatre. Charrat, Bramois, entre Sion et Loueche. Juin, août.

Micropus erectus L. Micrope dressé. Montorge, StLéonard, Sierre, Varonne. Mai, juillet. 
Carpesium cernuum L. Carpesie penchée. Indiqué à Fouly par Murith. Juillet, septembre.

Artemisia Absinthium L. Armoise Absinthe, grande Absinthe. Coteaux de Branson, Montorge, St-Léonard, Sierre. Aoûl, septembre.

A. Valesiaca All. Armoise du Valais. Branson, Fouly, Saillon, Contey, St-Léonard, Sierre. Septembre, octobre.

Achillea tomentosa L. Achillée tomenteuse. Branson,

Fouly, St-Pierre, Sierre. Mai, septembre.

A. setacea W et K. A. sétacée. Branson, Charral, Saxon, Sion, etc. Juin, septembre.

A. nobilis L. A. noble. Martigny, Branson, Fouly, Montorge, Sion, St-Léonard. Juin, septembre.

Echinops sphærocephalus L. Echinope à tète ronde. Branson, Sion, Sierre. Juin, août.

Silybum Marianum Gærın. Chardon-Marie (Carduus Marianus L.) Branson. Juin, août.

Lappa pubens Bor. Bardane pubescente. (L. minor D C. $\beta$ pubens Rap.) Trouvée par M. Iaussknecht entre Vetroz et Sion. Juillet, août.

Crupina vulgaris Cass. Crupine vulgaire (Centaurea Cr'upina L.) Branson, Fouly, Montorge, Sion, St-Leonard, Sierre. Juin, août.

Xeranthenum inapertum Wild. Gaud. Immortelle fermée. (X. annuum $\beta$ L.) Nartigny, Saillon, Montorge, Sion, St-Léonard, Sierre, etc. Juin, août.

Centaurea Valesiaca Jord. Rap. Centaurée du Valais. (C. paniculata Gaud. non L.) Branson, Montorge, Sion, St-Léonard, Sierre. Juin, septembre.

C. lanata D C. C. laineuse. (Carthamus lanatus L.) Indiqué par Murith le long de la Morge et à Valère (Sion). Juin, août.

Chondrilla juncea. L. Chondrille joncière. Fréquente 
en Valais; on la trouve à Martigny, Branson, Contey, Sion, Sierre, etc. Juillet, septembre.

Lacluca virosa L. Laitue vireuse. Branson, Sierre. Juillel, août.

L. Scariola L. L. Scariole. Branson, Saxon, Sierre. Juillet, août.

L. Augustana All. Gaud. L. de la vallée d’Aoste. (L. Scariola var. integrifolia). Sicrre, Varone. Juillet, . août.

L. Saligna L. L. Saulière. Fouly, Saillon. Juillet, septembre.

L. perennis L. L. vivace. St-Maurice, Martigny, Branson. Mai, juillét.

L. viminea Sch. L. rameuse (Prenanthes viminea L. P. ramossissima Gaud. Phœnixopus vimineus Reich). Martigny, La Morge, Plâtrières de Sierre. Juillet, août.

Sonchus palustris L. Laitron des marais. Rives du Rhône. Juillet, août.

Hieracium pilosella. L. var. niveum. Epervière piloselle. Indiqué á Sion par Ducommun et dans le Bulletin de la Société Murithienne. Mai, octobre.

II. Peleterianum Mer. E. de Le Peletier. Sion, Sierre. Mai, octobre.

II. pratense Tausch. Koch. E. des prés. (H. collinum Gochn. H. cymosum Wild. H. dubium L.) Montorge, Sion (Rapin). Les Marques, St-Lécnard. (Hauss. knecht). Juin, juillet.

H. glaucum All. E. glauque. Indiquée à St.-Maurice el à la Bâtia par Murith. Juin, juillet.

II. cymosum Vill. E. ả bouquet. Martigny, les Folateires. Juin, juillet.

Obs. Le II. saxatile Vill. (H. Lawsonii Vill.) est indiqué comme espèce, dans Bas-Valais à Martigny, par 
Gremli et Ducommun. Gaudin le mentionne comme variété de son $H$. murorum I vulgatum. Je ne l'ai pas récolté.

II. lanatum Vill. E. laineuse. Rochers entre Charrat et Saxon, à Saillon, entre Varonne et Inden. Juin, juillet.

H. pictum Schl. E. mouchetée. Rochers à St-Maurice, Saxon, Sion, Sierre. Juin, juillet.

H. Jacquini Vill. E. de Jacquin. St-Maurice, au Rosei. Juin, août.

H. amplexicaule L. E. embrassante. Rochers de SaintMaurice. Juin.

H. pulmonarioides Vill. E. pulmonarioide. Trouvé par II. Haussknecht sur les rochers de St-Maurice. Juin, août.

H. Valesiacum Fries. E. du Valais. Entre Sierre et Varonne. Juillet, septembre.

Tragopogon major Jacq. Salsifis majeur. Marligny, Branson, Charrat, Sion, Longeborgne, Sierre, elc. Mai, juin,

Scorzonera Austriaca Willd. Scorzonère d'Autriche. St-Maurice, Branson, Saxon, Montorge, St-Léonard. Mai, juin.

Podospermum, laciniatum D C. Podosperme lacinié n'est pas rare dans le Bas-Valais, Branson, Sion, Longeborgne, Sierre, elc. Ma i, juillel.

\section{Ambrosiaceae. Ambrosiacées.}

Xanthium strumarium L. Lampourde commune. Martigny, Branson, Sion, St-Léonard, Juin, septembre.

\section{Campanulaceae. Campanulacées.}

Campanula Bononiensis L. Campanule de Bologne. SaintBrancher, Fouly, Contey, Aven. Juillet, août.

C. spicata L. C. en épi. Environs de Martigny, la Bâtia, les Marques, Fouly, Montorge. Juin, juillet. 


\section{Ericineae. Ericinées.}

Erica carnea L. Bruyère incarnate. Abondante au Bois noir, près de St-Maurice. Mars, avril..

\section{PYROLAGERE. PYROLAGÉES.}

Pyrola arenaria Rap. Pyrole des Sables. (P. rotundifolia var. arenaria Koch. P. media Thom. non $\mathrm{S} w$. Vallée de Bagnes. Juillet.

\section{Apocineae. Apocinées.}

Vinca major L. Pervenche à grandes fleurs. Sion près du collége (Murith). Avril, mai.

\section{Gentianeae. Gentianées.}

Menyanthes trifoliata L. Trèfle de marais. Au bord du Rhône, au-dessous Fouly. Mai, juin.

Chlora perfoliata L. Chlore perfoliée. Vernayaz, PrazFalcon, entre St-Léonard et Sierre. Juin, août.

Gentiana Pneumonanthe L. Gentiane à feuilles étroites. Lens près de Sierre. Juillel, septembre.

G. verna L. G. printanière. Descend dans la plaine dans plusieurs localités, au-dessus de Vouvry, etc. Mars, avril.

G. germanica Willd. G. d'Allemagne (G. amarella Gaud.) Près de la cascade de Pissevache. Août septembre. Erythraea pulchella Fries. Erỵthrée élégante. Plâtrières de Sierre. Juillet, septembre.

\section{Boragineae. Boraginées.}

Onosma stellulatum W. et K. Orcanelte étoilée. ( 0 . montanum Gaud. 0. Echioides L. $\gamma$ Helvetica Prodr. in nota. 0. Helvetica Boiss. Diagn.) Les Marques, Branson, Fouly, Sion, Sierre. Juin, septembre. Lithospermum purpureo-cœruleum L. Gremil violet. Monthey, Branson, Mai, juin. 
Heliotropium Europaeum L. Heliotrope d'Europe. Branson, les Marques, Sierre. Juin, septembre.

Myosolis stricta Linck Myosotis roide. Branson, se trow vera probablement ailleurs. Avril, mai.

M. hispida Schl. M. des collines (M. collina Ehr. Gaud.) Coleaux arides, Branson, Montorge, etc. Avril, mai.

Obs. Le M. versicolor Pers. eṣt indiqué dans les champs, sans localités.

Echinospermum Lappula Lehm. Echinosperme Bardane (Myosolis Lappula L.) St-Léonard, entre Sierre et Varonne. Juin, août.

Asperugo procumbens L. Rapette couchée. Martigny, Branson. Mai, juillet.

\section{Solaneae. Solanées.}

Physalis Alkekengi L. Coqueret Alkekenge. Martigny, Fouly, Contey, Varone. Juin, août.

Datura Stramonium. L. Datura Stramoine, Pomme épineuse. Martigny, Sion. Juillet, août.

\section{Verbasceae. Verbascées.}

Verbascum thapsiforme Schr. var. bicolle Gaud. Molène thapsiforme, Bonhomme. St-Maurice, Martigny, Branson. Juin, août.

V. montanum Schr. M. des montagnes (V. crassifolium Gaud. D G.) Martigny, Nontorge, Sion, Juin, août.

V. incanum Gaud. M. incane. Au-dessus de Branson. Juin, août.

\section{Scrophulariaceae. Scrofulariacées.}

Scrophularia vernalis L. Scrofulaire printanière. Indiquée par Murith et Gaudin, au-dessus de Martigny, au pied du Mont-Ghemin. Mai, juin. 
Erinus alpinus L. Erine des Alpes. Gravier de la Cascade de Pissevache. Mai, juillet.

Limosella aquatica L. Limoselle aquatique. Bords du Rhône, entre Lavey el Branson (Iluret d'après Rapin) Juillel, octobre.

Antirrhinum majus L. Muflier à grandes fleurs. Murs de Sion. Juin, septembre.

Linaria cymbalaria L. Linaire cymbalaire. Vieux murs, Sion, etc. Mai, Octobre.

Picularis palustris L. Pediculaire des marais. Vouvry, Vernaya, Marligny, Fouly. Mai, juillet.

Euphrasia lutea L. Euphraise jaune. Martigny, Sierre, Varonne. Août, septembre.

E. viscosa L. E. visqueuse. Sierre, forêt de Finges, audessus de Varonne. Août, septembre.

Veronica prostrata L. Véronique couchée. St-Vlaurice. Martigny, Branson, Charrat, Sicre, elc. Mai, juin. V. spicata Koch. $\beta$ persicifolia Gaud. Véronique en épi. Les Marques. Juin, juillet.

V. fruticulosa L. V. fruticuleuse. Gueuroz. Juin, août.

V. Acinifolia L. V. à feuilles d'Acinos. Branson, Charrat, Contey. Avril, mai.

V. verna L. V. printanière. Branson, Montorge, Sion, Longe borgne, St-Léonard etc. Avril, Mai.

V. tryphyllos L. V. à trois lobes. Marligny, Branson, St-Léonard. Avril, mai.

V. præcox. All. V. précoce. Branson, Leytron, Charrat, Ardon, Sierre.

\section{Orobancheae. Orobanchées.}

Orobanche Artemisiæ campestris Vauch. Orobanche de l'Armoise. Branson, Montorge. Juin, juillet.

0. Scabiosæ Koch. 0. de la Scabieuse. Trouvée à Marligny par M. Haussknecht. Juin. 
Phelipæa cœrulea Mey. Phelipée bleue. Branson, Montorge. Juin, juillet.

P. arenaria Walp. P. des sables (Orobanche cœrulea Gaud.) Les Marques, Branson, Montorge. Juin, juillel.

P. ramosa Mey. P. du chanvre. Çà et là dans les cultures de chanvre. Juillet, septembre.

Lalhræa squamaria L. Lathrée écailleuse. Vers Pissevache, Leytron. Avril, mai.

\section{Labiatae. Labiées.}

Hyssopus offcinalis L. Hysope officinal. Marligny, Branson. Juin, août.

Origanum vulgare L. II prismalicum Gaud. Origan prismatique (0. creticum L. Sul. Ileg.) Plus rare que la forme ordinaire. Martigny, Sierre, Varone. Juillet, seplembre.

Thymus Serpyllum L. $\vee$ prannonicus Rap. Thym serpollet lanugineux. (T. pannonicus All. T. lanuginosus Schl.) Branson, Fouly, Contey, etc.. Juin, septembre. Calamintha nepetoides Jord. Calement faux-nepeta. (C. nepeta Gaud). Ardon (Hausslinecht). Juillel, septembre. Rosmarinus officinalis L. Romarin officinal. Sion, sur les rochers de Tourbillon et de Valère. Avril, juin. Melissa officinalis L. Mélisse officinale. Marti ny, Fouly, Riddes. Juin, août.

Salvia officinalis L. Sauge officinale. Naturalisée sur les rochers, à Fouly, Valère et Tourbillon. Juin, juillet.

S. verticillata L.S. verticillée. Vouvry, val d'Illier. Juin, septembre.

S. sclarea L. S. sclarée. Fouly, Contey, Sierre. Juin, août.

S. glutinosa L. S. glutineuse. Martigny. Juillet, septembre. 
Nepeta Cataria L. Nepeta Chataire. Martigny, Fouly, Sion, elc. Juin, septembre.

Dracocephalum austriacum L. Dracncéphale d'Autriche. Retrouvé par M. Nuret sur des rochers près de Dorénaz; mais il parait que la localité a été détruite. Ilai, juin.

Lamium incisum Willd. Lamier incisé. St-Maurice, Marligny. Avril, octobre.

L. album L. L. blanc. Martigny. Mai, octobre.

Stachis Germanica L. Epiaire d'Allemagne. Montorge, Sion. Juin, août.

Narrubium vulgare L. Marrube commun. Branson, Fouly, Saillon, Sion. Juin, septembre.

\section{Lentibularieae. Lentibulariées.}

Utricularia vulgaris L. Utriculaire commune. Marais. Çà et là près de Mlartigny, etc. Juin, août.

U. yninor. L. U. fluette. Moins fréquente, mêmes localités. Juin, août.

\section{Primulaceae. Primulacées.}

Primula variabilis Goup. (P. acauli-officinalis. Muret). Primevèro variable. Bois-noir, etc. Mars, avril.

P. farinosa L. P. farineuse. Vernayaz. Avril, mai.

P. viscosa Vill. P. visqueuse. Rochers du Trient. Avril, juin.

Androsace maxima L. Androsace majeure. Montorge, St-Lẻonard, Sierre, etc. Avril, mai.

Cyclamen Neapolitanum Ten. Cyclamen Napolitain. (G. hederæfolium Auct. non Ait.) Rochers de la Porte du Sceex, en face de la localité de Roche. Septembre, octobre.

\section{Amarautaceae. Amarantacées.}

Polycnemum arvense L. Polycnème des champs. Fouly, Vetroz. Juin, septembre. 


\section{Chenopodiaceae. Chenopodiacées.}

Blitum virgatum L. Blite effilée. Varonne. Juin, août. Chenopodium Botrys L. Anserine Botryde. Les Marques, Fouly, Sierre, Finges. Juillet, septembre.

C. opulifolium Schr. A. à feuilles d'Obier. Branson, Fouly. Juillet, septembre.

C. rubruum L. A. rouge. Bouveret, Branson, Charrat. Juillet, septembre.

C. ficifolium Sm. Bouveret. Juillet, septembre.

Polygoneae. Polygonées.

Rumex sanguineus L. Oseille sanguine. Vouvry, Martigny. Juin, août.

R. sculatus L. 0. scutifère. Rochers à St-Maurice. Juin, août.

Polygonum Lapathifolium L. Renouée à feuilles de $\mathrm{Pa}$ tience. Entre St-Léonard et Sion. Juillet, septembre.

\section{Laurineae. Laurinées.}

Laurus nobilis L. Laurier des poëtes. Vouvry, Sion. Sierre. Avril, mai.

\section{Santalaceae. Santalacées.}

Thesium intermedium Schr. Thésion intermédiaire.

Entre Charrat et Saxon. Mai, juillet.

\section{Eleagneae. Eléagnées.}

Hippophæ rhamnoïdes. L. Argoussier faux-nerprun. Bords du Rhone. Branson, etc. Avril, juin.

Euphorbiaceae. Euphorbiacées.

Euphorbia Gerardiana Jacq. Euphorbe de Gérard. Martigny, Branson, St-Léonard, Sierre. Juin. août.

\section{Moreae. Morées.}

Ficus Carica L. Figuier commun. Martigny, Saillon, Contey, Sion. Subspontané. Juillet. 


\section{Salicineae. Salicinées.}

Salix Daphnoides Vill. Saule Daphné. Çả et lả dans les haies du Bas-Valais. Mars, avril.

S. fragilis L. S. fragile. Bords du Rhône, entre Martigny et Branson. Mars, mai.

Populus alba L. Peuplier blanc. Charrat. Mars, avril. P. canescens Sm. P. grisâtre. St-Séverin. Mars, avril.

\section{Coniferae. Gonifères.}

Ephædra Helvetica Mey. Ephédra suisse (E. distachya Gaud. non L.) Folateires, Saillon, Montorge, Sion. Mai, juin.

Juniperus Sabina L. Genièvrier Sabine, Martigny, Fouly, Sierre. Avril, juin.

\section{Orchideae. Orchidées.}

Orchis militaris L. 0. militaire. Martigny. Mai, juin.

0. laxiflora Lam. 0. laxiflore. Sion. Mai, juin.

0. pallens L. 0. pâle. Gueuroz. Mai, juin.

0. sámbucina L. 0. sureau. Gueuroz, Branson. Avril, juin.

0. pyramidalis. L. 0. pyramidal. Branson. Juin, juillel.

0. odoratissima L. 0. odorant. Gueuroz, Fouly. Juin, juillet.

Herminium monorchis $\mathrm{R}$. Br. Herminie monorchide. Bords du Rhòne à Vouvry, Vernayaz. Mai, juillet.

Limodorum abortivum Sw. Limodore avorté. St-Maurice, Ardon (Haussknecht) et dans d'autres localités. Iuin.

Liparis Lœselii Rich. Liparis de Lœel. Près de Sion, élang de Montorge. Juin.

Spiranthes aestivalis Rich. Spiranthe d'été. Marais près de St-Naurice. Juillet, août. 
S. autumnalis Rich. S. d'automne. Au dessus de Branson. Juillet, septembre.

E. palustris Cr. E. des marais. Vouvry, Vernayaz, etc. Juin, juillet.

Cephalanthera rubra Rich. Céphalantère rauge (Epipactis rubra All.) Folateires. Juin, août.

\section{Irideae. Iridées.}

Iris Germanica L. Iris d'Allemagne. Sur les Rochers de St-Maurice, Martigny, Tourbillon et de Valère. Mai.

I. virescens DC. I. verdâtre (I. lutescens Gaud.) Rochers de Tourbillon. Avril, mai.

Gladiolus palustris Gaua. Glayeul des marais. Marais de Vouvry (Murith.) Juin.

Crocus vernus All. Safran printanier. St-Gingoiph, - St-Maurice. Mars, mai.

C. sativus All. S. cultivé. (C. aulumnalis Sut.) Je l'ai trouvé à Nontorge, à l'état sauvage, sur les rochers. On le cultivait à Sion et dans le Haut-Valais. Septembre, octobre.

\section{Amaryllideae. Amaryllidées.}

Lencoium vernum L. Niveole printanière, Perce-neige. Au-dessus de St-Gingolph, Monthey. Mars, arril. Narcissus biflorus Curt. Narcisse à deux fleurs. Près Sion, sous Valère. Avril, mai,

Galantus nivalis L. Galantine perce-neige. St-Maurice, Monthey. Mars, avril.

\section{Potamogetaceae. Potamogetacées.}

Polamogeton heterophyllus. Schr. Polamot heterophylle (P. ggraminus L.) Vernayaz, Martigny. Juin, août P. pusillus L. P. fluet, Vernayaz, le Guerset. Juin, août. 
P. marinus L. P. marin. Indiqué au Guerset par d'Angreville. Juillet.

\section{Lemnaceae. Lemnacées.}

Lemna gibba L. Lenticule gonflée. Vouvry. - été.

\section{Colchicaceae. Colchicacées.}

Bulbocodium vernum L. Bulbocode printanier. Mivellaz, Biranson, Fouly, Sion, St-Léonard, mayens de Sion. Février, mars.

\section{Asparageae. Asparagées.}

Ruscus aculeatus L. Fragon piquant, petit houx. Près de la cascade de Pissevache. Mars, avril Asparagus officinalis L. Asperge officinale. Martigny, Branson, Sion. Mai, juin.

Maianthemum bifolium D C. Muguet à deux feuilles. (Convallaria bifolia L.) Martigny, etc. Mai, juin.

\section{Liliaceae. Liliacées.}

Tulipa Oculus-Solis St-Anr. Tulipe œil-de-soleil. (T. maleolens Reich. T. Didieri Jord.) Entre Sion et le Rhône. Avril, Mai.

Scilla amœna L. Scille élégante. Ardon (d'Angreville). Avril, mai.

Gagea lutea Sch. Gagée jaune. (Ornithogalum sylvaticum Pers.) Au-dessus de St-Gingolph, Gueuroz. Avril.

G. arvensis Sch. G. des champs. Branson, Ardon. Mars.

G. saxatilis Koch. G. des rochers. (Ornithogalum bohemicum Gaud.) Branson, Valère. Mars.

Allium schænoprasum L. Ail Civelte, Branlettes. Con- tey. Juin, juillet.

A. acutangulum Reich, A. anguleux. St.-Haurice. Martigny, Sion. Juillel, août.

Endymion nutans Dum. Endymion penché (Hyacinthus 
non scriptus L.) indiquê par Murith à Montorge et à à Martigny. Mars.

Anthericum Liliago L. Anthéric faux-lis. Porte du Scex, Martigny, Branson. Mai, juin.

\section{Aroideae. Aroidées.}

Acorus Calamus L. Acore aromatique. Près de Saillou, Vouvry. Juin, juillet.

\section{Thyphaceae. Typhacées.}

Typha angustifolia L. Masselte à feuilles étroites. Riddes, Sion, Prazfalcon. Juin.

T. minima Hop. M. Naine, Sierre, Prazfalcon. Mai.

Sparganium minimum Fries. Rubannier nain. (S. natans auct. non L.) Vouvry (Reuter). Juin.

\section{Cyperaceae. Gypéracées.}

Cypertis longus L. Souchet long. Indiqué à Massonger. Août, septembre.

Cladium Mariscus R. Br. Cladie Marisque (Schœnus Mariscus L.). Sion, Finge. Juillet, août.

Scirpus maritimus L. Scirpe maritime. Etang de Ilontorge, Finge. Mai, août,

S. lacustris L. S. lacustre. Etang de Montorge, Guerset. Mai, juillet.

S. Iriqueter L. S. Iriquetre près de Fouly. Juin, août. S. acicularis L S. Epingle (Heleocharis acicularis R. $\mathrm{Br}$.). Guersel, Plan-Contey. Juin, août.

S. Duvalii Hopp. S. de Duval. Trouvé par M. Haussknecht au Bouveret. Juillet.

Carex nitida Host. Laiche lustrée. Branson, Sion, StLéonard. Avril, mai.

C. humilis Leys. L. naine. (G. clandestina Good.) Les Marques. Avril, mai.

C. gynobasis Vill. L. gynobase. Aven au-dessus de Sion, (Nurith): Avril, mai. 
C. pseudo-cyperus I. L. faux-souchet. Etang de Montorge (Reuter), Guerset (Murith). Juin.

C. riparia Gurt. L. des rives. Bords du Rhône, à Sion, etc. Nai, juin.

C. filiformis L. L. filiforme. Indiqué sur les bords du Rhône par Murith. Avril, mai.

\section{Gramineae. Graminées.}

Tragus racemosus Desf. Tragus en grappes. (Cenchrus

racemosus L.) Branson, Charrat, Fouly, Montorge, Sion, St-Léonard, Sierre.Juin, septembre.

Selaria verticillata $P$. B. var $\beta$ Rap. Sétaire verticillée.

(Panicum verlicillatum L. var.) Branson. Juin, juillet. Alopecurus geniculatus L. Vulpin genouillé. Vernayaz, Guerset (Murith). Mai, juillet.

Phleum asperum Vill. I hleole rude. Martigny, Sion. Mlai, juin.

P. Boehmeri Wib. P. de Boehmer (Phalaris phleoides L.) Branson, Montorge, St-Léonard. Juin, juillet. Apera interrupta P. B. Apère interrompue (Agrostis interrupta L.) Saxon, Charrat. Mai, juin.

Agrostis alba L. Schr. Agrostide blanche. Montorge, Vertroz. Juin, août.

Calamagrotis lanceolata Roth. Calamagrostis lancéolée (Arundo Calamagrostis L.) Vouvry. Juin.

Stipa capillata L. Slipe chevelue. Martigny, Sion, Sierre. Juin, août.

S. pennata L. S. plumeuse. Saint-Maurice, Martigny, Sion, etc. Mai, juin.

Kœeleria Valesiaca Gaud. Koelerie du Valais. Charrat, Montorge, Sion, St-Léonard, etc. Juin, août.

Aira præcox L. Canche hative, St-Léonard, Sion (Nurith). Nai, juin.

Avena falua L. Avoine folle. Martigny, Charrat, Contey. Juin, août. 
Trisetum Gaudinianum Boiss. Avoine de Gaudin. (Avena Lœfflingiana Gaud.) Près Outre-Rhône, Montorge, St-Léonard. Avril, mai.

Melica ciliata L. Mélique ciliée. Martigny, Sion. Mai, juin.

Sclerochloa dura P. B. Paturin dur (Poa dura Scop. Cynosurus durus L.) Charrat, Branson, Fouly, Montorge, St-Léonard. Avril mai.

Poa Eragrotis L. Paturin Eragrostide Eragrostis poaeides P. B.) Martigny, Charrat, Fouly, La Morge, Sion, Sierre. Juin, août.

P. pilosa L. Paturin poilu (Eragrostis pilosa P. B.) Sion. Juin, septembre.

P. concinna Gaud. P. mignon. Martigny, Branson, Saillon, Montorge, Sion, St-Léonard, Sierre. Avril, juin.

P. compressa L. P. comprimé. Fouly, Contey. Juin, juillet.

P. hybrida Gaud. P. hybride. Indiqué par M. d'Angreville à Gueuroz, dans le val d'lllier et dans le val d'Hèremence. Juin, juillet.

P. distans L. P. à fleurs écartées. Marais entre Martigny et Sion (Gaudin). Juillet, août.

Catabrosa aquatica P. B. Calabrose aquatique. (Aira aquatica L.). Entre Riddes et Econnaz. Mai, juin.

Molinia serotina M. et K. Molinie tardive. (Festuca serotina L.) Martigny, Sierre. Août, septembre.

Cynosurus echinatus L. Grételle hérissée. Branson, Riddes, Sion, Sierre. Juin, juillet.

Festuca rigida Kunth. Fétuque roide. (Poa rigida L.) Vouvry. Juin, juillet.

F. myuros Koch. Ehrh. Gaud. L. pro part. F. queue de rat. (F. pseudo-myuros S. W.) Monthey, Saxon. Mai, juin. 
F. sciuroides Roth. F. queue d'écureuil (F. bromoides L. Gaud.) Vétroz. Mai, juin.

F. Valesiaca Gaud. F. du Valais. (F. ovina et Valesiaca. Rap.) Les Marques, Branson, Charrat, St-Léonard, etc. Mai, juin.

F. glauca Lam. F. glauque. Martigny, Branson, etc. Mai, juin.

F. gigantea Vill. F. geante (Bromus giganteus L.) sous Montorge. Juin, juillet.

Bromus asper L. Brôme rude. Les Marques, Branson, elc. Juin, juillet.

B. inermis L. B, inerme. Branson (Gaudin) douteux. Juin.

B. tectorum L. B. des toits. Branson, Charrat, St-Léonard. Mai, juillet.

B. squarrosus L. B. squarreux. La Bâtia, Branson, Montorge, Sion, St-Léonard. Mai, juin.

B. arvensis L. B. des champs. Massongex, Contey. Juin, juillet.

B. secalinus L. B. Seigle. Champs à Branson, St-Léonard. Juin, juillel.

Agropyrum glaucum R. et S. Agropyre glauque (Triticum intermedium Host. Gaud.) Les Marques, Contey, Sierre. Juin, août.

Triticum nardus DC. Gaud. Froment faux-nard. (Micropyrum nardus Rap. Festuca tenuiflora Schr. Koch.) Montorge. Mai, juin.

Lolium rigidum Gaud. Ivraie roide. Montorge. Mai, juin.

\section{Characeae. Gharacées.}

Chara aspera willd. Charagne rude. Entre Sierre et Lœsche. Août.

C. hispida L. C. hispide. Martigny, Finges. Août. 


\section{Equisetaceae. Equisetacées.}

Equiselum ramosum Schl. Prêle rameuse. Saxon. Juillet, août.

\section{Filices. Fougères.}

Ophioglossum vulgatum L. Ophioglosse commun. Martigny, Fouly. Juin.

Ceterach officinarum C. B. Ceterach officinal. Rochers du Trient, Branson, Sion. Eté.

Polystichum Thelipteris Roth. Polyslic Théliptère. (Polypodium Thelipteris L.) Marais au-dessus de Vouvry. Été.

Asplenium Halleri DC. Doradille de Haller. Saint-Maurice, Martigny. Été.

A. Viride Huds. D. verte. Branson. Été.

A. Adianthum nigrum L. D. noire. Branson. Été.

A. septentrionale Sw. D. septentrionale. Pied du MontOttan, avant Martigny. Eté.

Scolopendrium officinarum Sw. Scolopendre officinal (Asplenium Scolopendrium L.) Porte du Scex, monthey. Été.

Adianthum Capillus Veneris L. Adianthe Capillaire. Ravoire de Marligny (Murith.) Été.

Lycopodiaceae. Lycopodiacées.

Lycopodiun inundalum L. Lycopode inondé. Marais du Val d'Illiers. Juillet, aoùt.

L. helveticum L. L. de Suisse. Pissevache. Gueuroz, Outre-Rhône, Branson. Juillet, août.

Marsileaceae. Marsileacées.

Marsilea quadrifolia L. Marsilée quadrifoliée. Marais à Vouvry. Juin, septembre.

Obs. Le Pilularia natans (?) est inciqué dans les fossés de Guerchet par M. d'Angreville. Juillet. 
On rencontre en outre, dans le Bas-Valais, les espèces suivantes.

Clematis vitalba L. Thalictrum aquilegifolium L. Anemone nemorosa L. Ranunculus Lingua L. R. Flammula L. R. acris L. R. nemorosus D G. R. repens L. R. bulbosus L. R. philonotis Ehr. R. arvensis L. Ficaria ranunculoides Roth. Caltha palustris L. Helleborus foetidus L. Aquilegia vulgaris L. Delphinium consolida L. Actaea spicata $\mathrm{L}$.

Berberis vulgaris L. Nymphaea alba L. Papaver Rhœas L. P. dubium L. Chelidonium majus L. Corydalis cava Wahl. Fumaria officinalis L.

Raphanus Raphanistrum L. Sinapis arvensis L. Erucastrum obtusangulum Reich. Diplotaxis muralis DG. D. tenuifolia DG. Barbarea vulgaris R. Br. Alliaria officinalis Andrz. Sisymbrium officinale Scop. S. Thalianum Gay. Nasturtium officinale R. Br. N. palustre DG. N. amphibium R. Br. Arabis hirsuta Scop. Cardamine pratensis L. G. amara L. C. hirsuta L. Alyssum calycinum L. Draba verna L. avec les formes de Jordan. Capsella Bursa-pastoris Mœnch. Thlaspi arvense L. T. campestre L. T. perfoliatum L. Iberis amara $\mathrm{L}$.

Helianthemum vulgare Gærtn. Viola hirta L. V. odorata L. V. canina L. V. tricolor L. et var. Reseda lutea L. R. Iuteola L. Parnassia palustris L. Monotropa hypopitys L. Polygala vulgaris L. P. comosa Schk. P. amara $\mathbf{L}$.

Dianthus prolifer. L. D. armeria L. D. sylvestris Jacq. Gypsophila saxifraga L. G. muralis L. Saponaria vaccaria L. S. officinalis L. S. ocymoides L. Silene inflata Sm. S. nutans L. Lychnis Flos-Cuculli L. L. Githago L. L. dioica L. Alsine tenuifolia Wahl. Arenaria scrpyllifolia L. Stellaria graminea L. S. media L. Malachium 
aquaticum Fries Cerastium glomeratum Thuill. C. triviale Link C. semidecandrum L. C. glutinosum Fries.

Linum tenuifolium L. L. catharticum L. Oxalis acetosella L. Malva rotundifolia L. M. sylvestris L. M. alcea L. Althaea hirsuta L. A. officinalis L. Erodium cicutarium l'Her. Geranium columbinum L. G. dissectum L. G. rotundifolium L. G. pyrenaicum L. G. pusillum L. G. molle L. G. Robertianum L. Tilia grandifolia Ehr. T. parvifolia Ehr. Hypericum tetrapterum Fries II. perforatum $L \mathrm{H}$. hirsutum $\mathrm{L}$. Vilis vinifera $\mathrm{L}$. var. sylvestris. Acer pseudoplaianus L. A. platanoides L. A. opulifolium Vill. A. campestre L.

Evonymus europacus L. Rhamnus catharticus L. R. Frangula L. Genista tinctoria L. Ononis spinosa I. O. repens L. Anthyllis vu!neraria L. Medicago falcata L. M. saliva L. M. lupulina L. M. minima L. Melilolus arvensis Wallr. (Trifolium Melilotus var. L.). M. officinalis Willd. M. albus Desr. Trifolium repens L. T. fragiferum L. T. scabrum L. T. arvense L. T. ochroleucum L. T. montanum L. T. medium L. T. pratense L. T. rubens L. T. alpestre L. T. aureum Pol. (agrarium Gaud.) T. agrarium L. (procumbens Gaud.). T'. procumbens L. (filiforme Gand.). Tetragonolobus siliquosus Schk. Astragalus glycyphyllos L. Coronilla Emerns L. C. varia L. Hippocrepis comosa L. Onobrychis sativa DC. Vicia tetrasperma Monch. V. hirsuta Koch V. Cracca L. V. sepium L. V. sativa L. V. angustifolia Roth. Lathyrus hirsulus L. L. pratensis L. L. sylvestris L. Orobus vernus L. 0. tuberosus L.

Gerasus avium D C. Prunus spinosa L. Spiræa Aruncus L. S. Ulmaria L Rubus cæsius L. R. fruticosus Gaud. Geum urbanum L. Fragaria vesca L. Potentilla Fragaria Sm. P. Anserina L. P. Tormentilla Nestl. P. replans L. P. verna L. P. argentea L. Agrimonia Eu- 
patoria L. Alchemilla arvensis Scop. Sanguisorba officinalis L. Poterium Sanguisorba L. Rosa canina L. R. arvensis Huds. R. rubiginosa L. R. sepium L. Cratægus oxyacantha L. C. monogyna Jacq. Pyrus communis L. P. malus L.

Epilobium hirsutum L. E. parviflorum Schr. E. palustre L. E. Letragonum L. Gircæa Luteliana L. Myriophyllum spicatum L. M. verticillatum L. Hippuris vulgaris L. Callitriche sessilis L. Ceratophyllum demersum L. C. submersum L. Lythrum Salicaria L. Myricaria germanica Desv. Portulaca oleracea L. Herniaria hirsuta L. Scleranthus annuus L.

Sedum acre L. S. reflexum L. S. album L. Ribes alpinum. L. Saxifraga aizoon L. S. Iridactylites L. S. rotundifolia L. S. aizoides L. Chrysosplenium aliernifolium L.

Hydrocotyle vulgaris L. Sanicula Europaea L. Heliosciadium nodiflorum Koch. Pimpinella saxifraga L. Egopodium podagraria L. Berula angustifolia Koch. Buplevrum falcalum L. Ethusa Cynapium L. Silaus pratensis Bes. Angelica sylvestris L. Heracleum sphondylium L. Pastinaca sativa $L \alpha$ opaca et $\beta$ pratensis. Dancus Carolta L. Torylis anthriscus Gm. T. helvetica Gm. Scandix pecten-Veneris L. Anthriscus sylvestris Hoffm. Chærophyllum temulum $\mathrm{L}$.

Hedera Helix L. Cornus sanguinea L. Adoxa moschatellina L. Viburnum Lantana L. V. Opulus L. Sambucus Ebulus L. S. nigra L. Lonicera periclymenum L. L. Xylosteum L.

Galium cruciata Scop. G. aparine L. G. uliginosum L. G. palustre L. G. boreale L. G. verum L. G. sylvalicum L. G. elatum Thuil G. erectum Huds. G. dumetorum Jord. G. sylvestre Pol. Asperula Cynanchica L. A. odorata L. Sherardia arvensis L. Valeriana ofticina- 
lis L. V. dioica L. Valerianella olitoria Pol. V. Morisonii DC. Dipsacus sylvestris Mill. D. pilosus L. Knautia arvensis Coult. K. sylvatica Dub. Scabiosa Columbaria $\mathrm{L}$.

Tussilago farfara L. Eupatorium cannabinum L. Senecio vulgaris L. S. viscosus L. S. erucæfolius L. S. Jacobæa L. S. aquaticus L. Jnula salicina L. J. Conyza DC. J. dysenterica L. Solidago virga aurea L. Bellidiastrum Michelii Cass. Erigeron Canadensis L. E. acris L. Gnaphalium uliginosum L. Filago Germanica L. var canescens et var lutescens Jord. F. arvensis L. F. montana L. F. Gallica L. Artemisia vulgaris. L. A. campestris L. Matricaria Chamomilla L. M. Parthenium. L. Ghrysanthemum inodorum L. C. leucanthemum L. Bellis perennis L. Anthemis arvensis L. Achillea ptarmica L. A. millefolium L. Bidens cernua L. B. tripartita L. Onopordum acanthium L. Carduus nutans L. C. defloratus L. Girsium lanceolatum Scop. C. palustre Scop. G. acaule All. G. oleraceum Scop. G. arvense Scop. Carlina vulgaris L. Lappa minor DG. Serratula tinctoria L. Centaurea jacea L. C. amara L. G. Scabiosa L. C. Cyanus L. Lampsana communis L. Cicorium Jntybus L. Taraxacum Dens Leonis Desp. T. laevigatum DG. T. palustre DG. Prenanthes muralis L. P. purpurea L. Sonchus arvensis L. S. oleraceus L. S. asper Vill. Crepis fœtida L. C. taraxacifolia Thuil. G. biennis L. C. virens Vill. Hieracium Pilosella L. H. Auricula L. H. praealtum Vill. H. florentinum All. H. staticæfolium All. H. murorum L. et var. H. sylvaticum Lam. H. sabaudum L. H. umbellatum L. Hypochaeris radicata L. Tragopogon pratensis L. Picris hyeracioides L. Trincia hirta Roth. Apargia autumnalis L.

Jasione montana L. Phyleuma spicatum L. Prismatocarpus speculum L'Her. Campanula rotundifolia L. C. 
patula L. G. rapunculus L. C. glomerata L. Calluna vulgaris Sal. Ilex aquifolium $L$. Ligustrum vulgare $\mathrm{L}$. Fraxinus excelsior L. Gynanchum Vince toxicum R. Br. Vinca minor L. Gentiana cruciata L. G. ciliata L. Erythraea Centaurium Pers. Convolvulus sepium L. C. arvensis L. Cuscuta Europaea L. G. Epithymum L. C. Trifolii Bab. Lithospermum arvense L. L. officinale L. Pulmonaria officinalis L. P. angustifolia L. Myosotis palustris With. M. coespitosa Sch. M. intermedia Link. Symphytum officinale L. Anchusa Italica Retz. Lycopsis arvensis L. Borago officinalis L. Cynoglossum officinale L. Solanum dulcamara L. S. nigrum L. Hyoscyamus niger L. Verbascum Thapsus L. V. Lychnitis L. V. nigrum L. V. Blattaria L. Scrophularia nodosa L. S. aquatica L. Antirrhinum Orontium L. Linaria spuria Mill. L. Elatine Mill. L. minor Desp. L. vulgaris Mill. Melampyrum cristatum L. M. arvense L. M. pratense L. Rhinanthus Crista-Galli L. R. minor Ehr. Euphrasia odontites L. et var. verna. E. officinalis L. et var. Veronica Anagallis L. V. Beccabunga L. V. chamaedrys L. V. officinalis L. V. spicata L. V. serpyllifolia L. V. arvensîs L. V. agrestis L. V. hederaefolia L. Orobanche minor Sut. 0. Galii Vauch.

Mentha sylvestris L. M. aquatica L. M. arvensis L. M. Pulegium L. Lycopus Europaeus L. Thymus serpyllum L. Calamintha Acinos Clairv. G. officinalis Mœnch. Clinopodium vulgare L. Salvia pratensis L. Glechoma hederacea L. Galeopsis angustifolia Ehr. G. Tetrahit L. Lamium amplexicaule L. purpureum L. L. maculatum L. Galeobdolon luteum Huds. Leonurus Cardiaca L. Stachys sylvatica L. S. palustris L. S. recta L. S. annua L. Betonica officinalis L. Ballota foetida Lam. Melittis melissophyllum L. Prunella vulgaris L. P. laciniata L. P. grandiflora Jacq. Scutellaria galericulata L. 
Ajuga reptans L. A. Genevensis L. A. chamaepitys Sch. Teucrium Scorodonia L. T. Botrys L. T. Scordium L. T. charnaedrys L. T. montanum L.

Verbena officinalis L. Pinguicula vulgaris L. Primula acaulis L. P. elatior Jacq. P. officinalis Jacq. Lysimachia vulgaris L. L. nemorum L. L. nummalaria L. Anagallis phœnicea Lam. A. cœrulea Schr. Globularia vulgaris L. Plantago major L. P. lanceolata L.

Amaranthus Blitum L. Chenopodium polyspermum L. G. fœtidum Lam. C. album L. G. hybridum L. G. urbicum L. C. murale L. C. glaucum L. C. Bonus-Henricus L. Atriplex patula L. Rumex conglomeratus Muŕ. R. pulcher L. R. obtusifolius L. R. crispus L. R. acetosa I. R. acetosella L. Polygonum amphibium L. P. lapathifolium L. P. persicaria L. P. mite Schr. P. hydropiper L. P. aviculare L. P. convolvulus L. P. dumetorum L. Stellera Passerina L. Daphne mezereum L. D. Laureola L. Thesium pratense Ehr. Aristolochia Clematitis L. Euphorbia Helioscopia L. E. platyphylla L. E. stricta L. E. dulcis L. E. palustris L. E. sylvatica Jacq. E. Cyparissias L. E. Peplus L. E. falcata L. E. exigua L. Mercurialis perennis L. M. annua L. Buxus sempervirens L. Urtica dioica L. U. urens L. Parietaria officinalis L. Iumulus Lupulus L. Ulmus campestris L. Norus alba L. Juglans regia L.

Fagus sylvatica L. Castanea vulgaris Lam. Quercus pedunculata Ehr. Q. sessiliflora Sm. Q. pubescens Willd. Corylus Avellana L. Carpinus Betulus L. Salix purpurea L. S. viminalis L. S. nigricans Fries. S. Capraea L. S. cinerea L. S. repens L. S."alba L. S. vitellina L. S. Babylonica L. S. triandra L. Populus tremula L. P. nigra L. P. pyramidalis Ros. Betula alba L. Alnus incana DC. A. glutinosa Gærnn. Taxus baccata L. Juniperus communis T. Pinus sylvestris L. P. abies L. P. Picea L. P. Larix L. 
Tamus conmunis L. Orchis morio L. 0. coriophora L. 0. ustulata L. O. mascula L. 0. latifolia L. 0. maculata L. Aceras anthropophora R. Br. Ophrys aranifera Huds. 0. arachniles Hoffm. 0. apifera Huds. 0. myodes Jacq. Neottia Nidus-avis Rich. Listera ovata R. Br. Epipactis latifolia All. E. atrorubens Hoffm. Cephalanthera grandiflora Bab. C. ensifolia Rich. C. rubra L. Iris Pseudo-Acorus L.

Alisma Plantago L. Triglochin palustre L. Potamogeton densus L. P. natans L. P. fluitans Roth. P. lucens L. P. perfolialus. L. P. crispus. L. P. pectinatus I. Zanichella palustris L. Lemna minor L. L. trisulca L. L. polyrhizza L.

Colchicum autumnale L. Tofieldia palustris Huds. Convallaria verlicillata L. C. polygonatum L. C. multiflora L. C. maialis L. Paris quadrifolia L. Scilla bifolia L. Ornithogalum pyrenaicum L. 0. umbellatum L. Allium sphaerocephalum L. A. vineale L. A. olcraceum L. A. carinatum L. A. ursinum L. Muscari comosum Nill. M. racemosum Mill. Anthericum ramosum L.

Juncus conglomeratus L. J. effusus L. J. glaucus Ehr. J. compressus Jacq. J. bufonius L. J. obtusiflorus Ehr. J. lamprosarpos Ehr. Luzula vernalis DC. L. campestris DG. Arum maculatum L. Typha latifolia L. Sparganium ramosum Huds. S. simplex Huds.

Cyperus flavescens L. C. fuscus L. Schænus ferrugineus L. Scirpus compressus Pers. S. sylvaticus L. S. supinus L. S. panciflorus Light. Heleocharis palustris R. Br. Eriophorum latifolium Hopp. Carex Davalliana Sm. G. disticha Huds. (intermedia Good.). C. vulpina L. C. muricala L. C. teretiuscula Good. C. paniculata L. C. leporina L. C. remota L. C. stricta Good. C. alba Scop. G. panicea L. C. glauca Scop. C. maxima Scop. C. pallescens L. C. ornithopoda Wild. 
G. tomentosi L. C. montana L. G. flava L. C. sylvatica Huds. G. Ederi Ehr. C. fulva Good. C. distans L. C. ampullacea Good. C. vesicaria L. C. paludosa Good. C. riparia Curt. C. filiformis L. G. hirta L.

Andropogon Ischaemum L. Panicum sanguinale L. P. Crus-Galli L. Setaria glauca P. B. S. viridis P. B. Phalaris arundinacea L. Anthoxanthum odoratum L. Alopecurus agrestis L. A. fulvus Sm. Phleum pratense L. et var. Cynodon dactylum Pers. Agrostis canina L. Apera spica venti P. B. Calamagrostris littorea DG. Phragmites communis Trin. Koeleria cristata Pers. Avena pubescens L. A. pratensis L. A . flavescens L. Holcus lanatus L. H. mollis L. Arrhenaterum elatius M. et K. et var. Melica nutans L. Dactylis glomerata L. Poa annua L. $P$. bulbosa $L$. et v. vivipara $P$. nemoralis $L$. et var. P. pratensis L. P. trivialis L. Glyceria fluitans R. B. Molinia cærulea Kæl. Briza media L. Cynosurus cristatus L. Festuca ovina L. F. duriuscula L. F. heterophylla Lam. F. arundinacea Schr. Bromus erectus Huds. B. sterilis L. B. mollis. L. B. racemosus L. B. secalinus. L. Secale cereale L. Agropyrum repens. P. B. A. caninum $R$ et $S$. Brachypodium sylvaticum $R$. et S. B. pinnatum P. B. Lolium perenne L. L. mulliflorum Gaud. L. temulentum L. Hordeum murinum L.

Chara fœetida A. Br. Equisetum arvense L. E. Telmateia Ehr. E. palustre L. E. hyemale L. E. limosum. L. Asplenium trichomanes L. A. Ruta-muraria L. Pteris aquilina $\mathbf{L}$. 


\section{NOTES}

\section{sur les espèces rares ou critiques}

\section{et sur les plantes qui, pour la Suisse, ne se trouvent que dans le Bas-Valais.}

Les plantes spéciales sont désignées par l'astérisque •

Clematis recta L. (C. erecta All. DC. Prodr.) Celte espéce méridionale ne se trouve en Suisse que dans le canton du Valais à Saillon, Vetroz, Avent et dans celui du Tessin. En Allemagne elle existe de Wurzbourg à Francfort, en Autriche, en Bohême, etc. Elle habite surtout le Piémont, le midi de la France etles PyrénéesOrientales.

* Thalictrum minus L. $\gamma$ glandulosum Koch. (Th. pubescens Schl. T. foetidum Gouan non L.) Taillis près des Folateires. M. Boissier (voyage en Espagne) dit que cette variété habite la France méridionale, l'Espagne, l'Italie méridionale et le Valais? Cependant je crois bien que c'est la forme que nous possédons à Branson, dans la vallée de Saas et de Zermatt. D'après M. Boissier sa seule pubescence glanduleuse la distingue du T. minus $I_{\lrcorner}$, tandis que ses étamines non pendantes, ses folioles plus grandes et plus allongées, à nervures très-proéminentes en dessous, empêchent de la confondre avec le T. totidum. Notre plante n'est pas indiquée en Suisse ailleurs qu'en Valais.

T. fœtidum L. Cette espèce qui croît à Branson, à 
Sion et surtout dans les vallées de Saas et de Zermatt, se retrouve dans le Canton de Berne et celui des Grisons; elle habitr, en outre le Tyrol, la Bohème, le Piémont et le Dauphiné.

Anemone Pulsatilla L. var, nutans Gaud. (A. monIana Hopp. Koch. Rap. Greml., etc.) Cette forme remarquable couvre presque toutes les collines du Bas-Valais, Martigny, Branson, Charrat, Sion, Sierre, elc.; on ne la retrouve en Suisse que dans les Grisons. Hoppe a décrit el figuré sous le nom d'Apemone montana (ap. Sturm Deutschl. Flora, Div. 1 Tab. XIII) une plante qui croit dans les bois de Lipizza, près de Trieste; il dit positivement que sa plante a la fleur dressée-étalée, que lıs pétales sont droits, lancéolés, acuminés, caractères qui ne se rapportent en aucune façon à notre plante du Valais dont les fleurs sont de médiocre grandeur, penchées, d'un violet foncé et noirâtre et dont les pétales sont courts et ohtus. Celte forme habite le Tyrol et le midi de la France. L’A. Pulsatilla L. var. præcox Gaud est beaucoup plus répandue; en Suisse elle croît dans las cantons de Vaud, de Zurich et d'Argovie; elle habite presque toute l'Europe, Sibérie, Allimagne, Angleterre, France, etc.

Voici du reste les caractères qui différencient ces deux formes. La Precox a les lanières des feuilles linéaires étroites, la Nutans les a sensiblement plus lar'ges el plus longues : la Priecox a ses sépales elliptiques oblongs ou lancéølés, la fleur dressée, grande, violette et de couleur lilas quand on la regarde par transparence; la Nutans a les sépales oblus, la fleur penchéc, plus petite, d'un violet noirâtre el rouge quand on la regarde par transparence. La première fleurit plus tôt que la seconde.

* Adonis autumnalis L. Plante erratique qu'on trouve 
çà et là dans les cultures du Bas-Valais oủ elle est plus rare que l'A. æstivalis L. et l'A. flammafa Jacq. On la rencontre dans les moissons de presque toute l'Europe ; elle paraît originaire de la Grèce où elle croît à Zante dans les prés (DC. Gẻogr. Bot.) Elle n’a pas été indiquée ailleurs en Suisse.

Adonis æstivalis L. (A. ambigua Gaud.) Branson, Sion, St-Léonard, etc. Plante cultivẻe involontairement dans les champs de blé du Bas-Valais, du canton de Bâle et de l'Engadine; on la retrouve en Savoie, en Piémont, en France el en Italie. Elle se reconnaît à sa corolle grande et ordinaïrement d'un rouge vif et à ses fruits formant un épi compact, à son style entièrement vert.

Adonis flammæa Jacq. (A. æstivalis Gaud.) Branson, Montorge, St-Léonard, etc. Mêmes obsarvations que pour la précédente dont elle se distingue par ses fleurs moins grandes, d'un rouge orangé, dont les pétales sont souvent avortés, par ses fruits formant un épi lâche, plus allongé, et par son style noir au sommet.

* Adonis vernalis $\mathrm{L}$. Cette belle espèce est spéciale au Valais. Les Folaleires, Charrat, etc. Elle habite la Sibérie, la Taurie, l'Allemagne, la France et l'ltalie. Elle réussit très-bien dans les jardins.

* Ranunculus gramineus I. n'a été trouvé que sur la colline de St-Léonard, localité unique pour la Suisse. Il habile le Piémont, la Sardaigne, I'Espagne, le Portugal, le midi et le centre de la France, ainsi que le pays de Galles en Angleterre; il manque en Allemagne et dans le nord de l'Europe. C'est une des rares plantes de notre pays.

Glaucium luteum Scop. (G. flavum Crantz. Chelidomum Glaucium L.) Cette espèce, indiquée par Murith 
et Gaudin, au bord de l'étang de Montorge, se retrouve abondamment dans le gravier des rives du lac de Neuchâtel à Corcelettes et à la Poissine. Elle habite le bord des mers, des lacs, des étangs et des rivières de l'Europe centrale et méridionale, (Allemagne, France, Espagne), se r'etrouve en Angleterre, en Danemarck, en Asie Mineure, dans l'Afrique septentrionale, aux Canaries et dans l'Amérique du Nord. (Boissier.)

* G. corniculatum Curt. (G. violaceum Juss.) Celte rare espèce n'a été trouvée qu'en petite quantitẻ dans les champs de Valère à Sion, et ceux de Montorge et de St-Léonard. Elle habite les moissons de l'Autriche, de la Bohême, du midi de la France et de deux localités d'Angleterre. On la retrouve en Espagne, aux Canaries, dans l'Afrique septentrionale, en Asie Mineure et en Syrie. (Boissier):

* Corydalis solida Sm. $\beta$ australis Haussm. ex Reut. (G. solida Sm. var. floribus albis Rap). Cette intéressante variété a été découverte par M. Reuter, à Gueuroz et à Branson; elle n'a pas été signalée dans d'autres localités suisses. Volci la note que je dois à mon ami Reuter: a La Corydalis solida que j'ai recueillie, à Branson et à Gueuroz, m'a paru diffẻrer de celle qui croìt à Genève et en deçà des Alpes par sa grappe de fleurs plus lâche, d'une couleur blanche avec les lèvres roses ou entièrement d'un rose pále, les siliques sont plus longues et plus étroites. C'est tout à fait la plante qui croît dans le Tyrol méridional, prés de Botzen, et que $M$. Haussmann décrit comme var. $\beta$ australis (Fl. Tyrol p. 41). D'après lui, le limbe du pétale supérieur est plane et non roulé en corne; mais ce caractère ne me paraît bien marqué ni dans la plante du Tyrol, ni dans celle du Valais. La silique est plus longue et plus étroite, les graines sont 
unisériées. J'aì vu la même plante de Dalmatie, et dans les Contributions à la Flore de Menton, un beau dessin, sous le nom de Corydalis solida, qui représente parfaitement l'espèce valaisanne. La Corydalis solida de Genève a constamment les fleurs d'un rouge vineux, ne variant jamais au blanc ni au rose pâle; elles forment une grappe compacte, inclinée et unilatérale, les lobes des lèvres sont fortement révolutés, les siliques sont plus courtes et plus larges que dans la var. Australis, et, le plus souvent, à graine bisériées. Notre plante de Genève est bien l'espèce décrite par D G., sous le nom de C. bulbosa, et par Gaudin et Koch, sous celui de G. solida; elle croît dans tout le centre et le nord de l'Europe, et s'élève sur les Alpes et le Jura. Je l'ai observée le printemps dernier, en immense quantité dans le vallon d'Ardran, près du Reculet, avec Scilla bifolia, Narcissus Pseudo-Narcissus, Gagea lutea, Crocus vernus, etc., etc. ” (Reuter).

Erysimum Helveticum DC. Gaud. (Cheiranthus Helveticus Jacq. G. pallens. Hall. fil. E. Cheiranthus Pers. var. a Moritzi). Abondant sur les collines du BasValais, Branson, Contey, Montorge, Sion, St-Léonard, Sierre, Varonne, elc., On le retrouve dans le Tessin et dans les Grisons. Il manque en France, en Allemagne, en Angleterre, et n'est indiqué d'une manière sûre qu'en Piémont et dans les Carpathes. C'est donc une des plantes rares de la Suisse.

E. virgatum Roth. Cette plante, qui croît près de Charrat et de Saxon, se retrouve dans le Jura Neuchâtelois et dans les Grisons. Elle habite la France et l'Allemagne.

* Sisymbrium Pannonicum Jacq. Gaud. (S. Sinapistrum Crantz). Cette espèce rare et spéciale au Valais, 
n'a été signalée qu'à Iserabloz au-dessus de Riddes, dans le val d'Anniviers et à Stalden. Elle habite surtout l'Allemagne, la Prusse, Francfort-sur-l'Oder, la vallée du Rhin de Strasbourg à Manheim, la Bohême, l'Autriche, la Hongrie et la Ligurie. Pour la France elle n'est indiquée que sur les rochers de grès Vosgien à Mutzig. Elle manque en Angleterre, en Espagne, en Italie.

* Clypeola Jonthlaspi L. (C. Gaudini Trachs.) Cetle petite plante spéciale au Bas-Valais, oủ elle est rare, n'a été trouvée qu'à Saillon, à Sion. (Tourbillon et Valère), et près de l'Ermitage de Longeborgne au-dessus de Bramois. En Allemagne elle n'est indiquée que dans la basse Autriche et à Fiume; en France, elle habite le Dauphiné, le Languedoc, la Provence, la Corse, etc., elle se retrouve en Espagne, dans l'Afrique septentrionale et en Perse.

* Vesicaria utriculata Lam. (Alyssum utriculatum L.). Celte espèce peut être considérée comme spéciale au Bas-Valais, bien qu'on l'ait trouvée près de Lavey, sur la frontière du canton de Vaud. Je l'ai prise sur les rochers, à côté de la cascade de Pissevache; il y en a de superbes touffes près du Trient, en montant à Gueuroz, mais il est rare qu'on puisse les atteindre. Elle habite la Dalmatie, le Piémont, la Savoie, la France méridionale, l'Italie et la Grèce. Elle manque en Angleterre et dans tout le nord de l'Europe.

Camelina sylvestris Wallr. (G. microcarpa Andrz.) Encore une espèce erratique, occupant avec ses congénères les cultures et les champs. On la trouve çà et là, à Martigny, Branson, Charrat, Montorge, Saint-Léonard, etc.. a Ollon au canton de Vaud, et probable- 
ment ailleurs. Elle habite les cultures de presque toute l'Europe. Du reste, les Camelina sont originaires de la Russie méridionale et du Caucase.

Calepina Corvini Desv. (Bunias Cochlearioides Mur.) Espèce erratique rencontrée à St-Maurice, et reparaissant depuis un très-grand nombre d'années dans les cultures du village de Branson. Elle a été trouvée autrefois à Genève, mais elle a disparu; elle est indiquée dans les cantons de Vaud et de Neuchâtel. Elle habite les champs du midi et du centre de la France, de la Hongrie, de la Ligurie, du Piémont, de l'Espagrne, etc.; elle est spontanée dans les lieux humides des déserts au nord de la mer Caspienne, dans les prés à Zante, en Sicile et en Italie; c'est dans les temps modernes qu'elle s'est répandue dans les champs de l'Europe, oủ elle est tantôt naturalisée tantôt adventive. (A. D G. Geog. Bot.).

* Helianthemum salicifolium Pers. ne se trouve que sur les pelouses, près de Branson, seule localité connue en Suisse. Hegestschweiler l'avait indiqué au Tessin, mais M. Muret l'a cherché inutilement. Il habite l'Istrie, la Dalmatie, l'Italie, la Grèce, la Corse, le midi de la France, l'Asie Mineure et le nord de l'Afrique. Le Valais serait donc la station la plus septentrionale de cette espèce.

Viola Sciaphila Koch. Cette espèce a été trouvée par M. Rapin, en montant à Joux-Brulée, au-dessus de Branson. On la reconnaît à ses ovaires très-glabres, à ses capsules ovales oblongues, à ses feuilles ovales largement cordiformes, presque glabres. Les fleurs sont violelles, avec la gorge blanche, faiblement odorantes. La plante est dépourvue de stolons. Koch l'indique en Suisse et dans le Tyrol. M. Rapin l'a récoltée 
dans le Jura (Grêt du Miroir), au Plan de Jaman, etc. D'après lui, c'est la même planie que la V. pyrenaica Ram. in D C. fl. fr. et Prodr et in Herbar. Ce serait aussi la même que la V. glabrescens Falk. On l'a trouvée dans les cantons de Neuchâtel et des Grisons.

Obs. L. V. Stevenii Bess. trouvée à Montorge par M. Haussknecht, et à Sion par M. Rapin, est une plante qui paraît différer de la V. odorata, et qu'il faudrait étudier.

Viola arenaria DC. se trouve à Mivellaz, aux Folateires, à Saxon, etc. Gaudin n'indique pas d'autres localités suisses, cependant on l'a signalée dès lors dans les cantons de Berne, d'Uri, de Zurich et des Grisons. Elle habite l'Allemagne, la Bohême, les Alpes du Dauphiné, la Savoie, le Piémont, le Caucase, etc.

* Lychnis Coronaria Lam. Agrostemma Coronaria. L. Cette belle fleur, souvent cultivée dans les jardins, ne se trouve qu'au-dessus de Branson et de Fouly. Elle habite l'Allemagne méridionale, la Hongrie, la Dalmatie, le Piémont, et quelques localités de la France.

* Buffonia macrosperma Gay. Gr. et Godr. (B. tenuifolia Vill. Gaud .Reich. ic. non L. B annua D G.). Voici la description de cette espèce d'après la Flore de France de Grenier et Godron. Plante annuelle irrégulièrement rameuse, étalée dès la base; feuilles subuléessétacées. Inflorescence en thyrse formé de petites cimes de 2 à 3 fleurs dont la centrale est plus longuement pédicellée; pédicelles scabres. Sépales lancéolés-acuminés, à 5 nervures prolongées presque jusqu'au sommet. Pétales oblongs, d'un tiers plus courts que le calice. Quatre étamines à filets atteignant à peine le quart des sépales. Styles trẻs-courts, à peines égaux aux filets. Graines grosses, doubles de celles de la Buffonia tenuifolia L., obovées-allongées fortement tu- 


\section{7}

berculeuses sur le dos et sur les faces. Cette description convient en tous points à notre plante dı Valais qui ne croît pas ailleurs en Suisse et qu'on rencontre à Charrat, dans les graviers ide la Morge, près d'Ardon, à Sion et surtout à Sierre et à Varonne. Ell ‘ habite le centre et le midi de la France, le Piémont et l'Espagne; elle est indiquée avec doute en Angleterre, et parait manquer dans le nord de l'Europe. Le genre a été créé par Sauvages et adopté par Linné qui en a accepté la paternité. Dédié au célèbre Buffon en ces termes: - Dedicato illustrissimo Horti Regii Parisiensis Præfecto et Acad. Regiæ scient. Paris. Sodali D. de Buffon; " il est cependant écrit, par erreur, dans le texte Bufonia, avec une seule f. (Note de M. le Dr Müller, conservateur de l'Ilerbier De Candolle). La même erreur a dû se zommettre pour le Genre Valantia, créé par Tournefort, adopté pas Linné, et qui devrait s'écrire Vaillartia du nom de Sébast. Vaillant à qui il fut probablement dédié.

Tout cela scrait de mince importance si quelques personnes n'avaient pas eu la malencontreuse idée de prêter gratuitement à Linné l'intention de faire un pitoyable jeu de mols en attribuant au nouveau genre, comme étymologie, le mot Bufo qui signifie Crapaud et cela par jalousie à l'endroit du célèbre naturaliste français.

Or, qu'a-t-il dû se passer réellement? Voici ce qui me parait ressortir tout simplement des faits. Sauvages le véritable père du Genre, écrit dans son texte Bufonia - faute typographique manifeste - puisqu'il adresse sa dédicace à Buffon et qu'il imprime dans l'Index : Buffonia. Linné, le père adoptif, donne le mot comme il l'a reçu, et il est imité par tous les auteurs contemporains qui respectaient jusqu'aux erreurs des 
maîtres (sauf Gronovius dans son Auctuarium in Bibliot. Botanicam). Ainsi Gouan et Lamarck en France écrivent Bufonia, le Bufon, la Bufone; Allioni et Murith font de même, etc. Enfin de Candolle dans la Flore française rétablit la véritable orthographe: Buffonia, et dès lors les auteurs modernes en ont fait autant. J'en excepte sir William Hooker qui, dans sa British Flora, persiste à écrire Bufonia qu'il traduit par Toad-grass (Herbe au crapaud). Il ajoute en note ${ }^{1}$ que le nom de de la plante vient, suivant quelques-uns, de ce qu'elle croît dans des places fréquentées par les crapauds. (Ce qui est parfaitement inexact.) Smith, de son côté, dit quele nom a été donné par Sauvages en l'honneur de Buffon qui n'avait en vérité que de très-minces prétentions à un honneur botanique, circonstance qu'on suppose avoir élé indiquée par Linné au moyen dunom spécifique tenuifolia. Voilì bien du verbiage à propos d'une malheureuse lettre. (Nuch ado about nothing) Peut-être, mais j'en ai le cœur not et je termine en répétant avec Richter ${ }^{2}$ : Que ceux qui prétendent que Linné a changé le nom en Bufonia par méchanceté, prouvent leur assertion ${ }^{3}$.

Ruta graveolens L. La Rue croît dans le Bas-Valais sur les rochers de St-Maurice en montant à l'Ermi-

${ }^{1}$ Bufonia, indeed, is the original spelling, and so called, some say, from the plant growing in places frequented by toads

Name given by Sauvages in honour of the celebrated Buffon " who had indeed very slender pretensions to botanical honour; a circumstance supposed to have been indicated by Linnaeus in the specific name tenuifolia. $(\mathrm{Sm}$.

${ }^{2}$ Nomen, iniqua mente, a Linnaeo in Bufoniam mutatum esse, probent ii, qui narrant. (Richter).

3 J'ai lu jadis une dissertation sur le même sujet, mais je ne me rappelle ni le nom de l'ouvrage, ni celui de l'auteur que je ne puis citer. 
tage et aux environs de Sierre ; on la retrouve à Neuchâtel et dans le Tessin. Elle habite le midi de la France, le Piémont, quelques localités de l'Allemagne et le Tyrol méridional. Je la crois spontanée dans le Bas-Valais.

Rhus Cotinus L. Le Fustel ne croit à l'élat sauvage qu'à Martigny et à Louesche la Ville, en Valais; on le retrouve dans le canton du Tessin. II habite les collines sèches du midi de la France, le Piémont où il est abondant, l'Autriche, le Tyrol et le Littoral de l'Adriatique. C'est une des plantes méridionales du BasValais qu'on cultive sourent dans les bosquets.

* Genista radiata Scop.. (Cytisus radialus lioch, Spartium radiatum L.) Celle espèce rare se trouve près d'Ardon, dans le ravin de la Sionne, ả Lens et à Saviège; on ne l'a pas rencontrée ailleurs en Suisse. Elle habite la Ilongrie, la Carniole, le Frioul, l'Italie, et pour la France, le Dauphiné et les Basses-Alpes. Elle parait manquer dans le reste de l'Europe.

Genista ovata Mur. Gaud. non W et K. Voici ce que je trouve dans le supplément de la Flore Suisse de A. Gremli. La localité du Valais n'a pas été retrouvée, (ce serail à la Crotta, entre St-Maurice et Outre-Rhòne.) En revanche, elle croit dans le Tessin et le canton de Schaffouse. Ce ne serait pas la plante de IIongrie, mais bien le G. Peireymondi Lois. (G. lincloria var. lasiocarpa Gr. et Godr.) D’après le Prodrome, l'espèce décrite par Waldstein et Kitaibel, habite la Slavonie, le Banat, le Piémont et l'Italie., La variété lasiocarpa de Gr. et Godr. n'est indiquée par eux que dans la vallée de Thorenc, près de Grasse. Elle se distingue du G. tincloria type par sa lige couverte de poils étalés et par ses fruits velus-tomenteux. 
Ononis altissima Lam: (0. hircina Jacq. 0 . fœlens All.) Celte belle espèce croît dans les prairies de Charrat et de Saxon, et probablement dans d'autres localités. Pour la Suisse, elle est indiquée en outre dans le canton du Tessin. Elle habite l'Allemagne et le Piémont, et parait manquer en Angleterre et en France du moins elle n'est pas mentionnée dans Flore de Grenier el Godron.

* Trigonella Monspeliaca L. Abondante à Tourbillon, plus rare sur les côteaux de Branson, de Foully el de Saillon, celte petite plante, qui est ordinairement mêlée avec la Medicago minima, est spéciale au BasValais. Elle habite la Hongrie, l'Autriche, l'Istrie, le midi et le centre de la France, ainsi que l'Espagne, le Portugal, l'Italie, la Grèce, la Georgie, et la Barbarie (Boissier).

Oxytropis Halleri Bung. (0. Uralensis I) G. Gaud., Astragalus Uralensis Jacq. La plante de l'Oural diffère de l'espèce européenne). Pour le Valais, elle croît aux Folateires, á Charrat, à Saxon, à Sierre; on la retrouve en Suisse à la dent de Jaman, sur les Alpes de Gruyère (Rapin), à Lucerne et dans les Grisons, du reste elle habite le Tyrol, la Carinthie, la Hongrie, le Piémont, l'Italie supérieure, les Pyréné ss Orientales et Centrales, les Alpes du Dauphiné et les montagnes de l'Eccsse.

* Coronilla coronata D C. Gaud. non L. (C. minima L. non DG. C. minima L. $\beta$ lotoides Koch, C. minima L. $\beta$ australis Gr. Godr. C. montana Murith non Scop.) Il paraîtrait, d’après les localités el les figures citées par Linné, que sa C. coronata serait la G. vaginalis Lam, et C. minima D C., tandis que sa C. minima serait bien la C. coronata DG. Cette espèce, critique et 
rare, ne croît qu'aux environs de Varonne, au-dessus de Sicrre. D'après M. Boissier (voyage en Espagne), elle habile les montagnes de l'Europe, l'Espagne, le midi de la France, le royaume de Naples et le Valais.

* Onobrychis arenaria D C. (U. supina Gaud.) Croît à Branson, Saxon, Montorge, St-Léonard et Sierre; n'est indiqué par les auteurs allemands et par le Prodrome, qu'à Trieste, en Hongrie, el en Sibérie; parait manquer en France, en Angleterre, et dans le reste de l'Europe.

* Vicia pisiformis L. Croît dans un bois au-dessus du village de Fouly, seule localité certaine pour la Suisse, bien qu'elle ait été indiquée à Ferreste (Pfirt) près de Bâle. Elle habite les bois montagneux de l'Allemagne et du nord-Est de la France; n'est pas mentionnée en Angleterre.

Vicia Gerardi DG. (V. Cracca L. var. Gerardi Gaud.) Croît dans les haies et les buissons à Charrat, Saxon et dans la vallée de Zermatt; indiquée, avec doute, dans les cantons de Berne eldu Tessin, elle a été trouvée par M. Muret dans les Grisons. Habite Trieste, le Piémont, le Dauphiné, le Languedoc, la Provence et les Pyrénées. La Suisse est donc la station la plus septentrionale de cette espèce.

V. tenuifolia Roth. (V. Cracca L. var. tenuifolia Gaud. Cracca tenuifolia Gr. et. G.) Dans les haies de Martigny, Branson, Charrat. Se retrouve à Genève, et dans le canton de Vaud. Elle habite la France et l'Alle-. emagne.

* V. Onobrychioides L. Champs et buissons à Branson, Fouly, Saillon, Sion, etc. Habite l'Istrie, le Frioul, le Piémont, le midi de la France, les Cévennes et l'Au- 
vergne; outre ces locatités, N. Boissier l'indique en Espagne, en Italie, en Grèce et dans l'Afrique septentrionale. Elle manque en Angleterre et dans toute l'Allemagne du Nord. Le Valais serait done la station la plus septentrionale.

V. Lathyroides L. Cette petite plante n'existe en Suisse qu'à Sion, entre Valère et Tourbillon el à Peney, canton de Genève. Elle habite la France, la Corse, le Piémont, l'Allemagne et l'Angleterre.

Lathyrus sphæricus Retz. (L. coccineus All.) Celte plante n'a élé trouvée en Valais que près de Branson; elle croît en outre dans les cantons de Genève, de Lucerne et du Tessin. L'espèce habile le Tyrol, l'Istrie, le Piémont, la Corse et les provinces méridionales, centrales et occidentales de la France. Elle manque en Angleterre et dans tout le nord de l'Allemagne.

Obs. Le Lathyrus angulatus L. indiqué en Valais par Murith, Gaudin et Koch, n'a pas été trouvé et ce qu'en disent ces auteur's doit se rapporter à l'espèce précédente.

* Amygdalus communis L. naturalisé sur les rochers à Saillon, Contey, Sion, Saint-Léonard. Il est probable que l'Amandier est originaire de la Perse, de l'Asie Mineure: de la Syrie et de l'Algérie; c'est de là qu'il s'est naturalisé en Grèce, en Italie, en Sicile et dans le midi de la France où il est subspontané dans la région des oliviers $\in l$ cultivé dans la région des vignes. (A. D C. Georg. Bol.)

Potentilla caulescens L. Cetle jolie espèce habile les rochers de la Porte du Scex, et de St-Maurice et se retrouve entre Varonne et Inden, ancienne route des Bains de Loesche. Elle croît en outre au Mont-Pilate, dans les Grisons et quelques autres localités suisses. En France, 
elle habite le Jura, le Dauphiné, les Cévennes et les Pyrénées, les Alpes de l'Allemagne, les montagnes de l'Italie eh plusieurs localités de l'Espagne (Boissier). Elle manque en Angleterre et dans le nord de l'Europe.

P. rupestris L. Celte belle plante qui n'est pas commune en Suisse, se rencontre entre Salvan et Gueuroz, près de Martigny et dans la vallée de St-Nicolas en Valais; on la retrouve dans les cantons de Vaud, de Genève et du Tessin. Elle habite le centre et le midi de la France, la Corse, l'Espagne, le Piémont, l'Allemagne, l'Angleterre, la Suède et la Sibérie.

P. inclinata Will. Cette espèce rare indiquée à Branson, a été découverte par M. Haussknecht au-dessus des Marques. On la retrouve à Schaffhouse, Constance et dans le Tessin. En France, elle habite l'Alsace et le Dauphiné, les collines chaudes et sèches de l'Allemagne et les plaines du Piémont.

Obs. Gaudin a décrit, sous le nom de Potentilla parviflora, une plante qui croìt sur les collines du BasValais et en particulier aux Marques près de Martigny ; elle tient le milieu entre la P. intermedia L. et la P. inclinata Will., mais d'après le Prodrome, ce serait la $\mathrm{P}$. canescens Bess. C'est une plante à chercher et à examiner de nouveau.

Obs. La Potentilla cinerea Gaud. indiquée à Martigny, Branson, Fouly, Sion et ne parait pas être la plante de Chaix mais une variété remarquable de la $P$. verna $L$. se reconnaissant à ses feuilles velues et garnies de poils étoilés, c'est la var. psendo-cinerea Ducommun. L'espèce de Chaix a été trouvée à Bâle.

Punica granatum L. Indiqué par Haller, Murith et Gaudin sur les rochers de Valère à Sion, où il est subspontané; dès lors on l'a retrouvé „dans le Tessin. 
D’après Koch il serait naturalisé dans le Tyrol méridional et sur le littoral de l'Adriatique. Il paraît originaire de l'Asie occidentale d'où il s'est répandu en Grèce, en Afrique, en Espagne et dans le midi de la France. (A. D C. Geogr. Bot.)

Telephium Imperati L. Cette plante méridionale; rare pour la Suisse, ne se trouve qu'à Saillon, Contey, Sion et aux Plâtrières de Sierre : elle serait spéciale au Valais si elle n'avait pas été découverte près d'Ollon dans le canton de Vaud (Rapin). Elle n'est indiquée que dans le Tyrol méridional, le Piémont, le midi de la France, le Dauphiné et près d'Arbois dans le Jura. Elle manque en Angleterre et dans tout le nord de l'Europe. D'après M. Boissier elle habite en outre l'Espagne, la Georgie, l'Asie Mineure et l'Afrique septentrionale.

Opuntia vulgaris. Mill. (Cactus Opuntia L.) Cette plante étrangère s'est naturalisée à Sion, sur les rochers de Valère et au-dessus du Bouveret et de Vouvry; on la retrouve en Suisse dans le canton du Tessin. Elle croît en outre dans le Piémont, le Tyrol méridional. l'Italie, la Corse, la Grèce, la Sicile, le midi de la France et de l'Espagne. Elle est originaire du NouveauMonde d'où elle s'est répandue d'abord aux Canaries, puis en Afrique et finalement en Europe où elle se propage maintenant elle-même par graines. (DC. Geogr. Bot). La Suisse est la station la plus septentrionale de cette curieuse plante.

Bryonia alba L. Celte plante grimpante qui n'était connue en Suisse que dans le canton des Grisons, a été trouvée en Valais par M. Muret, près de Saxon, de Tourtemagne et de Viège. Beaucoup moins commune que la Bryone dioïque, elle habite quelques parties de l'Allemagne et parait manquer en France, car d'après 
MM. Grenier el Godron, c'est la dioica qui croît dans les localités françaises oủ l'alba avail été indiquée (Lorraine, Montauban, Pyrénées). Ils attribuent ces erreurs à ce que Linné a confondu les deux espèces sous le nom de B. alba. Celle-ci se distingue par ses baies noires, par ses fleurs femelles de moitié plus petites et ses stigmates glabres.

* Saxifraga exarata Vill. var. leucantha Gaud. (S. cœspitosa var. leucantha Gaud ) Cetle remarquable variélé croît sur les côteaux de Branson, de Fouly et sur les rochers du Trient en montant à Gueuroz; on ne la trouve pas ailleurs en Suisse. L'espèce type occupe toute la chaine des Alpes en Suisse, en Allemagne, en Piémont, en France, ainsi que les Pyrénées.

* S. bulbifera L. Cette rare espèce, spéciale au Bas-Valais, n'a été trouvée que près de Gueuroz et entre Branson et les Folateires; malheureusement cette dernière localité est presque détruile par les cultures. Elle habite l'Allemagne méridionale, le Piémont, l'Italie et la Corse; mais elle manque en France, en Angleterre et dans tout le Nord de l'Europe. Elle est rare dans toutes les stations où elle est indiquée. Linné qui n'avait reçu la plante que d'Italie penchait pour en faire une variélé de la S. granulata; mais les deux espèces sont parfaitement distinctes. La dernière est peu commune en Suisse, elle n'a élé signalée qu'aux environs de Bâle, dans une localité du canton de Vaud et près de Genève où elle est plus abondante.

* Pimpinella nigra Willd. Koch. P. Saxifraga L. var. nigra Gaud.) Marligny, Sierre, se trouvera probablement ailleurs. Elle diffère de la P. Saxifraga par l'indument des pédoncules, par ses tiges plus robustes et plus élevées et par cette particularité que sa racine, beaucoup plus épaisse, prend, lorsqu'elle est coupée, une 
teinte violette plus marquér dans les couches extérieures. D'après Koch elle croît sur les collines sèches du nord de l'Allemagne. Gaudin, Ducommun et Gremli sont les seuls auteurs suisses qui en fassent mention; encore ce dernier a-t-il des doutes sur la légitimité de l'espèce.

Buplevrum rotundifolium L. Plante des cultures qui se trouve dans les champs du Bas-Valais, à Montorge, Contey, St-Léonard, Sierre, etc. On l'a indiquée dans les moissons à Genève, Bâle et dans le canton de Vaud. Elle paraît spontanée, hors des cultures, autour du Caucase, et peut-être en Perse (DC. Geogr. Bot.). Du reste, elle habite les champs de l'Angleterre, de la France, de l'Allemagne, de l'Italie, etc. (Boissier).

Trochiscanthes nodiflorus lioch (Ligusticum nodiflorum Willd. Mur.) C'est une des rares espèces de la Suisse qui n'avait été signalée que dans le Bas-Valais entre St-Maurice et Martigny et au-dessus de Port-Valais (Murith, Gauãin). Mais elle a élé trouvée au-dessus d'Aigle, le long de la Grande-Eau par M. Haussknecht (Rapin). Habite le Piémont, l'Italie, les Alpes du Dauphiné et peut-être la Hongrie? Elle manque dans tout le reste de l'Europe. D'après le Prodrome elle croîtrait dans le canton du Tessin, mais aucun auteur suisse n'en fait mention.

Pencedanum Venetum Koch (Cervaria alsalica $\beta$ albiflora Gaud, Peucedanum alsaticum $\beta$ albiflorum D G.) Espèce peu commune, trouvée près de Pissevache par M. Muret, et indiquée dans lis vignes de Ravoire, près de Martigny par Murith et Gaudin; pour le reste de la Suisse, elle n'a été signalée que dans le canton du Tessin. Elle habite la vallée d'Aoste, le littoral de l'Adriatique et près du Pont-St-Esprit sur le Rhône, seule iocalité française. 
Orlaya grandiflora IIoffm. (Caucalis grandiflora L.) Cetle espèce, cultivée involontairement, se trouve dans les champs de Branson et de Lidde. Elle croît dans les moissons des cantons de Genève, de Vaud, de Bâle et de Zurich. Du reste, e!le habite la France, l'Allemagne, l'Espagne, l'ltalie, la Grèce et la Taurie.

* Turgenia latifolia Hoffm. (Caucalis latifolia L.) Comme la précédente, cette espèce ne se trouve que dans les cultures. Pour la Suisse, elle est spéciale au Bas-Yalais et n'a été récoltée que dans les champs près de Saillon, de Contey et de St-Severin. Elle paraît sauvage dans les montagnes du Suvant, et peut-être en Sibérie. (D G. Geogrr. Bot.) Elle habite l'Angleterre, la France, l'Espagne, l'Allemagne méridionale, la Taurie, l'Asie Mineure, la Perse, l'Afrique septentrionale (Boissier.)

* Anthriscus cerefolium IIoff. $\beta$ trichospermum DC. Prodr. (A. trichosperma Schults, Koch.) Cette variélé remarquable, qui n’a été signalée ni par. Murith, ni par Gaudin, se trouve en abondance au milieu des rochers et des buissons à Valère et à Tourbillon. Elle habite la Bıhême, la Honģrie el l'Autriche. D'après quelques auteurs ce serait le type sauvage du Cerfeuil cultivé qui se répand souvent autour des habitations. Cependant, il en diffère en ce que notre plante est plus haute et plus diffuse que le Cerfeuil, et que les fruits sont hispides et du double plus gros.

Cornus mas L. Le Cornouiller n'est pas rare sur les côteaux du Bas-Valais; il est abondant au Bois-Noir, près de St-Maurice. M. Papin indique plusieurs localités du canton de Vaud. Il habite l'Allemagne méridionale, le Piémont, la France, et manque en Angleterre et dans le nord de l'Europe. Il est fréquemment cultivé.

* Lonicera Etrusca, Sant. Celte espèce rare, indi- 
quée par Schleicher, en Valais, sans désignation de localité, a été trouvée par M. Muret, entre Fouly et Saillon, seule localité Suisse. Elle habite le Piémont, le littoral de l'Adriatique, la France méridionale jusqu'en Auvergne, l'Italie et l'Espagne (Boissier). Se distingue du L. Caprifolium L. par ses feuilles plus grandes, pubescentes en dessous, et dont les inférieures sont plus longuement pétiolées, par ses capitules terminaux pédonculés, etc.

* Asperula longiflora W. et K., Koch. (A. flaccida Rap. (A. cynanchica $\beta$ longiflora Reich.) Collines sèches du Bas-Valais, Martigny, Branson, St-Léonard, etc... L'espèce habite le midi de l'Allemagne, le littoral de l'Adriatique, l'Isère, le Dauphiné et le Var en France. Elle diffère de l'A. Cynanchica L. par ses corolles glabres et lisse et par le tube de la corolle qui égale trois fois la longueur du limbe (Grenier.)

Obs. l'Asperula flaccida Ten. ne se trouverait pas en Valais, mais seulement dans le Tessin (Gremli).

Galium Pedemontanum All. Gette rare espèce n'était connue en Suisse qu'à Fouly et à Branson, oủ elle était peu abondante; M. Rapin en a récolté de superbes échantillons, en assez grande quantité, en montant à Joux-Brûlée. M. Muret l'a retrouvée dans le Tessin, à Orsolina et à Cadro (Gremli.) Elle habite la Valteline, le Piérnont, le littoral de l'Adriatique, l'Autriche, la Hongrie, l'Espagne et le Portugal. Manque en France, en Angleterre et dans le nord de l'Europe.

Rubia tinctorium L. La Garance existe à la Porte du Scex, à Contey, à Sion et à Sierre, ainsi que dans le canton de Vaud et dans celui de Berne; cultivée dans le midi de la France, elle se retrouve spontanée un peu partout. La Garance est sauvage en Grèce et en Italie, et c'est de là qu'elle s'est répandue 
dans le reste dzl'Europe. La culture de cette plante est très-ancienne, les Romains la connaissaient, et en France les actes du moyen àge en font mention. Elle a été cultivée en grand en Hollande, en Allemagne, en Grèce, en Asie Nlineure et en Syrie (D C. Geogr. Bot.) Il est probable qu'on a fait également des essais en Valais.

Chrysocoma Linosyris L. (Linosyris vulgaris Cass.) Sur les collines arides du Bas-Valais, Martigny, Sierre, etc. Se trouve dans les cantons de Vaud, de Neuchàtel, de Schaffouse et des Grisons. Elle habite le midi de l'Allemagne, presque toute la France, l'Angleterre, l'Espagne et la Taurie.

Micropus erectus L. Cette espèce méridionale se trouve à Hontorge, Sierre et probablement ailleurs, elle existe en outre dans les cantons de Vaud et degenève. Elle croît sur le littoral de l'Adriatique, sur les côteaux arides de presque toute la France, en Espagne, en Italie, en Asie Mineure, en Perse et dans le nord de l'Afrique (Boissier.)

* Artemisia Valesiaca All. Gaud. D G. Prodr. (A. maritima $\delta$ valesiaca Koch.) Cette belle espèce ne se trouve en Suisse que sur les coleaux du Valais, à Fouly, Saillon, Contey, Montorge, St-Léonard, Sierre, Varone, etc. En dehors de nos limites, elle se retrouve dans le Tyrol, et en Piémont, dans le Val d'Aost.

Koch admet quatre variétés de l'A. maritima L. savoir : $\alpha$ maritima Willd. $\beta$ gallica Willd. $\gamma$ salina Willd. et $\delta$ valesiacia All. Cette dernière se distingue par l'indument blanc qui la recouvre en entier, et par ses capitules el ses rameaux dressés. Le Prodrome de De Candolle admet, au contraire, quatre espèces 
distinctes, l'A. maritima L. qui croît sur les côtes maritimes du nord de la France, de l'Angleterre, de la Suède, du Danernark, etc., l'A. gallica Willd. qui croît dans les sables maritimes de la France méridionale et de l'Espagne, l'A. salina Willd, qui habite autour des salines de l'Allemagne, et l'A. valesiaca All. qui croît dans le Bas-Valais et en Piémont. NIM. Grenier et Godron décrivent, comme espéces distinctes, l'A. maritima L. et l'A. gallica Willd.

* Achillea tomentosa L. Cette plante méridionale croit à Branson, Fouly, Sierre, et dans la vallée de Saas. On la retrouve en Piémont, dans le Tyrol méridional, en Italie, dans le midi de la France et en Dauphiné. On l'indique en Ecosse et en Irlande, mais plutòt naturalisée que spontanée. Elle habite encore la Sicile, l'Espagne, l'Afrique septentrionale; c'est une des plantes méridionales spéciales au Valais.

* A. setacea W. et K. Gaud. Rap. Grem. (A. millefolium var. setacea Koch). Spéciale au Bas-Valais, cette plante croît sur les coteaux de Charrat, Saxon, Fouly, Montorge, Sierre. Elle se distingue de l'A. millefolium L. par son port et son odeur, sa villosité plus grande, ses capitules plns pelits, sa taille moins élevée, et par les lanic̀res de ses feuilles très-étroites. Elle habite les provinces méridionales de la France, les Pyrénées, les Alpes, la Thuringe, la Hongrie, la Perse, la Sibérie altaïque, etc.

A. nobilis L. se trouve dans les mêmes localités que la précédente, mais elle est plus commune; elle est indiquée par M. Godet, à Chaumont près de Neuchâtel. Elle habite l'Allemagne, la France, l'Italie et peut-être l'Espagne.

* Echinops sphærocephalus L. croît çà et là ả Bran- 
son, Sion, Sierre et Brigg dans le Haut-Valais et n'est pas signalé ailleurs en Suisse. Habite le Piémont, la France, l'Espagne, l'Allemagne, la Transylvanie, la Taurie et la Sibérie.

* Crupina vulgaris Cass. Pers. (Centaurea Crupina L.) Elle végète dans les maigres cultures de Branson, Fouly, Montorge, Saint-Léonard, Sierre et pas ailleur's en Suisse. Elle habite le Piémont, la Hongrie, l'Istrie, la France méridionale d'oú elle remonte du côté de l'Ouest, le nord de l'Afrique, les îles de la wed et en Orient, la Perse, etc. Le Valais est la station la plus septentrionale de celte espèce.

* Xeranthemum inapertum Willd. (X. annuum $\beta$ inapertum L. X. erectum Presl. DC. Prodr.) Cette plante, spéciale pour la Suisse au Bas-Valais, n'y est pas rare; on la trouve à Martigny, Saillon, Montorge, Sion, SaintLéonard, Sierre, Losche-la-Ville, etc. Elle habite le Piémont, l'Italie du nord, le midi et le centre de la France, l'Espagne, la Sicile, la Grèce, le Caucase, la Syrie el la Perse. (Boissier). Elle manque en Allemagne, en Angleterre et dans le nord de l'Europe. Le Valais est encore la station la plus septentrionale de celte plante.

* Centaurea valesiaca Jord. Rap. Greml. Ducom. (G. paniculata L. $\varepsilon$ valesiaca DG. Prodr., C. paniculata Murith, Gaud., Moritz.) Cette plante croît en abondance à Montorge, Sion, Saint-Léonard, Sierre, etc., ę ne parait pas exister ailleurs en Suisse. Sous la dénominalion de G. paniculata, Linné a confondu plusieurs espèces qui ont été distinguées sous les noms de $\mathrm{C}$. valesiaca Jord., abondante en Valais, C. Mureti Jord., qui n'a été signalée que dans les Grisons, C. maculosa Lam. qui croît aux environs de Bâle et G. paniculata L. qui, pour 
la Suisse, n'était connue que dans une seule localité du canton de Vaud, près de Nyon où j'en ai retrouvé un certain nombre. Cette dernière, est une plante méridio-

- nale qui habite le midi de la France, l'Espagne et l'Italie. (DG. Prodr.). La C. paniculata Lam. non L. croît seulement en Valais, d'après Koch et doit se rapporter à notre espèce qui diffère de la plante de Linné par ses rameaux moins étalés, ses capitules une fois plus gros, les cinq nervures très-saillantes de l'involucre dont l'appendice est large, brun, peu acuminé (Rapin). Caractères qui se voient très-bien en comparant les deux plantes vivantes qui ont également un aspect différent.

Chondrilla juncea $L$. avec la var. $\beta$ rigens du Prodr. Assez abondante sur les coteaux secs du Bas-Valais, Martigny, Branson, Fouly, Sion, Sierre, etc. Pour le reste de la Suisse, se retrouve à Genève, à Bâle, dans le canton de Vaud, au Tessin, etc. Habite l'Allemagne, la plus grande partie de la France, le Piémont, l'Espagne et la Sibérie. Manque en Angleterre.

Lactuca virosa L. croît à Branson, Sierre et entre Viège et Stalden; se retrouve dans les cantons de Genève, de Vaud et de Neuchâtel. Habite l'Allemagne, oủ elle est plutôt rare, une partie de la France, surtout le Midi et le Centre, le Piẻmont, l'Angleterre et généralement l'Europe méridionale,

L. Scariola L. (L. sylvestris Lam.) Croît à Martigny, Branson, Saxon, Sierre, Viège, elc.; se retrouve dans les cantons de Vaud, de Genève, de Bâle. Cette plante habite presque toute l'Europe, la Suède méridionale, l'Angleterre, la Hollande, l'Allemagne, la Sibérie, la France, le Piémont, l'Espagne, l'île de Madère, l'Egypte, l'Arabie, etc. Au midi du Caucase elle a une apparence plus sauvage et plus primitive, aussi ce serait possible 
(qu'elle fût la souche de la laitue cultivée. (DG. Geogr. Bot.)

* L. Augustana All. Prode. (L. scariola L. $\beta$ integrata Gr. et G.) Se trouve a Sierre, Varonne, Viège, etc.; elle habite le Piémont (Vallée d'Aoste), la Perse, l'Egypte et probabicment bien d'autres localités, car la plupart des auteurs n'en font qu'une variélé de la précédente. Quoiqu'il en soit, elle se reconnaît à sa taille élevée; elle est très-glabre, lactescente et sans odeur. vireuse; les feuilles supérieures sont entières, les côtes et les nervures sont dépourvues d'aiguillons.

L. perennis L. croît à Saint-llaurice, à Branson, Martigny etc., dans le canton de Vaud. près de Genève, à Bàle. Habile l'Allemagne du Sud, le Piémont, la France; manque en Angleterre et dans le nord de l'Europe.

* L. viminea Link. Koch. (Prenanthes viminea L. Phœnixopus vimineus Reich. Pren. ramosissima Gaud. Phœnopus vimineus DC. Prodr.) Se trouve à Martigny, Branson, Mlontorge, Sion, Sierre, Saint-Nicolas. Elle n'est pas signalée ailleur's pour la Suisse. Elle habite l'Autriche, la Bohème, le Piémont, le midi de la France, région des oliviers, l'Espagne, la Grèce el la Taurie.

Hieracium lanatum Vill., non Willd nec W. et Kit. (Andryala lanata L. II. tomentosum All.) Celte espèce rare vit sur les rochers entre Charrat et Saxon, à Saillon $t$ t au-dessus de Varonne en allant à Inden. On l'indique dans le canton de Neuchàtel et près de Genève au Salève et au Vuache. Elle habite en outre le Piémont, la Savoir, les Alpes du Dauphiné. Manque dans le reste de l'Europe.

H. pictum Sch!. (H. andryaloides Vill. $\gamma$ pictum Koch.?) 
Celte espèce peu commune, se trouve à Saint-Maurice, Charrat, Sion, Sierre, le long de la route du Simplon et au canton de Vaud. Les feuilles radicales sont oblongues, lancéolées, sinuées, dentées, garnies sur le péliole et sur leur face inférieure de longs poils laineux et plumeux, glabres et maculées de violet en-dessus, l'involucre est canescent. (Rapin.)

H. Valesiacum Fries. Peu connu, je l'ai récolté entre Sierre et Varone et M. Rapin en montant à Salvan; il est aussi indiqué dans le canton de Vaud. Voici la description qu'en donne M. Gremli el dont je dois la traduction à l'obligeance de mon ami Bernet. "Tige entièrement feuillée, fortement poilue; feuilles à bords entiers ou finement dentelés, glanduleux, elles sont acuminées, couvertes des deux côtés de poils blancs et roides, les inférieures sont lancéolées, les autres ovalesembrassantes; les pédoncules et les involucres sont couverts d'un duvet étoilé, avec des poils simples, blancs à leur extrémité, entremêlés de poils glanduleux. Les dents de la corolle sont faiblement ciliées. Pistil d'un brun-noirâtre, fruits d'un brun-rougeâtre. M. Gremli le classe dans la division des Accipitrina et le groupe des Prenanthoidea.

Tragopogon major Jacq. N'est pas rare dans les champs incultes, le long des chemins, à Martigny, Branson, Charral, Sion, Bramois, Saint-Léonard, etc. Indiqué dans les cantons de Bâle et d'Argovie, il a élé découvert par M. Muret dans la Basse-Engadine. Il habite l'Allemagne, oủ il est peu commun, le centre, l'ouest et le midi de la France; n'est pas mentionné par Allioni. Manque en Angleterre et dans le nord de l'Europe. On le trouve en Asie.

Scorzonera austriaca Willd, (S. angustifolia, Reich. 
S. humilis Jacq. L. $\beta$ austriaca DG. Prodr.) En Valais elle existe sur les rochers et les prairies rocailleuses, à Saint-Maurice, Branson, Saxon, Fouly, etc.; dans le canton de Vaud à Olon, Aigle et Bex. Elle a été indiquée par Gessner sur l'Albis, mais est-ce bien la même espèce? Du reste, elle habite le Tyrol, l'Autriche méridionale, le littoral de l'Adriatique, le Piémont, le centre de la France, le Dauphiné, l'Ardèche. D'après le Prodrome elle se trouve en Sibérie.

Obs. La Scorzonera humilis L. Willd. (S. plantaginea et macrorhiza Schl. Gaud.) croît dans les prairies humides et tourbeuses de quelques localités de la Suisse (Vaud, Zurich), de l'Allemagne, de la France, etc., tandis que la S. austriaca Willd. se trouve toujours sur les rochers ou sur les prairies rocailleuses exposées au soleil.

Podospermum laciniatum DC. (P. muricatum Gaud. Scorzonera laciniata L.) Fréquent dans les champs et le long des chemins du Bas-Valais, il était spécial à ce cantun, mais il paraît qu'on l'a découvert dans celui du Tessin. Il habite une partie de l'Allemagne, le Piémont, presque toute la France, l'Espagne, l'Italie et la Grèce. Ianque en Angleterre et dans le nord de l'Europe.

Xanthium strumarium L. Cette espèce rudérale est rare dans le Bas-Valais où elle n'a été signalée qu'à Branson, Martigny, Sion et Saint-Léonard. On la trouve çà et là dans le canton de Vaud, dans le canton de Bâle et aux environs de Genève; elle est dispersée en Allemagne, commune en Piémont et en France, se retrouve en Angleterre et en Hollande oủ elle est sur sa limite de naturalisation, elle existe aussi en Espagne. D'après M. Boissier, elle habite presque toute l'Europe depuis la Sibérie et la Suède méridionale jus- 
que dans le nord de l'Afrique. Elle a été introduite en Amérique où elle s'est naturalisée.

Campanula bononiensis L. (C. Thaliana Wallr. C. simplex DC.) Celte rare espèce existe à Branson, Fouly, Conthey, ainsi qu'entre Martigny et Saint-Brancher. D'aprês Gaudin, elle élait spéciaie au Bas-Valais, mais il parait qu'on l'a signalée dans le canton du Tessin. En France elle ne croit que dans le Dauphiné, le Var et aux environs de Gap; elle habite en outre l'Allemagne du Sud, le Piejmont, la Sibérie et le Caucase. Elle manque en Angleterre et dans le nord de l'Europe.

C. spicata L. Elle croît ả Martigny, Fouly, Montorge et Sion et se retrouve dans le Tessin. Elle habite les collines rocailleuses de l'Allemagne méridionale; en France, le Dauphiné et les Basses-Alpes; le Piémont, oú elle n'est pas rare; mais elle manque en Angleterre et dans le nord de l'Europe. Linné dans son Species ne l'indique qu'en Valais.

* Pyrola arenaria Rap. (P. rolundifolia var. arénaria Koch. P. media Thom. non Sw.) Cette espèce dé-

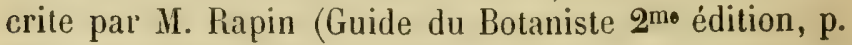
83), n’a été trouvée jusquà présent que dans la vallée de Bagnes, non loin de Martigny. Koch l'indique in Insula Nordeney. Grenier et Gudron admeltent la var. arenaria de Koch et la signalent sur les dunes de SaintQuentin et à l'embouchure de la Somme. Elle diffère de la Pyrola rotundifolia L. par ses feuilles ovales-elliptitiques de moitié plus petiles, par sa grappe de fleurs de moitié plus courte et par les divisions du calice linéaires-oblongues, obtuses et plus larges.

Onosma stellulatum W. et K. (0. montanum Gaud. 0. echioides Rap. Ducom. 0. Helvetica Boiss. Diagn. plant. $\mathrm{n}^{0} 11, \mathrm{p} .111$. 0 . echioides var. helvelica DG. 


\section{7}

Prodr. in nota). Cetle espèce crilique croît sur les coteaux arides des Marques, de la Bâtia, de Branson, de Fouly, de Vetroz, de Sierre, elc. On la retrouve dans le canton de Vaud, district d'Aigle, avec l'Onosma echioides L. Gaud. (0. vaudense Gremli.) qui habite la colline ypseuse de Tombey près d'Olon, mais qui manque en Valais.

Notre Onosma a la tige simple ou bifurquée, trèsrarement trifurquée, hérissée de poils raides ef piquants insérés sur de petits tubercules entourés de poils rayonnants; elle a les filets des étamines plus longs que l'anthère et la corolle deux fois plus longue que le calice. L'O. Helvetica Boiss. ne diffère de la plante du Valais que par les poils des feuilles inférieures insérés sur un tubercule glabre, dépourvu de poils rayonnés, tandis que les soies des autres parties reposent sur des tubercules à poils étoilés.

L'espèce habite le Tyrol méridional, Trieste, la Savoie, le Piémont et le Dauphiné. (Reuter.)

Gaudin, dans sa flore helvétique, n'avait décrit d'abord qu'une seule espèce, sous le nom d'Onosma montanum Sm. (0. stellulatum W. et K.), croissant au canton de Vaud et surtout en Valais. Dans un appendice, il décrit une seconde espèce sous le nom d'Onosma echioides Sm. et qui se rapporte à la plante de Linné. Elle ne se trouve en Suisse qu'au Tombey, près d'Olon, ne croit pas en Valais et est identique à l'espèce observée en France. (Reuter).

Dans une note, Gaudin fait remarquer que l'0. stellulatum W. et K. ne paraît pas différer de son 0. montanum, c'est aussi l'opinion de M. Boissier, qui pense que ces deux plantes doivent être réunies.

Gaudin ajoute que Linné avait, probablement avec 
raison, regardé ces deux formes comme deux variétés d'une même espèce. Voici du reste leur description:

Onosma stellulatum W. et K. (0. montanum Gaud.). Racine ligneuse, rougeâtre extérieurement. Tiges de 30 à 40 centimètres, dressées, simples ou bifurquées au sommet, hérissées de poils longs, raides, piquants, rousseatres à la partie supérieure. Feuilles sessiles, longues, linéaires-lancéolées, obtuses, très-rudes, planes, recouvertes de chaque côlé de tubercules qui portent un long poil solitaire et qui sont entourés à leur base de petits poils courts, appliqués et rayonnants. Pédoncules hérissés el très-courts. Les cinq divisions du calice sont allongẻes, aiguës et très-velues. La corolle est d'un blanc jaunâtre, tuberculeuse, penchée, deux fois plus longue que le calice. Anthères allongées, dépassant le filet ou l'égalant en longueur.

0. Helvetica Boiss. (0. echioides L. var. Helvetica D G. Prodr. in nota). Caulibus cœespitosis, erectis, basi induratis, foliosis, selis albis patulis e tuberculo piloso ortis, strigosis, simplicibus ; foliis lanceolalo-linearibus, obtusis margine sub revolutis, setulis adpressis ad folia inferiora et ad basin foliorum superiorum e tuberculo glabro oriundis, ad folia superiora e tuberculo stellatim piloso ortis asperis ; ramo bifido, divaricalo, terminali; calicis adpresse flavido-setosi laciniis lanceolatis, acutis; corollà pallide ochroleucâ, puberulâ, calyce subduplo longiori, lobis triangularibus brevibus; filamentis latis, parte liberâ antheræ aequilongis; nucibus læviusculis.

Species cum quâdam dubitatione hic proposita, inter 0 . echioidem et 0 . stellulatam media, et eos naturâ indumenti conjungens, ulterius observanda. (Boissier. Diagnoses plantarum orientalium novarum, $\mathrm{n}^{\circ}$ 11.)

Onosma echioides. L. Tiges rameuses, plus élevées, diffuses, hérissées de poils plus raides. Feuilles linéaires- 
lancéolées, bispides, planes, dépourvues de poils rayonnants. Les tubercules qui dornent naissance aux poils sont glabres, mais rugueux. La corolle est penchée, sub-cylindrique, une fois plus longue que le calice. Les anthères sont presque de la longueur du filet.

Obs. L'O. echioides L. du canton de Vaud est identique avec la plante de France, d'Allemagne el de Hongrie. La tige est toujours rameuse, les poils sont plus fins, plus appliqués et leur tubercule basilaire est toujour's dépourvu de poils rayonnants. Reichenbach indique en outre dans les carpelles un caractère à vérifier sur a plante vivante et qui la distinguerait de l'0. stelldatum. (Reuter.)

M. Rapn (Guide du Botaniste) n'admet qu'une seule espèce el réunissant sous le nom de 0 . echioides $L$. la plantedu cailton de Vand el celle du Valais.

La pluart des auteurs suisses en reconnaissent deux, mais il re sont pas d'accord sur les noms, et récemment M.Gremli (Beitrage zur Flora der Schweiz 1870) admet l'). stellulatum en Vạais et décrit comme espèce nouvelle sous le nom d'O. vaudense la plante du canton de aud.

- Mosotis stricta Link. (M. collina $\beta$. stricta Gaud, I. arenaria Schr. M. arvensis Reich.) On ne l'a trouvé usqu'à présent que sur la colline de Branson, dans ls champs incultes et dans les vignes. C'est la seule lcalité suisse. L'espèce habite la Suède, l'Allemagnc la Suisse, la France et l'Espagne (Boissier). Il se disngue du M. collina Ehr. par ses pédoncules extrêrement courts, par sa corolle très-petite el par ses caces fructilères dont les lobes sont fermés.

- Ephrasia viscosa L. (Odontites viscosa Lam). Cette espèc rare ne se trouve que dans la forêt de Finges où eb est peu abondante et au-dessus de Varonne le 
long de l'ancien sentier qui conduisait à Lœsche-lesBains oủ elle croît sous les pins, mélangée à l'E. lutea $L$. On l'a aussi indiquée près de Branson? Spéciale au Valais, elle ne se retrouve qu'en Piémont, au Midi de la France, en Espagne, en Porlugal et au nord de l'Afrique. (Boissier.)

Veronica prostrata L. Cette jolie espèce se rencontre à St-Maurice, Marligny, Branson, Gharrat, Sierre, etc. Elle est indiquée à Bâle par Gaudin et à Sshaffouse par Moritzi; cependant Gremli dit qu'elle ne se trouve qu'en Valais. Elle est répandue dans le Sud.Ouest de l'Allemagne, signalée en France sans désigration de localités, eile croît en Piémont et manque in Anglegleterre et dans le Nord de l'Europe.

Rosmarinus officinalis L. Le romarin s'es naturalisé à Sion sur les rochers de Valère et de Torrbillon; on le trouve également dans le district d'Aiglt et dans les environs de Montreux. C'est une plante néridionale qui habite l'Espagne, le Midi de la France le Piémont, la Corse, les bords de l'Adriatique, l'ssie mineure et l'Afrique septentrionale.

Hyssopus officinalis L. Cette petite plante cDit sur les coteaux de Branson et des Folateires, de Mrtigny, etc, Elle se retrouve dans le Tessin. Du reste lle habite l'Allemagne méridionale, le Piémont, le Sul de la France, l'Espagne, l'Asie et la Perse. Elle estplutôt naturalisée que sponianée dans le centre et le Nrd de la France ainsi qu'en Angleterre. Elle a été intrduite en Amérique.

Origanum vulgare $L$. $\beta$ prismaticum Gaud. (C vulgare $\beta$ megastachyum Koch. 0 . crelicum L. SutDC.) Cette remarquable variété croit à Martigny, Sierr, etc. on la rencontre rarement dans le Canton de Vaucet il 
il m'a paru qu'elle est plus fréquente que le type dans le Bas-Valais. Elle existe dans l'Allemagne du Sud et le midi de la France.

Salvia officinalis L. La Sauge paraît spontanée à Fouly, Valère et Tourbillon. Elle se retrouve dans le Tessin. L'espèce habite le littoral de l'Adriatique, le Piémont, les Pyrénées orientales, la Provence et la région des oliviers en France, la Corse et l'Espagne. D'après M. Boissier la var. Hispanica qu'il a décrite serait le type sauvage de la $\mathrm{S}$. officinalis.

S. Sclarea L. Celte belle espèce n'est pas commune dans le Bas-Valais où elle existe à Fouly, Contey, Sierre, etc. ainsi que dans quelques localités du Canton de Vaud. On la trouve dans l'Allemagne du sud, en Piémont, dans le midi de la'France, en Syrie et en Arabie. On la cultivait autrefois dans les jardins d'où elle s'échappait souvent.

Dracocephalum austriacum L. Cette rare et belle plante, dont Schleicher cachait soigneusement la station, avai: étẻ retrouvée par 1I. Muret près de Dorénaz, dans le Bas-Valais, sur des corniches escarpées au-dessus du Rhône. Malheureusement la Iocalité a été detruite quand on a refait le sentier, mais on pourrait la retrouver aux environs. Elle a été découverte par $\mathbf{M}$. Coatz dans la Basse-Engadine oủ M. Muret l'a récoltée en 1868. (Gremli.) Pour l'Allemagne elle n'est indiquée qu'en Bohème, en Gallicie et en Autriche. En France elle habite les Alpes du Dauphiné, la Frovence et les Pyrénées orientales. Je ne crois pas qu'elle aît été signalée ailleurs.

Primula viscosa Vill. (P. villosa Jacq.) Les touffes de celte jolie primevère à fleurs purpurines couvrent les rochers du Trient près de la Gorge, ainsi qu'en 
montant à Gueuroz et avant d'arriver à Salvan. Fille habite surtout les Alpes du Canton de Vaud, du Valais, de Glaris, d'Uri, etc. En Allemagne on la trouve dans le Tyrol et lat Carinthie, en Piémont, en France sur les Alpes du Dauphiné et sur les Pyrénées.

- Androsace maxima L. Ceite espèce se trouve dans les moissons du Bas-Valais à Montorge, Aven, St-Léonard, Sierre; elle ne croît pas ailleurs en Suisse. Elle habite l'Allemagne, le Piémont, la Frănce, l'Espagne, l'Italie, la Syrie, la Perse, et la Sibérie. (Boissier) Elle manque en Angleterre, ainsi que toutes les espèces du genre.

Cyclamen Neapolitanum Ten., Gremli, Ducom. (C. hederaefolium Gaud. Rap. Koch. non Ait.) On l'a trouvé au pied des rochers de la porte du Scex. Il existe près de Roche dans le Canton de Vaud, dans le Tessin et les Grisons; Koch ne le signale qu'en Suisse, il habite le midi et Ie centre de la France, la Savoie ainsi que l'Italie. Les fleurs paraissent en automne avant le déveveloppement des feuilles, elles sont inodores, d'un blanc rosé et la gorge de la corolle est marquée de saillies d'un rouge vif; les Iubercules ont la forme d'un disque aplati. Le C. hederæfolium Ait. fleurit au printemps, il existe en France, en Corse. en Angleterre, etc.

Obs. Le C. Europæum L. a été indiqué par Murith dans le Bas-Valais.

Laurus nobilis L. Cultivé dans les jardins, il parait sub-spontané à Vouvry et à Sion, ainsi qu'aux environs de Montreux. Originaire de l'Orient, le Laurier des poètes s'est propagé et naturalisé graduellement en Grèce, en Ilalie et dans le midi de la France.

Ficus Carica L. Le figuier est sub-spontané à Martigny, Saillon, Contey et Sion. Les espèces cultivées paraissent originaires de l'Asie occidentale, de la côte 
septentrionale de l'Afrique et peut-être du sud-est de l'Europe. (D C. Geogr. Bol.)

* Ephedra Helvetica C. A. Mey. Greml. Ducom. (E. distachya Murith, Gaud. Rap. Koch non L.) Cette singulière plante, spéciale à la Suisse, ne se trouve que dans le Bas-Valais sur les rochers de Branson, Fouly, Saillon, Montorge, Tourbillon et Valère. Cette espèce se distingue par ses fleurs mâles grandes, ses gros glomérules, ses tiges à branches couchées; par ses rameaux d'un vert glauque, épais, fortement striés, très-rugueux; par les gaines des nœuds à tube aussi long que large, non évasé, à lobes ovales, obtus. L'E. distachya L. habite le Tyrol méridional, le Piémont et les coteaux maritimes des bords de la Méditérannée el de l'0céan.

Juniperus Sabina L. Rare dans le Bas-Valais, la Sabine n'est indiquée que près de la cascade de Pissevache et à Martigny; elle est plus abondante dans le Haut-Valais et en particulier dans la vallée de Zermatt; on la trouve çà et là en Suisse dans les Alpes. Elle habite l'Allemagne du Sud, le Piémont, le Dauphiné, les Pyrénées, l'Espagne, l'Italie, la Grèce, l'Asie Mineure et la Sibérie. (Boissier.)

* Iris virescens. DG. Rap. Greml. Ducom. (I. lutescens Mur. Gaud., Moritz. non Lam.) Cette espéce rare, spéciale au Bas-Valais ne se trouve qu'à Sion sur les rochers de Tourbillon; elle est indiquée avec doute à Martigny et dans le Tyrol méridional, mais elle paraît manquer en France. D'aprìs MIM. Grenier et Godron on a confondu plusieurs espèces sous le nom d'Iris lutescens qui a été donné par Gaudin et Reichenbach à une plante décrite par Redouté sous le nom d'Iris virescens.

Crocus sativas All. (C. autumnalis Schr. Murith). Il 
a été cultivé jadis en Valais et j'en ai trouvé sur les rochers de Montorge des échantillons sub-spontanés. Le Safran est sauvage en Grèce et vraisemblablement en Perse et en Asie Mineure où il est cultivé de toute ancienneté (DC. Geogr. Bot.) Il s'est naturalisé dans quelques localités du Tyrol méridional, du Piémont, de la Savoie et de la France.

Asparagus officinalis L. L'asperge se trouve à l'état sauvage sur les coteaux à Martigny, Branson, Sion, Sierre, etc.; elle croît également dans les Grisons. On la rencontre dans quelques localités de l'Allemagne, un peu partout en France, en Angleterre, etc. Originaire d'Europe elle s'est naturalisée aux Etats-Unis.

Ruscus aculeatus L. Rare en Valais, le petit Houx n'a élé trouvé que près de la cascade de Pissevache et et pour le reste de la Suisse dans le Canton de Vaud et dans le Tessin. Il croît dans l'Allemagne du sud, dans les lieux stériles d'une grande partie de la France, en Piémont, en Anglelerre, en Espagne, en Grèce, en Afrique; mais il manque dans le nord de l'Europe.

* Bulbocodium vernum L. Cette plante méridionale, rare et spéciale au Bas-Valais se trouve à Mivellaz au bord du Rhône, à Branson, à Fouly, à Sion, aux Agettes (Mayens de Sion, MM. Muret et Micheli) à Montorge, St-Léonard, à fleı!rs blanches (Murel). Elle n'est indiquée qu'en Ilongrie, dans les Alpes du Dauphiné, à Nice et dans les Pyrénées.

* Tulipa Didieri. Jord. Gren. el God. (T. Oculus solis Murith. Gaud. Koch non St-Am. T. maleolens Reich. Greml. Ducom.) Cette belle espèce ne se trouve que dans des champs près de Sion, localité unique pour la Suisse. Elle se distingue de la T. Oculus solis par la grandeur de ses stigmates qui dépassent le diamètre de 
de l'ovaire, par la forme et la couleur de sa fleur et par la tunique du bulbe qui n'est pas laineuse, mais seulement un peu poilue en dedans. En France, elle croit aux environs de Guillestre et dans la Maurienne. L'Oculus-Solis habite la Provence, le Languedoc, le Bassin de la Garonne etc., il semblerait même qu'elle ne s'est naturalisée à Montpellier que depuis l'époque de Magnol. (A. D C. Geog. Bot.)

* Gagea saxatilis Koch. Hegestsch. Rap. (G. arvensis var. subuniflora Reich. Ornithogalum minimum Nurith. 0. Bohemicum Gaud. non Lamarck.) Cette petite miniature est rare en Suisse, car on ne la trouve que dans le Bas-Valais, encore y est-elle peu fréquente.

Elle avait été signalée par Nurith et Gaudin sur les pelouses de Branson et je l'ai retrouvée, il y a quelques années au pied des rochers de Valère, à Sion. Elle est signalée dans quelques localités de l'Allemagne; mais elle paraît manquer en France, en Angleterre et dans le reste de l'Europe.

Obs. J'ai trouvé le long des sentiers qui conduisent de Branson aux Folateires quelques échantillons de la G. arvensis Sch. uniflores et bien plus petits que ceux que l'on rencontre en général dans les champs; on les reconnaît cependant à leur tige glabre, nue, dépourvue de feuilles et à l'ovaire plus échancré au sommet.

Sparganium minimum Fries. S. natans auctor. non L. S. natans $\beta$ minimum Gaud.) Trouvé par M. Reuter dans les fossés des marais de Vouvry. Celte espèce paraît remplacer en Suisse le vrai S. natans L. dont elle se distingue par son style court, par la brièveté du pédicèle et par ses feuilles d'un vert pâle. Elle existe dans quelques marais du Canton de Vand, de Zurich, dans la vallée du Rhin. En France elle habite l'esı, le centre, l'ouest et les Pyrénées. 
Tragus racemosus Desf. Hall. (Cenchrus racemosus L. Lappago racemosa Schr. Host.) Il n'est pas rare sur les collines de Branson, Fouly, Gharrat, Montorge, Sion, St. Léonard, etc., et serait spécial au BasValais s'il n'avait pas été trouvé à Bex, Canton de Vaud. Il habite la Belgique, la Dalmatie!, l'Autriche, le Tyrol méridional, le Piémont, le sud-ouest de la France, l'Espagne, le nord de l'Afrique et l'Amérique méridionale. (Boissier.)

Phleum asperum Vill. (Phalaris aspera Willd.) Se trouve çà et là dans les cultures el les vignes à Martigny, Montorge, Sion et dans quelques localités du Canton de Vaud. Il habite l'Allemagne, la France et l'Angleterre.

Stipa capillata L. La Stipe chevelue se rencontre sur la colline de 'la Batia, à Sion et aux environs de Brigg; elle a été signalée dans le Canton de Vaud et celui des Grisons. Elle habite l'Allemagne méridionale, le midi de la France, le Piémont, etc.

Stipa pennata L. la stipe plumeuse est plus répandue que la précédente, elle se trouve à St-Maurice, Martigny, Sion, Sierre. etc. On fait avec l'arête plumeuse, teinte de différentes couleurs, des touffes qui servent d'ornement. Elle habite l'Europe centrale et méridionale, l'Espagne, la Grèce, la Sibérie et le nord de l'Afrique.

Kceleria Valesiaca Gaud. (Aira Valesiaca Sut. All. Sesleria variegata Clairv. K. setacea Pers. $\alpha$ glabra Gr. et Godr.) Cette espèce n'est pas rare sur les collines du Bas-Valais, Charrat, Branson, Montorge, SaintLéonard, Sion et on la trouve dans les cantons de Vaud et de Neuchàtel. Elle habite le Tyrol, le Piémont, le centre et le Midi de la France. 
* Aira præcox L. (Avena præcox P. B.) Signalée comme très-rare par Murith à Saint-Léonard et à Sion, où je ne l'ai pas su trouver, elle ne paraît pas se rencontrer ailleurs en Suisse. M. Rapin n'en fait pas mention. Elle habite l'Allemagne du Sud, le Piémont et la plus grande partie de la France. Elle s'est naturalisée aux Etats-Unis.

* Trisetum Gaudinianum Boiss. (Avena Lœfflingiana Gaud. Murith non L. A. Gavanillesii Koch. A. Gaudiniana Rap. Trisetum Cavanillesii Trin. T. IIspanicum Pers.) Cette jolie petite graminée est extrêmement rare; jusqu'à présent on ne l'a trouvée qu'à Montorge, près de Sion, à Saint-Léonard et à Collonge près OutreRhône? Hors du Valais elle n'a été signalée que dans la vallée d'Aoste et à Suze où elle a été déconverte par M. Cesati, d'après un échantillon de l'Herbicr Boissier. Elle avait été indiquée en Dauphiné par Loiseleur, mais il paraît qu'elle n'y existe pas.

M. Boissier a donné à la plante d'Espagne le nom de Trisetum Lœfllingianum Pol. (Avena Lofflingiana L., Cavan. ic., non Gaud. Trisetum Hispanicum Pers). Elle n'habite que les environs de Madrid el d'Aranjuès. La description de Koch se rapporte bien à la plante du Valais, mais la figure de Cavanilles qu'il cite se rapporte à la plante d'Espagne.

* Sclerochloa dura Gaud. P. B. (Cynosurus durus L. Sul. Eleusine dura Lam. Festuca dura Vill. Poa dura Scop.) Celte petile graminée n'est pas rare le long: des chemins sur les collines de Fouly, Saxon, Montorge, Saint-Léonard, etc.; mais on ne la trouve pas ailleurs en Suisse. Elle habite l'Allemagne méridionale, le Piémont et le Midi de la France.

* Poa concinna Gaud. (P. Molinieri $\beta$ D G, fl. fr.) Le 
Pâturin mignon est spécial au Bas-Valais où il croît sur les coteaux de Branson, de Montorge, de Sion, de StLéonard, de Sierre, etc., et dans la vallée de Zermatt près du Glacier de Görner (Reuter). Il n'est signalé hors de la Suisse qu'à Trieste, il manque en Piémont, en France, en Espagne, etc. M. Boissier a découvert en Espagne une plante qu'il avait rapportée d'abord au P. concinna comme var. membranacea, mais ayant reconnu que c'éiait une espèce distincte il l'a décrite sous le nom de Poa ligulata. Notre plante du Valais se distingue du Poa bulbosa florifera par ses panicules plus larges, presque triangulaires, par ses épillets plus grands, plus multiflores, par ses feuilles plus fines. M. Reuter ne l'a jamais vu qu'en Valais, el jamais vivipare. (Note de mon ami Reuter.)

* Poa distans L. (Glyceria dislans Wahl. Greml. ' Ducom.) Cette espèce, rare pour la Suisse, n’a été signalée qu'en Valais, entre Martigny et Sion et aux environs de Viège. On la trouve en France, en Allemagne, en Ang'eterre, surtout sur les côtes maritimes et dans les endroits salés.

* Cynosurus echinatus L. Cette plante se trouve çà et là dans les champs du Bas-Valais et aux environs de Brigg, elle est plutôt adventive que spontanée, car elle ne paraît pas se reproduire d'elle-même. L'espace habite l'Allemagne du Sud, le littoral de l'Adriatique, le Piémont, le Sud-Ouest de la France, le midi de l'Angleterre, l'Afrique du Nord, la Grèce, le Caucase l'Orient (Boissier).

Festuca sciuroides Roth. Rap. Greml (F. bromoides Gaud. non L. Vulpia sciuroides Gm. Ducom.) Elle a été signalée dans le Bas-Valais, près de Vétroz, par Murith, ainsi que dans les cantons de Bâle et de Ge- 
nève. Elle n'est jamais bien abondante chez nous, tandis qu'elle est commune au bord des champs sablonneux de la France, de l'Allemagne et de l'Angleterre.

* Festuca valesiaca Gaud. Ducom. (Festuca ovina var. $\alpha$ Rap. var. $\delta$ Koch). Celte espèce critique est assez répandue dans le Bas-Valais, sur les collines de la Batiâ, des Marques, de Branson, Charrat, Montorge, Saint-Léonard, Sierre, etc. Reichenbach (fl. Germ. exc.) admet l'espèce qui, d'après lui, se trouve en Valais, en Saxe, en Bavière, en Westphalie el en Belgique. C'est par erreur que Moritzi l'indique dans toute la Ghaine des Alpes. Grenier et Godron ne la mentionnent pas dans leur Flore française. Suivant M. Reuter, qui a bien étudié ces diverses formes, la F. valesiaca Gaud., est une bonne espèce qui se reconnaît à ses touffes compactes, toujours bien circonscrites; $\dot{a}$ ses feuilles très-fines, dressées, roides, scabres, quadrangulaires, cendrées-grisâtres ainsi que les tiges et les fleurs; les épillets, rarement colorés, sont ramassés en panicule courte el oblongue, à glumes plus ou moins acuminées ou brièvement aristées. Elle ne croît qu'en Valais, et se trouve sur les terrains secs et sabloneux, sur le bord des champs et sur les petits murs qui les soutiennent. Elle s'élève jusque dans la région alpine, souvent mélangée à la $\mathrm{F}$. duriuscula $\mathrm{L}$. dont elle se distingue, à première vue, par ses feuilles et par sa floraison plus précoce. La F. tenuifolia sibt., qui forme aussi de jolies touffes bien limitées, a la feuilles et les tiges lisses, la panicule étroile, allongée, comme linéaire; les épillets sont un tiers plus petits, ordinairement muliques, souvent colorés en violet. M. Reuter n'est pas bien sûr que nous ayons en Suisse la vérila- 
ble F. ovina L. qu'il a récoltée en Norvège, en Auvergne et dans les Pyrénées.

Triticum Nardus DC. Gaud. (Micropyrum Nardus Rap. Nardurus tenellus Reich. Ducom. Festuca tenuiflora Schrad. Greml. $\gamma$ aristata Koch). Celte jolie Graminée a été signalée par Gaudin à Montorge et à Sion; elle est indiq'?ée aux environs de Genève, et nous l'avons trouvée deux ou trois fois en abondance dans un champ graveleux, près du lac à Versoix. Koch ne signale l'espèce qu'en Istrie et dans le Valais; elle habite surtout les lieux arides du midi et de l'ouest de la France et le Piémont.

Marsilea quadrifolia. L. Cette plante aquatique, signalée par Rapin, dans le marais de Villeneuve, a été indiquée par d'Angreville, à Vouvry et à la Praille. Elle habite les localités marécageuse de l'Allemagne et de la France.

Obs. M. d'Angreville dit que la Pilularia natans, se trouve dans les fossés fangeux du Guerset et de Muzembroz. Est-ce la Pilularia globulifera L. ou la Salvina natans Hoffm. (Marsilea natans L.) qu'il a voulu indiquer? G'est une chose à vérifier.

Lycopodium helveticum L. (Selaginella helvetica Spr.) Celte jolie espèce se trouve sur les rochers près d'outre-Rhône, près du Trient, et vers le village de Gueuroz. Elle habite toute la chaine des Alpes, en Allemagne, en Suisse et dans le Dauphiné; elle paraît manquer en Angleterre. 


\section{ADDITIONS}

Eruca sativa Lam. (Brassica Eruca L.) La Roquette existe à l'état sauvage sur les coteaux du Bas-Valais, Branson, Montorge, St-Léonard, etc., ainsi que dans le district d'Aigle au canton de Vaud. Dans le reste de la Suisse elle est plutôt cultivée comme assaisonnement et s'échappe parfois des jardins. Elle habite l'Allemagne méridionale, le midi de la France, le Piémont, l'Italie, la Grèce, l'Espagne, le nord de l'Afrique et les îles Canaries.

Arabis saxatilis All. (A. nova Vill.) Elle est rare en Valais où je l'ai trouvée au pied du Mlont-0ttan, aux Folateires et sur la colline de St-Léonard. Ponr le reste de la Suisse on ne l'a indiquée que dans quelques localitée du canton de Vaud et sur le Jura près de Soleure. Elle habile le Piémont, la Savoie, le Dauphiné, les Pyrénées et l'Espagne.

A. auriculata Lam. (A. aspera All., A. recta Vill.) Pour le Bas-Valais, elle n'est signalée qu'à Branson oủ j’en ai récolté quelques échantillons. On la retrouve dans les cantons de Soleure, d'Appenzel, de Glaris et des Grisons. Aux environs de Genève, mais hors du canton, elle croit ainsi que la précédente au pied du Salève, dans la vallée du Reposoir et parmi les éboulis du Jura autour du Fort de l'Ecluse; loutes deux sont peu abondantes dans ces diverses localités. L'espèce habite l'Allemagne méridionale, le midi de la France, le Piémont, la Savoie, l'Espagne, l'ltalie et la Sicile.

Draba muralis L. Elle avait été indiquéc, par Mulith et Gaudin, à St-Maurice et au pied du Mont-Ottan où elle n'a pas été retrouvée. Il y a quelques années je l'ai récoltée, avec fes le Dr Dupin, le long des haies 
entre Martigny et Charrat en suivant l'ancienne route; elle élait très-abondante, mais dès lors elle a presque disparu. C'est du reste ce qui arrive souvent aux espèces annuelles et printanières du Valais qui végètent peu ou point quand il y a trop de sécheresse pendant les premiers mois de l'année. Pour le reste de la Suisse, on ne connaît que quelques localités du canton de Bâle. L'espèce habite l'Allemagne, la Hollande, l'Angleterre, l'Ecosse, l'Irlande, la Suède, la France, le Piémont et le Portugal. 
133

\section{TOPOGRAPHIE BOTANIQUE}

J'ai cru devoir donner une énumératiou des plantes les moins communes, classées d'après les localités, comme Gaudin l'a fait dans le VII ${ }^{e}$ volume de sa Flore, dans l'espoir de faciliter les recherches des botanistes qui voudraient faire des herborisations dans le Bas-Valais.

Saint-Gingolph. Si l'on monte jusqu'au creux de Novelle, on trouvera: Rhododendron ferrugineum L., Crocus vernus L., Leucoium vernum L., Gagea lutea, Sch., etc.

Bouveret. Chenopodium rubrum L., G. ficifolium Sm., Sparganium minimum Fries., Typha minima Hopp.

Porte-du-Scex. Arabis sagittata DC., Draba aizoides L., Lunaria rediviva L., Viola mirabilis L., Malva alcea L., Potentilla caulescens L., Sempervivum tectorum L., et var., Cyclamen Neapolitanum Ten. Taxus baccata $L$.

Port-Valais. Trochiscanthes nodiflorus Koch.

Vouvry. Thalictrum flavum L., Dentaria pinnata L., Lunaria rediviva L., Geranium palustre L., Tilia platyphylla Vent,. Opuntia vulgaris Mill., Senecio paludosus L., L., Aster brumalis Nees., Echinospermum Lappula Lehm., Laurus nobilis L., Epipactis palustris L., Herminium monorchis R. Br., Gladiolus palustris Gaud., Sparganium minimum Fries., Acorus Calamus L. En montant jusqu'au petit lac de Tannay, on trouvera beaucoup d'espèces alpines.

Monthey. Ranunculus nemorosus DG., R. philonotis 
Retz., Lithospermum purpureo-cueruleum L., Galanthus nivalis L., Leucoium vernum L, Hemerocallis flava L.?. Gagea lutea Sch., Carex alba L., Scolopendrium officinarum Sw., Lycopodium Helveticum L.

Saint-Maurice. Arabis muralis Bert., A. Turrita I., Cheiranthus Cheiri L., Eruca sativa Lam., Sinapis nigra L., Draba muralis L.?, Cochlearia saxatilis Lam., Senebiera Coronopus Poir., Biscutella laevigata L. et var. saxatilis Schl., Buffonia macrosperma Gay., Ruta graveolens L., Rhamnus alpinus L., Genista ovata W et K.?. Oxytropis pilosa DC., Colutea arborescens L., Trifolium cœspitosum Reyn., Potentilla caulescens L., Sorbus hybrida L., Inula Britannica I., Scorzonera Austriaca L., Lactuca perennis L., Hieracium glaucum All., H. pictum Sch., H, Jacquini Vill., H. amplexicaule L., Seseli bienne Crantz., Trochiscanthes nodiflorus Koch., Veronica prostrata L., Rumex scutatus L., Limodorum abortivum Sw., Spiranthes :estivalis Rich., Iris virescens DC.?, Stipa pennata L., Asplenium Halleri DC.

Bois-Noir. Hepatica triloba DC. fl· albo et fl. roseo, Polygala Chamæbuxus L., Cornus mas L., Saxifraga oppositifolia L., Erica carnea L., Primula variabilis Goup.

Le Rosey, Outre-Rhône, Dorénaz. Ces localités, riches en espèces intéressantes, d'après MM. Rapiu et Muret, doivent être visitéés avec soin. Oxytropis pilosa DG. Pencedanum venetum Koch., Hyssopus officinalis L., Dracocephalum austriacum L. (trèsrare), Potomogeton densus L., Orchis coriophora L.

Mivellaz et la Barme. Viola arenaria DG., Bulbocodium vernum $\mathrm{L}$.

Cascade de Pissevache. Dans les graviers et aux 
environs : Arabis alpina L., A. pumila Wulf.?, Hutchinsia alpina R. Br., Alchemilla alpina L., Miricaria germanica Des., Saxifraga controversa Stern.?, Valeriana tripteris L., Tussilago nivea Vill.?, Senecio viscosus L., Gentiana germanica Willd., Lathræa squamaria L., Erinus alpinus L., Ruscus aculeatus L., Juniperus Sabina L. Contre les rochers: Biscutella Saxatilis Schl., Vesicaria utriculata L, Silene Armeria L., Saxifraga aizoides L., Asplenium Halleri DC.

Vernayaz, Rochers du Trient. Ranunculus philonotis Retz., Primula farinosa L., Pedicularis palustris L., Euphorbîa Gerardiana L., Juniperus Sabina L. Contre les rochers: Primula viscosa Vill., Ceterach officinarum G. B,

En montant à Salvan depuis Vernayaz, on trouve le long du chemin : Circæa intermedia Ehr.. Rosa montana Chaix., Hieracium valesiacum Fries. (Rapin.)

Gueuroz. En montant sur la rive droite du Trient: Arabis muralis Bert., Draba aizoides L., Vesicaria utriculata L., Cochlearia saxatilis Lam., Biscutella lævigata L. et var. saxatilis Schl., Saxifraga exarata Vill. var. leucantha Gaud., Primula viscosa Vill. - Dans les prairies, près du hameau : Corydalis solida Sm. var. australis Hausm., Arenaria trinervia L. Lychnis viscaria L., Saxifraga bulbifera L., Phyteuma betonicæfolium Vill., Orchis sambucina L. Sur les rochers : Silene rupestris L., Scleranthus perennis L., S. verticillatus Reich., Herniaria glabra L., Saxifraga stellaris L. S. cuneifolia L., Veronica fruticulosa L.. Lycopodium Helveticum L.

Entre Gueuroz et Salvan. Potentilla rupestris L., Allosurus crispus Bern.

Martigny. Au pied du mont Ottan : Arabis saxatilis 
L., Draba muralis L.?, Geranium lucidum L., Asplenium septentrionale Sw.

Colline de Bâtia. Anemone Pulsatilla L., var. nutans Gaud., Silene Otites L., Ononis natrix L., Oxytropis campestris DC., 0. pilosa DC., Sempervivum arachnoideum L. var., S. tectorum L. var. Herniaria glabra L., Seseli bienne Crantz., Asperula longiflora W. et K., Hieracium glaucum All, Campanula spicata L., Veronica prostrata L., Euphrasia lutea L., Euphorbia Gerardiana L., Bromus squarrosus L., Phleum asperum L., Kœleria valesiaca Gaud., Stipa capillata L., S. pennata L.

Colline des Marques. Rhus Cotinus L., Ononis Columnæ L., Potentilla recta L., P. inclinata Vill. P. parviflora Gaud., Peucedanum. Venetum Koch., Trinia vulgaris DG., Chrysocoma Linosyris L., Aster Amellus L., Campanula spicata L., C. Bononiensis L., Onosma stellulatum W. et K., Asperula longiflora W. et K., Phelipæa arenaria Walp., Verbascum montanum Schr.. Origanum vulgare L. var. prismaticum Gaud., Hyssopus officinalis L., Festuca valesiaca Gaud., Eragrostis poæoides P. B.

Environs de Martigny : Ranunculus philonotis Retz., Lepidium ruderale L., Sisymbrium Sophia L., Astragalus Onobrychis L., Vicia onobrychioides L., Potentilla verna L. var. cinerea Gaud., Carum bulbocastanum Koch, Pimpinella nigra Koch., Peucedanum Venetum Koch. Chondrilla juncea L., Lactuca viminea Sch., Xeranthemum inapertum Willd., Xanthium strumarium L., Physalis Alkekengi L., Poa piJosa L., P. Eragrostis L., Molinia serotina M. et K.

Entrée de la Vallée de Bagnes: Pyrola arenaria Rap., Hieracium cymosum Fries. 
Branson. Au pied de la colline, contre les rochers; Sempervivum arachnoideum L., S. tectorum L., Orlaya grandiflora L., Marrubium vulgare L.

Dans le village : Calepina Corvini Desv., Anthriscus sylvestris L., Chenopodium opulifolium L., G. rubrum L.

En allant aux Folateires, sur les pelouses, près du village: Helianthemum salicifolium L., Gagea saxatilis Koch.

Au bord des sentiers et des vignes, dans les champs : Adonis flammaea Jacq., Arabis auriculata Lam.. Erysimum Helveticum Gaud., Turritis glabra L., Eruca sativa L., Camelina sylvestris Wallr., Viola tricolor L. var. minima Gaud. Astragalus Onobrychis L., A. Monspessulanus L. Trigonella Monspeliaca L., Vicia onobrychioides L., Lathyrus sphœricus Retz., Potentilla verna L. var. cinerea Gaud., P. recta, L., Scleranthus perennis L., S. verticillatus Reich., Crupina vulgaris Cass., Silybum Marianum Gærtn., Echinops sphaerocephalus L., Lactuca virosa L., L. Scariola L., Tragopogon major Jacq., Podospermum laciniatum DG., Xanthium strumarium L., Myosotis stricta Link. M. collina Reich.,. Veronica verna L., V. præcox L.. V. triphyllos L., Asparagus officinalis $\mathrm{L}$.

Sur les pelouses et sur les rochers: Anemone Pulsatilla L. var. nutans Gaud., Corydalis solida Sm. var. australis Haussm., Saxifraga bulbifera L., Galium pedemontanum L., Achillea tomentosa L., A. setacea Wet.K., A. nobilis L., Artemisia absinthium L., Lappa major Gaertn., Hieracium Peleterianum Mer., Orobanche Artemisiæ Vauch., Phelipaea cœrulea Mey., Hyssopus officinalis L., Thymus serpyllum L. var. pannonicus All., Orchis sambucina L., Bulbocodium vernum L., Carex nitida Host. Tragus racemosus Desf., Scle- 
rochloa dura P. B., Poa concinna Gaud., Festuca valesiaca Gaud., Bromus squarrosus L., Agropyrum glaucum R. et S., Ceterach officinarum C. B.

Au-dessus du sentier, en montant vers Joux-Brulée : Viola mirabilis L., V. sciaphila Koch., Silene Armeria L., Lychnis Coronaria Dess., Galium pedemontanum L.

Dans les taillis, avant les Folateires: Thalictrum pubescens Schl., T. fœtidum L.

Autour des Folateires : Adonis vernalis L., Anemone pulsatilla L. var. nutans Gaud., Arabis muralis Bert. Biscutella laevigata L. et var. saxatilis Schl., Viola arenaria D C., Oxytropis Halleri Bung., Scorzonera Austriaca L.

En descendant vers le Rhône depuis les Folateires: Arabis saxatilis L., Vesicaria utriculata L., Ephedra Helvetica Mey.

Fouly. Le long des sentiers et autour du village on trouve la plupart des espèces de Branson. En outre, dans la forêt au-dessus de Fouly: Vicia sylvatica L. V. dumetorum L. V. pisiformis L. Plus haut: Lychis Flos-Jovis Desr., Geranium Bohemicum L.

De Branson à Martigny : Cerastium arvense L., Tussilago petasites var. hybrida L., Salix fragilis L.

De Martigny à Saxon par la vieille route. Avant Charrat, au bord des haies: Draba muralis L. A Charrat et à Saxon, le long du chemin, sur les collines et sur les rochers: Thalictrum angustifolium L., Adonis vernalis L., Erysimum virgatum 'Koth., Camelina sylvestris Wallr. Alsine fasciculata M. et K., Ononis altissima Lam., Oxytropis Halleri Bung., O. pilosa D C., Astragalus Onobrychis L., A. Monspessulanus L., Vicia Gerardi DC, V. tenuifolia Roth., Sempervivum 
arachnoideum L. var., S. tectorum L., Bryonia alba L., Gnaphalium luteo-album |L., Achillæa setacea W. et K., A. nobilis L., Hieracium lanatum Vill., H. pictum Schl., Tragopogon major Jacq., Scorzonera austriaca Willd., Veronica prostrata L., Chenopodium rubrum L., Thesium intermedium Schr., Populus alba L., Apera interrupta P. B., Kœeleria valesiaca Gúud., Festuca valesiaca Gaud., Bromus squarosus L. Equisetum ramosum Schl.

Riddes : Viola mirabilis L., Astragalus Onobrychis L. Oxytropis pilosa DG., O. Halleri Bung., Centaurea ralesiaca Jord., Hieracium lanatum Vill., Melissa officinalis L., Populus alba L.

Iserabloz au-dessus de Riddes: Sisymbrium Pannonicum Jacq.

Saillon et Leytron : Clematis recta L., Adonis autumnalis L., Glypeola Jonthlaspi L., Ononis Columnae All., Astragalus aristatus L. ?, Amygdalus communis L., Telephium Jmperati L., Lonicera Etrusca Sant., L. Caprifolum L. ?, Turgenia latifolia Hoffm., Artemisia valesiaca Jord., Xeranthemum inapertum Willd., Lactuca saligna L., Hieracium lanatum Vill., Veronica praecox All., Ephedra Helvetica Mey., Acorus calamus L., Selerochloa dura P. B.

Ardon: Buffonia macrosperma Gay., Ononis natrix L., Oxytropis campestris DC., Rosa gallica L., Veronica præcox All., V. acinifolia L., Scilla amœna L. ?

Avent : Clematis recta L., Telephium Imperati L., Chondrilla juncea L., Campanula Bononiensis L., Veronica verna L., Carex gynobasis Vill., Molinia serotina M. et $\mathrm{K}$.

Contey. Adonis autumnalis L., Erysimum Helveticum DG., Ethionema saxatile R. Br.?, Alyssum inca- 
num L. ?, Lepidium graminifolium L., Lonicera Etrusca Sant., Oxytropis pilosa DG., Vicia onobrychioides L., Buplevrum rotundifolium L., Caucalis daucoides L., Turgenia latifolia Hoffm., Rubia tinctorum L. Artemisia valesiaca Jord., Campanula Bononiensis L., Physalis Alkekengi L., Onosma stellulatum W. et K., Veronica acinifolia All. Salvia Sclarea L., Ficus Carica L., Trisetum Gaudinianum Boiss. ?

Vétroz. Clematis recta L., Adonis vernalis L., A. autumnalis L., Myagrum perfoliatum L.?, Neslia paniculata DG., Rosa gallica L. var. pumila Gaud.

Bords de la Morge. Buffonia macrosperma Gay., Astragalus aristatus L'Her., A. monspessulanus L., Herniaria glabra L., Lactuca perennis L., Stachys germanica L.

Etangs de Montorge. Ranunculus trichophyllus Chaix. var. Rioni, R. sceleratus L., Glaucium luteum Scop.?, Utricularia vulgaris $\mathrm{L}$, Orchis laxiflora Lam., Malaxis Lœselii Sw. Typha angustifolia L., Scirpus maritimus L., Carex Pseudo-Gyperus L., Festuca gigantea Vill.

Colline de Montorge. Adonis æstivalis L., A. flammæa Jacq., A. autumnalis L., Isatis tinctoria L. Thlaspi ruderale All., Trigonella monspeliaca L. Rosa montana Ghaix., Buplevrnm rotundifolium L, Asperula longiflora W. et K., Galium Vaillantii DG., Artemisia valesiaca All., Micropus erectus L., Xeranthemum inapertum Willd., Grupina vulgaris Cass., Centaurea valesiaca Jord. G. lanata DC.. Lactuca viminea Sch., Campanula spicata L., Verbascum montanum Schr., Orobanche Artemisiæ Vauch., Phelipæa arenaria Walp., P. cœrulea Mey., Veronica verna L., V. præ$\operatorname{cox}$ All.. V. triphyllos L., Androsace maxima L., 
Éphedra Helvetica Mey., Grocus sativus L., Tragus racemosus Desf., Bromus squarrosus L., Poa concinna Gaud., Sclerochloa dura P. B., Trisetum Gaudinianum Boiss., Triticum Nardus DC., Lolium rigidum Gaud.

Sion. Thalictrum fotidum L., Anemone pulsatilla L. var. nütans Gaud., Adonis æstivalis L., Glaucium corniculatum Curt. Cheiranthus Cheiri L., Sisymbrium Sophia L., Clypeola Jonthlaspi L., Lepidium graminifolium L., Thaspi ruderale All., Isatis tinctoria L., Rhamnus pumilus L., Genista radiata Scop., Gytisus nigricans L.?, Trigonella monspeliaca L., Astragalus Onobrychis L., A. monspessulanus L., Vicia Onobrychioides L., V. lathyroides L., Rosa Eglantiera L.?, Punica Granatum L., Telephium Imperati L., Scleranthus verticillatus Reich., Opuntia vulgaris Mill., Anthriscus cerefolium Hoffm. var. trichosperma Koch., Rubia tinctorum L., Gnaphalium luteo-album L., Achillea nobilis L., Echinops sphærocephalus L., Xeranthemum inapertum Willd., Centaurea valesiaca Jord. Hieracium pictum Schl., Tragopogon major Jacq., Podospernum laciniatum DC, Vinca major L., Onosma stellutatum W. et K., Salvia officinalis L., Rosmarinus officinalis L., Ephedra Helvetica C. A. Mey., Iris Germanica L., I. virescens DC., Bulbocodium vernum L. (Près des mayens de Sion), Tulipa Didieri Jord., Gagea saxatilis Koch. Crocus sativus L. Tragus racemosus Desf., Poa concinna Gaud., Stipa capillata L., S. pennata L., Phleum asperum Vill., Aira præcox L.? Glyceria distans Wahl. (Poa distans L.)?

Saint-Lèonard. Anemone pulsatilla L. var nutans Gaud., Adonis æstivalis L., A. flammæa Jacq., Ranunculus gramineus L., Glaucium corniculatum Curt., Arabis saxatilis All., Turritis glabra L., Viola tricolor 
L. var. minima Gaud., Ononis Columnæ All., Onobrychis arenaria DG., Amygdalus communis L., Potentilla verna L. var. cinerea Gaud., Scleranthus verticillatus Reich., Buplevrum rotundifolium L., Galiım Vaillantii DG., Achillæa nobilis L., Crupina vulgaris Cass., Xeranthemum inapertum Willd. Centaurea valesiaca Jord., Veronica verna L., V. præcox All., V. acinifolia L., Androsace maxima L., Bulbocodium vernum L. (fl. alb.), Carex humilis Leys., Poa concinna Gaud., Sclerochloa dura P. B., Cynosurus echinatus L., Festuca Valesiaca Gaud., Koleria Valesiaca Gaud., Trisetum Gaudinianum Bois.

Plâtrières entre Saint-Léonard et Sierre. Ranunculus gramineus L., Erysimum Helveticum DC., Buffonia macrosperma Gay., Colutea arborescens L., Ononis columnae All., Telephium Imperati L., Echinops sphærocephalus L., Xeranthemum inapertum Willd., Artemisia valesiaca All., Lactuca viminea Sch. Koch., Euphrasia lutea L., Onosma stellulatum W. et K., Phelipæa cœrulea Mey., Hyssopus officinalis L, Thymus serpillum L. var. pannonicus All., Asparagus officinalis L., Stipa capillata L., S. pennata L., Molinia serotina M. et K.

Sierre et bois de Finges : Adonis autumnalis L., Erysimum Helvéticum DC., Eruca sativa L., Buffonia macrosperma Gay., Ruta graveolens L., Astragalus Onobrychis L., A. Monspessulanus L., Oxytropis pilosa DG., O. Halleri Bung., Onobrychis arenaria DG., Colutea arborescens L., Medicago falcata L. var. minor. Gaud., Ononis Columnæ All., Telephium Imperati L., Pimpinella nigra Koch., Buplevrum rotundifolium L., Rubia tinctorum L., Scabiosa columbaria L. var. tenuisecta Gaud., Chrysocoma Linosyris L., Aster Amellus L., Gnaphalium luteo-album L., Filago 
Gallica L., Micropus erectus L., Artemisia absinthium L., A. valesiaca All., Achillaea tomentosa L., A. setacea W. et K., A. nobilis L., Crupina vulgaris Cass., Xeranthemum inapertum Willd., Centaurea valesiaca Jord., Chondrilla juncea L., Lactuca viminea Sch. Koch., Hieracium praealtum Vill., H. pictum Schl., Pyrola Chlorantha Sw. (Forêt de Finges), Onosma stellulatum W. et K., Veronica acinifolia L., V. verna L. V. praecox All. Euphrasia lutea L. E. viscosa L. (Forêt de Finges), Androsace maxima L., Euphorbia Gerardiana L., E. segetalis L. var., Chenopodium Botrys L. (For. de Finges) Populus alba L., Tragus racemosus Desf., Poa eragrostis L.

Varonne: Buffonia macrosperma Gay., Helianthemum fumana L., Coronilla coronata Gaud. (G. minima L.), Potentilla caulescens L., Rubia tinctorum L Lactuca virosa L., L. Scariola L., L. Augustana All., Crepis fœetida L., Hieracium valesiacum Fr., H. lanatum Vill. (entre Varonne et Inden), Euphrasia riscosa L., E. lutea L., Phœlipea cœrulea Mey., Origanum vulgare L. var. prismaticum Gaud., Calamintha nepetoides Jord. (C. nepeta Clairv. Gaud.), Blitum virgatum L. 


\section{NOTICE SUR G. REUTER}

Pendant que s'imprimaient ces dernières pages, j’ai eu le chagrin de perdre mon vieux et excellent ami G. Reuter, directeur du Jardin botanique de Genève. C'était le principal chef de celle bande du Valais qui a déjà vu partir le $D^{r}$ Mercier, le curé Chavin, le Dr Dupin et dont j'ai raconté quelques-unes des excursions.

Notre ami Reuter avait abandonné la gravure, entraîné qu'il était par son goùt pour l'élude des fleurs. Apprécié et encouragé par le pasteur Vaucher qui le rencontrait souvent au Jardin des plantes, il fut présenté par lui au professeur De Candolle qui se l'attacha comme conservateur de son herbier. Plus tard il fut appelé à remplir le même poste chez M. Ed. Boissier dont il devint le compagnon de travail et l'ami dévoué pendant un grand nombre d'années. C'est par ses propres efforts et par un travail incessant que Reuter avait acquis une profonde érudition en botanique, science à laquelle il a rendu de véritables services, grâce à son coup d'œil remarquable et à sa prodigieuse mémoire. Il a travailié à la Flore de Zante, il a écrit la monographie des Orobanches pour le Prodrome, il a coopéré à plusieurs mémoires publiés par M. Boissier, enfin il a fait connaître à fond la Flore des environs de Genève au moyen de son Catalogue raisonné. Nommé directeur du Jardin bolanique, il a su continuer les traditions de l'illustre fondateur et il a créé ces montagnes artificielles, couvertes de plantes alpines rares et variées que les botanistes et lles amateurs viennent admirer. Les derniers temps de sa vie onl été em- 


\section{3}

ployés à classer el à déterminer le grand herbier Dulessert avec M. le Dr Nuller, M. Rapin, et les autres inembres de la Commission.

Homme instruit et modeste - trop modeste mème - ne cherchant jamais à se laire valoir, rendant de nombreux services avec la plus rare bienveillance, essentiellement consciencieux et esclave du devoir, cœur simple, bon et sincèrement chrétien, tel était celui que nous regrettons et dont la mort foudroyante et prématurée sera vivement sentie par ses nombreux amis et par tous les botanistes qui avaient si souvent recours à sa complaisance et à son savoir. 



\section{TABLE DES MATIĖRES}

Pages.

Introduclion. . . . . . . . . . . . 3

Herborisations. . . . . . . . . . . . 7

Catalogue........ . . . . . . 47

Noles. . . . . . . . . . . . . 89

Additions . . . . . . . . . . . 131

Topographie botanique . . . . . . . . 139

Notice sur G. Reuter . . . . . . . . . 1/1 

= 


\section{Chez $\mathbb{M} . \mathrm{G} \cap \mathbb{R} \mathrm{G}$, Libraire-Editeur}

\section{A GENÈVE \& BALE}

Dp" Ch. Fanconnet. Herborisations it Salève, in-8. 1867 Fr. 1 - Promenades botanirgues aux Voirons et supplément aux herborisations à Salève, in-8. 1868 . . .

Boissiea. (Edm.) Voyage hotanicfue dans le nirli de l'Espaone, pendant l'année 1837 ; 2 vol. or. in-4, avee $206 \mathrm{pl}$. colorées. Prix réduit, au lieu de fr 400 . Græcir et AEgypto in Indire fines.

Vol. I. Thalamiflorie.

Vol. II. Caicrilore polypetalic sous presse).

- Icones Euphorbiarmin ou figur s le 122 espices du genre

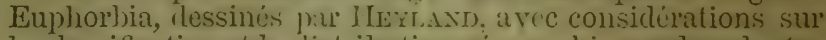
la classification et. la ilistribution géographiques des plantes de ce genre, in-fol. avcc $1.20 \mathrm{pl}$.

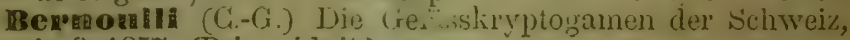
in-8. 1857. (Prix réduit.)

Candolle (Alph. de) Tui de in Nomenclature hotaniogue, adoptées par le cungris intes en août $1867,2^{\text {me }}$ édition. In -

- Etudes historiques et plinil particulier sur les Sciences ni inal de botanique it Paris 37

iques sur les sciences, en elles. (Sous presse.)

Christ (H.) Ueler dio Pllimzendecle des Jurageloirgs. In-8, 1868 .

Hagevbach $(C-F$. $)$ Tentamen loric basileensis exhib. plantas phanerogamas sponte nascent. C. Bauhini effig. et 2 taj. col.Acced. Supplementum. In-8. 1821-13 (publiè à 17-50). Prix réduit

Theller (Jean). Monographie de la famille des Résédacés. In-4, 239 pages de texte avec 10 planches lithographiées. 1857 (publié à $25 \mathrm{fr}$.)

- Principes de classilicatiun des Lichens et énumération des Lichens des environs '? Gerève. In-1 avec 3 planches 1862 .

Tentea (G.-F) Catalorue , es riantes vasculaires des envi-

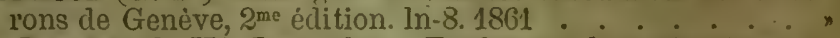

Selnache (D.-H.) Les arbure Etudes sur leur structure et leur végétation. Traduit de -ailemand par E. MIorrex. Ouvracre publié sous les ausiries de feu M. le baron Alex. DE Huniold 1 vol. in-8, iliustré de 205 gravures sur Joois et $5 \mathrm{pl}$. lith, au lieu de fi. 17

Tiss iene (Chanoine du Gr ed St-Bernard). Guide du botaniste sur le Grand St-Berr.urd. 1868. 



\section{OK313 F38 York Botanical Garden Library \\ Fauconnet, Charles/Herborisations a Sale \\ gen

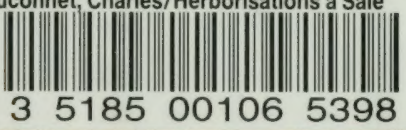

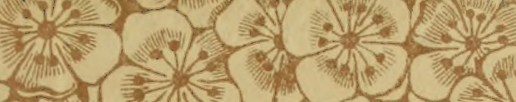

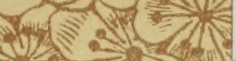

...iil) 1 (I) (1) iin)

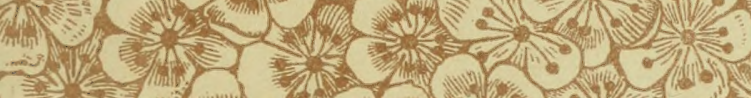

(a) $2 \sqrt{2}$

\section{3.} end

T

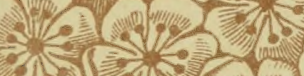

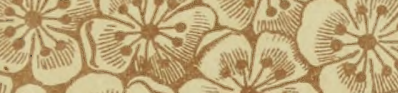

$\div$

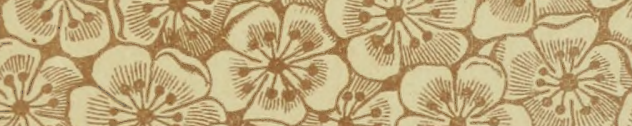
$f_{1}$

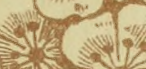

(1)

S1M

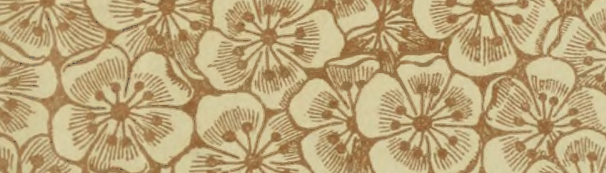

(i) 
